\title{
Vegetable induced gene expression changes in anticarcinogenic pathways in human and mouse tissues
}

Citation for published version (APA):

van Breda, S. G. J. (2004). Vegetable induced gene expression changes in anticarcinogenic pathways in human and mouse tissues. [Doctoral Thesis, Maastricht University]. Maastricht University. https://doi.org/10.26481/dis.20041222sb

Document status and date:

Published: 01/01/2004

DOI:

10.26481/dis.20041222sb

Document Version:

Publisher's PDF, also known as Version of record

\section{Please check the document version of this publication:}

- A submitted manuscript is the version of the article upon submission and before peer-review. There can be important differences between the submitted version and the official published version of record.

People interested in the research are advised to contact the author for the final version of the publication, or visit the DOI to the publisher's website.

- The final author version and the galley proof are versions of the publication after peer review.

- The final published version features the final layout of the paper including the volume, issue and page numbers.

Link to publication

\footnotetext{
General rights rights.

- You may freely distribute the URL identifying the publication in the public portal. please follow below link for the End User Agreement:

www.umlib.nl/taverne-license

Take down policy

If you believe that this document breaches copyright please contact us at:

repository@maastrichtuniversity.nl

providing details and we will investigate your claim.
}

Copyright and moral rights for the publications made accessible in the public portal are retained by the authors and/or other copyright owners and it is a condition of accessing publications that users recognise and abide by the legal requirements associated with these

- Users may download and print one copy of any publication from the public portal for the purpose of private study or research.

- You may not further distribute the material or use it for any profit-making activity or commercial gain

If the publication is distributed under the terms of Article $25 \mathrm{fa}$ of the Dutch Copyright Act, indicated by the "Taverne" license above, 
Vegetable induced gene expression changes in anticarcinogenic pathways in human and mouse tissues 
(1) S.G.J. van Breda, Maastricht 2004

ISBN: 90-6464-925-1

Lay-out:

Cover design:

Production:
Simone van Breda

Simone van Breda/ Sumero van Agen

Grafisch bedrijf Ponsen \& Looijen B.V.

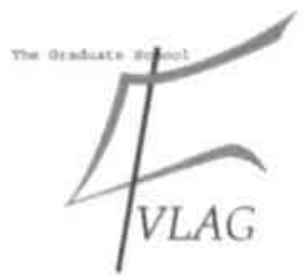

The studies presented in this thesis were performed at the Nutrition and Toxicology Research Institute Maastricht (NUTRIM) which participates in the Graduate School VLAG (Food Technology, Agrobiotechnology, Nutrition and Health Sciences), accredited by the Royal Netherlands Academy of Arts and Sciences. 


\section{Vegetable induced gene expression changes in anticarcinogenic pathways in human and mouse tissues}

\section{PROEFSCHRIFT}

ter verkrijging van de graad van doctor aan de Universiteit Maastricht, op gezag van de Rector Magnificus, Prof. mr. G.P.M.F. Mols volgens het besluit van het

College van Decanen, in het openbaar te verdedigen op woensdag 22 december 2004 om 14.00 uur

door

Simone Gerty Jeannette van Breda

geboren te Hulsberg op 2 januari 1977 


\section{Promotor:}

Prof.dr. J.C.S. Kleinjans

\section{Co-promotor:}

Dr. J.H.M. van Delft

\section{Beoordelingscommissie:}

Prof.dr. E.C.M. Mariman (voorzitter)

Prof.dr. R-j. Brummer

Prof.dr. M.J.A.P. Daemen

Prof.dr. M. Müller (Wageningen Universiteit)

Dr. H.J.M. Smeets

Financial support by the Dr. Ir. van de Laar Stichting, and Corning Life Sciences B.V, for the publication of this thesis is gratefully acknowledged. 




\section{Contents}

Chapter 1

Chapter 2

Chapter 3

Chapter 4

Chapter 5

Chapter 6

Chapter 7
General introduction

Altered vegetable intake affects pivotal carcinogenesis pathways in colon mucosa from adenoma patients and controls

Dose dependent effect of a vegetable mixture on 73 gene expression in colon mucosa of C57BL6 female mice

Identification of proteins differentially expressed in 101 colon mucosa of C57BL.6 female mice by altered vegetable consumption

Gene expression modulation by specific vegetables 121 in colon mucosa of C57BL6 female mice

Gene expression modulation in lung cancer 145 preventive pathways by vegetables in female C57BL6 mice

Summary and general discussion 169

Nederlandse samenvatting 181

Dankwoord 189

Curriculum vitae

List of publications

Abbreviations

196 



\section{Chapter 1}

\section{General introduction}




\section{Vegetables and Cancer Prevention}

\section{$1.1 \quad$ Vegetables}

Historically, consumption of particular vegetables was thought to prevent or cure ailments ranging from headaches to heart disease. In fact, early medicine revolved largely around the prescription of specific plant food concoctions for certain disorders. The diverse medicinal properties of extracts, juices, or poultices obtained from plants have led to the cultivation of these in many parts of the world. ${ }^{1-3}$ Botanically, a vegetable is any part of a plant not involved in the sexual reproduction of the plant. Vegetables are typically the cultivated or gathered leaves, roots, stalks, bulbs and flowers of plants. Some foods that are culinary vegetables are botanically classified as fruits; these include avocados, cucumbers, aubergines, peppers, tomatoes, pumpkins and courgettes. Vegetables include artichokes, asparagus, beets, broccoli, Brussels sprouts, cabbage, carrots, cauliflower, chard, endive, fennel, garlic, kohlrabi, leeks, lettuce, mushrooms, okra, onions, parsley, parsnips, radishes, rhubarb, spinach, turnips, legumes and various green leafy vegetables. Vegetables may be grouped into categories that are either botanically or culinary meaningful, for example: 'green leafy' (spinach, lettuce), 'cruciferous' (broccoli, cauliflower, kohlrabi, Brussels sprouts, cabbage), 'allium' (onions, garlic, scallions, chives and leeks), 'raw' (uncooked vegetables), 'orange' (carrots, pumpkin, winter squash), and legumes (peas, beans).1.4.5

\subsection{Epidemiological Evidence of Cancer Prevention by Vegetables}

As early as 1933, a case-control study by Stocks and Karn in the UK suggested an inverse association between intakes of certain vegetables against all cancer sites ${ }^{6}$ Since that time, over 250 epidemiological studies (case-control, cohort or ecological correlations) have been conducted worldwide to investigate the relationship between vegetable and fruit consumption and cancer risk. In most of these studies, fruit and vegetables are considered collectively, although different types of vegetables and fruits have been investigated separately. This literature on vegetables and fruit consumption and the prevention of cancer has been reviewed in several scientific papers. ${ }^{1,3,7,8}$ Prevention of stomach cancer has been the most studied, followed by cancers of the colon, esophagus, lung, oral cavity and pharynx, rectum and breast; more than ten studies have focused on each of these sites. About $80 \%$ of these studies found a significant protective effect of overall consumption of vegetables and/or fruit, or at least of some types of vegetables and fruits for cancers of the following targets: stomach, esophagus, lung, oral cavity and pharynx, endometrium, pancreas, colon, and skin. Prostate cancer is the only cancer for which the majority of 
studies have not reported at least one statistically significant inverse association. On the whole, evidence that vegetables are protective is stronger than for fruits, but this may simply reflect the generally higher consumption of vegetables worldwide. 1, 3,7, 8

In 1997, the World Cancer Research Fund and the American Institute for Cancer Research (WCRF/AICR) jointly published an extensive, global review of the role of food and nutrition in the prevention of cancer. Vegetables and fruits received considerable attention in this review. Based on statistical and other considerations, the WCRF/AICR panel ranked the evidence that a high intake of vegetables and fruits decreased the risk of cancer as shown in Table 1. Consumption of higher levels of vegetables and fruits is associated consistently with a reduced risk of cancer at most sites. The association is most marked for epithelial cancers-particularly those of the alimentary and respiratory tracts-and, currently, is weak to nonexistent for hormone-related cancers. ${ }^{4}$ Diets containing substantial amounts (>400 g/day) of a variety of vegetables and fruits may reduce the overall incidence of cancer by over 20 percent. ${ }^{10}$

\section{Table 1. Ranking of cancer risk reduction at various sites from vegetables and fruits."}

\begin{tabular}{|llll|}
\hline & Convincing & Probable & Possible \\
\hline Vegetables and & Oral cavity & Larynx & Ovary \\
fruits combined & Pharynx & Pancreas & Cervix \\
& Esophagus & Breast & Endometrium \\
& Stomach & Bladder & thyroid \\
& Lung & & \\
\hline Vegetables only & Colon & & Liver \\
& Rectum & & Prostate \\
& & Kidney \\
\hline
\end{tabular}

Next to this assessment of cancer risk reduction, an evaluation can be made of which specific vegetables and fruits are responsible for the beneficial effects. Consumption of vegetables in general and raw vegetables in particular has most often been found to be inversely related with the risk of any type of cancer. More than 85 percent of studies which investigated raw vegetables reported a protective association. Data for allium vegetables, carrots, green vegetables, cruciferous vegetables, and tomatoes are also fairly consistent: for each of these types of vegetables, at least 70 percent of studies showed a protective association. Table 2 summarizes the evidence about the most protective vegetable types. ${ }^{1,4}$

Studies published since the WCRF/AICR review confirm and extend the recommendations for vegetables and fruit. ${ }^{11},{ }^{12}$ Most recently, The World Health Organization and the Food and Agriculture Organization of the United Nations have released a report of their Expert Consultation on Diet, Nutrition and the Prevention of Chronic Diseases which suggested that a high intake of vegetables and fruit probably reduces the risk of cancers of the oral cavity, esophagus, stomach and colorectum. ${ }^{13}$ 
Table 2. Evidence relating particular types of vegetables and fruits to cancer risk reduction."

\begin{tabular}{|c|c|c|c|}
\hline & Convincing & Probable & Possible \\
\hline Vegetables in general & $\begin{array}{l}\text { Stomach } \\
\text { Colon } \\
\text { Esophagus } \\
\text { Lang } \\
\text { Oral cavity and pharyns } \\
\text { Rectum }\end{array}$ & $\begin{array}{l}\text { Larynx } \\
\text { Pancreas } \\
\text { Breast } \\
\text { Bladder }\end{array}$ & $\begin{array}{l}\text { Ovary } \\
\text { Endometriurn } \\
\text { Cervix } \\
\text { Liver } \\
\text { Prostate } \\
\text { Kidncy } \\
\text { Thyroid }\end{array}$ \\
\hline Allium vegetables & Stomach & $\begin{array}{l}\text { Colon } \\
\text { Recrum }\end{array}$ & \\
\hline Tomatoes & $\begin{array}{l}\text { Stomach } \\
\text { Esophaggus } \\
\text { Lung }\end{array}$ & $\begin{array}{l}\text { Colon } \\
\text { Oral cavity and pharynx } \\
\text { Rectum }\end{array}$ & \\
\hline Green (leafy) vegetables & $\begin{array}{l}\text { Stomach } \\
\text { Colon } \\
\text { Esophagus } \\
\text { Lang } \\
\text { Oral cavity and pharynx }\end{array}$ & $\begin{array}{l}\text { Breast } \\
\text { Bladder }\end{array}$ & \\
\hline Legumes & Stomach & & \\
\hline Raw vegetables & $\begin{array}{l}\text { Stomach } \\
\text { Esophagus }\end{array}$ & $\begin{array}{l}\text { Colon } \\
\text { Oral cavity and pharynx } \\
\text { Pancreas }\end{array}$ & \\
\hline Carrots & $\begin{array}{l}\text { Stomach } \\
\text { Lung } \\
\text { Oral cavity and pharynx } \\
\text { Rectum }\end{array}$ & $\begin{array}{l}\text { Breast } \\
\text { Bladder } \\
\text { Colon }\end{array}$ & \\
\hline Cruciferous & $\begin{array}{l}\text { Rectum } \\
\text { Colon }\end{array}$ & Thyroid & $\begin{array}{l}\text { Oral cavity and } \\
\text { pharynx }\end{array}$ \\
\hline Fruits in general & $\begin{array}{l}\text { Stomach } \\
\text { Lung } \\
\text { Oral cavity and pharynx } \\
\text { Breast } \\
\text { Pancreas }\end{array}$ & $\begin{array}{l}\text { Colon } \\
\text { Esophagus } \\
\text { Rectum }\end{array}$ & Bladder \\
\hline Citrus fruits & $\begin{array}{l}\text { Stomach } \\
\text { Esophagus } \\
\text { Oral cavity and pharynx }\end{array}$ & Rectum & $\begin{array}{l}\text { Breast } \\
\text { Pancreas }\end{array}$ \\
\hline
\end{tabular}


However, it is stated that the support for a broad and strong protective effect of higher vegetable and fruit intake has been weakened by the results from recent prospective studies ${ }^{14} 19$, which have not supported important protective effects for cancers of the lung and breast, and have suggested that the reduction in colorectal cancer risk may be modest. These discordant results, which add to concerns about the potential for bias in casecontrol studies, also suggests the need for some caution regarding conclusions about intake of fruits and vegetables and the risk of oral, esophageal and stomach cancers, which have not been adequately examined in lange prospective studies. Although support for a broad and strong protective effect of higher fruit and vegetable intake against cancer incidence has been weakened, modest benefits of increasing fruit and vegetable intake have not been excluded and probably do exist. Further research is needed without epidemiological bias using sensitive biomarkers to strengthen the findings. Investigations at the molecular and submolecular levels may identify the genetic and biochemical mechanisms by which vegetables affect cancer risk.

\subsection{Experimental Evidence of Cancer Prevention by Vegetables: Animal Studies}

Laboratory animal models are used to test the effects of vegetables on cancer. The two species most often used to investigate the effects of vegetables and vegetable components on different stages of the carcinogenic process are the mouse and rat. In particular, the C57BL6 mouse is used, because of the possibility of using transgenic and knockout variants. ${ }^{2023}$ Most of the studies are conducted in which cancer has been experimentally induced (usually via a chemical carcinogen or irradiation) in animals fed specified amounts of certain vegetables or vegetable components. ${ }^{25.35}$ In most studies, it was found that animals fed vegetables or vegetable constituents experienced fewer tumours $23,34,36$, fewer metastases ${ }^{36}$, less DNA damage ${ }^{37}$, higher levels of enzymes involved in the detoxification of carcinogens $28,32,37.39$, or other indicatives of a lower cancer risk, ${ }^{24,27,40,41}$ However, to obtain statistically significant results, carcinogens and nutrients are both commonly administered to animals in doses far higher than in any conceivable real-life equivalent. The extrapolation of results from animal studies to humans is further hampered by species differences $^{22}$, the use of carcinogens, and the use of genetically susceptible animals. Main advantages of animal studies are that laboratory conditions can be accurately controlled in ways impossible in any human environment, and that genetic variability between individuals can largely be avoided. Many insights into anticarcinogenic mechanism have been gained by these means. However, the genetic pathways through which vegetables exert their effect are still mostly unknown, because few studies have investigated the effect of vegetables and vegetable constituents on gene expression changes in target organs. In 
section 2.2.4 and 2.3.4, this topic will be described in more detail for two target organs, i.e. the colon and rectum and the lung.

\subsection{Mechanisms of Anticarcinogenesis}

Many potentially mechanisms have been proposed to account for protection against cancer by vegetables. Numerous constituents found in vegetables, including micronutrients (nutritive compounds, e.g. carotenoids, vitamin $\mathrm{C}$ and $\mathrm{E}$, folic acid, selenium), dietary fiber and phytochemicals (non-nutritive bioactive compounds with no known nutritional value, e.g. flavonoids, indoles, isothioscyanates, glucosinulates), and interactions among these constituents might contribute to the ability of these foods to reduce cancer risk. Evidence for the cancer-preventing actions of these agents mainly comes from animal studies and in vitm studies, which have been shown that they are capable of preventing cancer either directly or via complex interactions with the body's metabolic and molecular processes. ${ }^{4,43}$. ${ }^{45} \mathrm{It}$ is thought that the potent anticarcinogenic agents act in concert to prevent cancer ('teamwork'), rather than that a single substance is responsible for the protective effect ('magic bullet'). ${ }^{46}$

Because cancer is a multistep process occurring over an extended time frame, there is a number of possible stages at which the process could be halted, inhibited, delayed or even reversed. ${ }^{47}$ The multiple phases involved in the pathogenesis of cancer offer the basis of most classifications of anticarcinogenic mechanisms of agents present in vegetables. ${ }^{48-51}$ Some mechanisms are reiterated several times in different phases of the process, while others are strictly interconnected or partially overlapping. Furthermore, the agents, which are possibly responsible, have both complementary and overlapping mechanisms of action. Table 3 gives an overview of a selection of proposed anticarcinogenic mechanisms, together with examples of protective agents, their specific mode of action and their vegetable source. Here, the mechanisms are divided into two categories; i.e., I) blocking mechanisms (inhibition of mutation and cancer initiation), and II) suppressing mechanisms (suppression of promotion, progression, invasion, and metastasis) (classification taken from Wattenberg (1992) ${ }^{52}$, supplemented with information from De Flora $(1998)^{49}$, Kellof et al $(1999)^{50}$ ). In most of the presented mechanisms, modulation of gene expression plays a role, however, the molecular targets at the genome level and the involved genetic pathways are mostly unknown. 
Tabel 3. Anticarcinogenic mechanisms of agents present in vegetables.

\begin{tabular}{|c|c|c|c|c|}
\hline Process of anticarcinogenesis & Protective agent & Mode of action & Vegetable source & Ref, \\
\hline \multicolumn{5}{|l|}{ 1) Blocking mechanisms } \\
\hline Prevention of carcinogen uptake & Dietany fiber & Adsorbation of carcinogens & All vegetabiles & 33 \\
\hline \multirow{2}{*}{$\begin{array}{l}\text { Inhibition of non-enzymatic } \\
\text { endogenous carcinogen formation }\end{array}$} & Vitamin C & Scavenging of nitrite to prevent formation of nitrosamines & All vegetables & 34 \\
\hline & Flavonoids & Inhibition of the conversion of nitrate to nitrite & Allium vegetables & s \\
\hline \multirow{3}{*}{$\begin{array}{l}\text { Inhibition of enzymatic carcinogen } \\
\text { formation/activation: modulation } \\
\text { of biotransformation enzymes }\end{array}$} & $\begin{array}{l}\text { Isothiocyanates, } \\
\text { indoles }\end{array}$ & $\begin{array}{l}\text { Inhibition of activation by phase-I enzymes; Induction of } \\
\text { phase-I detoxification and phase-II conjugation pathways }\end{array}$ & Cruciferous vegetables & 4. 38 \\
\hline & Flavonoids & Inhibition of activation by phase-I enzymes & Allium vegetables & 51,5 \\
\hline & $\begin{array}{l}\text { Onganosulfur } \\
\text { compounds }\end{array}$ & Inhibition of phase-1 and induction of phase $-1 /$ enzymes & Allium vegetables & $s$ \\
\hline \multirow[t]{5}{*}{$\begin{array}{l}\text { Blocking, quenching or scavenging } \\
\text { of reactive metabolites }\end{array}$} & Flavonoids & $\begin{array}{l}\text { Direct scavenging of reactive oxygen species and free } \\
\text { radicals; inhibition of xanchine oxidase activity }\end{array}$ & Alfium vegetables: & 58,5 \\
\hline & Isothiocyanates & Scavenging of free radicals & Cruciferous vegetables & $m, 6 !$ \\
\hline & Vitamin $C$ & Scavenging of free radicals; Quenching of singlet oxygen & All vegetables & ke $\alpha$ \\
\hline & Carotenoids & $\begin{array}{l}\text { Quenching of singlet oxygen; Protection of DNA } \\
\text { nucleophilic sites }\end{array}$ & $\begin{array}{l}\text { Orange and Green } \\
\text { leafy vegetables }\end{array}$ & 63.65 \\
\hline & Vitamin E & $\begin{array}{l}\text { Intracellular antioxidane protection of cell membranes } \\
\text { from lipid peroxidation by trapping peroxyl radicals }\end{array}$ & Green vegetables & $\omega$ \\
\hline \multirow[t]{2}{*}{$\begin{array}{l}\text { Induction of DNA repair and - } \\
\text { synthexis }\end{array}$} & Protease inhibitors & $\begin{array}{l}\text { Inducing DNA repair by enhancing poly(ADP. } \\
\text { ribosyl) transferase }\end{array}$ & Legumes & 67 \\
\hline & Flavonoids & Inhibition of DNA topoisomerase II & Allium vegetables & (a) \\
\hline \multicolumn{5}{|l|}{ II) Suppressing mechanisms } \\
\hline \multirow[t]{6}{*}{$\begin{array}{l}\text { Inhibition of cell proliferation and } \\
\text { replication (cell cycle arrest) }\end{array}$} & Quercetin & Blocking of G1/S transition of the cell cycle & Allium vegetables & sio \\
\hline & Protease inhibitors & Repression of the NF-KB signaling pathway & Legumes & so \\
\hline & $\beta$-carotene & $\begin{array}{l}\text { Inhibition of polyamine metabolism by downregulating } \\
\text { omithine decarboxylase }\end{array}$ & $\begin{array}{l}\text { Orange and Green } \\
\text { leafy vegetabies }\end{array}$ & $n+3$ \\
\hline & Dietary fiber & Inhibirion of secondary bile acids & All vogetables & 31 \\
\hline & Indoles & Inhibition of cyclin-dependent kanase 6 & Cruciferous vegetables & 3 \\
\hline & Flavone & Downregulation of Cyclin B and $E_{2}$ induction of $p^{21}$ & Alliurn vegetabies & 73 \\
\hline
\end{tabular}


Table 3. (Continued) Anticarcinogenic mechanisms of agents present in vegetables.

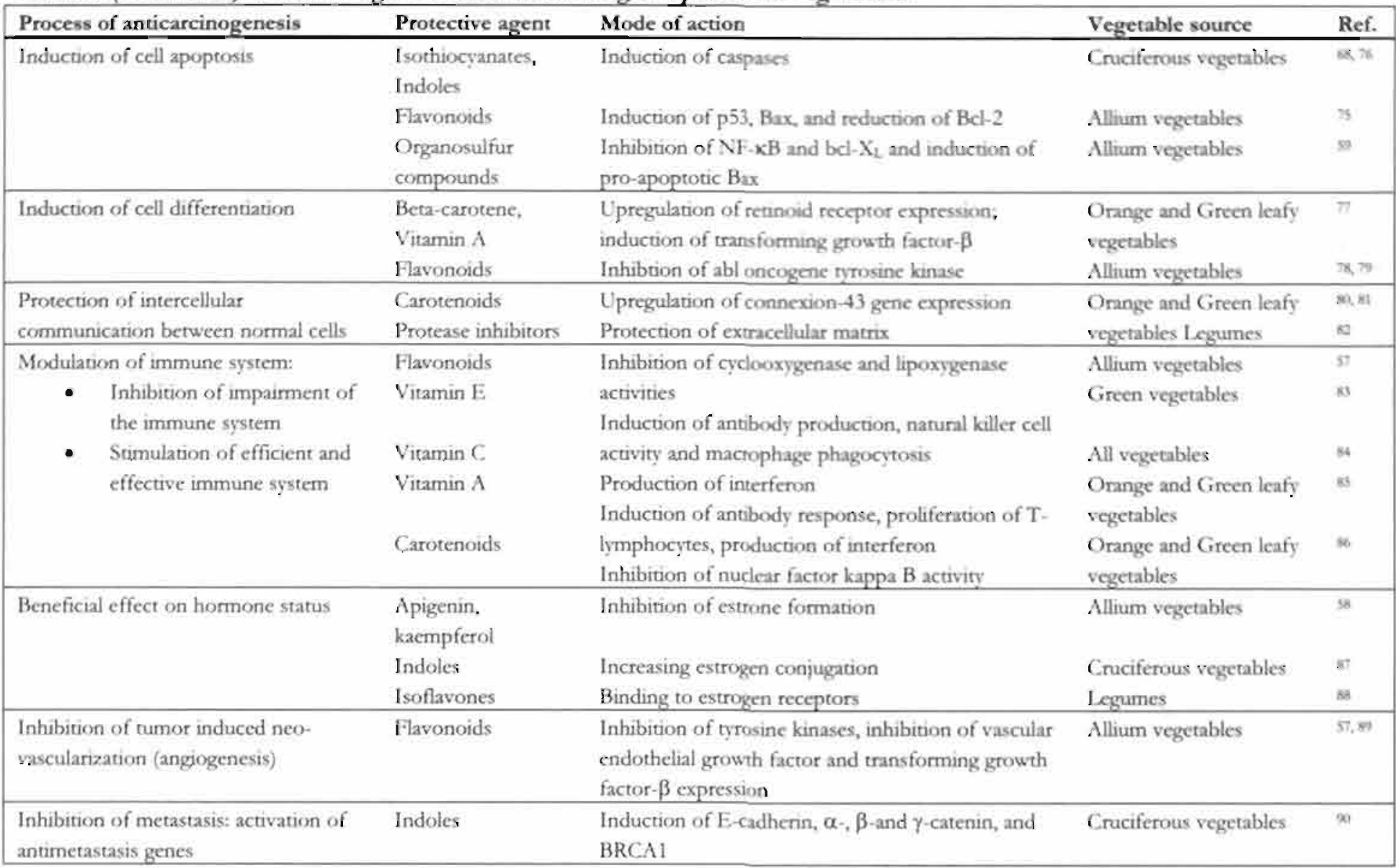




\section{Target Organs}

\subsection{Introduction}

The epidemiological proof for cancer prevention by vegetables is most evident for cancers of the alimentary and respiratory tract. The colon and rectum, and the lungs are of particular interest in these studies. The high incidence of both types of cancer (see sections 2.2 and 2.3), together with the epidemiological rationale of prevention of these cancers by vegetables, makes these two types of cancer highly convenient for elucidating the mechanisms of cancer prevention by vegetables. Furthermore, these two organ systems differ in respect to the part/components of the vegetables that reaches the tissues. In contrary of the lungs, the colon and rectum are both sides of contact, i.e., as the vegetables pass through the colon and rectum, they come into close contact with the intestinal lining. The substances that reach the lung (and also the colon and rectum), have been modulated by the first-pass metabolism: substances that are absorbed via intestinal blood capillaries will first have to pass the liver before they reach the systemic circulation. Here, many substances are extensively metabolized.

In this paragraph, the tissue characteristics (histology), the epidemiology and etiology, the genes and involved pathways in cancer development, and a summary of the knowledge of modulation of cancer risk by vegetables for the colon and rectum, and lungs, are described.

\subsection{The Colon and Rectum}

\subsubsection{Histology of the Human Colon and Rectum}

The large intestine is part of the alimentary tract, and is subdivided into the cecum, colon (ascending, transverse, descending and sigmoid), rectum, and anus. It has three functions: 1) concentration of fecal effluent through water and electrolyte absorption; 2) storage and controlled elimination of feces; and 3) digestion and absorption of undigested food (complex sugars). The colon accounts for almost the entire length of the large intestine $(125 \mathrm{~cm})$. Its wall is composed of four broad layers: the mucosa, submucosa, muscularis externa, and serosa (Figure 1). The lumen is lined by an epithelium, deep to which is a loose connective tissue known as the lamina propria. Surrounding this connective tissue coat is the muscularis mucosae, composed of an inner circular layer and an outer longitudinal layer of smooth muscle. The epithelium, lamina propria, and muscularis mucosae are collectively called the mucosa. The epithelium is richly endowed with crypts of Lieberkühn which consists of surface absorptive cells, goblet cells, regenerative cells and 
cells of the diffuse neuroendocrine system (DNES). The number of goblet cells increases from the cecum to the sigmoid colon, but the surface absorptive cells are the most numerous cell type. Rapid mitotic activity of the regenerative cells replaces the epithelial lining of the crypts and of the mucosal surface every 6 to 7 days. The mucosa is surrounded by a dense, irregular fibroelastic connective tissue layer, the submucosa. The muscularis externa consists of an outer longitudinal layer, which is not continuous along the surface but is gathered into three narrow ribbons of muscle fascicles, known as taeniae coli. The serosa envelops the muscularis externa and displays numerous fat-filled pouches, called appendices epiploicac. Histologically, the rectum resembles the colon, but the crypts of Lieberkühn are deeper and number fewer per unit area. ${ }^{91}$

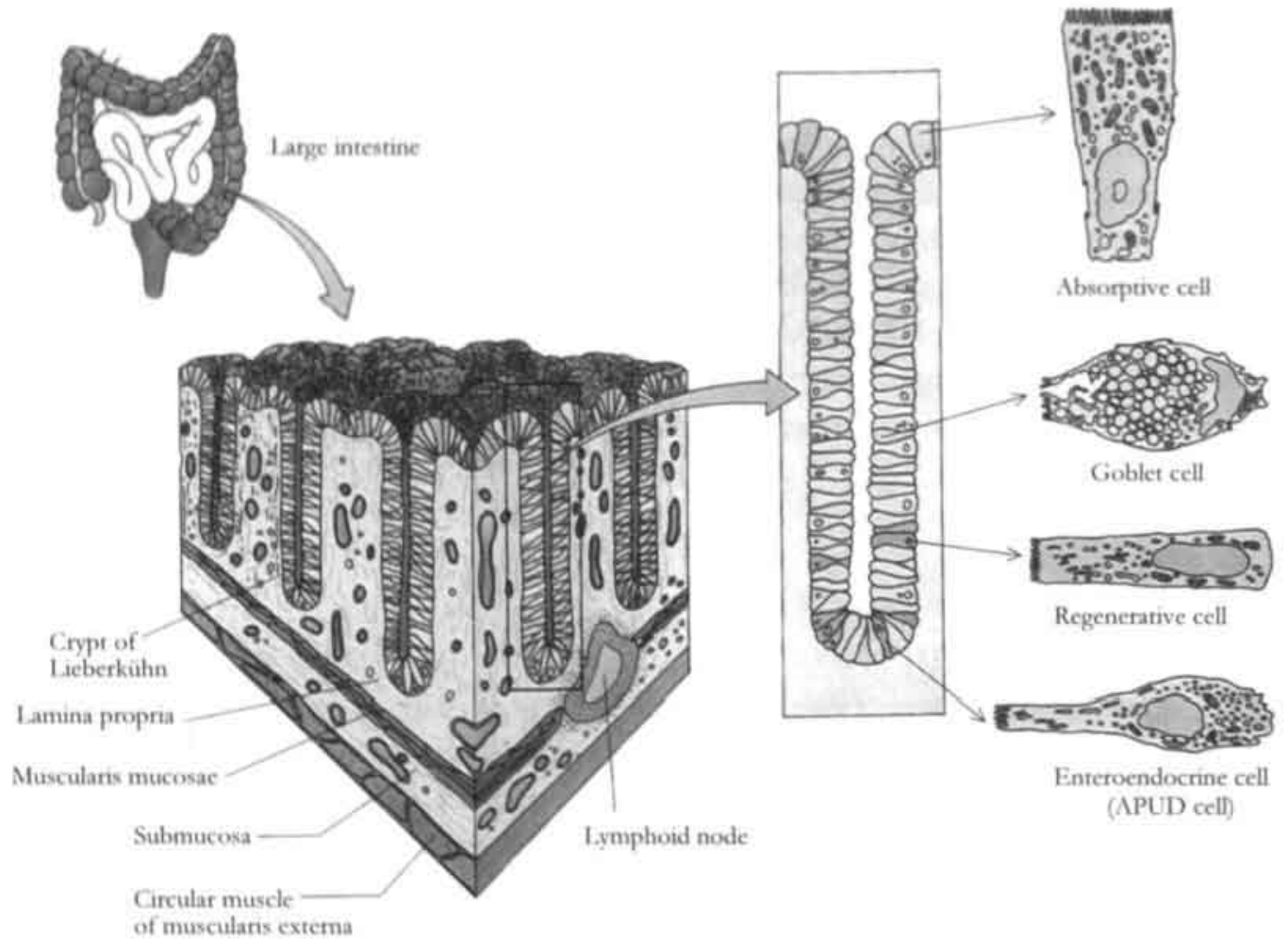

Figure 1. Schematic diagram of the colon, crypts of Lieberkuhn, and associated cells."

\subsubsection{Epidemiology and Etiology of Coloretal Cancer}

Worldwide, colorectal cancer (CRC) is the third most common incidence cancer and cause of death from cancer. Approximately 943,000 new cases were diagnosed and 491,000 people died of CRC in the year 2000. The peak incidence of the disease is in the seventh 
decade and it is fairly equally distributed berween men and women. Incidence rates vary around the world; the developed world (Europe, Japan, Australia, New Zealand and North America) accounts for almost 65 percent of the total global incidence. Central and South America, Asia and Africa are areas of low risk, however, the incidence is now increasing in these regions. ${ }^{8}$ Migrant and temporal trend studies show that rates in migrants from lowto high-risk countries tend to increase to the rates of the host countries within one or two generations, or sometimes even as early as within the migrating generation itself. This indicates that environmental factors play an important role in the etiology of CRC.92, 93

Increased CRC risk is associated with dietary (intake of excess fat, sugar and alcohol, low amount of vegetables and fiber) and lifestyle factors (low physical activity, high body mass, smoking), typical for Western societies. 8496 Based on epidemiological studies, Doll and Peto (1981) assessed that 90 percent of CRC mortality was attributed to dietary factors. ${ }^{97}$ This estimation was adjusted to 70 percent (range $50-80$ ) by Willet in $1995 .{ }^{98}$ CRC can largely be prevented by dietary changes. Especially diets containing considerable amounts of a variety of vegetables $(>400 \mathrm{~g} /$ day) may reduce the relative risk of CRC by 40 percent (range 30-50). ${ }^{99}$

Besides exogenous factors, CRC may be the result of hereditary factors or a combination of genetic and environmental factors. Patients with a familial risk - those who have two or more first- or second-degree relatives (or both) with CRC - make up approximately 20 percent of all patients with CRC, whereas about 5 percent of the total annual burden of CRC is caused by an autosomal dominant manner. This last category can be divided into two major forms of hereditary CRC, i.e., familial adenomatous polyposis (FAP) and hereditary nonpolyposis CRC (HNPCC). FAP (incidence $<0.5$ percent) is an autosomal dominant syndrome associated with mutations of the adenomatous polyposis coli (APC) gene, which results in the development of hundreds of polyps by the third decade of life. CRC inevitably occurs unless the colon is prophylactically removed. HNPCC is more common than FAP (incidence 4-6 percent) and is a much more heterogeneous syndrome with lifetime risk of 70-90 percent of developing CRC. It is caused by a germline mutation in any of the mismatch repair (MMR) genes hML.H1 (human mutL homolog 1), hMSH2, hMSH3 and hMSH6 (human mutS homolog 2, 3 and 6), hPMS1 or hPMS2 (human postmeiotic segregation 1 and 2). ${ }^{100-102}$ The experience derived from these two hereditary syndromes has been helpful in advancing our understanding of the molecular basis of CRC.

\subsubsection{Colorectal Cancer: Genetic Pathways involved in the Vogelstein Model}

For about a decade, the model proposed by Fearon and Vogelstein has been the paradigm of the genetic alterations involved in the development of CRC.103, ${ }^{104}$ In this model, CRC develops as the result of the progressive accumulation of genetic and epigenetic changes that lead to the transformation of normal colonic epithelium to colon adenocarcinoma, 
with the adenomatous polyp as an intermediate step in this sequence. The loss of genomic stability is an important step in this process by contributing to the occurrence of mutations.

Sporadic CRC arises mainly through two distinct pathways. In the first, chromosome instability, the initial inactivation of the APC tumor suppressor gene (chromosome $5 q$ ) is followed by the accumulation of alterations in additional oncogenes (K-RAS on chromosome 12p) and tumor suppressor genes (chromosome 18q: DCC, Deleted in Colorectal Cancer, chromosome 17p: TP53, p53 gene). The second, associated with microsatellite instability (MSI), occurs in $15-20 \%$ of sporadic CRC. Alterations have been found to cluster in genes encoding enzymes involved in the repair of DNA mismatches (in particular hML.H1 and hMSH2). These alterations provide a growth advantage and lead to clonal expansion of the distorted cells. ${ }^{103-107}$

Since the development of the Vogelstein model, a lot of information has become available on the function of the key genes in this model, as well as on their interactions. Additional genetic events have been identified and the specific molecular pathways which are perturbed by each of the mutations (Figure 2). Additionally, the importance of epigenetic mechanisms, like DNA methylation, that regulate gene expression is becoming increasingly clear. ${ }^{106-109}$ The genetic pathways, which are mostly investigated, are summarized below.

Wingless/Wnt signaling pathway: One of the central tumor-promoting effects of APC mutations is to lead to overactivation of the Wingless/Wnt signaling pathway, with the subsequent expression of genes that favor cell growth. APC mutations disrupt the association of APC with $\beta$-catenin, resulting in excessive amounts of $\beta$-catenin and overactivation of the Wnt signaling pathway. Consequently, genes that promote tumor formation are transcribed. Translocation of cytoplasmic $\beta$-catenin to the nucleus results in its interaction with other transcription factors like T-cell factor/lymphoid enhancing factor (TCF/Lef). TCF-4 is the predominant TCF family member expressed in colonic epithelium. Relevant targets up-regulated by TCF-4 identified to date include CYCLIN D1, C-MYC, MATRILYSIN, C-JUN, FRA-1, urokinase-type plasminogen activator receptor, and the peroxisome proliferator activator receptor delta (PPAR $)$ ). Other genes that are regulated by the Wnt signaling pathway and are upregulated in colon cancer include the WISP genes, WISP-1 and WISP-3. Consistent with the concept that overexpression of $\beta$ catenin is a central tumor-promoting effect of APC mutation, oncogenic mutations in the $\beta$-catenin gene (CTNNB1) have been observed in some CRC. ${ }^{106-109}$

K-RAS pathway: The K-RAS mutations appear to occur after APC mutations are formed and are associated with advanced adenomatous lesions. K-RAS pathways transduce signals from extracellular growth factors to regulate progression through the cell cycle and proliferation. K-RAS mutations are associated with upregulation of DNA methyltransferase, CYCLIN-D1, and GASTRIN. ${ }^{106-109}$ 
TGF- $\beta$ /SMAD pathway; Transforming growth factor beta (TGF- $\beta$ ) is a multifunctional cytokine that can induce growth inhibition, apoptosis, and differentiation in intestinal epithelial cells. The downstream transcriptional targets of the TGF- $\beta$ signaling pathways are the so-called SMAD proteins, which have been shown to regulate genes encoding plasminogen activator inhibitor 1 , the cyclin dependent kinase inhibitors p15, p21 and p27, cyclin D1, c-myc and TGF- $\beta$ itself. Mutational inactivation of SMAD2 and SMAD4 has been observed in a high percentage of pancreatic cancer and in 5-10\% of colon cancers. Next to DCC, these two tumor suppressor genes are the targets of $18 \mathrm{q}$ loss of heterozygosity (LOH). ${ }^{106-109}$

TP53 pathway: TP53 mutations appear to be late events in the colon adenomacarcinoma sequence that may mediate the transition from adenoma to carcinoma. It is expressed at very low levels in cells until it is activated via incompletely understood mechanisms by genotoxic stress. Its activation results in the transcription of genes that directly regulate cell cycle progression and apoptosis. These genes include $\mathrm{p}^{2} 1^{\mathrm{WAF}} / \mathrm{CIP}$, GADD45, MDM2, 14-3-3- $\sigma$, BAX, TSP1, KILLER/DR5, FAS/APO1, CYCLIN G, and others. Expression of many of these genes effectively halts DNA replication, induces DNA repair and inhibits angiogenesis, 106,107

DNA mismatch repair pathway: Somatic inactivation of the mismatch repair system additionally gives rise to approximately $15 \%$ of sporadic colon cancers. In either instance the resulting colon cancers display the phenotype of MSI. The predominant mechanism for inactivating MMR was found to be the epigenetic silencing of the hMLH1 promoter owing to aberrant promoter methylation. The most frequently targeted gene for mutation in this pathway is the TGF-b receptor type II tumor suppressor gene (TGFBR2). Other less frequently targeted genes include the IGF2 receptor, BAX, and CASPASE 5, genes encoding enzymes that regulate apoptosis; $\mathrm{E} 2 \mathrm{~F} 4$ and $\mathrm{TCF}-4$, transcription factors; MSH3 and MSH6, DNA mismatch repair genes; RIZ, the retinoblastoma proteininteracting zinc finger gene; and CDX2, an intestinal homeobox factor. Importantly, MSI and the subsequent target gene mutations appear to occur throughout the adenoma-tocarcinoma progression. ${ }^{100,}$, 105-107 


\section{COLORECTAL CANCER: GENETIC PATHWAYS INVOLVED IN THE VOGELSTEIN MODEL}

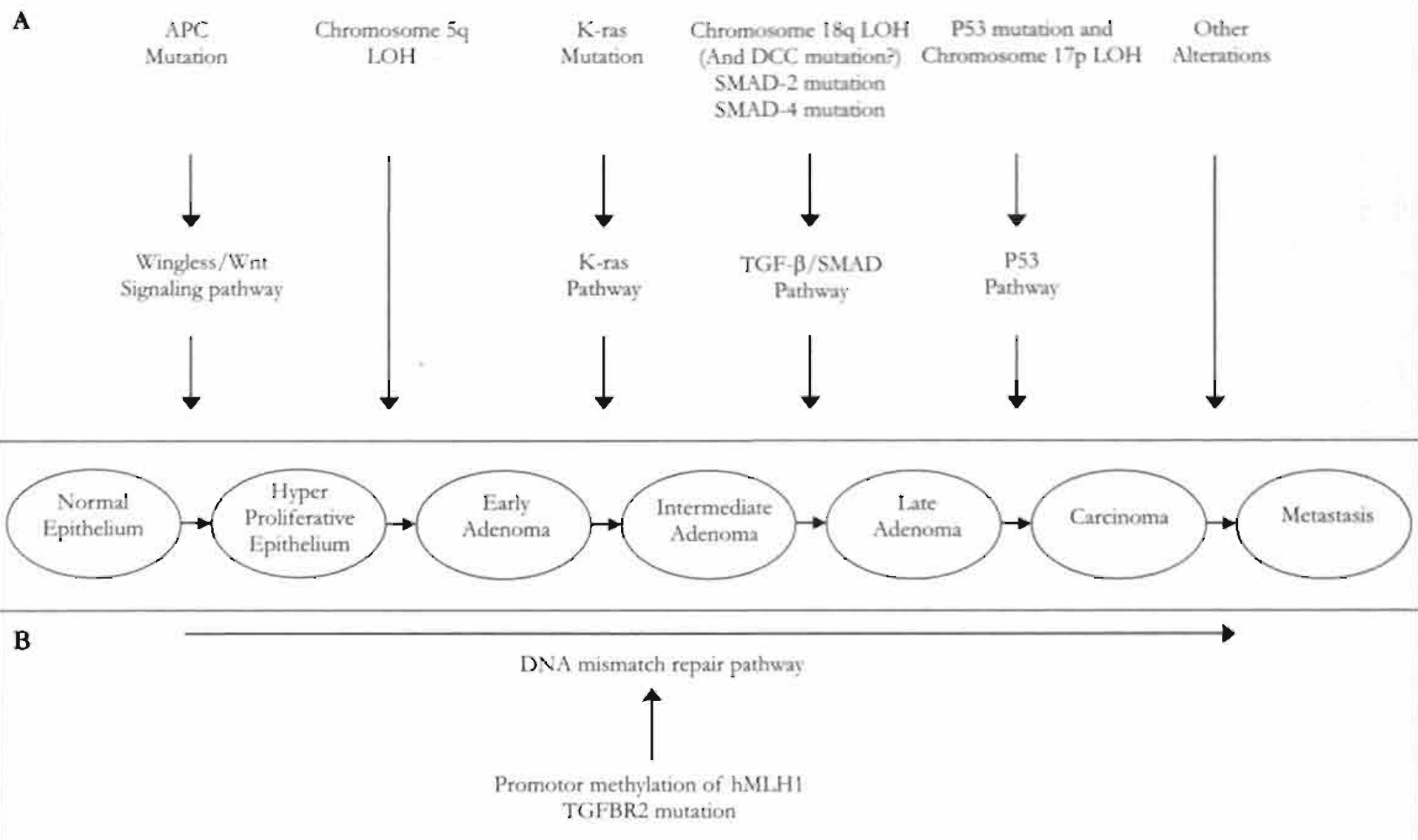

Figure 2. Overall scheme of key genetic eventx (genetic alterations and involved pathways) in colorectal tumotigenesta. The key genetic events can be grouped into two broad catagories. A) Eighty to eighty-five percent of tumors exhibit chromosomal instability. B) The remaining fifteen to twenty percent of tumors are characterized by mierosatellite instability. 


\subsubsection{Modulation of Colonetal Cancer (Risk) by Vegetables at the Genome Lavl}

Numerous studies have been conducted to get more insight into the mechanisms underlying prevention of CRC risk by vegetables or vegetable components. This paragraph will give an overview of a selection of in vitro-, animal-, and human studies in which the effect of vegetables or vegetable components on CRC risk is investigated, especially focusing on gene or protein expression changes; these are summarized in Table 4.

In vitro studies: Most of the in vitro studies involved in investigating the effect of vegetables on CRC risk use colon carcinoma cell lines as model system and one or more specific vegetable constituents as test compounds. Furthermore, they investigate the effect of these test compounds on cell proliferation, cell cycle arrest, apoptosis and the expression of genes or proteins involved in these processes. In these studies it was found that specific vegetable components such as flavonoids, indoles, isothiocyanates and $\beta$. carotene are able to induce cell cycle arrest and apoptosis and to reduce cell proliferation and that this coincides with specific modulation of genes and/or proteins, such as cyclins and caspases.68, 72, 75,76, 110

Animal studies: In animal studies, a limited number of studies has investigated the effect of vegetable or vegetable components on gene and/or protein expression changes in the colon. $28,31,39,111,112$ In these studies, modulation of (mostly biotransformation) genes and enzymes in the colon was observed. In most of the animal studies, the effect of vegetables or vegetable components on protein and gene expression is investigated in the liver $26,32,33,37,40,113$ and small intestine 26,113 or on other outcomes than protein and gene expression in the colon, such as development of intestinal neoplasia ${ }^{23,41,114,115}$ and aberrant crypt foci. ${ }^{24}$

Human studies: In only two human studies the effect is investigated of vegetables or vegetable components on modulation of the expression of genes or protein in the colon. ${ }^{111}, 116 \mathrm{Also}$, in other tissues such as lymphocytes 116,117 and urine ${ }^{118.120}$, the investigations are limited. More research has been done in the field of human dietary intervention trials in which the effect of nutritional interventions on the recurrence of precancerous polyps has been investigated. The results of these trials are, however, disappointing. 14, 121, 122 In most of the studies, no reduction of the number of adenomas was found after an intervention with vegetables or -components.

In conclusion, the number of in vitro, animal and human studies in which the effect of vegetables on gene expression and/or protein expression changes at target level is examined is limited. Most studies focus on surrogate tissues and only a few genes and/or proteins are investigated. Studies in which the effect of vegetables on the expression of multiple genes involved in various cancer preventive pathways in colon tissue are needed to elucidate the effects of vegetables. 
Table 4. Selection of in vitro, animal and human colon studies involved in modulation of gene or prorein expression by vegetables or vegetable cornponents.

\begin{tabular}{|c|c|c|c|c|}
\hline Model system & Vegetable or -component & Methods & Main results: & Ref. \\
\hline \multicolumn{5}{|l|}{ I) In vitro studies } \\
\hline \multirow[t]{2}{*}{ HT-29 cells } & Flavone $(150 \mu \mathrm{N})$ & $\begin{array}{l}\text { Cell proliferation-, cell } \\
\text { differentiation-, apoptosis } \\
\text { assays }\end{array}$ & $\begin{array}{l}\text { Reduced cell probiferation, induction of cell differentiation } \\
\text { and apoptosis }\end{array}$ & 73 \\
\hline & & RT-PCR analysis & $\begin{array}{l}\text { Inhibinion of } \mathrm{COX}-2, \mathrm{NF}-\mathrm{KB} \text {, cyclin } \mathrm{E} \text { and } \mathrm{B} \text {, anti-apoptotic } \\
\text { bch- } \mathrm{X}_{\mathrm{y}} \text {; Induction of } \mathrm{p} 21 \text {, pro-apoptotic bak; no change } \mathrm{p} 53\end{array}$ & \\
\hline \multirow[t]{2}{*}{ HT- 29 cells } & $\begin{array}{l}\text { Phenethyl isothiocyanate } \\
(5-50 \mu \mathrm{M})\end{array}$ & Apoptosis assays & $\begin{array}{l}\text { Induction of apoptosis; activation of caspase-3,-9; release of } \\
\text { cytochrome }\end{array}$ & no \\
\hline & . & Western blot analysis & $\begin{array}{l}\text { Inducrion of JNK, c-jun and } \mathrm{N} \text {-terminal kinase, extracellular } \\
\text { signal regulated protein kinase, } \mathrm{p} 38 \text { kinase }\end{array}$ & \\
\hline COLO 320 cells & $\beta$-carotene $(0-50 \mu \mathrm{M})$ & Cell growth inhibition & Inhibition of cell growth & 72 \\
\hline HSR cells & & Cell cycle analysis & Induction of cell cycle arrest & \\
\hline IS- 174 cells & & Apoptosis assay & Induction of apoptosis & \\
\hline HT-29 cells & & Immunhistochemistry & Inhibicion of cyclin $\mathrm{A}, \mathrm{Bcl}-2$ and bcl- $\mathrm{X}_{\mathrm{L}}$ no changes in $\mathrm{p} 21$ & \\
\hline WiDr cells: & & & and $\mathrm{p} 27$, and $\mathrm{Bax}$ & \\
\hline LS- 174 cells & Four different indoles. & Apoptosis assay & Induction of apoptosis & 76 \\
\hline \multirow[t]{4}{*}{ Caco -2 cells } & Three different isothiocyanates & Westem blot analysis & Induction of cytochrome P $4501 \mathrm{At}$ by the indoles & \\
\hline & (ICso conc.) & Northern blot analysis & Induction of AKRIC1, NQO1, and GCS by the & \\
\hline & & RT-PCR analysis & isorhiocyanates & \\
\hline & & Genotoxicity assay & Less DNA damage after pre-incubation & \\
\hline \multirow[t]{3}{*}{ HT- 29 cells } & Sulforaphane $(15 \mu \mathrm{M})$ & Apoptosis assay & Induction of apoptosis & $\Delta 8$ \\
\hline & & Cell cycle analysis & Induction of cell cycle arrest & \\
\hline & & Western blot analysis & $\begin{array}{l}\text { No change in p53, induction of pro-apoptotic bax, induction } \\
\text { of cyclin } A \text { and } B 1\end{array}$ & \\
\hline
\end{tabular}


Tuble 4. (Consinued) Sclection of in vitro, animal and human colon studies involved in modulavion of gene ar protein expression by vegetables or vegetable componenes.

\begin{tabular}{|c|c|c|c|c|}
\hline Model system & Vegetable or -component & Methods & Main results & Ref. \\
\hline \multicolumn{5}{|c|}{ II) Animal siudies } \\
\hline C57BI./6 mice & $\begin{array}{l}20 \% \text { soybean diet for } 14 \text { weeks; } \\
\text { or } 250 \mu \mathrm{g} \text { genistein orally (single } \\
\text { dose), sacrifice } 24 \mathrm{~h} \text { later }\end{array}$ & $\begin{array}{l}\text { Western blot analysis } \\
\text { RT-PCR analysis }\end{array}$ & $\begin{array}{l}\text { Induction of } \mathrm{CYP} 27 \mathrm{~B} 1 \text { and inhibition of } \mathrm{CYP} 24 \mathrm{mRNA} \text { and } \\
\text { protein expression after both the soybean diet and the } \\
\text { genistein gavage }\end{array}$ & 112 \\
\hline Wister rats & $\begin{array}{l}\text { Organosulfur compounds: diallyl } \\
\text { sulfide, diallyl disulfide, dipropyl } \\
\text { sulfide, or dipropyl disulfide } \\
(1 \mathrm{mmol} / \mathrm{kg} / \text { day) by gavage } \\
\text { (4days) }\end{array}$ & $\begin{array}{l}\text { Protein assays } \\
\text { Immunoblot assay }\end{array}$ & $\begin{array}{l}\text { Induction of UDP-glucuronyl transferase and microsomal } \\
\text { epoxide hydrolase by diallyl disulfide } \\
\text { Induction of quinone reductase and GST activiny by dially! } \\
\text { disulfide and diallyl sulfide. }\end{array}$ & $2 s$ \\
\hline $\begin{array}{l}\mathrm{C} 57 \mathrm{BL} / \mathrm{Gj} \\
\mathrm{Min} /+ \text { mice }\end{array}$ & $\begin{array}{l}\text { Diets for } 9 \text { weeks: } \\
\text { Caffeic acid phenethyl ester } \\
\text { (CAPE) }(0.03 \text { or } 0.15 \%) \text {; or } \\
\text { Curcumin }(0.1 \%) \text {; or Quercetin } \\
(2 \%) \text {; or Rutin }(2 \%)\end{array}$ & $\begin{array}{l}\text { Apoptosis analysis } \\
\text { Enterocyte proliferation. } \\
\text { assay } \\
\text { Immunhistochemistry }\end{array}$ & $\begin{array}{l}\text { In CAPE and curcumin fed mice } \\
\text { Decreased tumor formation } \\
\text { Increased enerocyte apoptosis } \\
\text { Proliferation of enterocyte back to normal levels } \\
\text { Inhibition of } \beta \text {-catenin protein expression in the enterocytes }\end{array}$ & "I \\
\hline Wister rats & $10 \%$ broccoli diet for 1 week & $\begin{array}{l}\text { Nylon blot analysis } \\
\text { Western blot analysis }\end{array}$ & $\begin{array}{l}\text { Induction of CYP1A1 mRNA and protein } \\
\text { Reduction of CYPIIB mRNA, induction of CYPIIB protein }\end{array}$ & 93 \\
\hline ICR(Ha) mice & $\begin{array}{l}\text { Lyophilized broccoli }(1 \mathrm{~g} / \mathrm{kg}) \\
\text { orally, xacrifice at } 1 \text { and } 2 \text { days } \\
\text { post-treatment }\end{array}$ & $\begin{array}{l}\text { Protein activity assay } \\
\text { Western blot analysis }\end{array}$ & $\begin{array}{l}\text { Induction of glutathione-S-transferase } \mu \text { and }-\pi \text { activity and } \\
\text { protein }\end{array}$ & 181 \\
\hline \multicolumn{5}{|c|}{ III) Human studies } \\
\hline $\begin{array}{l}\text { Human subjects } \\
\text { at increased risk }\end{array}$ & $\begin{array}{l}\text { Broccoli supplemented diet: } \\
\text { tablets }(6 \mathrm{~g} / \text { day }) \text { or Cruciferous } \\
\text { free diet for } 14 \text { days }\end{array}$ & Protein activity assay & No difference in GST- $\mu$ and - $\pi$ activity & in' \\
\hline $\begin{array}{l}\text { Healrhy human } \\
\text { subjects }\end{array}$ & $300 \mathrm{~g}$ Brussels sprours $/$ day & Westem blot analysis & Induction of GST $\mu$ and $-\pi$ levels in the rechum & 186 \\
\hline
\end{tabular}




\subsection{The Lungs}

\subsubsection{Histology of the Human Langs}

The lungs are part of the respiratory system and comprise the intrapulmonary bronchi (secondary and tertiary bronchi) primary bronchioles, terminal bronchioles, respiratory bronchioles and alveoli (Figure 3).

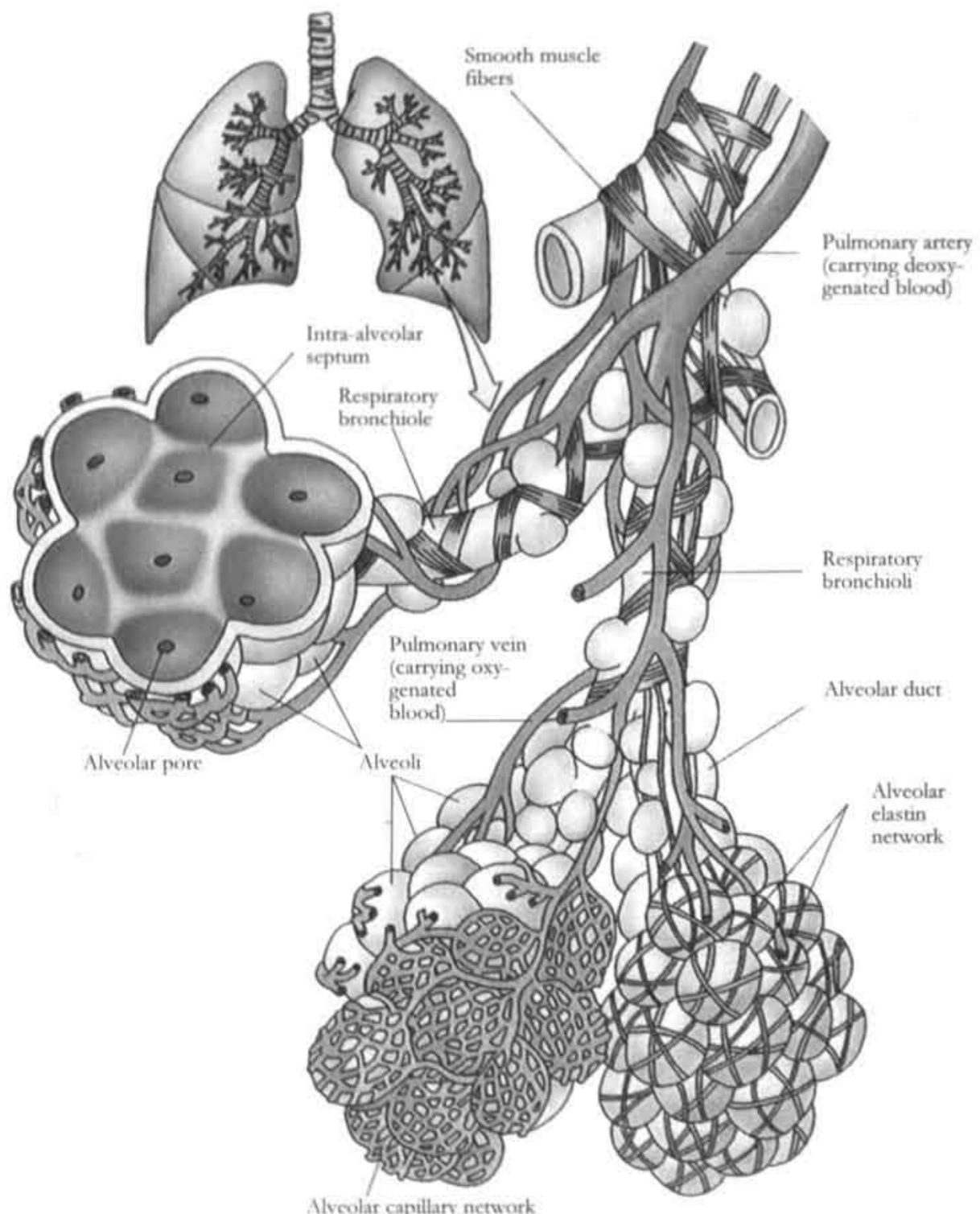

Figure 3. Schematic diagram of the respiratory system. 19 
The secondary bronchi lead to the lobes of the lung; the left lung has two lobes and thus has two secondary bronchi; the right lung has three lobes and thus has three secondary bronchi. As secondary bronchi enter the lobes of the lung, they subdivide into smaller branches, tertiary bronchi. Each tertiary bronchus splits and leads to a discrete section of the lung tissue known as bronchopulmonary segment. As they decrease in diameter, they eventually lead to bronchioles. ${ }^{123}$

The intrapulmonary bronchus consists of three layers: mucosa, submucosa, and adventitia. The mucosal lining is composed of the respiratory epithelium, the subepithelial connective tissue (lamina propria), and a bundle of elastic fibers separating the mucosa from the submucosa. The respiratory epithelium consists of six cell types: globet cells, ciliated columnar cells, basal cells, brush cells, serous cells, and DNES cells. Two distinct smooth muscle layers spiraling in opposite direction are located at the interface of the lamina propria and submucosa. The submucosa is composed of a dense irregular fibroelastic connective tissue housing numerous mucous and seromucous glands. The adventitia is also composed of fibroelastic connective tissue. The epithelial lining of bronchioles ranges from ciliated simple columnar with occasional globlet cells in larger bronchioles, to simple cuboidal with occasional Clara cells and no globlet cells in smaller bronchioles. The lamina propria of bronchioles has no glands, only smooth muscle cells which are surrounded by elastic fibers. Respiratory bronchioles are similar in structure to terminal bronchioles, but their wall is interrupted by the presence of alveoli, where gaseous exchange can occur. Each alveolus is a small outpouching of respiratory bronchioles, alveolar ducts, and alveolar sacs. Walls of alveoli are composed of squamous epithelium consisting of two types of cells: type-I pneumocytes and type-II pneumocytes. ${ }^{123}$

\subsubsection{Epidemiology and Etiology of L sung Cancer}

Lung cancer is the most common malignant disease worldwide, and is the major cause of death from cancer. It was a rare disease until the beginning of the $20^{\text {th }}$ century. Since then, the occurrence of lung cancer has increased rapidly and it now accounts for approximately 902,000 new cases each year among men and 337,000 among women. An estimated $1,100,000$ people died of lung cancer in the year 2000.8. In both men and women, the incidence is low before age 40, and increases up to at least 70 . The difference in incidence between men and women correlates with the difference in overall pattern of smoking tobacco, which is the predominant risk factor. However, for the same lifetime exposure to tobacco, risks are almost similar in men and women. In men, more than 80 percent of lung cancer cases are caused by smoking; in women the attributable risk is less: about 70 percent in Northern Europe, 45 percent worldwide. 8.124 The geographical and temporal patterns of lung cancer incidence are overwhelmingly determined by the use of tobacco. The highest incidence rates are recorded in North America, Europe, Japan, Australia, New Zealand and China and lowest in South America, Southern Asia and Africa. ${ }^{125}$ 
Next to smoking, the other causes of lung cancer are known: environmental tobacco smoke (passive smoking); occupational exposures to asbestos, arsenic, chloromethyl ethers, chromium-VI, and nickel; residential and occupational exposures to radon; and exposures to carcinogenic air pollutants. ${ }^{124-126}$ Furthermore, risk factors include dietary factors 124,127 and genetic factors. ${ }^{128-130}$

In 1981, Doll and Peto assessed that 20 percent of lung cancer mortality was attributed to dietary factors. ${ }^{97}$ Willet (1995) came to the same conclusion after revising the epidemiological studies and taking into account the studies published since. ${ }^{98}$ Lung cancer can be prevented by dietary changes. Diets high in vegetables and fruits convincingly decrease risk; diets high in selenium, vitamin $\mathrm{C}$ and $\mathrm{E}$ possibly and diets high in carotenoids probably decrease risk; whereas diets high in total fat and alcohol possibly increase risk ${ }^{124,127}$ (Table 1). Especially diets containing considerable amounts of a variety of vegetables $\left(>400 \mathrm{~g} /\right.$ day) may reduce the relative risk of lung cancer by 45 percent. ${ }^{99}$

Evidence from studies of familial aggregation of lung cancer suggests that genetic factors are involved in human lung tumor development. A positive familial history of lung cancer has been identified as a risk factor. ${ }^{131}$ More specifically, segregation analysis of lung cancer pro-band families indicates that a Mendelian codominant inheritance of a rare major autosomal gene is involved. ${ }^{132}$ In addition to the gene at this locus, several other genetic factors have been linked to lung cancer susceptibility. Genetic variants or polymorphisms have been identified that are hypothesized to affect lung cancer risk. In particular, genetic differences in the genes (CYP2D6, CYP1A1 and GSTM1) responsible for metabolism of tobacco carcinogens have been implicated in susceptibility of lung cancer. ${ }^{133,134}$ Finally, lung cancer also appears at an increased rate in several genetic syndromes including the $\mathrm{Li}$ Fraumeni syndrome, hereditary retinoblastoma, familial breast cancer, and the Bloom syndrome, ${ }^{129}$

\subsubsection{Lung Cancer: Genetic Altenations and Imvolved Patbways}

During the past two decades, considerable efforts have been made to the identification of the genes that drive lung cancer development. Histologically apparent lung cancer is due to the sequential accumulation of specific genetic and morphologic changes to the normal epithelial cells of the lung. The major histological types of lung cancer are 1) non-small cell lung cancers (NSCLC), which represent about 80 percent of lung cancers and are divided into squamous cell carcinoma, adenocarcinoma, large cell carcinoma and mixed types, and 2) small cell lung cancers (SCLC), which represent about 20 percent of lung cancers. Some of the genetic alterations are specific to one of the two major lung cancer types and there are those that are common in both. ${ }^{128,135-137}$ Until now, no single or combination of genetic events has been identified in 100 percent of lung cancer tissues.

Table 5 gives an overview of the main genetic alterations in lung cancer and the relative abundance in NSCLC and SCLC, respectively. ${ }^{128}$ Particularly, these comprise 
mutations or deletions of tumor suppressor genes (resulting in inactivation) and protooncogenes (resulting in activation), and epigenetic inactivation of these genes via DNA methylation (hypermethylation of gene promoter regions). The role of these genes and their involved pathways are shortly described.

Changes in several tumor suppressor genes are related to the development of lung cancer, including the APC and TP53 (See paragraph 2.2 .3 for involved genetic pathways). Distict from CRC, $A P C$ is not mutated in lung cancer, but it is inactivated through hypermethylation of the promoter region. ${ }^{128,136,137}$

The p16-cyclin D1-cyclin-dependent kinase 4 (CDK4)-retinoblastoma pathway is disturbed in both SCLC and NSCLC. This pathway is eentral to controlling the $\mathrm{G} 1$ to $\mathrm{S}$ phase transition of the cell cycle. One of the four gene products that comprise this pathway is mutated or functionally altered in many human cancers; the two tumor suppressor gene products affected in lung cancer are p16 in NSCLC and RB in SCLC. Cyclin D1 overexpression can coexist with these changes, RB binds to the E2F transcription factor and therefore E2F cannot activate genes needed to initiate the S phase. Moreover, this complex also represses the transcription of other target genes. Phosphorylation of RB by cyclin D1/CDK4-6 releases E2F, which initiates the S phase. This pathway can be turned on by mutations inactivating RB, inactivating p16, overexpression of cyclin D1, or overexpression of CDK4. Other tumor suppressor genes affected in lung cancer are, for example, the fragile histidine triad gene (FHIT), RASSF1A, and $\mathrm{CDH} 13$. These genes undergo promoter hypermethylation, causing loss of expression. Other tumor suppressor genes present on chromosome 3p are FUSI, SEMA3B, 101F6, and NPRL.2 which are affected by LOH. ${ }^{128,134-137}$

Next to changes in tumor suppressor genes, proto-oncogenes are affected in such a way that cell growth is stimulated. Furthermore, autocrine and paracrine growth factor stimulatory loops exist in lung cancer. Several, but not all components of these stimulatory pathways are proto-oncogene products. An important positive signaling pathway is gastrinreleasing peptide (GRP) and its receptor family. This growth stimulatory loop has a role in lung development and repair, but it also becomes 'reactivated' in lung cancer, particularly SCLC. Another important autocrine loop is the stem cell factor/tyrosine kinase receptoroncogene KIT (SCF/KIT), also active in SCLC. An autocrine loop specific for NSCLC is the hypocyte growth factor/metastasis proto-oncogene (HGF/MET). The HGF stimulates mitogenesis or motogenesis of human bronchial epithelial, alveolar type II. Furthermore, NSCLC demonstrate abnormalities of the neuregulin receptors, ERBB1 and ERBB2, which are part of a family of transmembreane receptor tyrosine kinases. On ligand binding, ERBB receptors homo- or heterodimerize, thereby inducing intrinsic kinase activities that initiate intracellular signal transduction cascades, which regulates epithelial proliferation and differentiation. Overexpression of ERBB correlates with increased tumorigenicity of bronchial epithelial cells and metastatic potential of NSCLC. 128,136 
K-RAS is the most frequently activated RAS gene in lung cancer, resulting in inappropriate signaling for continued cell division (See involved pathway paragraph $2.2 .3) .128,130,136,137$ RAS signaling ultimately activates nuclear proto-oncogenes such as MYC, which, on heterodimerization, transcriptionally activates downstream genes that dive cells to grow. Activation occurs by gene amplification or by transcriptional dysregulation, both leading to protein overexpression. ${ }^{128,} 130,136,137$

Other changes relevant in lung carcinogenesis are increased BCL-2 expression, telomerase activity, microsatellite instability and promoter hypermethylation. The product of the breakdown cluster lymphoma (BCL.2) anti-apoptotic proto-oncogene is the key player in apoptosis, highly expressed in SCLC, making it less sensitive for apoptosis. ${ }^{12 \mathrm{k}, 136}$

Table 5. Major genetic alterations in lung cancer. ${ }^{\text {t2s }}$

\begin{tabular}{|c|c|c|}
\hline & $\operatorname{SCLC}(\%)$ & NSCLC (\%) \\
\hline \multicolumn{3}{|l|}{ Tumor suppressor genes } \\
\hline \multicolumn{3}{|l|}{ P53 abnormalities } \\
\hline Mutation with $17 \mathrm{p} 131 \mathrm{OH}$ & $75-100$ & -50 \\
\hline Abnormal p 53 expression & $40-70$ & $40-60$ \\
\hline \multicolumn{3}{|l|}{ P16-cyclin DI.CDK4-RB pathway lesions } \\
\hline $\begin{array}{l}\text { P16 mutation or DNA methylation with } \\
9 \text { p21 LOH }\end{array}$ & $<0.1$ & $10-40$ \\
\hline Absent p16 expression & $0-10$ & $30-70$ \\
\hline Absent RB expression with $13 \mathrm{q} 14 \mathrm{LOH}$ & $\sim 90$ & $15-30$ \\
\hline APC $(5 q 211 . O H)$ DNA methylation & 26 & 46 \\
\hline Chromosome 3p LOH (several sites) & 100 & 90 \\
\hline RAR-B 3p24 (DNA methylation) & 76 & 40 \\
\hline RASSF1A 3p21.3 (DNA methylation) & 90 & $30-40$ \\
\hline FHIT 3p14.2 (DNA methylation and deletion) & 64 & $\sim 50$ \\
\hline CDH13 (DNA methylation) & 20 & 45 \\
\hline \multicolumn{3}{|l|}{ Proto-oncogenes and growth stimulation } \\
\hline \multirow[t]{2}{*}{ Putative autocrine loops } & GRP/GRP receptor & $\mathrm{HGF} / \mathrm{MET}$ \\
\hline & $\mathrm{SCF} / \mathrm{KIT}$ & NDF/ERBB \\
\hline RAS mutation & $<1$ & $15-20$ \\
\hline $\mathrm{MYC}$ amplification & $15 \cdot 30$ & $5-10$ \\
\hline \multicolumn{3}{|l|}{ Other changes } \\
\hline $\mathrm{BCl} 2$ expression & $75-95$ & $10-35$ \\
\hline Telomerase activity & -100 & $80-85$ \\
\hline Mficrosatellite instability & $\sim 35$ & -22 \\
\hline Promoter hypermethylation & marker development & marker development \\
\hline
\end{tabular}

Abbreviations: LOH: loss of heterozygosary; CDK4: cyclin-dependent kinase 4; RB: retinoblastoma; APC: adenomatous polyposis coli; RAR-B: retinoic acid receptor B; RASSF1A: tumor suppressor RASSF1 isoform A; FHIT: fragile histidine triad; CDH13; cadherin 13 (H-cadherin); GRP: gastrin releasing receptor; SCF: stem cell factor, KIT: tyrosine kinase receptoroncogene KIT, HGF: hepatocyte growth factor; MET: metastasis oncogene; NDF: new differentianon factor; FRBB: ERBB1: epidemal growth factor teceptor, ERBB2: Her2/neu. 


\subsubsection{Modulation of Lung Caneer (Risk) by V egetables at the Genomo Level}

Although the evidence from epidemiological studies is fairly convincing that vegetables and/or vegetable components decrease the risk of lung cancer, a small amount of experimental studies have been conducted in lung tissues or lung cells exposed to vegetables or vegetable components. This paragraph will give an overview of a selection of in ritro-, animal-, and human studies in which the effect of vegetables or vegetable components on lung cancer risk is investigated. Studies focusing on gene or protein expression changes are of particular interest; these are summarized in Table 6.

In vitro studies: A few studies have examined the effect of vegetable components on gene and/or protein expression in lung cells ${ }^{138,139}$, thereby solely focusing on effects on cell cycle regulation. Also, other indicatives of lung cancer risk have not been examined in large amounts in in vitr lung systems.

Animal studies: In animals, a limited number of studies has investigated the effect of vegetable or vegetable components on gene and/or protein expression changes in the lung. $28,140,141$ However, in these studies, modulation of (mostly biotransformation) genes and enzymes in the lung was detected. As already stated in paragraph 2.2 .4 , in animals, the effect of vegetables or vegetable components on protein and gene expression is investigated in the liver ${ }^{26,32,33,37,40.113}$ and small intestine ${ }^{26,}, 113$ or on other outcomes than protein and gene expression in the colon, such as development of lung tumors..$^{142-144}$

Human studies: According to literature review, no human studies have investigated the effect of vegetables or vegetable components on modulation of the expression of genes or protein in the lung (medline, accessed July $3^{\text {rd }}$ 2004). As explained in paragraph 2.2.4, the investigations in other tissues such as lymphocytes 116,117 and urine ${ }^{118-120}$, are limited. More research has been done in the field of human dietary intervention trials in which the effect of nutritional interventions on the occurrence of lung cancer has been investigated. The results of these trials are, as in colon cancer prevention trials, however, unacceptable. $126,127.145-147$

In summary, the number of in vitro and animal studies in which the effect of vegetables on gene expression and/or protein expression changes at target level is examined is limited. No human studies have been reported in which the effect of vegetables or vegetable components on intermediate biomarkers for lung cancer is investigated. In addition, results of human intervention trials measuring inhibiting effects of vegetables on lung cancer occurrence, are disappointing. Again, more research is desired in which the effect of vegetables on the expression of multiple genes involved in various cancer preventive pathways is investigated, to give more insights into the genetic mechanisms underlying lung cancer prevention. 
Table 6. Selection of in vitro, animal and human lung studies involved in modulation of gene or procein expression by vegetables or vegetable components.

\begin{tabular}{|c|c|c|c|c|}
\hline Model system & Vegetable or -component & Methods & Main results & Ref. \\
\hline \multicolumn{5}{|l|}{ I) $I n$ vitro studies } \\
\hline $\begin{array}{l}\mathrm{C} 10, \mathrm{E} 9 \text {, and } 82 \text {. } \\
132 \text { murine lung } \\
\text { epithelial cells } \\
\mathrm{C} 3 \mathrm{H} 10 \mathrm{~T} 1 / 2 \\
\text { murine fibroblasts }\end{array}$ & $\begin{array}{l}\text { B-carotene }(1-10 \mu \mathrm{M}, 1-5 \text { days } \\
\text { treatment) }\end{array}$ & $\begin{array}{l}\text { Growth inhibition assays } \\
\text { Gap junctional intercellular } \\
\text { communication (GJIC) assay } \\
\text { Protein assay }\end{array}$ & $\begin{array}{l}\text { No effect on growth, GIIC and connexin } 43 \text { protein } \\
\text { expression in C10, E9 and } 82-132 \text { cells } \\
\text { Growith reduction, enhancement of GIIC and connexin } 43 \\
\text { protein expression in C3H10T1/2 munine fibroblasts }\end{array}$ & $w$ \\
\hline $\begin{array}{l}\text { A } 549 \text { hurnan lung } \\
\text { adenocarcinoma } \\
\text { cells }\end{array}$ & $\begin{array}{l}\text { Flavone }(30,60 \text { and } 100 \mu \mathrm{M} \text {, } \\
\text { for } 24,48 \text { or } 72 \mathrm{~h})\end{array}$ & $\begin{array}{l}\text { Cell growth assay } \\
\text { Cell cycle analysis } \\
\text { Western blot analysis } \\
\text { Northern blot analysis }\end{array}$ & $\begin{array}{l}\text { Inhibinon of cell growth } \\
\text { Cell cycle arrest } \\
\text { Induction of } \mathrm{p} 21 \mathrm{mRNA} \text { and protein } \\
\text { Inhibition of phosphorylation of Retinoblastoma protein }\end{array}$ & 198 \\
\hline \multicolumn{5}{|l|}{ II) Animal studies } \\
\hline Wister rats & $\begin{array}{l}\text { Organosulfor compounds: } \\
\text { diallyl sulfide, diallyl disulfide, } \\
\text { dipropyl sulfide, or dipropyl } \\
\text { disulfide ( } 1 \mathrm{mmol} / \mathrm{kg} \text { ) by } \\
\text { gavage daily ( } 4 \text { days) }\end{array}$ & $\begin{array}{l}\text { Protein assays } \\
\text { Imumunoblot assay }\end{array}$ & $\begin{array}{l}\text { Induction of UDP-glucuronyl transferase, microsomal } \\
\text { epoxide hydrolase, GST activity and quinane reductase by } \\
\text { dallyl disulfide }\end{array}$ & 3 \\
\hline Sprague-Dawly rats & $\begin{array}{l}\text { Glucoraphanin ( } 120 \text { or } 240 \\
\mathrm{mg} / \mathrm{kg} \text { ) by gavage in a single } \\
\text { dose or daily during } 4 \text { days }\end{array}$ & $\begin{array}{l}\text { Protein assays } \\
\text { ESR technique }\end{array}$ & $\begin{array}{l}\text { Induction of UDP-glucuronylmansferase and GST } \\
\text { Induction of CYP enzymes } \\
\text { Induction of reactive oxygen species }\end{array}$ & 100 \\
\hline $\mathrm{A} / \mathrm{J}$ mice & $\begin{array}{l}\text { Non-oxidative vitamin E (100 } \\
\mathrm{mg} / \mathrm{kg}) \text { single dose }\end{array}$ & $\begin{array}{l}\text { Cell proliferation assay } \\
\text { Northern blot analysis } \\
\text { Protein assay }\end{array}$ & $\begin{array}{l}\text { Reduced cell proliferation } \\
\text { Inhibition of ornithine decarboxylase activity and } \\
\text { reduction of mRNA }\end{array}$ & 141 \\
\hline
\end{tabular}


It is clear from paragraph 2.2 and 2.3 that in colorectal as well as in lung cancer development a lot of gene modulations are involved. However, the involved genetic pathways by which vegetables exert their beneficial effect, are grossly unknown. Therefore, more research is needed, in which the effects of vegetables on multiple genes representing different genetic pathways, are investigated. This can be achieved by using microarray technology, which enables rapid examination of the expression of hundreds to thousands of genes in a single experiment. This technology, together with its implication in nutritional research, is explained in paragraph 3.

\section{DNA Microarray Technology in Food and Nutrition Sciences}

\subsection{Gene Expression Profiling}

The profile of genes expressed by different cells determines their phenotype, and thus provides insights into the molecular basis for health and disease. Changes in the multi-gene pattern of expression can provide clues about regulatory mechanisms and broader cellular functions and biochemical pathways. Various methods are available for detecting and quantitating gene expression levels, including northern blots ${ }^{148}$, differential display ${ }^{149}$, reverse transcription polymerase chain reaction (RT-PCR) ${ }^{150,} 151$ and serial analysis of gene expression (SAGE). ${ }^{152}$ The advent of the DNA microarray technology as the newest technology on gene expression measurements has enabled rapid and simultaneous comparison of mRNA levels for multiple genes in a single experiment. The use of DNA microarrays to study gene expression profiles of biological samples began in $1995 .{ }^{153}$ Since that time, the technology required for making and using arrays, research fields applying the technology and statistical tools for data analyses have been extensively reviewed. ${ }^{154-161}$

\section{$3.2 \quad$ Nutrigenomics}

The DNA microarray technique has been increasingly utilized in various fields of life sciences. In nutrition research, microarrays have already been used to study retardation of aging by caloric restriction ${ }^{162}$, effects of high-fat diet ${ }^{163}$, effects of isoflavones ${ }^{164}$, and obesity and diabetes. ${ }^{165}$. This new research area is termed nutritional genomics or nutrigenomics. The fundamental concepts of the field are that the progression from a healthy phenotype to a chronic disease phenotype must occur by changes in gene expression or by differences in activities of proteins and enzymes, and that dietary chemicals directly or indirectly regulate the expression of genomic information. ${ }^{160}$ Applied 
wisely, it will promote an increased understanding of how nutrition influences metabolic pathways and homeostaticcontrol, how this regulation is disturbed in the early phase of a diet-related disease and to what extent individual sensitizing genotypes contribute to such diseases. The ultimate goal of nutrigenomics is to allow effective dietary-intervention strategies to recover normal homeostasis and to prevent diet-related diseases. ${ }^{167}$

\subsection{Proteomics}

A complementary technology to DNA microarrays for monitoring gene expression is provided by proteomics, a term generally used to encapsulate the quantitation of all proteins, the determination of their post-translational modifications and how these are dependent on cell state and environmental influences. Proteins are frequently functional molecules and could therefore provide a better view to understanding gene function and reflecting the post-transcriptional regulation level. Some messengers are transcribed but not translated, and the number of mRNA copies does not necessarily reflect the number of functional protein molecules. ${ }^{168}$ However, for most genes, changes in mRNA abundance are related to changes in protein abundance. ${ }^{169,170}$ Furthermore, protein-based approaches are generally more difficult, less sensitive and have a lower throughput than RNA-based ones. Many methods have been developed for monitoring protein levels either directly or indirectly. Currently, protein screening and detection is being performed by twodimensional polyacrylamide gel electrophoresis (2-DE) coupled with annotation of the detected proteins by mass spectroscopy, usually matrix-assisted laser desorption ionization mass spectroscopy (MALDI-MS). Automated methods have been devised for running several gels in parallel but the major drawback to this method is the time needed for annotating the proteins by mass spectroscopy and comparing sequences back to expressed sequence tag (EST) databases. To solve this problem, newer methods have been developed using protein microarrays, although these are relatively expensive and the number of proteins present on these arrays is still limited. ${ }^{169}$ The importance of the protein-based methods is that they measure the final expression product rather than an intermediate. In addition, some of them enable the detection of post-translational protein modifications and protein complexes, and in some cases, yield information about protein localization, none of which are obtained directly by measurements of mRNA. ${ }^{171}$

\subsection{Microarray Platforms}

The basic concept behind all DNA microarrays is the precise positioning of DNA fragments (probes) at high density on a solid support so that they can act as molecular 
detectors. In practice, DNA microarrays vary according to the solid support used (such as glass or filters), the surface modifications with various substrates, the type of DNA fragments on the array (such as CDNA, short ( -25 base) or long ( $50-80$ base) oligonucleotides or genomic fragments), whether the gene fragments are presynthesized and deposited or synthesized in situ, and the machinery used to place the fragments on the array (such as ink-jet printing, spotting, mask or micromirror-based in situ synthesis). Currently, combinations of these variables are used to generate different types of DNA microarrays: filter arrays, spotted glass slide arrays, and in situ synthesized oligonucleotide arrays. ${ }^{172.174} \mathrm{~A}$ key consideration is whether to adopt a do-it-yourself approach and spot arrays in the laboratory or to purchase arrays from a commercial supplier.

\subsection{Microarrays used in the presented Studies of this Thesis}

For the human study (Chapter 2), gene expression analysis was carried out using the commercially available PHASE-1 Microarray Human-600 (PHASE-1 Molecular Toxicology, USA), which contain 597 sequence verified human genes, representing a number of biologically relevant and control genes. The genes were selected based on relevance for responses of cells to xenobiotic compounds and knowledge of the involved processes. The presented pathways relate to apoptosis, cell cycle, cell proliferation, DNA damage and repair, inflammation, metabolism, oxidative stress, peroxisome proliferators, transport, cell environment, etc. These pathways and involved genes play an important role in cancer development. Target genes are single stranded, approximately 500 nucleotides in length and spotted in quadruplicate on glass microscope slides (Table 7).

For the mouse studies (Chapter 3-6), a mouse cDNA microarray was constructed based on the genes present on the PHASE-1 Microarray Human-600.

\section{Table 7. Gene Categories present on the PHASE-1 Human 600 microarray.}

\begin{tabular}{|ll|}
\hline Gene Categories & Types of Genes in Category \\
\hline Apoptosis & Caspases, BAK, Bax, Fas, Cyclins, TNFs \\
Cell Cycle & Cyclins, DNA Binding Protein, Waf 1 \\
Cell proliferation & Kinases, Transcription Factors, Growth Factors and Receptors, Connexins \\
DNA Damage/ Repair & DNA Repair Genes, ERCC's, GADDs, Helicases, Topoisomerases \\
Inflammation & Serum Amyloids, Interleukins, Adhesion Molecules, Chemokines \\
Metabolism & P450s, Glucuronidation and Glutathione Enzymes, Methyltransferases \\
Oxidative stress & $\mathrm{O}_{2}$ Response Genes, Superoxide Dimutase, Redox Enzymes \\
Peroxisome Proliferators & Peroxisomal Enzymes \\
Transport & Multi-drug Resistance Proteins, Organic Anion and Cation Transporters \\
Cell-environment & Connexins, Integrins, Selectins, Cadherins \\
\hline
\end{tabular}




\section{$4 \quad$ Aim and Outline of the Thesis}

It is clear from both epidemiological and experimental studies, that regular consumption of vegetables decreases the risk of cancers at many target sites. Convincing evidence exists for the gastrointestinal tract (oral cavity, pharynx, esophagus, stomach, colon and rectum) and respiratory tract (lung). The epidemiological evidence, however, has recently weakened and there are many gaps in our knowledge. Numerous potentially anticarcinogenic agents are present in vegetables, which have various potential mechanisms of action. In many of these postulated mechanisms, modulation of gene expression plays a role. However, this has only been investigated for a limited number of genes and mostly applied to pseudotarget cells like lymphocytes instead of the ultimate target organ. The molecular targets at the genome level are mostly unknown. Technologies are being developed that allow the simultaneous determination of the expression of many thousands of genes at the mRNA (transcriptomics) and protein (proteomics) levels. These tools make it possible to assess the effect of a specific diet on the expression of multiple genes and proteins simultaneously. It can help to identify and characterize the basic molecular pathways of gene regulation by vegetables. The hypothesis investigated in the present thesis is that an important contribution of the anticarcinogenic effects of vegetables in the colorectum and lung is through modulating the expression of genes involved in biological and genetic pathways that are relevant for carcinogenesis. Therefore, the aim is to provide more insight into the molecular mechanisms by which vegetables exert their anticarcinogenic effects, by focusing on the effects at the genome level in the relevant target cells.

Therefore, the research topics of the present thesis are:

To identify genes and involved pathways which are modulated by vegetables in vivo in colorectal mucosa from healthy female volunteers and sporadic adenoma patients; and to study if sporadic adenoma patients, who are at higher risk for developing colorectal cancer, respond differently compared to healthy controls; To investigate the dose-dependent effect of a mixture of vegetables on gene expression in the colon mucosa of C57BL6 female mice, to mimic the human dietary intervention study in which two different doses of a vegetable mixture were used;

To investigate the dose-dependent effect of vegetables on protein expression in the colon mucosa of C57BL6 female mice;

To study the contribution of four different vegetables on gene expression in colon mucosa of C57BL6 female mice; 
To investigate the systemic effect of different doses of a vegetable mixture and the role of the four specific vegetables in this mixture on gene expression in lung tissue of C57BL.6 female mice.

The outline of the thesis is as follows:

Chapter 2: Adenoma patients are at higher risk for developing colorectal cancer compared to healthy subjects. This chapter describes the results of a human dietary intervention study in which the effect of a decreased or increased intake of a mixture of vegetables (cauliflower, carrots, peas and onions) during two weeks on gene expression was investigated in biopsies from normal colorectal mucosa of female healthy volunteers and sporadic adenoma patients by applying cDNA microarray technology. Similarities and differences are described.

Chapter 3: The C57BL6 mouse model is frequently used to study the effect of diet and/or gene expression. To investigate whether the effect of vegetables on gene expression changes in the colon is dose dependent, C57BL.6 female mice were fed one of four different diets containing different doses of a vegetable mixture consisting of the same combination and type of vegetables used in the human dietary intervention study.

Chapter 4: A proteomics approach was followed to analyze the proteome changes induced in the colon mucosa of the female C57BL6 mice the vegetable mix study described in Chapter 3.

Chapter 5: Individual vegetables can have a particular effect on gene expression. Therefore, the role of the four specific vegetables used in the mix study described in Chapter 4 on gene expression in the colon is investigated in female C57BL6 mice.

Chapter 6: From epidemiological evidence it is known that lung cancer can be protected by vegetables. The effects of vegetables on gene expression in this organ are studied in the same female C57BL6 mice described in Chapters 3 and 5. The dose dependent effect of the mixture and the contribution of the specific vegetables are discussed.

Chapter 7: The main findings of the studies described in this thesis are discussed and summarized. 


\section{References}

1. Steinmetz KA, Potter JD. Vegetables, fruit, and cancer prevention: a review. J Am Diet Assoc 1996;96:1027-39.

2. Fenwick GR, Heaney RK, Mullin WJ. Glucosinolates and their breakdown products in food and food plants. Crit Rev Food Sci Nutr 1983;18:123-201.

3. Steinmetz KA, Potter JD. Vegetables, fruit, and cancer. I. Epidemiology. Cancer Causes Control 1991:2:325.57.

4. Food, Nutrition and the Prevention of Cancer: a Global Perspective. Chapter 6.3: Vegetables and Fruits, World Cancer Research Fund \& American Institute for Cancer Research. Washington: BANTA Book group, 1997:436-446.

5. Bennion M. Chapter 14: Vegetables, In: The science of food. New York: John Wiley \& Sons, 1980:217-244.

6. Stocks P. Kam MN. A co-operative study of the habits, home life, dietary and family histories of 450 cancer patients and of an equal number of control patients. Ann Eugen Human Genet 1933;5:30-33.

7. Block G, Patterson B, Subar A. Fruit, vegetables, and cancer prevention: a review of the epidemiological evidence. Nutr Cancer 1992;18:1-29.

8. World Health Organization \& International Agency for Research on Cancer. World Cancer Report. IARCPress, 2003.

9. Food, Nutrition and the Prevention of Cancer: a global perspective. Washington: World Cancer Research Fund \& American Institute for Cancer Research, 1997.

10. Food, Nutrition and the Prevention of Cancer: a Global Perspective. Chapter 9.1: Prevention and its benefits. World Cancer Research Fund \& American Institute for Cancer Research. Washington: BANTA Book group, 1997:538-548.

11. Brennan P, Fortes C., Butler J, Agudo A, Benhamou S, Darby S, Gerken M, Jokel KH, Kreuzer M, Mallone S, Nyberg F, Pohlabeln H, Ferro G, Bofferta P. A multicenter case-control study of diet and lung cancer among non-smokers, Cancer Causes Control 2000;11:49-58.

12. Voorrips I.E, Goldbohm RA, Verhoeven DT, van Poppel GA, Sturmans F, Hermus RJ, van den Brandt PA. Vegetable and fruit consumption and lung cancer risk in the Netherlands Cohort Study on diet and cancer. Cancer Causes Control 2000;11:101-15.

13. Diet, nutrition and the prevention of chronic diseases. World Health Organ Tech Rep Ser 2003;916:-viii, 1-149.

14. Schatzkin A, Lanza E, Corle D, Lance P, Iber F, Caan B, Shike M, Weissfeld J, Burt R, Cooper MR, Kikendall JW, Cahill J. Lack of effect of a low-fat, high-fiber diet on the recurrence of colorectal adenomas. Polyp Prevention Trial Srudy Group [see comments]. N Engl J Med 2000;342:1149-55.

15. Michels KB, Edward G, Joshipura KJ, Rosner BA, Stampfer MJ, Fuchs CS, Colditz GA, Speizer FE, Willett WC. Prospective study of fruit and vegetable consumption and incidence of colon and rectal cancers [In Process Citation]. J Natl Cancer Inst 2000;92:1740-52

16. Voorrips LE, Goldbohm RA, van Poppel G, Sturmans F, Hermus RJ, van den Brandt PA. Vegetable and fruit consumption and risks of colon and rectal cancer in a prospective cohort study: The Netherlands Cohort Study on Diet and Cancer.[In Process Citation]. Am J Epidemiol 2000;152:1081-92.

17. Key TJ. Allen NE, Spencer EA, Travis RC. Nutrition and breast cancer. Breast 2003;12:412-6. 
18. Feskanich D, Ziegler RG, Michaud DS, Giovannucci EI, Speizer FE, Willett WC, Colditz GA. Prospective study of fruit and vegetable consumption and risk of lung cancer among men and women. J Natl Cancer Inst 2000,92:1812-23.

19. Smith-Wamer SA, Spiegelman D, Yaun SS, Adami HO, Beeson WL, van den Brandt PA, Folsom AR, Fraser GE, Freudenheim II, Goldbohm RA, Graham S, Miller AB, Potter JD, Rohan TE, Speizer FE, Toniolo P, Willett WC, Wolk A, Zeleniuch-Jacquotte A, Hunter DJ. Intake of fruits and vegetables and risk of breast cancer: a pooled analysis of cohort studies. Jama 2001;285:769-76.

20. Orner G, Dashwood W, Blum C, Di, az G, Li Q. Al-Fagech M, Tebbutt N, Heath J, Emst M, Dashwood R. Response of Apc(min) and A33(DelaNbeta-cat) mutant mice to treatment with tea, sulindac, and 2-amino-1-methyl-6-phenylimidazo|4,5-blpyndine (PhIP). Mutat Res 2002;506507:121.

21. Omer GA, Dashwood WM, Blum CA, Diaz GD, Li Q. Dashwood RH. Suppression of tumorigenesis in the Apc(min) mouse: down-regulation of beta-catenin signaling by a combination of tea plus sulindac, Carcinogenesis 2003;24:263-7.

Shattuck-Brandt RL, Varilck GW, Radhika A, Yang F, Washington MtK, Dullois RN. Cyclooxygenase 2 expression is increased in the stroma of colon carcinomas from II-10(-/.) mice. Gastroenterology 2000;118:337-45.

23. van Kranen HJ, van lersel PW, Rijnkels JM, Beems DB, Alink GM, van Kreịl CF. Effects of dietary fat and a vegetable-fruit mixture on the development of intestinal neoplasia in the ApeMin mouse. Carcinogenesis 1998;19:1597-601.

24. Rijken PJ. Timmer WG, van de Kooij AJ, van Benschop IM, Wiseman SA, Mejers M, Tijburg L.B. Effect of vegetable and carotenoid consumption on aberrant crypt multiplicity, a surrogate end-point marker for colorectal cancer in azoxymethane-induced rats. Carcinogenesis 1999;20:2267-72.

25. Rijnkels JM, Alink GM. Effects of a vegetables-fruit mixture on liver and colonic 1,2dimethylhydrazine-metabolizing enzyme activities in rats fed low - or high-fat diets. Cancer Lett 1998;128:171-5.

26. Wortelboer HM, de Kruif CA, van Iersel AA, Noordhoek J, Blaauboer BJ, van Bladeren PJ, Falke HE. Effects of cooked brussels sprouts on cytochrome P-450 profile and phase II enzymes in liver and small intestinal mucosa of the rat. Food Chem Toxicol 1992;30:17-27.

27. Edenharder R, Frangart J, Hager M, Hofmann P, Rauscher R. Protective effects of fruits and vegetables against in vivo clastogenicity of cyclosphosphamide or benzo|a|pyrene in mice. Food Chem Toxicol 1998;36:637-45.

28. Guyonnet D, Siess MH, Le Bon AM, Suschetet M. Modulation of phase II enzymes by organosulfur compounds from allium vegetables in rat tissues. Toxicol Appl Pharmacol 1999;154:50-8.

29. Larsen-Su S, Williams DE. Dietary indole-3-carbinol inhibits FMO activity and the expression of flavin-containing monooxygenase form 1 in rat liver and intestine. Drug Metab Dispos 1996:24:927. 31.

30. Talalay P, Fahey JW. Phytochemicals from cruciferous plants protect against cancer by modulating carcinogen metabolism. J Nutr 2001;131:3027S-33S.

31. Mahmoud NN, Carothers AM, Grunberger D, Bilinski RT, Churchill MR, Martucci C, Newmark $\mathrm{HI}$, Bertagnolli MM. Plant phenolics decrease intestinal tumors in an animal model of familial adenomatous polyposis. Carcinogenesis 2000;21:921-7.

32. Bonnesen C, Stephensen PU, Andersen O, Sorensen H, Vang O. Modulation of cytochrome P-450 and glutathione S-transferase isoform expression in vivo by intact and degraded indolyl glucosinolates. Nutr Cancer 1999;33:178-87. 
33. Katchamart S, Williams DE. Indole-3-carbinol modulation of hepatic monooxygenases CYP1A1, CYP1A2 and FMO1 in guinea pig, mouse and rabbit. Comp Biochem Physiol C Toxicol Pharmacol 2001;129:377-84.

34. Smith T], Yang CS. Effect of organosulfur compounds from garlic and cruciferous vegetables on drug metabolism enzymex. Drug Metabol Drug Interact 2000;17:23-49.

35. Deschner EE, Ruperto J, Wong G, Newmark HL Quercetin and rutin as inhibitors of azoxymethanol-induced colonic neoplasia. Carcinogenesis 1991;12:1193-6.

36. Beiman S. Onion and garlic oils inhibit tumor promotion. Carcinogenesis 1983;4:1063-5.

37. Sorensen M, Jensen BR, Poulsen HE, Deng X, Tygstrup N, Dalhoff K, Loft S. Effects of a Brussels sprouts extract on oxidative DNA damage and metabolising enzymes in rat liver. Food Chem Toxicol 2001;39:533-40.

38. Kallay E, Adlercreutz H, Farhan H, Lechner D, Bajna E, Gerdenitsch W, Campbell M, Cross HS. Phytoestrogens regulate vitamin D metabolism in the mouse colon: relevance for colon tumor prevention and therapy. J Nutr 2002;132:3490S-3493S.

39. Vang $\mathrm{O}$, Jensen $\mathrm{H}$, Autrup $\mathrm{H}$. Induction of cytochrome P-450IA1, IA2, IIB1, IIB2 and IIE1 by broccoli in rat liver and colon. Chem Biol Interact 1991;78:85-96.

40. Zeng H, Davis CD, Finley JW. Effect of selenium-enriched broccoli diet on differential gene expression in min mouse liver(1,2). J Nutr Biochem 2003;14:227-231.

41. Kim DJ, Shin DH, Ahn B, Kang JS, Nam KT, Park CB, Kim CK, Hong JT, Kim YB, Yun YW, Jang DD, Yang KH. Chemoprevention of colon cancer by Korean food plant components. Mutat Res 2003:523-524:99-107.

42. Kararli TT. Comparison of the gastrointestinal anatomy, physiology, and biochemistry of humans and commonly used laboratory animals. Biopharm Drug Dispos 1995;16:351-80.

43. Potter JD. Cancer prevention: epidemiology and experiment. Cancer lett 1997;114:7-9.

44. Greenwald P. Clifford CK, Milner JA. Diet and cancer prevention, Eur J Cancer 2001;37;948-65.

45. Steinmetz KA, Potter JD. Vegetables, fruit, and cancer. II, Mechanisms. Cancer Causes Control 1991:2:427-42.

46. Temple NJ, Kaiser Gladwin K. Fruit, vegetables, and the prevention of cancer. Research challenges. Nutrition 2003;19:467-70.

47. Manson MM, Geseher A, Hudson E.A, Plummer SM, Squires MS, Prigent SA. Blocking and suppressing mechanisms of chemoprevention by dietary constituents. Toxicol Lett 2000;112113:499-505.

48. Stecle VE, Boone CW, Lubet RA, Crowell JA, Hoimes CA, Sigman CC. Kelloff G]. Preclinical drug development paradigms for chemopreventives. Hematol Oncol Clin North Am 1998;12:943-61, v-vi.

49. De Flora S. Mechanisms of inhibitors of mutagenesis and carcinogenesis. Mutat Res 1998;402:151-8.

50. Kelloff G], Crowell JA, Stecle VE, Lubet RA, Boone CW, Malone WA, Hawk ET, Lieberman R, Lawrence JA, Kopelovich L, Als I, Viner JL, Sigman CC. Progress in cancer chemoprevention. Ann N Y Acad Sci 1999;889:1-13.

51. Dragsted LO, Strube M, Lansen JC. Cancer-protective factors in fruits and vegetables: biochemical and biological background. Pharmacol Toxicol 1993;72:116-35.

52. Wattenberg LW. Inhibition of carcinogenesis by minor dietary constituents. Cancer Res 1992:52:2085s-2091s,

53. Jacobs LR. Fiber and colon cancer. Gastroenterol Clin North Am 1988:17:747-60.

54. Bartsch H. Ohshima H. Pignatelli B. Inhibitors of endogenous nitrosation. Mechanisms and implications in human cancer prevention. Mutat Res 1988:202:307-24. 
55. Lampe JW, Peterson S. Brassica, biotransformation and cancer risk: genetic polymorphisms alter the preventive effects of cruciferous vegetables. J Nutr 2002;132-2991-4.

56. van Poppel G, Vethoeven DT, Verhagen H, Goldbohm RA. Brassica vegetables and cancer prevention. Epidemiology and mechanisms. Adv Exp Med Biol 1999;472:159-68.

57. Nijveldt RJ, van Nood E, van Hoom DE, Boelens PG, van Norren K, van Lecuwen PA. Flavonoids: a review of probable mechanisms of action and potential applications. Am J Clin Nutr 2001:74:418-25.

58. Ross JA, Kasum CM. Dictary flavonoids: bioavailability, metabolic effects, and safery. Annu Rev Nutr 2002:22:19-34.

59. Bianchini $\mathrm{F}$, Vainio $\mathrm{H}$. Allium vegetables and organosulfur compounds: do they help prevent cancert? Environ Health Perspect 2001:109:893-902.

60. Verhoeven DT, Verhagen H, Goldbohm RA, van den Brandt PA, van Poppel G. A review of mechanisms underlying anticarcinogenicity by brassica vegetables. Chem Biol Interact 1997;103:79. 129.

61. Murillo G, Mehta RG. Cruciferous vegetables and cancer prevention. Nutr Cancer 2001;41:17-28.

62. Awasthi YC, Singhal SS, Awasthi S. Mechanisms of anticarcinogenic effects of antioxidant nutrients. In: Watson RR, Mufti SI, eds. Nutrition and Cancer Prevention. Boca Raton, Florida: CRC Press, Inc., 1996:139-172.

63. Collins AR, Olmedilla B, Southon S, Granado F, Duthie SJ. Serum carotenoids and oxidative DNA damage in human lymphocytes. Carcinogenesis 1998;19:2159-62.

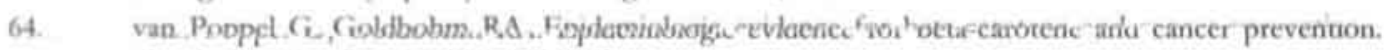
Am J Clin Nutr 1995;62:1393S-1402S.

65. De Flora S, Zanacchi P, Izotti H, Hayatsu H. Mechanisms of food-borne inhibitors of genotoxicity relevant to cancer prevention. In: Hayatsu H, ed. Mutagens in Food. Detection and Prevention. Boca Raton: CRC Press, 1991:157-180.

66. Dutta $\Lambda$, Dutta SK. Vitamin $\mathrm{E}$ and its role in the prevention of atherosclerosis and carcinogenesis: a review. J Am Coll Nutr 2003;22:258-68.

67. Troll W, Wiesner R, Frenkel K. Anticarcinogenic action of protease inhibitors. Adv Cancer Res 1987:49:265-83.

68. Gamet-Payrastre L, Li P, Lumeau S, Cassar G, Dupont MA, Chevolleau S, Gasc N, Tulliez J. Terce F. Sulforaphane, a naturally occurring isothiocyanate, induces cell cycle arrest and apoptosis in HT29 - human colon cancer cells. Cancer Res 2000;60:1426-33.

69. Johnson IT. Anticarcinogenic effects of diet-related apoptosis in the colorectal mucosa. Food Chem Toxicol 2002;40:1171-8.

70. Adams J. Proteasome inhibition: a novel approach to cancer therapy. Trends Mol Med 2002;8:S4954.

71. Frommel TO, Mobarhan S, Doria M, Halline AG, Luk GD, Bowen PE, Candel A, Liao Y. Effect of beta-carotene supplementation on indices of colonic cell proliferation. J Natl Cancer Inst 1995;87:1781-7.

72. Palozza P, Serini S, Maggiano N, Angelini M, Boninsegna A, Di Nicuolo F, Ranelletti FO, Calviello G. Induction of cell cycle arrest and apoptosis in human colon adenocarcinoma cell lines by betacarotene through down-regulation of cyclin A and Bcl-2 family proteins. Carcinogenesis 2002;23:118 . 
73. Phillips RW, Kikendall JW, Luk GD, Willis SM, Murphy JR, Maydonovitch C, Bowen PE, Stacewicz-Sapuntzakis M, Wong RK. beta-Carotene inhibits rectal mucosal omithine decarboxylase activity in colon cancer patients. Cancer Res 1993;53:3723-5.

74. Cover CM, Hsieh SJ, Tran SH, Hallden G, Kim GS, Bjeldanes LF, Firestone GL, Indole-3-carbinol inhibits the expression of cyclin-dependent kinase-6 and induces a G1 cell cycle arrest of human breast cancer cells independent of estrogen receptor signaling. J Biol Chem 1998;273:3838-47.

75. Wenzel U, Kuntz S, Brendel MD, Daniel $\mathrm{H}$. Dietary flavone is a potent apoptosis inducer in human colon carcinoma cells, Cancer Res 2000;60:3823-31.

76. Bonnesen C, Eggleston IM, Hayes JD. Dietary indoles and isothiocyanates that are generated from cruciferous vegetables can both stimulate apoptosis and confer protection against DNA damage in human colon cell lines. Cancer Res 2001;61:6120-30.

77. MeBurney MW, Costa S, Pratt MA. Retinoids and cancer a basis for differentiation therapy. Cancer Invest 1993;11:590-8.

78. Ren W, Qiao Z, Wang H, Zhu L, Zhang L. Flavonoids; promising anticancer agents. Med Res Rev 2003;23:519-34.

79. Honma Y, Okabe-Kado J, Kasukabe T, Hozumi M, Umezawa K. Inhibition of abl oncogene tyrosine kinase induces erythroid differentiation of human myelogenous leukemia K562 cells. Jpn J Cancer Res 1990;81:1132-6.

80. Bertram JS, Carotenoids and gene regulation. Nutr Rev 1999:57:182-91.

81. Bertram JS, Bortkiewicz H. Dietary carotenoids inhibit neoplastic transformation and modulate gene expression in mouse and human cells. Am J Clin Nutr 1995;62:1327S-1336S.

82. Schelp FP, Pongpaew P. Protection against cancer through nutritionally-induced increase of endogenous proteinase inhibitors-a hypothesis. Int J Epidemiol 1988;17:287-92.

83. Meydani SN, Meydani M, Blumberg JB, Leka LS, Siber G, Loszewski R, Thompson C, Pedrosa MC, Diamond RD, Stollar BD. Vitamin E supplementation and in vivo immune response in healthy elderiy subjects. A randomized controlled trial. Jama 1997;277:1380-6.

84. Siegel BV. Enhancement of interferon production by poly $(\mathrm{rl})$-poly $(\mathrm{rC})$ in mouse cell cultures by ascorbic acid. Nature 1975;254:531-2.

85. Reifen R. Vitamin A as an anti-inflammatory agent. Proc Nutr Soc 2002;61:397-400.

86. Hughes DA. Effects of carotenoids on human immune function. Proc Nutr Soc 1999;58:713-8.

87. Michnovicz II. Bradlow HL. Ntered estrogen metabolism and excretion in humans following consumption of indole-3-carbinol. Nutr Cancer 1991;16:59-66.

88. Messina M, Barnes S. The role of soy products in reducing risk of cancer. I Natl Cancer Inst 1991;83:541-6.

89. Tosetti F, Ferran N, De Flora S, Albini A. Angioprevention': angiogenesis is a common and key tanget for cancer chemopreventive agents. Faseb J 2002;16:2-14.

90. Meng Q. Qi M, Chen DZ, Yuan R, Goldberg ID, Rosen EM, Auborn K, Fan S. Suppression of breast cancer invasion and migration by indole-3-carbinol: associated with up-regulation of BRCA1 and E-cadherin/catenin complexes. J Mol Med 2000;78:155-65.

91. Gartner 1.P. Hiatt 11_ Chapter 17; Digestive System: Alimentary Canal. Color textbook of histology. second edition. Philadelphia: W.B. Saunders Company, 2001:379-409.

92. Troisi RJ, Freedman AN, Devesa SS. Incidence of colorectal carcinoma in the U.S.: an update of trends by gender, race, age, subsite, and stage, 1975-1994. Cancer 1999;85:1670-6.

93. Parkin DM. Epidemiology of cancer: global patterns and trends. Toxicol Lett 1998;102-103:227-34.

94. Potter JD, Colorectal cancer molecules and populations. J Natl Cancer Inst 1999;91:916-32. 
95. Food, Nutrition and the Prevention of Cancen a Global Perspective. Chapter 4.10. Colon, rectum. World Cancer Research Fund \& American Institute for Cancer Research. Washington: BANTA Book group, 1997:216-251.

96. Bruce WR, Giacca A, Medline A. Possible mechanisms relating diet and risk of colon cancer. Cancer Epidemiol Biomarkers Prev 2000;9:1271-9.

97. Doll R, Peto R. The causes of cancer quantitative estimates of avoidable risks of cancer in the United States today. J Nati Cancer Inst 1981;66:1191-308.

98. Willett WC. Diet, nutrition, and avoidable cancer. Einviron Health Perspect 1995;103 Suppl 8:165-70.

99. van't Veer P. Jansen MC, Klerk M, Kok F. Fruits and vegetables in the prevention of cancer and candiovascular disease. Public Health Nutr 2000;3:103-7.

100. Lynch HT, de la Chapelle A. Hereditary colorectal cancer. N Engl J Med 2003:348:919-32.

101. Syngal S, Clarke G, Bandipalliam P. Potential roles of genetic biomarkers in colorectal cancer chemoprevention. J Cell Biochem Suppl 2000;34:28-34.

102. Levin B. Genetic syndromes as potential targets for chemoprevention of colorectal neoplasia. J Cell Biochem Suppl 2000;34:19-22.

103. Fearon ER, Jones PA. Progressing towand a molecular description of colorectal cancer development. Faseb J 1992:6:2783-90.

104. Fearon ER, Vogelstein B. A genetic model for colorectal tumorigenesis. Cell 1990,61:759-67.

105. Fujiwara T, Stolker JM, Watanabe T, Rashid A, Longo P, Fishleman JR, Booker S, Lynch HT, Jass JR, Green JS, Kim H, Jen J. Vogelstein B, Hamilton SR. Accumulated clonal genetic alterations in familial and sporadic colorectal carcinomas with widespread instability in microsatellite sequences. Am J Pathol 1998;153:1063-78.

106. Grady WM, Markowitz SD. Genetic and epigenetic alterations in colon cancer. Annu Rev Genomics Hum Genet 2002;3:101-28.

107. Ilyas M, Straub J, Tomlinson IP, Bodmer WF. Generic pathways in colorectal and other cancers. Eur J Cancer 1999;35:335-51.

108. Chung DC. The genetic basis of colorectal cancer: insights into critical pathways of tumorigenesis. Gastroenterology 2000;119:854-65.

109. Arends JW. Molecular interactions in the Vogelstein model of colorectal carcinoma. J Pathol 2000; 190:412-6.

110. Hu R, Kim BR, Chen C, Hebbar V, Kong AN. The roles of JNK and apoptotic signaling pathways in PEITC-mediated responses in human HT-29 colon adenocarcinoma cells. Carcinogenesis 2003:24:1361-7.

111. Clapper ML, Szarka CE, Pfeiffer GR, Graham TA, Balshem AM, Litwin S, Goosenberg EB, Frucht $\mathrm{H}$, Engstrom PF. Preclinical and clinical evaluation of broccoli supplements as inducers of glutathione S-transferase activity. Clin Cancer Res 1997;3:25-30.

112. Kallay E, Adlerereutz H, Farhan H, Lechner D, Bajna E, Gerdenitsch W, Campbell M, Cross HS. Phytoestrogens Regulate Vitamin D Metabolism in the Mouse Colon: Relevance for Colon Tumor Prevention and Therapy. J Nutr 2002;132:3490S-3493S.

113. Sparnins VL, Venegas PL, Wattenberg I.W. Glutathione S-transferase activity: enhancement by compounds inhibiting chemical carcinogenesis and by dietary constituents. I Natl Cancer Inst 1982;68:493-6.

114. Mori H, Tanaka T, Sugie S, Yoshimi N, Kawamori T, Hirose Y, Ohnishi M. Chemoprevention by naturally occurring and synthetic agents in oral, liver, and large bowel carcinogenesis. J Cell Biochem Suppl 1997;27:35-41. 
115. Kato T, Hancock R, Mohammadpour H, McGregor B, Manalo P, Khaiboullina S, Hall M, Pardini L, Pardini R. Influence of omega- 3 fatty acids on the growth of human colon carcinoma in nude mice. Cancer Lett 2002;187:169.

116. Nijhoff WA, Grubben MJ, Nagengast FM, Jansen JB, Verhagen H, van Poppel G, Peters WH. Effects of consumption of Brussels sprouts on intestinal and lymphocytic glutathione S-transferases in humans. Carcinogenesis 1995;16:2125-8.

117. Persson I, He I, Fang C, Normen I, Rylander R. Influence of vegetables on the expression of GSTP1 in humans--a pilot intervention study (Sweden). Cancer Causes Control 2000;11:359-61.

118. Lampe JW, King IB, Li S, Grate MT, Barale KV, Chen C, Feng Z, Potter JD. Brassica vegetables increase and apiaceous vegetables decrease cytochrome P450 $1 \mathrm{~A} 2$ activity in humans: changes in caffeine metabolite tatios in response to controlled vegetable diets. Carcinogenesis 2000;21:1157-62.

119. Kall MA, Vang O, Clausen J. Effects of dietary broccoli on human in vivo drug metabolizing enzymes: evaluation of caffeine, oestrone and chlorzoxazone metabolism [see comments]. Carcinogenesis 1996;17:793-9.

120. Pantuck EJ, Pantuck CB, Anderson KE, Wattenberg LW, Conney AH, Kappas A. Effect of brussels sprouts and cabbage on drug conjugation. Clin Pharmacol Ther 1984:35:161-9.

121. Faivre J, Couillault C, Belghiti C. Intervention trials on colorectal cancer prevention. IARC Sci Publ 1996:115-24.

122. Maclennan R, Macrae F, Bain C, Battistutta D, Chapuis P, Gratten H, Lambert J, Newland RC, Ngu M, Russell A, et al. Randomized trial of intake of fat, fiber, and beta carotene to prevent colorectal adenomas. The Australian Polyp Prevention Project [see comments]. I Nat Cancer Inst 1995;87:1760-6.

123. Gartner LP, Hiatt JL. Chapter 15; Respiratory System. Color textbook of histology, second edition. Philadelphia: W.B. Saunders Company, 2001:343-364.

124. Food, Nutrition and the Prevention of Cancer: a Global Perspective. Chapter 4.5: Lung. World Cancer Research Fund \& American Institute for Cancer Research. Washington: BANTA Book group, 1997:130-147.

125. Alberg AJ, Samet JM. Epidemiology of lung cancer. Chest 2003;123:21S-49S.

126. Omenn GS. Chemoprevention of lung cancer, the rise and demise of beta-carotene. Annu Rev Public Health 1998;19:73-99.

127. Ziegler RG, Mayne ST, Swanson CA. Nutrition and lung cancer. Cancer Causes Control 1996;7:15777.

128. Fong KM, Minna JD. Molecular biology of lung cancer: clinical implications. Clin Chest Med 2002;23:83-101.

129. You M, Bergman G. Preclinical and clinical models of lung cancer chemoprevention. Hematol Oncol Clin North Am 1998;12:1037-53.

130. Szabo E, Mulshine J. Epidemiology, prognostic factors, and prevention of lung cancer. Curr Opin Oncol 1993;5:302-9.

131. Ooi WL, Elston RC, Chen VW, Bailey-Wilson JE, Rothschild H. Increased familial risk for lung cancer. J Natl Cancer Inst 1986;76:217-22.

132. Sellers TA, Bailey-Wilson JE, Elston RC, Wilson AF, Elston GZ, Ooi WL, Rothschild H. Evidence for mendelian inheritance in the pathogenesis of lung cancer.J Nati Cancer Inst 1990;82:1272-9.

133. Vineis P, Malats N, Lang A, d'Errico A, Caporaso N, Cuzick J, Boffetta P. Metabolic polymorphisms and susceptibility to cancer. IARC Scientific Publications No.148, 1999. 
134. Spivack SD, Faseo MJ. Walker VE, Kaminsky LS. The moleculat epidemiology of lung cancer. Crit Rev Toxicol 1997:27:319-65.

135. Zochbauer-Muller S, Gazdar AF, Minna JD. Molecular pathogenesis of lung cancer. Annu Rev Physiol 2002;64:681-708.

136. Sekido Y, Fong KM, Minna JD. Molecular genetics of lung cancer. Annu Rev Med 2003;54:73-87,

137. Sanchez-Cespedes M. Dissecting the genetic alterations involved in lung carcinogenesis, Lung Cancer 2003,40:111-21.

138. Bai F, Matsui T, Ohtani-Fujita N, Matsukawa Y, Ding Y, Sakai T. Promoter activation and following induction of the p21/WAF1 gene by flavone is involved in 61 phase arrest in A549 lung adenocarcinoma cells. FEBS Lett 1998;437:61-4.

139. Banoub RW, Femstrom M, Ruch RJ. Lack of growth inhibition or enhancement of gap junctional intercellular communication and connexin 43 expression by beta-carotene in murine lung cpithelial cells in vitro, Cancer Lett 1996;108:35-40.

140. Paolini M, Perocco P, Canistro D, Valgimigli I, Pedulli GF, Ioni R, Croce CD, Cantelli-Forti G, Legator MS, Abdel-Rahman SZ. Induction of cytochrome P450, generation of oxidative stress and in vitro cell-transforming and DNA-damaging activities by glucotaphanin, the bioprecursot of the chemopreventive agent sulforaphane found in broccoli. Carcinogenesis 2004:25:61-7.

141. Yano T, Yano Y, Yajima S, Kumadaki I, Ichikawa T, Otani S, Hagiwara K. The suppression of ornithine decarboxylase expression and cell proliferation at the promotion stage of lung tumorigenesis in mice by alpha-tocopheryloxybutyric acid. Biochem Pharmacol 2001,61:1177-81.

142. Khanduja KL, Gandhi RK, Pathaniai V, Syal N. Prevention of $\mathrm{N}$-nitrosodiethylamine-induced lung tumorigenesis by ellagic acid and quercetin in mice. Food Chem Toxicol 1999;37:313-8.

143. Chung FL, Morse MA, Eklind KI, Xu Y. Inhibition of tobacco-specific nitrosamine-induced lung rumorigenesis by compounds derived from cruciferous vegetables and green tea. Ann N Y Acad Sci 1993;686:186-201; discussion 201-2.

144. Chung FL, Kelloff G, Steele V, Pittman B, Zang E, Jiao D, Rigotty J, Choi CI, Rivenson A. Chemopreventive efficacy of arylalkyl isothiocyanates and $\mathrm{N}$-acetyleysteine for lung rumorigenesis in Fischer rats. Cancer Res 1996;56:772-8.

145. Omenn GS, Goodman G, Thornquist M, Grizzle J, Rosenstock I, Barnhart S, Balmes J, Cherniack MG, Cullen MR, Glass A, et al. The beta-carotene and retinol efficacy trial (CARET) for chemoprevention of lung cancer in high risk populations: smokers and asbestos-exposed workers.

- Cancer Res 1994;54:2038s-2043s.

146. The effect of vitamin $\mathrm{E}$ and beta carotene on the incidence of lung cancer and other cancers in male smokers. The Alpha-Tocopherol, Beta Carotene Cancer Prevention Study Group. N Engl J Med 1994:330:1029-35.

147. Kelley MJ, McCrory DC. Prevention of lung cancer: summary of published evidence. Chest 2003;123:50S-59S.

148. Thomas PS. Hybridization of denatured RNA and small DNA fragments transferred to nitrocellulose. Proc Natl Acad Sci U S A 1980;77:5201-5.

149. Liang P, Pardec AB. Differential display of eukaryotic messenger RNA by means of the polymerase chain reaction. Science 1992;257:967-71.

150. Bustin SA. Absolute quantification of mRNA using real-time reverse transcription polymerase chain reaction assays. J Mol Endocrinol 2000;25:169-93.

151. Bustin SA. Quantification of mRNA using real-time reverse transcription PCR (RT-PCR); trends and problems. J Mol Endocrinol 2002;29:23-39. 
152. Veiculescu VE, Zhang L, Vogelstein B, Kinzler KW. Serial analysis of gene expression. Science 1995;270:484-7.

153. Schena M, Shalon D, Davis RW, Brown PO, Quantitative monitoring of gene expression patterns with a complementary DNA microarray [see comments]. Science 1995:270:467-70.

154. Ball CA, Sherlock G, Parkinson H, Rocea-Sera P, Brooksbank C, Causton HC, Cavalieri D, Gaasterland T, Hingamp P, Holstege F, Ringwald M, Spellman P, Stoeckert CJ, Jr., Stewart JE, Taylor R, Brazma A, Quackenbush J. Standards for microarray data. Science 2002;298:539.

155. Butte A. The use and analysis of microarray data. Nat Rev Drug Discov 2002;1:951-60.

156. Cheung VG, Morley M, Aguilar F, Massimi A, Kucherlapati R, Childs G. Making and reading microarrays. Nat Genet 1999:21:15-9.

157. Debouck C, Goodfellow PN. DNA microarrays in drug discovery and development. Nat Genet 1999:21:48-50,

158. Habeck M. New approach to gene expression analysis. Drug Discov Today 2003;8:427-8.

159. Holloway AJ, Van Laar RK, Tothill RW, Bowtell DD. Options available-from start to finish-for obtaining data from DNA microarrays II. Nat Genet 2002;32 Suppl 2:481-9.

160. Kerr MK, Churchill GA. Statistical design and the analysis of gene expression microarray data. Genet Res 2001;77:123-8,

161. Lemieux B, Aharoni A, Schena M. Overview of DNA chip technology. Molecular Breeding 1998:4:277.89.

162. Lee CK, Klopp RG, Weindruch R, Prolla TA. Gene expression profile of aging and its retardation by caloric restriction |see comments]. Science 1999:285:1390-3.

163. Sreckumar R, Unnikrishnan J, Fu A, Nygren J, Short KR, Schimke J, Barazzoni R, Nair KS. Impact of high-fat diet and antioxidant supplement on mitochondrial functions and gene transcripts in rat muscle. Am J Physiol Endocrinol Metab 2002;282:E1055-61.

164. Chen CC, Shich B, Jin YT, Liau YE, Huang CH, Liou JT, Wu LW, Huang W, Young KC, Lai MD, Liu HS, Li C. Microarray profiling of gene expression patterns in bladder tumor cells treated with genistein. J Biomed Sci 2001;8:214-22.

165. Nadler ST, Attic AD. Please pass the chips: genomic insights into obesity and diabetes. J Nutr 2001:131:2078-81.

166. Kaput J, Rodriguez RI. Nutritional genomics: the next frontier in the postgenomic era. Physiol Genomics 2004:16:166-77.

167. Muller M, Kersten S. Opinion: Nutrigenomics: goals and strategies. Nat Rev Genet 2003;4:315-22

168. Russo G, Zegar C, Giordano A. Advantages and limitations of microarray technology in human cancer. Oncogene 2003;22:6497-507.

169. Cunningham MJ. Genomics and proteomics; the new millennium of drug discovery and development. J Pharmacol Toxicol Methods 2000;44:291-300.

170. Sydor JR, Nock S. Protein expression profiling arrays: tools for the multiplexed high-throughput analysis of proteins, Proteome Sci 2003:1:3.

171. Lockhart DJ, Winzeler EA. Genomics, gene expression and DNA arrays. Narure 2000,405:827-36.

172. Murphy D. Gene expression studies using microarrays: principles, problems, and prospects. Adv Physiol Educ 2002;26:256-70.

173. Barrett JC, Kawasaki ES. Microarrays: the use of oligonucleotides and CDNA for the analysis of gene expression. Drug Discov Today 2003;8:134 41.

174. Goldsmith ZG, Dhanasekaran N. The microtevolution: applications and impacts of microarray technology on molecular biology and medicine (review). Int J Mol Med 2004;13:483-95. 




\title{
Chapter 2
}

\section{Altered vegetable intake affects pivotal carcinogenesis pathways in colon mucosa from adenoma patients and controls}

Accepted for publication in Carcinogenesis

\author{
S.G.J. van Breda' \\ E. van Agen' \\ L.G.J.B. Engels 2 \\ E.J.C. Moonen' \\ J.C.S. Kleinjans ${ }^{1}$ \\ J.H.M. van Delft ${ }^{1}$
}

'Department of Health Risk Analysis and Toxicology, Maastricht University, Maastricht, The Netherlands 2Department of Gastroenterology, Masland Hospital Sittard, Sirtard, The Netherlands 


\section{Abstract}

The evidence from epidemiological and experimental studies that vegetables reduce the risk of colorectal cancer is convincing. However, the involved genes and genetic pathways are not clear. The aim of this study was to identify genes that are modulated in vivo in colorectal mucosa by vegetables, and to investigate whether colon adenoma patients respond differently compared to healthy controls. Twenty female adenoma patients and eight healthy controls were randomly split into two groups of ten and four persons respectively, receiving either a $50 \%$ decreased $(=75 \mathrm{~g} /$ day $)$ or doubled $(=300 \mathrm{~g} /$ day) intake of vegetables for two weeks. In order to assess effects on gene expression at target level, colorectal biopsies were collected before and after the intervention. Total RNA was isolated from the biopsies to measure gene expression of 597 genes relevant for responses to xenobiotics by microarray technology, followed by confidence analyses to identify differentially expressed genes. Mainly genes related to cell cycle control and genes for oxidoreductase activities were over-represented in the list of modulated genes. Twenty genes were modulated which are known to be related to (colon)carcinogenesis. Seven genes were similarly modulated in patients and controls, for example fos proto-oncogene and ornithine decarboxylase. Thirteen genes were modulated differently in patients compared to controls, including cyclooxygenase-2 and Human mdm2-A in patients and cytochrome P450-27B1, $-2 \mathrm{C} 19,-2 \mathrm{D} 6,-2 \mathrm{C} 9$, and $-3 \mathrm{~A} 4$ in controls. Almost all the effects on modulating the expression of genes by altering vegetable intake can be mechanistically linked to cellular processes that explain either prevention of colorectal cancer risk by high vegetable intake or increased colorectal cancer risk by low vegetable intake. Furthermore, it seems that vegetables in patients affect genes involved in late stage of colorectal cancer, whereas in controls genes involved in the initiation phase are modulated. 


\section{Introduction}

Worldwide, colorectal cancer (CRC) is one of the most common cancers. Approximately 943,000 new cases were diagnosed and 491,000 people died of CRC in the year 2000.1 Although early detection of CRC is important, prevention has more potential for control of cancer. ${ }^{2}$

The predominantly modifiable determinant of CRC is the diet. The most consistent finding is the association of vegetables with reduced risk of CRC. ${ }^{14}$ Numerous potentially anticarcinogenic agents are present in vegetables which have various potential mechanisms of action in the initiation and later stages of carcinogenesis. Compounds classified as blocking agents can prevent, or greatly reduce, initiation of carcinogenesis by altering the profile of both phase I and II drug metabolizing enzymes, by scavenging reactive oxygen and other free radical species and by altering rates of DNA repair. Suppressing agents affect later stages of carcinogenesis by reducing cell proliferation. This involves modulation of signal transduction pathways, leading to altered gene expression, cellcycle arrest or apoptosis and thereby preventing the accumulation of damaged cells, 2,5-7

A genetic model for CRC has been presented by Fearon and Vogelstein ${ }^{8}$ which has been subsequently extended with additional genetic events and specific molecular pathways ${ }^{9}$ presenting a network of molecular genetic pathways. ${ }^{810}$ These genetic pathways and the involved genes are obvious molecular targets for the protection against CRC by vegetables. Nowadays available microarray technology makes it feasible to assess the effect of a specific diet on the expression of multiple genes simultaneously. "I

The aim of this study was to identify genes that are modulated in vivo in colorectal mucosa by vegetables, and to investigate whether colon adenoma patients respond differently in comparison to healthy controls. Therefore, a human dietary intervention study was carried out in which the effect of an increased or decreased intake of vegetables on gene expression was investigated in biopsies from normal colorectal mucosa of female sporadic adenoma patients and healthy controls by applying microarray technology. A descriptive evaluation was made of the gene expression results with respect to their possible role in mechanisms underlying CRC risk.

\section{Materials and Methods}

\section{Subjects and Study Design}

The study population was recruited at the Maasland Hospital Sittard, the Netherlands. Twenty female patients (mean age 61.5 \pm 7.7 years, range $53-78$ years) and eight healthy female controls (mean age $48.1 \pm 10.2$ years, range 33-65 years) were selected from the 
colonoscopy control schedule. Eligible patients had a history of colorectal adenomas but no familial CRC syndrome or inflammatory bowel disease; eligible controls had no history of colorectal adenomas or other colorectal disorders. No colorectal mucosal diseases were permitted in patients as well as in controls. Existing adenomas were endoscopically removed during the examination. These two groups were chosen to investigate whether the effect of the intervention is different for patients, compared to controls. It is plausible that vegetables exert a different effect in persons who are at higher risk for the development of CRC.

Both patients and controls were randomly split into two groups of ten and four persons respectively, receiving either a low $(75 \mathrm{~g} /$ day $)$ or high $(300 \mathrm{~g} /$ day $)$ vegetable diet for a period of two weeks. The length of this intervention period was chosen based on results of other studies, in which the effect of a vegetable intervention on gene expression changes was examined in various tissues including lymphocytes and colorectal mucosa. In these studies, the length of intervention was equal or even shorter than two weeks and still a modulation of gene expression (mostly biotransformation genes) was reported. ${ }^{12-16} \mathrm{Next}$, taking into account that the mean intake of vegetables of adult females in the Netherlands is approximately $150 \mathrm{~g} /$ day $^{17}$, this implies that, on average, subjects in the low vegetable group decreased their vegetable intake by $50 \%$ and subjects in the high vegetable group doubled their vegetable intake. A frozen vegetable mix was provided, consisting of $30 \%$ wt/wt cauliflower, $30 \% \mathrm{wt} / \mathrm{wt}$ peas, $30 \% \mathrm{wt} / \mathrm{wt}$ carrots and $10 \% \mathrm{wt} / \mathrm{wt}$ onions (Findus, Sweden) together with instructions for preparation. During the intervention period, subjects followed their normal dietary habits with, however, adapting their vegetable consumption in that sense that they were allowed to consume only the provided vegetables.

Four rectal biopsies and $20 \mathrm{ml}$ peripheral blood were sampled at the first endoscopic examination and at the day following the last day of the intervention period. Biopsies were taken from healthy mucosal tissue of the rectum $10 \mathrm{~cm}$ above the level of the anus, immediately frozen in liquid nitrogen and stored at $-80^{\circ} \mathrm{C}$ until use. The rectum and colon are regarded as one anatomical and functional entity and therefore rectal biopsies represent colonic environment. ${ }^{18}$ Aliquots of blood plasma were stored at $-20{ }^{\circ} \mathrm{C}$ until analyses. In order to assess the compliance with the intervention, levels of $\alpha$ - and $\beta$ carotene, retinol and $\alpha$-tocopherol in plasma were determined by the method of Hess et $\mathrm{al}^{19}$ by HPLC-UV. By means of questionnaires, the dietary and life-style habits on two consecutive days before and during the intervention period were recorded. Energy and nutrient intake were calculated using the computerized Dutch Nutrient Data bank. ${ }^{20}$

This study was approved by the Medical Ethical Committee of the Maasland Hospital in Sittard and written informed consent was obtained from the participants prior to the start of the study. 


\section{Total RNA Isolation and dDNA Probe Synthesis}

Four biopsies (ca. $20 \mathrm{mg}$ in total) from each subject were pooled and ground to a powder in a stainless steel mortar under liquid nitrogen and homogenized in $800 \mu \mathrm{l}$ TRIZOL. Reagent (Gibco/BRL). Total RNA was extracted according to the manufacturer's instructions. The RNeasy@ Mini Kit (Qiagen) was used to purify total RNA from salts and residual DNA. Quantity of each RNA sample was measured by a spectrophotometer and varied from 25 to $50 \mu \mathrm{g}$. Integrity was determined by a Bioanalyzer (Agilent Technologies Netherlands B.V., The Netherlands). All samples contained intact total RNA with an rRNA ratio $(28 \mathrm{~S} / 18 \mathrm{~S})>1.5$.

Cyanine 3- (Cy3) and Cyanine 5- (Cy5) labeled cDNA probes were prepared using $10 \mu \mathrm{g}$ total RNA from each subject before and after the intervention period, respectively, by the method of Hasseman et al. ${ }^{21}$ Each subject acted as its own control, meaning that the fluorescent-labeled cDNAs of each subject before and after the intervention were mixed. A second microarray experiment was carried out for each subject whereby the dyes were switched (flip-dye experiment).

\section{Micmamay Experiments}

Gene expression analysis was on the PHASE-1 Microarray Human-600 (PHASE-1 Molecular Toxicology, Santa fee, USA), which contain 597 sequence verified human genes, representing biologically relevant and control genes. Each array batch passed the slide quality index in which several parameters such as signal to noise ratios, COVs, laser intensities, and others were taken into account. PHASE-1 has routinely confirmed their microarrays by using RT-PCR, histological analysis and other accurate measures of expression. The genes were selected based on relevance for responses of cells to xenobiotic compounds and knowledge of the involved processes. The presented pathways relate to inflammation, DNA damage and repair, oxidative stress, cell signaling, cell proliferation, detoxification, metabolism, transcription and apoptosis, which al relate to the genetic model of CRC. ${ }^{8-10}$ Target genes are single stranded, approximately 500 nucleotides in length and spotted in quadruplicate on glass microscope slides.

Labeled cDNA probe of each subject was hybridized to the PHASE-1 microarray according to the manufacturer's instructions. Slides were scanned on a GMS 418 Array Scanner (Affymetrix, High Wycombe, UK). Both Cy3 $(532 \mathrm{~nm})$ and Cy5 $(635 \mathrm{~nm})$ channels were scanned at a photomultiplier setting of $65 \%$. Laser power was adjusted until there were no saturated spots. The images obtained (resolution 10 micron; 16 bit tiff image) were processed with ImaGene 5.0 software (Biodiscovery, Inc., Los Angeles, USA) to measure mean signal intensities for spots and local background. 


\section{Micramay Data Analyses}

Data were transferred to GeneSight 4.0 (Biodiscovery) for analysis. Flagged spots were excluded. For each spot, local background intensity was subtracted. The background corrected mean intensities were log transformed (base 2). Next, the expression difference for each spot was calculated by subtracting the log transformed mean intensity from the sample before the intervention from the log transformed mean intensity from the sample after the intervention. Expression differences were normalized using Lowess and the four replicates on each array of each gene were combined to a mean expression difference with exclusion of outliers ( $>2$ standard deviations).

To identify up- and down-regulated genes within a vegetable group $99.9 \%$ confidence intervals were calculated (method based on Kerr et $\mathrm{al}^{22}$ ). This very stringent interval was chosen to reduce the chance on false positive genes to $0.1 \%$. Cut-off levels were set at +0.25 and -0.25 respectively, because most of the genes were located within these boundaries.

The list of up- and down-regulated genes was further analyzed by means of the online software application Expression Analysis Systematic Explorer (EASE; http://david.niaid.nih.gov/david/ease.htm). ${ }^{23}$ EASE automates the process of biological theme determination and performs a statistical analysis of gene categories in the provided gene list to find those gene categories that are the most over-presented in the list of differentially expressed genes by a Fisher exact test.

\section{Additional Statistical Anatyses}

Statistical analysis of the questionnaires- and plasma-data was carried out using SPSS version 6.1.1 for Macintosh. The Shapiro-Wilks test was used to test normality of the datasets. Potential differences between the groups were assessed using the non-parametric Mann-Whitney U test or unpaired t-test. The non-parametric Wilcoxon Matched-Pairs Signed Ranks Test or the paired t-test was used to assess the differences within a vegetable group. All the tests were two-sided, and a p-value $<0.05$ was considered to indicate statistical significance.

\section{Results}

\section{Dietary Questionnaines and Plasma V alues}

The results of the dietary questionnaires and plasma values are presented in Table 1. Mean age of the patients was higher compared to controls $(p<0.01)$. No other significant differences between the groups before the intervention were observed. Mean vegetable intake was indeed approximately $150 \mathrm{~g} /$ day (in agreement with ${ }^{1}$ ), meaning that the subjects increased or decreased their intake during the intervention by a factor 2 . 


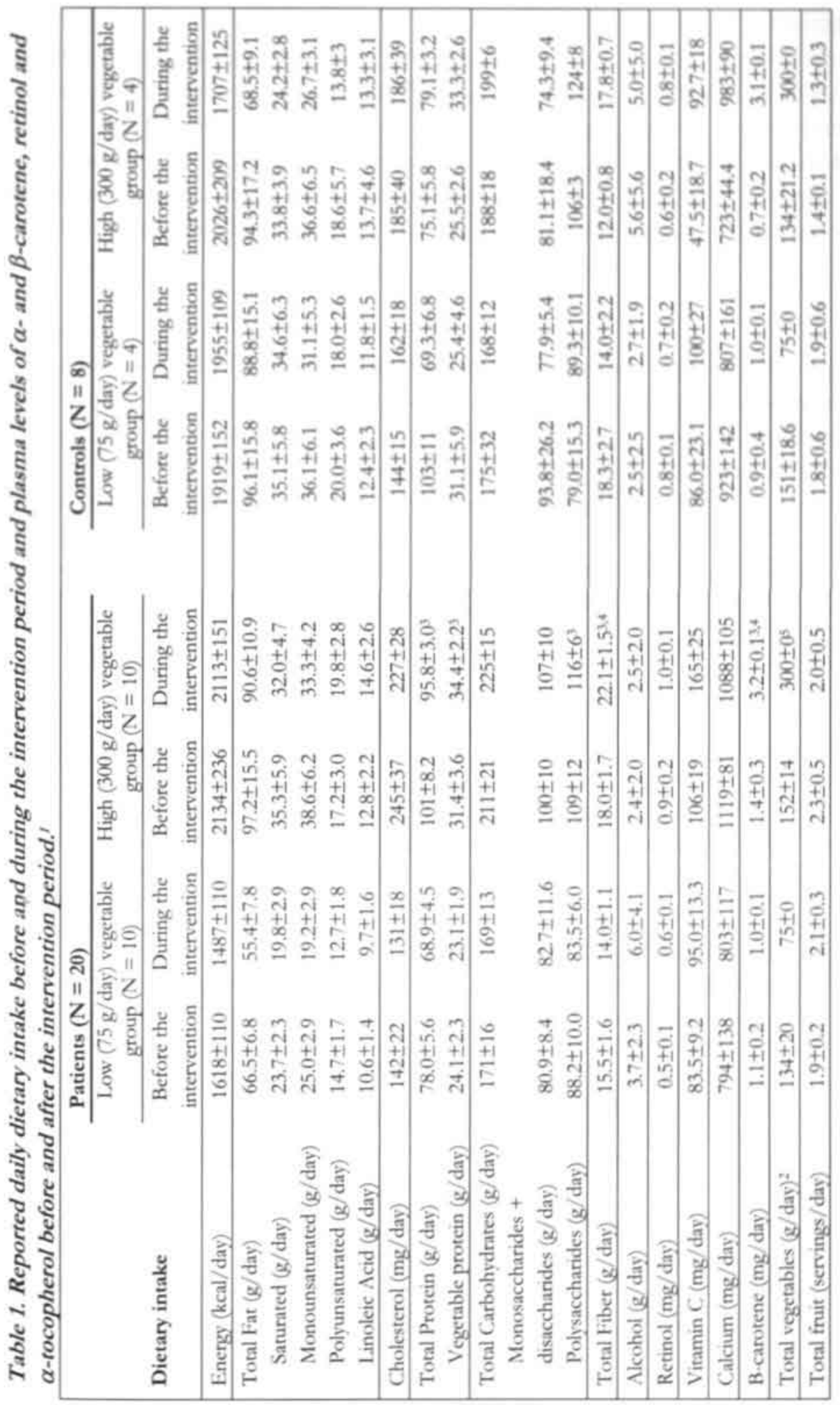


Table 1. (Continued) Reported daily dictary intake before and during the intervention period and plasma levels of $\alpha$-and $\beta$-carorene, retinol and $\alpha$-tocopherol before and after the intervention period.t

\begin{tabular}{|c|c|c|c|c|c|c|c|c|}
\hline \multirow[b]{3}{*}{ Plasma levels } & \multicolumn{4}{|c|}{ Patients $(\mathrm{N}=20)$} & \multicolumn{4}{|c|}{ Controls $(\mathrm{N}=8)$} \\
\hline & \multicolumn{2}{|c|}{$\begin{array}{c}\text { Low }(75 \mathrm{~g} / \text { day }) \text { vegetable } \\
\text { group }(\mathrm{N}=10)\end{array}$} & \multicolumn{2}{|c|}{$\begin{array}{c}\text { High ( } 300 \mathrm{~g} / \text { day) vegetable } \\
\text { group }\left(\mathrm{N}=10^{\circ}\right)\end{array}$} & \multicolumn{2}{|c|}{$\begin{array}{c}\text { Low }(75 \mathrm{~g} / \mathrm{day}) \text { vegetable } \\
\text { group }(\mathrm{N}=4)\end{array}$} & \multicolumn{2}{|c|}{$\begin{array}{c}\text { High }(300 \mathrm{~g} / \mathrm{day}) \text { vegetable } \\
\text { group }(\mathrm{N}=4)\end{array}$} \\
\hline & $\begin{array}{l}\text { Before the } \\
\text { intervention }\end{array}$ & $\begin{array}{l}\text { After the } \\
\text { intervention }\end{array}$ & $\begin{array}{l}\text { Before the } \\
\text { intervention }\end{array}$ & $\begin{array}{l}\text { After the } \\
\text { intervention }\end{array}$ & $\begin{array}{l}\text { Before the } \\
\text { intervention }\end{array}$ & $\begin{array}{l}\text { After the } \\
\text { intervention }\end{array}$ & $\begin{array}{l}\text { Before the } \\
\text { intervention }\end{array}$ & $\begin{array}{l}\text { After the } \\
\text { intervention }\end{array}$ \\
\hline$\alpha-$ - arotene $(\mu \mathrm{g} / \mathrm{ml})$ & $0.04 \pm 0.02$ & $0.05 \pm 0.01$ & $0.04 \pm 0.01$ & $0.12 \pm 0.02$ & $0.07 \pm 0.05$ & $0.07 \pm 0.04$ & $0.07 \pm 0.02$ & $0.13 \pm 0.04$ \\
\hline$\beta$-Carotene $(\mu \mathrm{g} / \mathrm{ml})$ & $0.23 \pm 0,04$ & $0.20 \pm 0.03$ & $0.22 \pm 0.04$ & $0.36 \pm 0.06^{6 . ?}$ & $0.29 \pm 0.15$ & $0.27 \pm 0.12$ & $0.25 \pm 0.11$ & $0.51 \pm 0.20$ \\
\hline Rerinol $(\mu \mathrm{g} / \mathrm{ml})$ & $0.96 \pm 0.11$ & $0.96 \pm 0.08$ & $0.82 \pm 0.06$ & $0.89 \pm 0.07$ & $0.83 \pm 0.02$ & $0.88 \pm 0.05$ & $0.94 \pm 0.10$ & $0.95 \pm 0.12$ \\
\hline$\alpha$ Tocopherol $(\mu \mathrm{g} / \mathrm{mI})$ & $15.3 \pm 1.6$ & $15.4 \pm 1.7$ & $13.9 \pm 1.6$ & $13.2 \pm 1.4$ & $10.70 \pm 1.43$ & $9.88 \pm 1.23$ & $1296 \pm 1.10$ & $1262 \pm 1.56$ \\
\hline
\end{tabular}

i Pluk-minus values are means \pm SEM.

IStandard vegrtable amount intake daring the intervention period.

ip<0.001; significantly bigher than mean intake dunng the intervention in the $75 \mathrm{~g}$ vegetable group.

" $P<0.001$; significandly hiphet than mean intake befote the intervention in the $300 \mathrm{~g}$ vegetable group.

$\mathrm{P}<0,0001$, significantly hagher than mean intake during the intervention in the $75 \mathrm{~g}$ vegetable group and before the intervention in the $300 \mathrm{~g}$ vegetable group

e $\mathrm{P}<0.05$; significantly higher than mean plasma level in the $75 \mathrm{~g}$ vegetable group after the intervention.

Pe 0.01, uprificantly higher than mean plasma intake in the $300 \mathrm{~g}$ vegetable group before the mintervention. 
The intake of total fiber, $\beta$-carotene $(\mathrm{P}<0.001)$ and total vegetables $(\mathrm{P}<0.0001)$ was significantly higher during the intervention as compared to the intake before the intervention in the high vegetable group. No significant changes in intake of other dietary compounds or life style habits were recorded. After the intervention, $\alpha$ - and $\beta$-carotene levels in plasma were significantly elevated in the high vegetable group compared to the levels before the intervention in the high vegetable group $(\mathrm{P}<0.01)$. For controls, similar findings were observed; however, because of the smaller number of subjects, differences were not significant.

\section{Gene Expression}

Table 2 shows a list of up- and down-regulated genes for each group. The expression differences of all genes are plotted against the mean expression in the pre-intervention samples in Figures 1A-1D. These scatter plots reveal that most genes are located within a range of $-0.25-+0.25$, only a few are located outside these boundaries and were identified as significantly up- or down-regulated by the confidence analysis.

Table 2. Genes significantly up- and down-regulated in the vegetable groups (confidence interval 99.9\%; up-and down-regulation level: $>0.25$, and $<-0.25$, respectively).

\begin{tabular}{|c|c|c|c|c|c|}
\hline \multirow[t]{2}{*}{$A C \# 1$} & \multirow{2}{*}{ 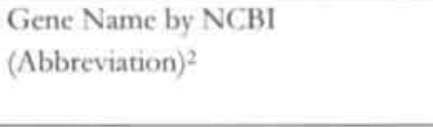 } & \multicolumn{2}{|c|}{ Controls } & \multicolumn{2}{|c|}{ Patients } \\
\hline & & $\begin{array}{l}\text { Expression } \\
\text { level }^{3}\end{array}$ & Difference ${ }^{4}$ & $\begin{array}{l}\text { Expression } \\
\text { |evel }\end{array}$ & Difference ${ }^{4}$ \\
\hline \multicolumn{6}{|c|}{ Up-regulation low (75 g/day) vegetable group } \\
\hline NM_000477 & Albumin (ALB) & & & $2.98 \pm 0.36$ & $0.44 \pm 0.56$ \\
\hline X03350 & $\begin{array}{l}\text { Alcohol dehydrogenase beta-1. } \\
\text { subunit (ADH1-2) }\end{array}$ & & & $8.66 \pm 0.22$ & $0.49 \pm 0.15$ \\
\hline M24317 & $\begin{array}{l}\text { Alcohol dehydrogenase beta-1- } \\
\text { subunit ( } \mathrm{ADH} 2 \text { ) }\end{array}$ & & & $9.97 \pm 0.19$ & $0.45 \pm 0.13$ \\
\hline XM_063498 & Alpha-fetoprotein (AFP) & & & $3.25 \pm 0.34$ & $0.38 \pm 0.36$ \\
\hline XM_005207 & $\begin{array}{l}\text { Carbonic anhydrase III, muscle } \\
\text { specific (CA3) }\end{array}$ & & & $2.85 \pm 0.39$ & $0.72 \pm 0.46$ \\
\hline XM_001734 & $\begin{array}{l}\text { Prostaglandin-endoperoxide } \\
\text { synthase } 2 \text { (PTGS2) }\end{array}$ & & & $6.80 \pm 0.13$ & $0.54 \pm 0.12$ \\
\hline 1.01131 & Decorin gene, exon 8 (DCN) & & & $8.68 \pm 0.21$ & $0.54 \pm 0.10$ \\
\hline M98478 & Human transglutaminase (TGM2) & & & $7.80 \pm 0.13$ & $0.58 \pm 0.13$ \\
\hline XM_009010 & Complement component $\mathrm{C} 3(\mathrm{C} 3)$ & $6.20 \pm 0.44$ & $0.68 \pm 0.19$ & $6.84 \pm 0.37$ & $0.47 \pm 0.40$ \\
\hline XM_012575 & Metallothionein IG (MTIG) & $9.88 \pm 0.37$ & $0.93 \pm 0.45$ & $9.69 \pm 0.26$ & $0.93 \pm 0.28$ \\
\hline$\times 97260$ & $\begin{array}{l}\text { Metallothionein isoform } 2 \\
\text { (MT2A) }\end{array}$ & $10.21 \pm 0.31$ & $0.86 \pm 0.38$ & $10.14 \pm 0.26$ & $0.84 \pm 0.28$ \\
\hline XM_003872 & $\begin{array}{l}\text { Methylacyl-CoA racemase alpha } \\
\text { (AMACR) }\end{array}$ & $9.74 \pm 0.15$ & $0.99 \pm 0.18$ & $9.90 \pm 0.16$ & $0.61 \pm 0.24$ \\
\hline NM_002738 & Protein kinase $C$ betal (PKCB1) & $4.31 \pm 0.28$ & $0.81 \pm 0.16$ & $4.93 \pm 0.16$ & $0.43 \pm 0.16$ \\
\hline
\end{tabular}




\section{Table 2. (Continued)}

\begin{tabular}{|c|c|c|c|c|c|}
\hline \multirow[t]{2}{*}{$\mathrm{AC \# 1}$} & \multirow{2}{*}{$\begin{array}{l}\text { Gene Name by NCBI } \\
\text { (Abbreviation) }^{2}\end{array}$} & \multicolumn{2}{|c|}{ Controls } & \multicolumn{2}{|c|}{ Patients } \\
\hline & & $\begin{array}{c}\text { Expression } \\
\text { level }\end{array}$ & Differencet & $\begin{array}{l}\text { Expression } \\
\text { leved }^{3}\end{array}$ & Difference ${ }^{4}$ \\
\hline \multicolumn{6}{|c|}{ Up-regulation low $(75 \mathrm{~g} /$ day) vegetable group } \\
\hline K01396 & $\begin{array}{l}\text { Serine (or cysteine) proteinase } \\
\text { inhibitor, clade } A \text {, member } 1 \\
\text { (SERPINA1) }\end{array}$ & $8.92 \pm 0.36$ & $0.68 \pm 0.23$ & & \\
\hline M13699 & Ceruloplasmin (ferroxidase) (CP) & $2.20 \pm 0.52$ & $0.88 \pm 0.21$ & & \\
\hline M61857 & $\begin{array}{l}\text { Cytochrome P450, family } 2 \text {, } \\
\text { subfamily C, polypeptide } 9 \\
\text { (CYP2C9) }\end{array}$ & $2.34 \pm 0.26$ & $1.80 \pm 0.70$ & & \\
\hline AF016641 & $\begin{array}{l}\text { Homo sapiens MHC class I } \\
\text { antigen HI_A-B }(\text { HL.A-B*1537) }\end{array}$ & $13.69 \pm 0.18$ & $0.65 \pm 0.26$ & & \\
\hline NML005957 & $\begin{array}{l}\text { 5,10-methylenetetrahydrofolate } \\
\text { reductase (MTHFR) }\end{array}$ & $5.22 \pm 0.33$ & $0.58 \pm 0.10$ & & \\
\hline XM 045678 & $\begin{array}{l}\text { Mitochondrial ribosomal protein } \\
\text { S11 (MRPS11) }\end{array}$ & $9.66 \pm 0.25$ & $0.63 \pm 0.24$ & & \\
\hline$\$ 69738$ & $\begin{array}{l}\text { Monocyte chemotactic protein-1 } \\
\text { (Aezo, }\end{array}$ & $5.57 \pm 0.09$ & $0.97 \pm 0.48$ & & \\
\hline $\mathrm{X} 14474$ & $\begin{array}{l}\text { Microtubule-associated tau } \\
\text { protein (MAPT) }\end{array}$ & $2.36 \pm 0.35$ & $1.39 \pm 0.61$ & & \\
\hline$\times 71354$ & $\begin{array}{l}\text { Vesicular monoamine transporter } \\
\text { (VMAT) }\end{array}$ & $5.34 \pm 0.53$ & $0.34 \pm 0.05$ & & \\
\hline \multicolumn{6}{|c|}{ Up-regulation high ( $300 \mathrm{~g} /$ day) vegetable group } \\
\hline $\mathrm{x} 03350$ & $\begin{array}{l}\text { Alcohol dehydrogenase beta-1. } \\
\text { subunit (ADH1-2) }\end{array}$ & & & $8.87 \pm 0.20$ & $0.45 \pm 0.10$ \\
\hline U33199 & Human mdm2-A (MDM2) & & & $2.73 \pm 0.27$ & $0.41 \pm 0.18$ \\
\hline XM 009010 & Complement component $\mathrm{C}_{3}(\mathrm{C} 3)$ & & & $6.19 \pm 0.42$ & $0.51 \pm 0.25$ \\
\hline D78341 & Cyclin G1 (CCNG1) & $8.27 \pm 0.21$ & $0.31 \pm 0.14$ & $8.68 \pm 0.23$ & $0.42 \pm 0.09$ \\
\hline 1.01131 & Decorin gene, exon 8 (DCN) & $8.36 \pm 0.51$ & $0.56 \pm 0.20$ & $8.29 \pm 0.29$ & $0.59 \pm 0.13$ \\
\hline XM_012575 & Metallothionein IG (MTIG) & $9.69 \pm 0.43$ & $0.74 \pm 0.41$ & $9.83 \pm 0.16$ & $0.53 \pm 0.27$ \\
\hline$\times 97260$ & $\begin{array}{l}\text { Metallothionein isoform } 2 \\
\text { (MTA2) }\end{array}$ & $10.08 \pm 0.4$ & $0.71 \pm 0.37$ & $10.23 \pm 0.13$ & $0.47 \pm 0.27$ \\
\hline $\mathrm{AB} 032261$ & $\begin{array}{l}\text { Stearyl-CoA desaturase, Liver } \\
\text { (SCD) }\end{array}$ & $3.73 \pm 0.46$ & $0.59 \pm 0.21$ & & \\
\hline $\mathrm{AB} 005038$ & $\begin{array}{l}\text { Cytochrome P450, family } 27 \text {. } \\
\text { subfamily B, polypeptide } 1 \\
\text { (CYP27B1) }\end{array}$ & $6.62 \pm 0.60$ & $0.51 \pm 0.10$ & & \\
\hline 1.19871 & $\begin{array}{l}\text { Activating transcription factor } 3 \\
\text { (ATF3) }\end{array}$ & $6.60 \pm 0.57$ & $0.64 \pm 0.32$ & & \\
\hline M29882 & Apolipoprotein AII (APOA2) & $4.92 \pm 0.54$ & $0.61 \pm 0.24$ & & \\
\hline XM_001859 & C-reactive protein (CRP) & $3.31 \pm 0.57$ & $0.44 \pm 0.23$ & & \\
\hline XM 001578 & E-selectin (SEI.E) & $2.89 \pm 0.52$ & $0.34 \pm 0.14$ & & \\
\hline
\end{tabular}


Table 2. (Continued)

\begin{tabular}{|c|c|c|c|c|c|}
\hline \multirow[t]{2}{*}{$A C \# 1$} & \multirow{2}{*}{$\begin{array}{l}\text { Gene Name by NCBI } \\
\text { (Abbreviation) }\end{array}$} & \multicolumn{2}{|c|}{ Controls } & \multicolumn{2}{|c|}{ Paticnts } \\
\hline & & $\begin{array}{l}\text { Expression } \\
\text { kevel }\end{array}$ & Differencet & $\begin{array}{l}\text { Expression } \\
\text { level }\end{array}$ & Differencet \\
\hline \multicolumn{6}{|c|}{ Down-regulation low $(75 \mathrm{~g} /$ day) vegetable group } \\
\hline NM_002417 & Ki67 antigen (MKI67) & & & $4.84 \pm 0.16$ & $-0.74 \pm 0.14$ \\
\hline M10617 & $\begin{array}{l}\text { Liver fatry acid binding protein } \\
\text { (L-FABP) }\end{array}$ & & & $12.90 \pm 0.17$ & $-0.53 \pm 0.14$ \\
\hline U33199 & Human mdm2-A (MDM2) & & & $3.46 \pm 0.31$ & $.0 .31 \pm 0.17$ \\
\hline NM_020299 & $\begin{array}{l}\text { Addo-keto reductase family 1, } \\
\text { member B10 (AKR1B10) }\end{array}$ & $4.78 \pm 0.57$ & $-1.05 \pm 0.36$ & $4.75 \pm 0,22$ & $-0.57 \pm 0.34$ \\
\hline AF016582 & Checkpoint kinase-1 (CHK-1) & $6.06 \pm 0.31$ & $-0.71 \pm 0.14$ & $6.47 \pm 0.19$ & $-0.70 \pm 0.24$ \\
\hline $\mathrm{X} 51688$ & Cyclin A2 (CCNA2) & $7.01 \pm 0.33$ & $-0.86 \pm 0.35$ & $7.20 \pm 0.26$ & $-0.52 \pm 0.17$ \\
\hline j05016 & $\begin{array}{l}\text { Disulfide isomerase telated } \\
\text { protein (ERp72) }\end{array}$ & $10.19 \pm 0.25$ & $-0.68 \pm 0.18$ & $10.22 \pm 0.18$ & $-0.49 \pm 0.09$ \\
\hline A) 271729 & $\begin{array}{l}\text { Glucose-regulated protein } \\
\text { (HSPA5 gene) }\end{array}$ & $11.31 \pm 0.43$ & $0.70 \pm 0.17$ & $11.21 \pm 0.19$ & $-0.56 \pm 0.18$ \\
\hline NM_000558 & Hemoglobin alpha I (HBAI) & $7.58 \pm 0.38$ & $-0.67 \pm 0.11$ & $7.53 \pm 0.21$ & $-0.53 \pm 0.12$ \\
\hline ABC05038 & $\begin{array}{l}\text { Cytochrome P450, family } 27 \text {, } \\
\text { subfamily B, polypeptide } 1 \\
\text { (CYP27B1) }\end{array}$ & $7.34 \pm 0.40$ & $-1.06 \pm 0.06$ & & \\
\hline XM_001393 & Peroxiredoxin 1 (PRDX1) & $7.55 \pm 0.28$ & $-1.14 \pm 0.60$ & & \\
\hline XM_004453 & $\begin{array}{l}\text { Homo sapiens } \mathrm{H} 2 \mathrm{~A} \text { histone } \\
\text { family. member } \mathrm{I}(\mathrm{H} 2 \mathrm{AFI})\end{array}$ & $12.02 \pm 0.24$ & $-0.70 \pm 0.18$ & & \\
\hline XM_004419 & $\begin{array}{l}\text { Homo sapiens } \mathrm{H} 3 \text { histone family. } \\
\text { member } \mathrm{B}(\mathrm{H} 3 \mathrm{FB})\end{array}$ & $13.31 \pm 0.27$ & $-0.64 \pm 0.11$ & & \\
\hline AJ001417 & $\begin{array}{l}\text { Solute carrier family } 22 \\
\text { (extraneuronal monoamine } \\
\text { transporter). member } 3 \\
\text { (SLC22A3) }\end{array}$ & $7.47 \pm 0.11$ & $-1.47 \pm 0.11$ & & \\
\hline $\mathrm{X} 0230 \overline{8}$ & Thymidylate synthase (TYMS) & $8.15 \pm 0.19$ & $0.65 \pm 0.07$ & & \\
\hline \multicolumn{6}{|c|}{ Down-regulation high ( $300 \mathrm{~g} /$ day) vegetable group } \\
\hline M16650 & Ornithine decarboxylase (ODC1) & $9.11 \pm 0.35$ & $-1.41 \pm 0.44$ & $8.65 \pm 0.27$ & $-0.78 \pm 0.17$ \\
\hline K00650 & Fos proto-oncogene (C-FOS) & $7.46 \pm 0.54$ & $-2.07 \pm 0.54$ & $6.80 \pm 0.48$ & $-0.87 \pm 0.19$ \\
\hline M10617 & $\begin{array}{l}\text { Liver fatty acid binding protein } \\
\text { (L-FABP) }\end{array}$ & $12.82 \pm 0.10$ & $-0.75 \pm 0.37$ & & \\
\hline AF 057039 & $\begin{array}{l}\text { Homo sapiens putative renal } \\
\text { organic anion transporter } 1 \\
\text { (hROAT1) }\end{array}$ & $5.42 \pm 0.46$ & $-0.67 \pm 0.22$ & & \\
\hline 104718 & $\begin{array}{l}\text { Proliferating cell nuclear antigen } \\
\text { gene (PCNA) }\end{array}$ & $9.10 \pm 0.16$ & $-1.15 \pm 0.22$ & & \\
\hline M61854 & $\begin{array}{l}\text { Cytochrome P450, family } 2 \text {, } \\
\text { subfamily C, polypeptide } 19 \\
\text { (CYP2C19) }\end{array}$ & $3.85 \pm 0.28$ & $-1.52 \pm 0.50$ & & \\
\hline
\end{tabular}


Table 2. (Continued)

\begin{tabular}{|c|c|c|c|c|c|}
\hline \multirow[t]{2}{*}{$A C \# 1$} & \multirow{2}{*}{$\begin{array}{l}\text { Gene Name by NCBI } \\
\text { (Abbreviation) })^{2}\end{array}$} & \multicolumn{2}{|c|}{ Controls } & \multicolumn{2}{|c|}{ Patients } \\
\hline & & $\begin{array}{c}\text { Expression } \\
\text { level }^{5}\end{array}$ & Differencet $^{4}$ & $\begin{array}{c}\text { Expression } \\
\text { level }^{3}\end{array}$ & Difference $^{4}$ \\
\hline \multicolumn{6}{|c|}{ Down-regulation high $(300 \mathrm{~g} /$ day $)$ vegetable group } \\
\hline Xo8006 & $\begin{array}{l}\text { Cytochrome P450, family } 2 \text {, } \\
\text { subfamily D, polypeptide } 6 \\
\text { (CYP2D6) }\end{array}$ & $4.46 \pm 0.32$ & $-2.18 \pm 0.84$ & & \\
\hline M18907 & $\begin{array}{l}\text { Cytochrome P450, family } 3 \text {, } \\
\text { subfamily A, polypeptide } 4 \\
\text { (CYP3A4) }\end{array}$ & $4.76 \pm 0.63$ & $-1.80 \pm 0.76$ & & \\
\hline
\end{tabular}

'ACH: GenBank accession numbers of the cDNA fragments present on the microarrays.

NCBI (National Center for Biotechnology Informarion): htrps//www.ncbinlen.nih.gov/.

Wixpression level is mean Jog intensiry from the samples before the intervention. Plus-minus values are means \pm SEM.

"Up- and down regulation level: $\geq 0.25$ and $\leq-0.25$, respectively, Difference is the mean "log difference (the Plog transformed mean intensity from the samples before the intervention subtracted from the Jlog transformed mean intensity from the samples after the intervention) within a vegerable group.

The complete differential gene list was analyzed using E.ASE. Taken all differentially expressed genes into account (52), regardless of subject group and diet, 13 gene categories were significantly over-presented: four out of the five significantly overpresented biological processes are related to cell cycle control and seven of the eight molecular functions comprise oxidoreductase activities. Splitting the genes by means of subject group, the cell cycle control effect remains significant. The significant effect of the oxidoreductase activity category remains only in the $300 \mathrm{~g}$ vegetable control group. According to literature review of all genes, a lot of the genes which are related to (colon)carcinogenesis are present in the significant gene categories. In addition, genes which were not present in the significant gene categories, but are thought to be relevant to (colon) carcinogenesis according to the literature review are also taken into account.

Genes with different functions were modulated, only the 20 genes which are related to (colon)carcinogenesis are explained in more detail in Table 3. Seven of these genes were similarly modulated in both patients and controls. Methyl-CoA racemase alpha (AMACR) and protein kinase $\mathrm{C}$ beta 1 (PKCB1) were up-regulated in the low vegetable group; cyclin A2 (CCNA2) and checkpoint kinase-1 (CHK1) were down-regulated. In the high vegetable group, cyclin G1 (CCNG1) was up-regulated, and fos proto-oncogene (CFOS) and ornithine decarboxylase 1 (ODC1) were down-regulated. Other genes which were modulated in either patients or controls include alpha-fetoprotein (AFP), prostaglandin-endoperoxide synthase 2 (PTGS2) or cyclooxygenase-2 (COX-2), cytochrome P450 2C9 (CYP2C9), Ki67 antigen, 5,10-methylenetetrahydrofolate reductase (MTHFR), activating transcription factor 3 (ATF3), Human mdm2-A (MDM2), cytochrome P450, family 27, subfamily B, polypeptide 1 (CYP27B1), peroxiredoxin 1 
(PRDX1), proliferating cell nuclear antigen gene (PCNA), cytochrome P450 2 C19 (CYP2C19), -2D6 (CYP2D6) and -3A4 (CYP3A4).

\section{A: Patients $75 \mathrm{~g} /$ day}

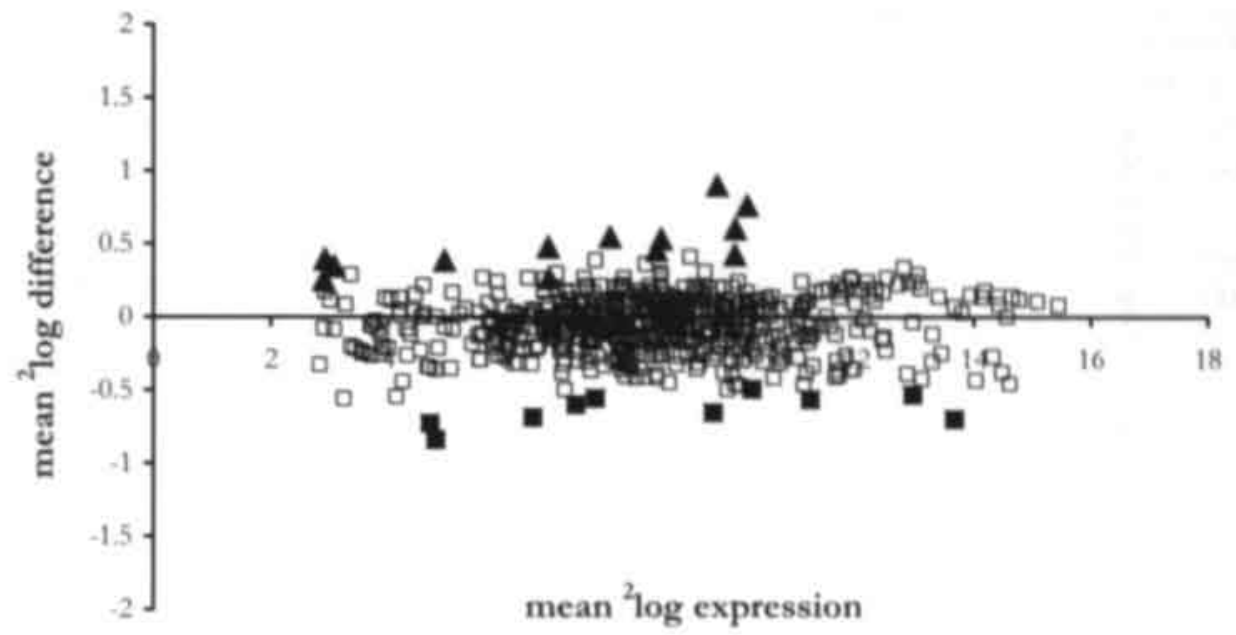

\section{B: Patients $300 \mathrm{~g} /$ day}

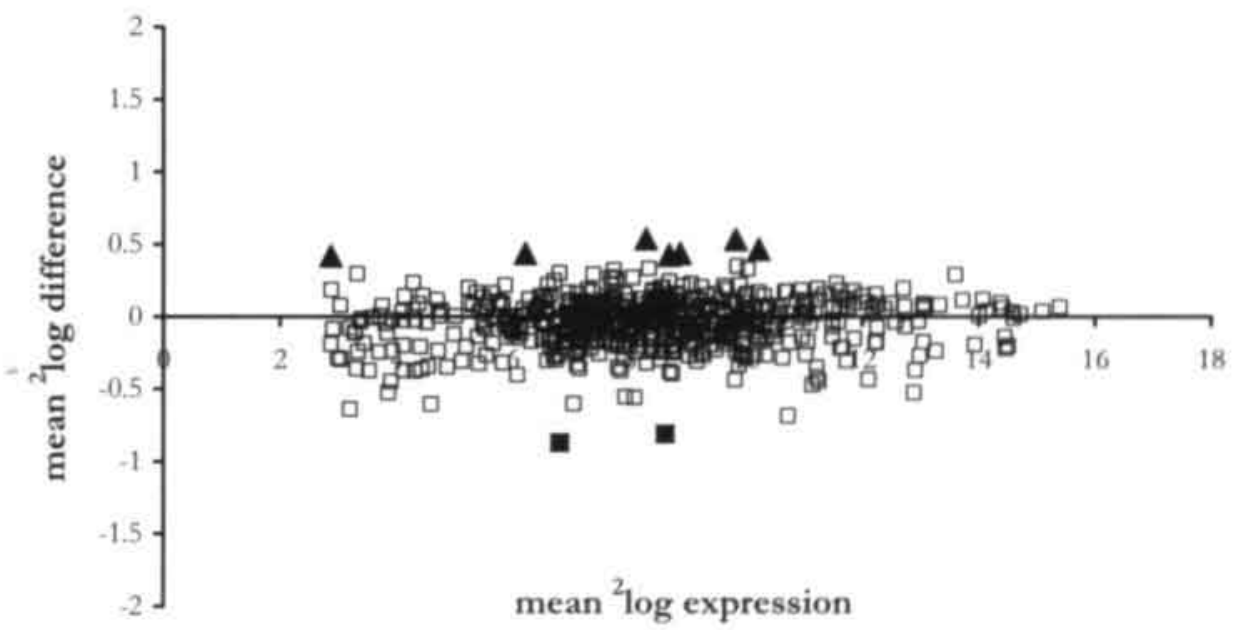

Figure 1. Scatter plot views of gene expression: Mean Jog expression intensities before the intervention versus mean Jog difference. Black squares and trangles indicate statistically significant genes whit a down-regulation level of -0.25 and upregulanon level of 0.25 , respectuvely as determined by means of $99.9 \%$ confidence intervals. Transparent squares indicate genes that are not significantly up- or down-regulated. ( $\Lambda$ ) Scatter plot view of genes in the low vegetable panent group: 13 genes are up-regulated; 10 genes are down-regulated. (B) Scarter plot view of genes in the high vegetable patient group: 7 genes are up-regulated; 2 genes are down-regulated. (C) Scarter plot view of genes in the low vegetable control group: 15 genes are up-regulated: 12 genes are down-regulated. (D) Scatter plot view of genes in the high vegetable control groups 10 genes are up-regulated; 8 genes are down-regulated. 
C: Controls $75 \mathrm{~g} /$ day

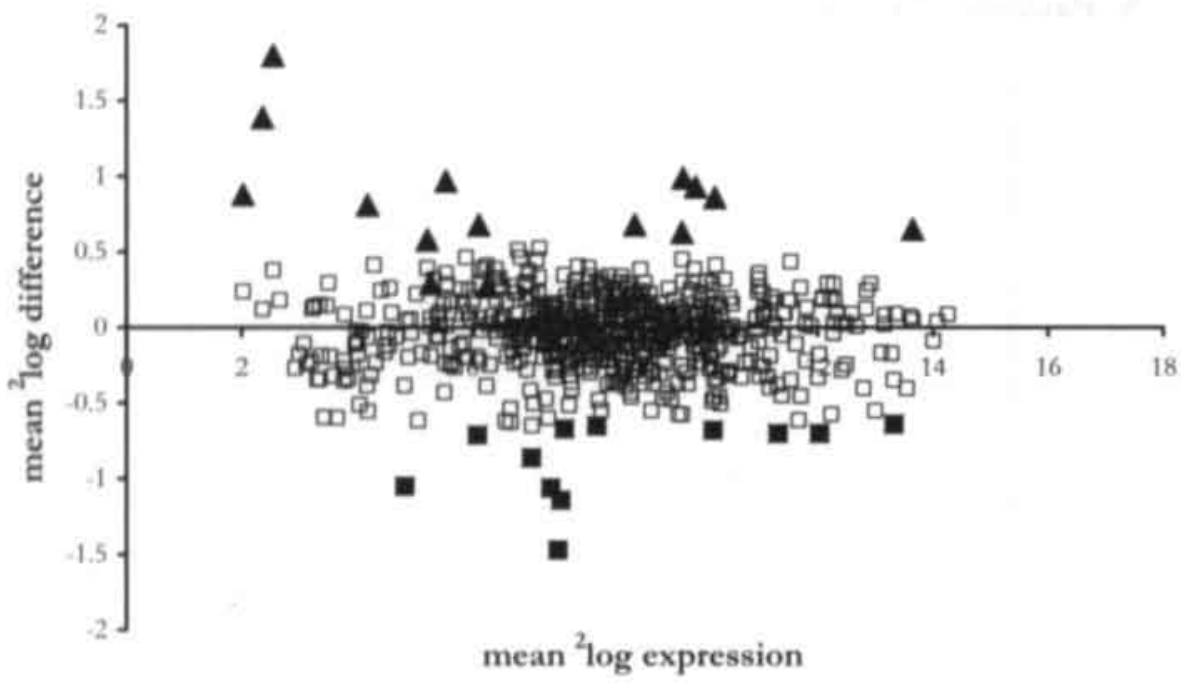

D: Controls $300 \mathrm{~g} /$ day

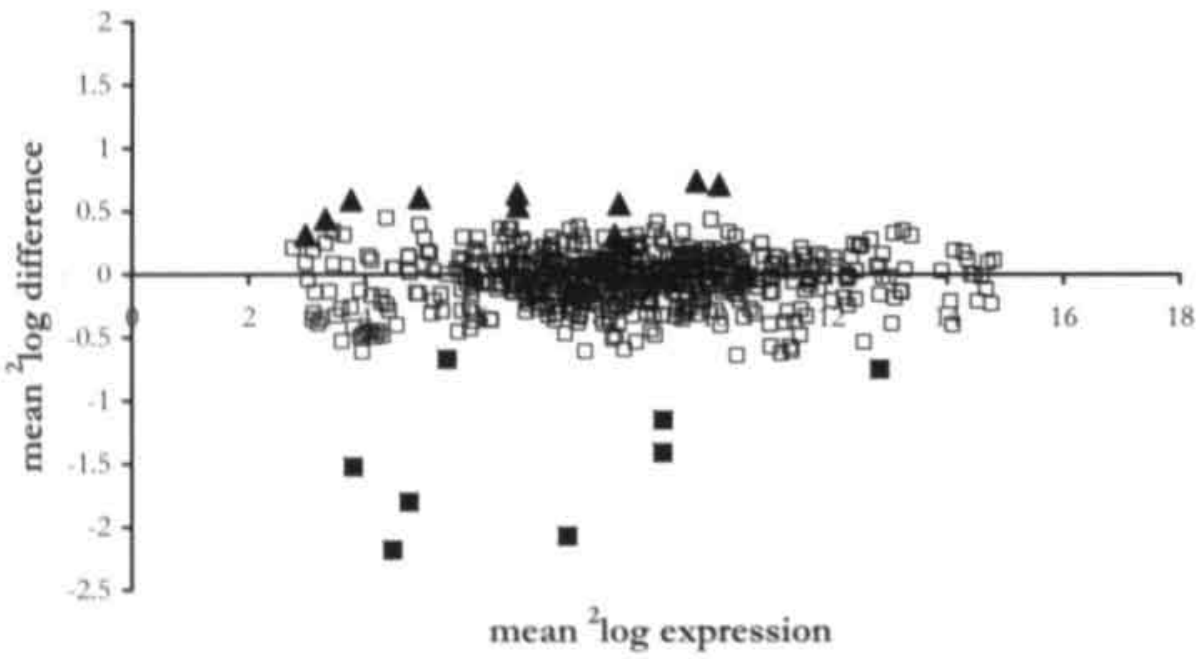

Figure 1. (Continued) 


\section{Table 3. Function of differentially expressed genes known to be involved in CRC.}

\begin{tabular}{|c|c|}
\hline Abbre & Function in relation to carcinogenesis ${ }^{2}$ \\
\hline AFP & $\begin{array}{l}\text { Tumor associated fetal protein that primarily promotes growth in in nitw and in wiv } \\
\text { models. AFP can serve as a dual regulator of cell proliferation (up- and down-rogulation } \\
\text { of growth) }\end{array}$ \\
\hline PTGS2 & $\begin{array}{l}\text { Converts arachidonic acid into bioactive prostanoids that are believed to play a role in } \\
\text { carcinogenesis by promoting cell proliferation and neoangiogenesis, inhibiting apoptosis }\end{array}$ \\
\hline AMACR & $\begin{array}{l}\text { Plays an important role in bile acid biosynthesis and } \beta \text {-oxidation of branched farty acids. } \\
\text { Overexpression of AMACR occurs in tumors that have been linked to high fat diet such } \\
\text { as prostate and colon cancer }\end{array}$ \\
\hline PKCB1 & $\begin{array}{l}\text { Isoform of serine/threonine protein kinases, which is involved in signal transduction } \\
\text { pathways and regulates growth and differentiation in eukaryotic cells. PKC isoforms are } \\
\text { often overexpressed in cancer }\end{array}$ \\
\hline CCNG1 & $\begin{array}{l}\text { Cyclins bind to cyclin dependent protein kinases (CDK), which control the cell cycle. } \\
\text { There is no proven CDK partner for cyclin G1. Conflicting roles have been assigned to } \\
\text { CCNGI in growth control }\end{array}$ \\
\hline CCNA2 & $\begin{array}{l}\text { CCNA2 activates two different CDKs and is associated with cellular proliferation. } \\
\text { CCNA2 is overexpressed in many types of cancers and is associated with poor prognosis. }\end{array}$ \\
\hline MDM2 & $\begin{array}{l}\text { Nuclear phosphoprotein which forms a complex with p } 53 \text {. It regulates p } 53 \text { by targeting its } \\
\text { destruction }\end{array}$ \\
\hline CHK-1 & $\begin{array}{l}\text { CHK-1 is an essential component of the DNA damage and -replication checkpoint. It } \\
\text { directly phophorylates Cde } 25 \mathrm{~A} \text { protein phosphatase during an unperturbed cell cycie that } \\
\text { is required for cells to delay cell cycle progression in response to double strand breaks }\end{array}$ \\
\hline K167 & $\begin{array}{l}\text { Marker for tumor proliferation rate. It provides estimates of the growth fraction of many } \\
\text { normal and malignant tissues, including the colon }\end{array}$ \\
\hline ODCl & $\begin{array}{l}\text { First enzyme in polyamine synthesis, Polyamine metabolism is an integral component of } \\
\text { carcinogenesis in epithelial tissues. There is a strong association between high levels of } \\
\text { polyamines and rapid proliferation. Increases in ODC are often associated with initiation } \\
\text { of normal cell growth and with sustained neoplastic cell growth }\end{array}$ \\
\hline C.FOS & $\begin{array}{l}\text { This proto-oneogene has been implicated in cell growth, differentiation and development. } \\
\text { It is classified as an early growth-regulated gene }\end{array}$ \\
\hline CYP2C.9 & $\begin{array}{l}\text { Phase- } 1 \text { biotransformation enzyme, capable of metabolizing a number of clinically drugs } \\
\text { and of metabolically activating polycyclic aromatic hydrocarbons and heterocyclic } \\
\text { aromatic amines }\end{array}$ \\
\hline MTHFR & $\begin{array}{l}\text { Regulates metabolism of folate, Low activity of this enzyme is associated with reduced } \\
\text { colon cancer risk. }\end{array}$ \\
\hline CYP27B1 & $\begin{array}{l}\text { This gene encodes for an enzyme which is responsible for the conversion of vitamin } D \text { to } \\
\text { the active hormonal metabolite } 1,25 \text {-dihydroxyvitamin } D_{3}\left(1,25-D_{3}\right) \text { which may act as } \\
\text { antimitotic and prodifferentiating agent }\end{array}$ \\
\hline ATF3 & $\begin{array}{l}\text { ATF } 3 \text { is an immediate carly response gene that is induced in cells exposed to a variety of } \\
\text { stress stimuli. It is a target gene of p } 53 \text {. The role of ATF } 3 \text { in carcinogenesis is not clear }\end{array}$ \\
\hline PRDX1 & $\begin{array}{l}\text { Antioxidant enzyme, which reduces hydrogen peroxide and alkyl hydroperoxides and } \\
\text { thereby functions as a tumor suppressor }\end{array}$ \\
\hline
\end{tabular}


Table 3. (Continued)

\begin{tabular}{|ll|}
\hline Abbreviation' & Function in relation to carcinogenesis ${ }^{2}$ \\
\hline PCNA & $\begin{array}{l}\text { Marker for tumor proliferation rate. It provides estimates of the growth fraction of many } \\
\text { normal and malignant tissues, including the colon }\end{array}$ \\
\hline CYP2C19 & Phase-1 biotransformation enzyme, metabolizes a number of prescribed drugs \\
\hline CYP2D6 & Phase-1 biotransformation enzyme, metabolizes a number of clinically important drugs \\
\hline CYP3A4 & $\begin{array}{l}\text { Phase-1 biotransformation enzyme, metabolizes clinically used drugs and carcinogens } \\
\text { such as aflatoxin B1, 6-aminochrysene, benzo[alpyrene. } \\
\\
\text { methylimidazo/4,5-f]quinoline (IQ) and 2-amino-3- }\end{array}$ \\
\hline
\end{tabular}

'NCBI (National Centet for Biotechnology Information): hrtp://www.ncbi.nim.nih.gov/.

${ }^{2}$ NCBI (National Center for Biotechnology Information): Locuslink and OMIM: hrrp://www.ncbinlm.nih.gov/.

\section{Discussion}

The current study describes the transcriptome changes induced in normal colorectal mucosa of female adenoma patients and healthy controls who received a decreased or increased amount of vegetables for a period of two weeks.

The results of the dietary questionnaires and plasma values support that the effects on gene expression found in this study truly are the result of changing the intake of vegetables, 17,24

The differences in expression level before and after the two-week intervention period were relatively small, but distinct as based on confidence analyses. Only the genes which are known to be related to (colon)carcinogenesis are discussed here. First, genes which were significantly modulated in both patients and controls are reviewed. Next, the most interesting genes which were significantly modulated in either patients or controls are discussed. Table 4 summarizes the effects on expression of these genes according to subject group and in relation to possible effects on carcinogenesis.

Seven genes were similarly modulated in both study groups, the patients and healthy controls, which substantiate the importance of dietary control on these genes. In the low vegetable group, the induction of $\mathrm{AMACR}$ and $\mathrm{PKCB} 1$ and reduction of CHK1 and CCNA2 can be explained by a decreased intake of inhibitors or stimulators, respectively. Overexpression of AMACR is apparent in tumors that have been linked to high-fat diet such as CRC. As AMACR is involved in lipid metabolism it is speculated that this could lead to alterations in oxidant balance of a cell leading to DNA damage, malignant transformation and other parameters of cell disturbance. ${ }^{25} \mathrm{PKC}$ isoforms are often overexpressed in disease states such as cancer. ${ }^{26}$ PKC activation has been implicated in tumor promotion of colonic epithelial cells by endogenous and dietary factors such as bile acids and free fatty acids. ${ }^{27}$ CHK1 is an essential component of the DNA damage and 
-replication checkpoint. It is required for cells to delay cell cycle progression in response to double strand breaks. ${ }^{28}$ CCNA2 can activate two different cyclin dependent protein kinases (CDKs) and is associated with cellular proliferation. It is overexpressed in many types of cancers with high proliferative activity and is associated with a poor prognosis. ${ }^{20}$. Thus the effects of reduced vegetable intake on AMACR, PKCB1 and CHK1 are expected to enhance cancer risk, whereas for CCNA2 to induce cancer risk suppression.

In the high vegetable group, CCNG1 was up-regulated, and C-FOS and ODC1 were down-regulated for both patients and controls. CCNG1 is a transcriptional target of p53 and its up-regulation coincides with activation of p53. Activated p53 suppresses growth by activating genes that trigger growth arrest or apoptosis. It is speculated that p53 activation is achieved by CCNG1 by stoichiometrically limiting the binding between $\mathrm{p} 53$ and MDM2, a down-regulator of p53, or by altering the phosphorylation status of MDM2 via phosphatase $\mathrm{A} 2{ }^{30}$ Noteworthy, also MDM2 expression is regulated by p53.00.32 For patients, down-regulation of MDM2 occurred in the low vegetable group, up-regulation of MDM2 and CCNG1 was observed in the high vegetable group. C-FOS protein interacts with the protein of another proto-oncogene, C-JUN to form a heterodimer. This complex

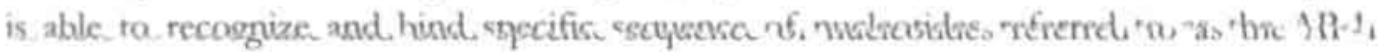
binding site located in the promoter region of genes which are expressed in transformed rapidly growing cells. ${ }^{33} \mathrm{C}-\mathrm{FOS}$ is overexpressed in the preneoplastic colonic lesions aberrant crypt foci. ${ }^{33}$ ODC1 is a transcriptional target of C-MYC and a modifier of adenomatous polyposis coli (APC)-dependent tumorigenesis. Most sporadic colon adenomas acquire mutations in the APC gene and show defects in APC-dependent signaling. Wild-type APC suppresses C-MYC, activates the C-MYC antagonist MAD1, and reduces the expression of ODC1 resulting in a decrease in polyamine synthesis and reduced tumorigenesis. $\beta$-Carotene inhibits ODC1 expression. ${ }^{34}$ Intake and plasma levels of $\beta$-carotene were significantly increased in the high vegetable group and could thus explain the ODC1 inhibition. For all these genes the modulatory effects of increased vegetable intake on their expression might result in a decreased cell growth and as a consequence in lower colon cancer risk.

Patients already have a genetic predisposition to develop adenomas as compared to controls and that could explain why we found 13 genes modulated differently in patients compared to controls. The most interesting ones are discussed here. Already mentioned is the effect of vegetables on MDM2 expression in patients. Furthermore, COX-2, upregulated in patients on low vegetable intake, is an established target for preventive interventions, as non-steroidal anti-inflammatory drugs (NSAIDs) have been shown to suppress colon carcinogenesis through the inhibition of COX-2. ${ }^{35}$ Inhibition of COX-2 expression by vegetable components has previously been reported. $.^{36,}, 3^{77}$ In a study of Plummer et al (1999), COX-2 induction by the colon tumor promoters tumor necrosis factor $\alpha$ or fecapentaene- 12 in human colon cells was diminished by the chemopreventive 
agent curcumin. ${ }^{36}$ Wenzel et al $(2000)$ found that that dietary flavone was able to diminish COX-2 mRNA in the human carcinoma cell line HT-29 after a $48 \mathrm{~h}$ incubation. ${ }^{37}$ In the present study, the observed up-regulation of COX-2 could be the result of a decreased intake of possible COX-2 inhibitors and agrees with the observed cancer risk suppression by dietary vegetables.

Table 4. Presentation of differentially expressed genes known to be involved in CRC, the direction of the effect, and the theoretical effect of this on cancer risk.

\begin{tabular}{|c|c|c|c|c|}
\hline \multirow{2}{*}{$\begin{array}{l}\text { Gene Name by } \\
\text { NCBI } \\
\text { (Abbreviation)! }\end{array}$} & \multicolumn{2}{|c|}{ Reduced vegetable intake } & \multicolumn{2}{|c|}{ Increased vegetable intake } \\
\hline & $\begin{array}{l}\text { Efffect on } \\
\text { expression }\end{array}$ & $\begin{array}{c}\text { Theoretical effect } \\
\text { on cancer risk }\end{array}$ & $\begin{array}{l}\text { Effect on } \\
\text { expression }\end{array}$ & $\begin{array}{c}\text { Theoretical effect on } \\
\text { cancer risk }\end{array}$ \\
\hline \multicolumn{5}{|c|}{ In patients and controls } \\
\hline AMACR & + & + & & \\
\hline PKCB1 & + & + & & \\
\hline CHKI & - & + & & \\
\hline CCNA2 & - & - & & \\
\hline CCNG1 & & & + & - \\
\hline C.FOS & & & - & - \\
\hline ODC1 & & & - & - \\
\hline \multicolumn{5}{|l|}{ In patients only } \\
\hline $\operatorname{cox}-2$ & + & + & & \\
\hline MDM/2 & & & + & $?$ \\
\hline \multicolumn{5}{|l|}{ In controls only } \\
\hline MDM2 & - & ? & & \\
\hline CYP2C9 & + & + & & \\
\hline CYP2C19 & & & - & ? \\
\hline CYP2D6 & & & - & $?$ \\
\hline CYP3A4 & & & - & - \\
\hline CYP27131 & - & + & + & - \\
\hline
\end{tabular}

'NCB1 (National Center for Biotechnology Information): http://www.ncbi.nim.nih.gov.

In controls, but not in patients, several phase-I biotransformation genes were modulated. In the low vegetable group CYP2C9 was up-regulated and in the high vegetable group CYP2C19,-2D6 and 3A4 were down-regulated. The CYP genes code for enzymes that function in a wide variety of metabolic pathways involving both endogenous and exogenous compounds. In endogenous processes, CYP enzymes are involved in the biosynthesis and catabolism of signaling molecules such as steroid hormones, retinoic acid, and vitamin D. ${ }^{38}$ CYP27B1 codes for the enzyme which converses vitamin D to the active hormonal metabolite 1,25-dihydroxyvitamin $\mathrm{D}_{3}$, which may act as an antimitotic and prodifferentiating agent in colon cancer cells. ${ }^{39}$ The expression of CYP27B1 was downregulated in the low and up-regulated in the high vegetable group. In addition to their role in endogenous processes, CYP enzymes catalyze the biotransformation of a wide variety of 
xenobiotic compounds which require metabolic activation to form ultimate carcinogens or toxicants. By blocking the formation of ultimate carcinogens, initiation of carcinogenesis can be prevented. 38 CYP2C9 38.40 and CYP3A4 are responsible for the metabolism of a number of drugs, and the (colon)carcinogens polycyclic aromatic hydrocarbons and heterocyclic aromatic amines. ${ }^{41}$ Also CYP2C19 and CYP2D6 metabolize a number of drugs, but have not been thoroughly studied in relation to cancer susceptibility, ${ }^{36}$ Once again, all these dietary effects on gene expression of CYP450 enzymes by altering vegetable intake agree with the presumed cancer-modulating properties of vegetables.

In conclusion, almost all the effects on the expression of genes by altering vegetable intake can be mechanistically linked to cellulat processes that explain prevention of colon-cancer risk by high vegetable intake or higher colon-cancer risk by low vegetable intake (see Table 4). The only exception to this is CCNA2, which maybe is involved in currently unknown but relevant processes. Furthermore, it seems that for patients, genes are modulated which are involved in the late stages of CRC like MDM2 and COX-2, whereas for controls genes are modulated involved in initiating events, i.e. the CYP450 genes. This human study is the first in which the vegetable intake was investigated to modulate the expression of genes involved in carcinogenesis in colorectal mucosa. An increased intake of vegetables resulted in down-regulation of genes promoting cell proliferation and bioactivation of procarcinogens, and in up-regulation of genes involved in cell growth arrest; in contrast, a decreased intake of vegetables results in downregulation of genes inhibiting cell growth and up-regulation of genes promoting cellular differentiation and bioactivation of procarcinogens. Some of the modulated genes are already targets in colon-chemoprevention trials, like COX-2. Possibly, the others are new targets for chemoprevention. Further research is needed to investigate what would be the relative impact of preventive modulation of these particular genetic pathways in reducing CRC risk. 


\section{References}

1. World Health Organization \& International Agency for Research on Cancer. World Cancer Report. Lyon. IARCPress, 2003.

2. Dashwood RH. Early detection and prevention of colorectal cancer (review). Oncol Rep 1999;6:27781.

3. World Cancer Research Fund. Food, Nutrition and the Prevention of Cancer: a Global Perspective. Chapter 4.10: Colon, rectum. Washington: BANTA Book group, 1997:216-251.

4. Potter JD, Slattery ML, Bostick RM, Gapstur SM. Colon cancer: a review of the epidemiology. Epidemiol Rev 1993;15:499-545.

5. Wattenberg LW, Inhibition of carcinogenesis by minor dietary constituents. Cancer Res 1992; $2: 2085$ s-2091s.

6. Manson MM, Gescher A, Hudson EA, Plummer SM, Squires MS, Prigent SA. Blocking and suppressing mechanisms of chemoprevention by dietary constituents. Toxicol Lett 2000,112113:499-505.

7. Reddy BS. The Fourth DeWitt S. Goodman lecture. Novel approaches to the prevention of colon cancer by nutritional manipulation and chemoprevention. Cancer Epidemiol Biomarkers Prev 2000;9:239-47.

8. Fearon ER, Vogelstein B. A genetic model for colorectal tumorigenesis. Cell 1990;61:759-67.

9. Arends JW. Molecular interactions in the Vogelstein model of colorectal carcinoma. J Pathol 2000;190:412-6.

10. Chung DC. The genetic basis of colorectal cancer: insights into critical pathways of tumorigenesis. Gastroenterology 2000;119;854-65.

11. Muller M, Kersten S. Opinion: Nutrigenomies; goals and strategies. Nat Rev Genet 2003;4:315-22.

12. Kall MA, Vang O, Clausen J. Effects of dietary broccoli on human in vivo drug metabolizing enzymes: evaluation of caffeine, oestrone and chlorzoxazone metabolism [see comments]. Carcinogenesis 1996;17:793-9.

13. Jampe JW, Chen C, Li S, Prunty J. Grate MT, Meehan DE, Barale KV, Dightman DA, Feng Z, Potter JD. Modulation of human glutathione S-transferases by botanically defined vegetable diets [In Process Citation]. Cancer Epidemiol Biomarkers Prev 2000;9:787-93.

14. Lampe JW, King IB, Li S, Grate MT, Barale KV, Chen C, Feng Z, Potter JD, Brassica vegetables increase and apiaceous vegetables decrease cytochrome P450 $1 \mathrm{A2}$ activity in humans: changes in caffeine metabolite ratios in response to controlled vegetable diets. Carcinogenesis 2000;,21:1157-62.

15. Nijhoff WA, Grubben MJ. Nagengast FM, Jansen JB, Verhagen H, van Poppel G, Peters WH. Effects of consumption of Brussels sprouts on intestinal and lymphocytic glutathione S-transferases in humans, Carcinogenesis 1995;16:2125-8.

16. Pool-Zobel BI, Bub $\mathrm{A}$, Liegibel UM, Treptow-van Lishaut S, Rechkemmer G. Mechanisms by which vegetable consumption reduces genetic damage in humans. Cancer Epidemiol Biomarkers Prev 1998;7:891-9.

17. Anonymous. Zo eet Nederland. Resultaten van de voedselconsumptiepeiling 1997-1998. Voedingscentrum Den Haag, 1998.

18. Gartner L.P, Hatt JL. Chapter 17: Digestive System: Alimentary Canal. Color textbook of histology, second edition. Philadelphia: W.B. Saunders Company, 2001:379-409. 
19. Hess D, Kellet HE, Oberin B, Bonfanti R, Schuep W. Simultaneous determination of retinol, tocopherols, carotenes and bycopene in plasma by means of high-performance liquid chromatography on reversed phase. Int J Vitam Nutr Res 1991;61:232-8.

20. NEVO Table: Dutch Food Composition Table. The Hague: Bureau for Nutritional Education, 1984.

21. Hasseman J, Chen E, Yang 1, Quackenbush J. Aminoallyl labeling of RNA for microarrays. htrp://www.tigr.org/tdb/micoarray/protocolsT1GR,shtml (Accessed June 21, 2001), 2001.

22. Kerr MK, Martin M, Churchill GA. Analysis of variance for gene expression microarray data. Journal of Computational Biology 2000,7:819-837.

23. Hosack DA, Dennis G, Jr., Sherman BT, Lane HC, Lempicki RA. Identifying biological themes within lists of genes with EASE. Genome Biol 2003;4:R70.

24. Campbell DR, Gross MD, Martini MC, Grandits GA, Slavin JL, Potter JD. Plasma carotenoids as biomarkers of vegetable and fruit intake, Cancer Epidemiol Biomarkers Prev 1994;3:493.500.

25. Kuefer R, Varambally S, Zhou M, Lucas PC, Loeftler M, Wolter H, Matteldt T, Hautmann RE, Gschwend JE, Barrette TR, Dunn RL, Chinnaiyan AM, Rubin MA. alpha-Methylacyl-CoA racemase: expression levels of this novel cancer biomarker depend on tumot differentiation. Am J Pathol 2002;161:841-8.

26. Carter $\mathrm{CA}$. Protein kinase $\mathrm{C}$ as a drug tanget implications for drug or diet prevention and treatment of cancer. Curr Drug Tangets 2000,1:163-83.

27. Kahl-Rainer P, Sedivy R, Marian B. Protein kinase C rissue localization in human colonic tumon suggests a role for adenoma growth control, Gastroenterology 1996;110:1753-9.

28. Zhao H, Watkins JL, Piwnica-Worms H. Disruption of the checkpoint kinase $1 /$ cell division cycle $25 \mathrm{~A}$ pathway abrogates ionizing radiation-induced S and G2 checkpoints. Proc Natl Acad Sci U S A 2002;99:14795-800.

29. Yasmeen A, Berdel WE, Serve H, Muller-Tidow C. E- and A-type cyclins as markers for cancer diagnosis and prognosis. Expert Rev Mol Diagn 2003;3:617-33.

30. Zhao L, Samuels T, Winckler S, Korgaonkar C, Tompkins V, Horne MC, Quelle DE. Cyclin G1 has growth inhibitory activity linked to the ARF-Mdm2-p53 and pRb tumor suppressor pathways. Mol Cancer Res 2003;1:195-206.

31. Momand J, Wu HH, Dasgupta G. MDM2-master regulator of the p53 tumor suppressor protein. Gene 2000;242:15-29,

32. Zheleva DI, Lane DP, Fischer PM. The p53-Mddm2 pathway: tangets for the development of new anticancer therapeutics. Mini Rev Med Chem 2003:3:257-70.

33. Stopera SA, Davie JR, Bird RP. Colonic aberrant crypt foci are associated with increased expression of c-fos: the possible role of modified c-fos expression in preneoplastic lesions in colon cancer. Carcinogenesis 1992;13:573-8.

34. Phillips RW, Kikendall JW, Luk GD, Willis SM, Murphy JR, Maydonovitch C, Bowen PE, Stacewicz-Sapuntzakis M, Wong RK. beta-Carotene inhibits rectal mucosal ornithine decarboxylase activity in colon cancer patients. Cancer Res 1993:53:3723-5.

35. Pai R, Soreghan B, Szabo II, Pavelka M, Baatar D, Tarnawski AS. Prostaglandin E2 transactivates EGF receptor: a novel mechanism for promoting colon cancer growth and gastrointestinal hypertrophy. Nat Med 2002;8:289-93.

36. Plummer SM, Holloway KA, Manson MM, Munks RJ, Kaptein A, Farrow S, Howells L_ Inhibition of cyclo-oxygenase 2 expression in colon cells by the chemopreventive agent curcumin involves inhibition of NF-kappaB activation via the NIK/IKK signalling complex. Oncogene 1999;18:6013. 20. 
37. Wenzel $\mathrm{U}$, Kuntz $\mathrm{S}$, Brendel $\mathrm{MD}$, Daniel $\mathrm{H}$. Dietary flavone is a potent apoptosis inducer in human colon carcinoma cells. Cancer Res 2000;60:3823-31.

38. Vincis P, Malats N, Lang A, d'Errico A, Caporaso N, Cuzick J, Boffetta P. Metabolic polymorphisms and susceptibility to cancer. IARC Scientific Publications No.148, 1999.

39. Kallay E, Adlercreutz H, Farhan H, Lechner D, Bajna E, Gerdenitsch W, Campbell M, Cross HS. Phytoestrogens regulate vitamin D metabolism in the mouse colon: relevance for colon tumor prevention and therapy. J Nutr 2002;132:3490S-3493S.

40. Martinez C, Garcia-Martin E, Ladero JM, Sastre J, Garcia-Gamito F, Diaz-Rubio M, Agundez JA. Association of CYP2C9 genotypes leading to high enzyme activity and colorectal cancer risk. Carcinogenesis 2001;22:1323-6.

41. Snyderwine EG, Turesky R), Turtelaub KW, Davis CD, Sadrich N, Schut HA, Nagao M, Sugimura T, Thorgeirsson UP, Adamson RH, Thotgeirsson SS. Metabolism of food-derived heterocyclic amines in nonhuman primates. Mutat Res 1997;376:203-10. 




\section{Chapter 3}

\section{Dose dependent effect of a vegetable mixture on gene expression in colon mucosa of C57BL6 female mice}

Submitted

S.G.J. van Breda ${ }^{1}$

E. van Agen ${ }^{1}$

S. van Sanden ${ }^{2}$

T. Burzykowski²

A.S. Kienhuis 1

J.C.S. Kleinjans ${ }^{1}$

J.H.M. van Delft'

'Department of Health Risk Analysis and Toxicology, Maastricht University, Maastricht, The Netherlands ${ }^{2}$ Center for Statistics, 1 imburg University Centre, Diepenbeck, Belgium 


\begin{abstract}
There is abundant epidemiological evidence that colorectal cancer (CRC) risk is decreased by consumption of vegetables. However, the molecular targets at the genome level are mostly unknown. The aim of the present study was to identify genes which are dose dependently modulated by vegetables, in order to provide more insight into the genetic mechanisms through which vegetables exert their beneficial effects. Female C57BL6 mice were randomly assigned to one of four dietary groups. Each group was fed one of four different diets, containing a $0 \%, 10 \%, 20 \%$ and $40 \%$ wt $/$ wt vegetables mixture respectively, for a period of two weeks. The vegetables mixture consisted of cauliflower ( $30 \%$ wet wt), carrots ( $30 \%$ wet wt), peas ( $30 \%$ wet wt), and onions ( $10 \%$ wet wt). In order to assess gene expression changes at target level, colonic mucosal cells were collected after sacrifice. Total RNA was isolated and microarray technology was used to measure the expression levels of 602 genes simultaneously. For 39 genes a significant dose-dependent effect was detected, although in general the relationship was not linear, 17 of these genes are likely to be involved in CRC protective mechanisms. For almost all of these genes, the

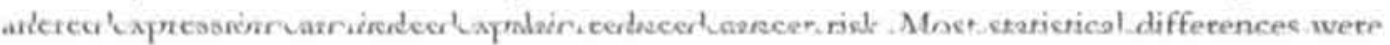
observed between the highest vegetable dose group $(40 \%)$ and one of the other diet groups. $\Lambda$ diet high in vegetables positively modulates genes hypothetically involved in protective mechanisms at various stages of CRC development. Next to genes which are already known to be induced by vegetables (components), new genes were identified which may play a role in CRC prevention by vegetables in humans.
\end{abstract}




\section{Introduction}

Colorectal cancer (CRC) is one of the most common cancers woridwide, with about 943,000 new cases per year.' Mortality has declined steadily, indicating the beneficial effects of improved therapy and early detection. However, incidence of this type of cancer is generally increasing, pointing out the need for additional preventive measures. ${ }^{2}$ It has been suggested that as much as $90 \%$ of CRC cases might be prevented by changes in diet. ${ }^{3}$ There is abundant epidemiological evidence that CRC development is strongly influenced by food and nutrition. Particularly, the consumption of diets high in vegetables, decreases the risk of CRC.4,5 The evidence from animal studies is less clear, but in the majority of these studies, it was found that vegetables or vegetable components reduce CRC. risk. ${ }^{\text {s.9 }}$

A large number of potentially anticarcinogenic substances is present in vegetables, which can influence the process of carcinogenesis via various mechanisms. For protection against CRC by fiber, which is present in all vegetables and is particularly high in pulses like beans and peas, proposed mechanisms comprise diluting and binding of carcinogens in the digestive tract, decreasing transit time of faecal bulk, inhibiting cell proliferation by diluting bile acids 10,11 and influencing indirectly the transcription rates of genes..$^{10,12}$ Allium vegetables, which comprise onions and garlic, contain organosulfur compounds like diallyl sulfide which can modify carcinogen activation by inhibition of phase I biotransformation enzymes $^{13}$ and induction of phase II detoxification enzymes. ${ }^{11 .} 14$ Other phytochemicals known to have an effect on the metabolic activation of procarcinogens, are isothiocyanates and indoles, breakdown products of glucosinolates present in cruciferous vegetables, which include cauliflower, broccoli, cabbage and Brussels sprouts: induction as well as inhibition of detoxification enzymes by these compounds has been described. ${ }^{15}$.11

Another important mechanism by which vegetables can protect against DNA damage, is scavenging of reactive oxygen species and other free radicals. Antioxidants like $\beta$ - and $\alpha$-carotene present in orange vegetables like carrots and pumpkin, are capable of protecting the DNA from free radical damage ${ }^{11,} 20$. Furthermore, these agents are able to suppress cell proliferation by upregulation of connexin 43, a gene responsible for maintaining intercellular gap junctional communication, which is associated with decreased proliferation. 20.21

In many of these postulated mechanisms, modulation of gene expression plays a role. Regulation of gene expression occurs by complex arrays of response elements that influence the rate of transcription and thereby regulate the abundance of proteins. Vegetable components can influence gene expression by direct interaction with regulatory elements in the genome, leading to a change in the transcription rate of a given gene. ${ }^{12}$

Gene expression modulation by vegetables and vegetable components has only been investigated in laboratory animals for a limited number of genes and often in relation 
to exposure of carcinogens. Many of the molecular targets at the genome level are unknown. In this study, the effect of different doses of a mixture of vegetables on gene expression in colon mucosa of female C57BL6 mice was investigated. This mouse model is frequently used in studies investigating dietary modulation at the molecular level, including gene expression, and it provides the basis of multiple transgenic mouse models, which can be used in future studies to investigate the specific role of a particular gene.6, 7. 22-26 Microarray technology was used to measure the expression levels of 602 genes simultaneously. Identification of genes dose-dependently modulated by vegetables can give more insight into the genetic mechanisms through which vegetables may exert their beneficial effects.

\section{Materials and Methods}

\section{Animals and Diet}

Eight-week-old female C57BL6 mice (Charles River Laboratories, Lyon, France) were randomly assigned to one of four dietary groups, each consisting of seven animals. During 7 days of acclimatization, all the animals received the standard control diet. Next, each group was fed one of four different diets, containing a $0 \%$ (referred to as control diet), $10 \%, 20 \%$ and $40 \% \mathrm{wt} / \mathrm{wt}$ vegetables mixture respectively, for a period of two weeks. The vegetables mixture used consisted of cauliflower (30\% wet wt), carrots $(30 \%$ wet wt), peas ( $30 \%$ wet wt), and onions ( $10 \%$ wet wt). Up to $20 \%$ vegetables were also used in previous mice vegetables studies, though no multiple vegetable dose diets were tested.9. 26. 27 Diets were refreshed every two days and provided as powdered feed. The $20 \%$ casein reference diet (Hope Farms, Woerden, The Netherlands) served as the basal diet for the four different diets. The vegetables were purchased as a single batch at the supermarket and separately cooked under household conditions. After freezing $\left(-20^{\circ} \mathrm{C}\right)$, the vegetables were lyophilized, ground and combined. Before the vegetables mixture was mixed with the basal diet, it was analyzed for macronutrient content (Hope Farms). The vegetables mixture added to the basal diet was at the expense of carbohydrates. Diets were adjusted for the amount of carbohydrates with dextrose/cerelose and cellulose (dicacel) (Hope Farms), resulting in similar energy densities. No antioxidants or preservatives were added. After preparation, the diets were stored at $-20^{\circ} \mathrm{C}$ in air-closed plastic bags until use. The composition of the different diets is presented in Table 1.

The animals were maintained in the laboratory animal care facilities at Maastricht University under controlled environmental conditions (temperature $21 \pm 1{ }^{\circ} \mathrm{C}$, relative humidity $50 \% \pm 10,12$-hr light/dark cycle). Body weights of the mice were recorded weekly. The study was approved by the Institutional Committee of Animal Experimentation Maastricht University. 
Table 1. Composition of the four diets in $\mathrm{g} / \mathrm{kg}$.

\begin{tabular}{|c|c|c|c|c|}
\hline Ingredients & Control diet ${ }^{1}$ & $\begin{array}{c}10 \% \text { Vegetable } \\
\text { diet }^{2}\end{array}$ & $\begin{array}{c}20 \% \text { Vegetable } \\
\text { diet }^{2}\end{array}$ & $\begin{array}{c}40 \% \text { Vegetable } \\
\text { diet }^{2}\end{array}$ \\
\hline Cerelose/Dextrose & 515 & 422 & 329 & 143 \\
\hline DL-Methionine & 2 & 2 & 2 & 2 \\
\hline $\mathrm{NaCl}$ & 3 & 3 & 3 & 3 \\
\hline Choline Cl. $50 \%$ & 4 & 4 & 4 & 4 \\
\hline Soyz oil & 50 & 50 & 50 & 50 \\
\hline $\mathrm{KCl}$ & 7 & 7 & 7 & 7 \\
\hline $\mathrm{KH}_{2} \mathrm{PO}_{4}$ & 7 & 7 & 7 & 7 \\
\hline $\mathrm{MgO}$ & 2 & 2 & 2 & 2 \\
\hline $\mathrm{C}_{2} \mathrm{HPO}_{4}, 2 \mathrm{H}_{2} \mathrm{O}$ & 13 & 13 & 13 & 13 \\
\hline $\mathrm{CaCO}_{3}$ & 10 & 10 & 10 & 10 \\
\hline Corn Starch & 100 & 100 & 100 & 100 \\
\hline Dicacel/Cellulose & 77 & 70 & 63 & 49 \\
\hline Cascin & 200 & 200 & 200 & 200 \\
\hline $\mathrm{MgSO}_{4}, 7 \mathrm{H}_{2} \mathrm{O}$ & 4 & 4 & 4 & 4 \\
\hline $\begin{array}{l}\text { Standard vitamin } \\
\text { premix }\end{array}$ & 3 & 3 & 3 & 3 \\
\hline $\begin{array}{l}\text { Standard } \\
\text { micronutrient premix }\end{array}$ & 3 & 3 & 3 & 3 \\
\hline Vegetables mixture' & 0 & 100 & 200 & 400 \\
\hline
\end{tabular}

IControl diet is the basal $20 \%$ casein reference diet.

In the vegetable diets, the basal diet was adjusted for cerelose/dextrose and dicacel/cellulose resultung in similar energy densities for all diets.

Composition ( $\%$ wet weight): cauliflower $(30 \%)$, carrots $(30 \%)$, peas $(30 \%)$ and onions $(10 \%)$,

\section{Tissue Sampling}

Mice were sacrificed by bleeding the vena cava inferior under Nembutal (Sanofi Sante, Maasluis, the Netherlands) anesthesia. Nembutal was administered subcutaneously in the neck at a dose of $60 \mathrm{mg} / \mathrm{kg}$ body weight. The large intestine was removed and placed on a specially made plastic box, which was kept at $4{ }^{\circ} \mathrm{C}$. After removing the rectum, the colon was opened longitudinally with fine scissors, and mucus and faeces were removed. Colonic mucosal cells were incubated in Trizol ${ }^{\mathrm{TM}}$ (GIBCO Life Technologies, Breda, The Netherlands) for 3 minutes and scraped off the muscle layer with the edge of a sterile glass slide. Cells were transferred into $800 \mu \mathrm{l}$ Trizol ${ }^{\mathrm{TM}}$, homogenized by resuspendation and stored at $-80^{\circ} \mathrm{C}$ until total RNA isolation.

\section{Total RNA Isolation and DNA Probe Synthesis}

Total RNA was extracted according to the manufacturer's instructions. The RNeasy® Mini Kit (Qiagen, Westburg, Leusden, The Netherlands) together with a DNase treatment was used to purify total RNA from salts and residual DNA. Quantity of each RNA sample was 
measured by a spectrophotometer and varied from 30 to $100 \mu \mathrm{g}$ per mouse. Integrity was determined by a Bioanalyzer (Agilent Technologies Netherlands B.V., Amsterdam, The Netherlands). All samples contained intact total RNA with an rRNA ratio $(28 \mathrm{~S} / 18 \mathrm{~S})>1.5$.

The $0 \%$ vegetable diet group was considered as the control group; the vegetable diet groups were referred to as the dose groups. Three total RNA pools per diet group were prepared by pooling equal amounts of total RNA from two or three mice. Cyanine 3(Cy3) and Cyanine 5- (Cy5) labeled cDNA probes were prepared using $10 \mu \mathrm{g}$ total RNA from each pool, by the method of Hasseman et al. ${ }^{28}$

\section{cDNA Micmarray Preparation}

This study is part of a project that investigates the effects of vegetables on gene expression in colon mucosa of humans and mice. For the human study, the expression levels of genes in colon mucosa were measured using the PHASE-1 Microarray Human-600 (PHASE-1 Molecular Toxicology, Santa fee, USA). A mouse cDNA microarray was constructed based on the genes present on the PHASE-1 Microarray Human-600. These genes represent a dedicated selection of biologically relevant gene sequences involved in inflammation, DNA damage and repair, oxidative stress, cell signaling, cell proliferation, metabolism, transcription and apoptosis.

In total, 602 mouse cDNA clones from the I.M.A.G.E. consortium were selected using Homologene of the National Center for Biotechnology Information (http://www.ncbi.nlm.nih.gov/HomoloGene/). The largest group of cDNAs consisted of 358 cDNA clones, originating from the National Institute on Aging (NIA, Bethesda, USA), and was provided by the Genome Centre Maastricht, The Netherlands. The remaining $244 \mathrm{cDNA}$ clones were obtained from the Resource Centre/Primary Database (RZPD, Berlin, Germany). All cDNA clones were provided as bacterial glycerol stocks and amplification of the CDNA inserts was performed by PCR in 96-well format in a Tgradient Thermocycler (Whatman Biometra, Westburg, Leusden, The Netherlands).

Amplification of the NIA cDNA inserts was carried out using $1.25 \mu \mathrm{l}$ bacterial glycerol stock as template in a $125 \mu \mathrm{l}$ reaction mixture consisting of $1 \mathrm{x}$ PCR buffer (Amersham Biosciences, Roosendaal, the Netherlands), $0.2 \mathrm{mM}$ dNTPs (Invitrogen Life Technologies, Breda, The Netherlands), victorase (Genome Centre, Maastricht University, Maastricht, The Netherlands) and $0.25 \mathrm{pmol} / \mu \mathrm{l}$ vector primers (15K-forward primer 5 'CCAGTCACGACGTTGTAAAACGAC-3'; and 15K-reverse primer 5'GTGTGGAATTGTGAGCGGATAACAA-3’; Eurogentec, Seraing, Belgium). Thermal cycling conditions consisted of an initial denaturation at $95^{\circ} \mathrm{C}$ for 3 minutes, followed by 35 cycles of $95^{\circ} \mathrm{C}$ for 45 seconds, $60^{\circ} \mathrm{C}$ for 45 seconds and $72{ }^{\circ} \mathrm{C}$ for 2 minutes.

To amplify cDNA inserts from the RZPD clones, two different primer sets were used. For amplification of the pT7T3D-pac1, pSPORT1, pCMV-SPORT2, Bluescribe and pBluescript SK vectors, M13-primers were used (M13-forward primer 5'- 
CCCAGTCACGACGTTCTAAAACG-3; and M13-reverse primer 5'. AGCGGATAACAATTTCACACAGG-3', Qiagen). PCR was performed in a $125 \mu \mathrm{l}$ reaction mixture containing $0.125 \mu \mathrm{l}$ bacterial glycerol stock, $10 \mathrm{mM}$ Tris- $\mathrm{HCl}(\mathrm{pH} 9.0), 50$ $\mathrm{mM} \mathrm{KCl}, 1.5 \mathrm{mM} \mathrm{MgCl}, 0.2 \mathrm{mM}$ dNTPs, $0.4 \mu \mathrm{M} \mathrm{M13}$ primers and $0.5 \mathrm{U}$ Taq DNA polymerase (Amersham Biosciences). Thermal cycling conditions consisted of an initial denaturation at $94^{\circ} \mathrm{C}$ for 10 minutes, followed by 30 cycles of $94{ }^{\circ} \mathrm{C}$ for 15 seconds, $56^{\circ} \mathrm{C}$ for 15 seconds and $72{ }^{\circ} \mathrm{C}$ for 45 seconds, and a final extension at $72{ }^{\circ} \mathrm{C}$ for 10 minutes. Sugano primers (Sugano-forward 5'-CTGCTCCTCAGTGGATGTTGCCTTTAC-3'; Sugano-reverse 5'-CAGGTTCAGGGGGAGGTGTGG-3'; Qiagen) were used to amplify the pME18S-FL3 vector. PCR was carried out in a $125 \mu \mathrm{l}$ reaction mixture containing $0.625 \mu \mathrm{l}$ bacterial glycerol stock, $10 \mathrm{mM}$ Tris- $\mathrm{HCl}$ ( $\mathrm{pH} 9.0$ ), $50 \mathrm{mM} \mathrm{KCl}, 4 \mathrm{mM} \mathrm{MgCl}, 0.2$ $\mathrm{mM}$ dNTPs, $0.2 \mu \mathrm{M} \mathrm{M13}$ primers and $2.5 \mathrm{U}$ Taq DNA polymerase (Amersham Biosciences). Thermal cycling conditions consisted of an initial denaturation at $95^{\circ} \mathrm{C}$ for 2 minutes, followed by 30 cycles of $95^{\circ} \mathrm{C}$ for 30 seconds, $52{ }^{\circ} \mathrm{C}$ for 30 seconds and $72{ }^{\circ} \mathrm{C}$ for 2 minutes, and a final extension at $72{ }^{\circ} \mathrm{C}$ for 5 minutes.

PCR products were verified by gel electrophoresis and purified using the QIAquick® 96-well purification kit (Qiagen). Purified PCR products were lyophilized and dissolved in 50\% DMSO in 384 well plates (Genetix, Hamphire, UK). Spotting of the PCR products onto UltraGAPSTM slides (Corning B.V. Life Sciences, Schiphol-Rijk, The Netherlands) was performed at the Genome Centre Maastricht using a pin-tool based spotting robot (Microgrid II, BioRobotics, Huntingdon, UK). DNA spots were arrayed in triplicate in order to reduce intra-assay variability in a $6 \times 8$ configuration. Printed slides were baked at $80^{\circ} \mathrm{C}$ for 3 hours and stored in a desiccator at room temperature until use.

\section{¿DNA Microarray Hybridizations}

For the hybridizations, within each diet group three pools were formed, each consisting of equal amounts of total RNA of two or three mice. By creating three pools per diet group instead of one, biological variability can be taken into account. This allows for a broader generalizability of the results. For each of the three sets of pools from different diet groups, a loop design was constructed with four microarrays, as follows: $\mathrm{DOj} \rightarrow \mathrm{D} 1 \mathrm{j} \rightarrow \mathrm{D} 2 \mathrm{j} \rightarrow \mathrm{D} 3 \mathrm{j} \rightarrow \mathrm{DOj}$ (the pools for control, $10 \%, 20 \%$ and $40 \%$ diet denoted, respectively, by $\mathrm{D} 0 \mathrm{j}, \mathrm{D} 1 \mathrm{j}, \mathrm{D} 2 \mathrm{j}$ and $\mathrm{D} 3 \mathrm{j}$ with $\mathrm{j}=1,2$ or 3 ; arrows join the samples put on the same array and indicate the sample labeled with $\mathrm{Cy}_{5}$ ). The loop is repeated three times, and then analyzed together, to reflect the possible variability in the experimental data. The statistical power of this design for estimating the dose-response profile is higher compared to the classical reference design in which each diet group is compared with the control group. Furthermore, fewer arrays are needed by using this design.29.31 In total 12 arrays were used. Cy 3 and $\mathrm{Cy} 5$ labeled cDNA probes of two groups were mixed according to this design and hybridized to the cDNA microarray by the method of Hasseman et al. 28 Slides 
were scanned on a GMS 418 Array Scanner (Affymetrix, High Wycombe, UK). The images obtained (resolution 10 micron; 16 bit tiff image) were processed with ImaGene 5.0 (Biodiscovery, LA,USA) to measure mean signal intensities for spots and local background.

\section{cDNA Microarray Data Processing and Statistical Analysis}

Dose-dependency was analyzed using ANOVA models. ${ }^{32}$ Following the suggestions of Kerr et al. (2000), no background-signal correction was applied, as it appeared to add extra unnecessary variability to the data. In the analysis, the base- 2 logarithmically transformed data were used. Due to computational limitations, the models were fit in two stages. The ANOVA model included a normalization step, taking into account both the global (acrossgenes) and local (gene-specific) normalization. 33

To investigate the differences in the gene expression levels, all pairwise differences between the treatment groups were examined. For each gene, the Tukey procedure was used to correct for multiple comparisons. ${ }^{34}$ To control the overall (across genes) probability of false positive findings at around $5 \%$, the procedure was applied using 0.0001 significance level per gene. Moreover, since the analysis of the fit of the model revealed possible problems with the assumption on the normality of the error terms, the critical values for the Tukey procedure were selected using an empirical distribution obtained by bootstrap rather than the theoretical distribution. ${ }^{35}$

\section{Real-time RT-PCR}

To verify the cDNA microarray results, 21 gene expression differences, representing six genes which were responsive to vegetables, were analyzed by real-time RT-PCR. First strand $\mathrm{CDNA}$ was generated according to the Superscript ${ }^{\mathrm{TM}}$ II RNase H Reverse Transcriptase protocol (Invitrogen, Life Technology) using $1 \mu \mathrm{g}$ total RNA. Forward and reverse primers for real-time PCR amplification were designed with Primer Express software version 1.5 (Applied Biosystem, Foster City, USA) using default settings. To avoid amplifying of contaminating genomic DNA, primers were chosen, either residing on different exons, thereby spanning at least 1 intron in the genomic sequence or on spanning the splicing junction site, therefore containing sequences from two adjacent exons. A list of validated genes and the normalization gene and their chosen primers is given in Table 2 . To normalize CDNA loading and PCR variations, the signals of the selected genes were corrected with the signals from $18 \mathrm{~S}$ mRNA as internal reference. ${ }^{36,37}$ Therefore, the amount of target gene is divided by the amount of $18 \mathrm{~S}$ of the sample to obtain a unit-less normalized target value.

PCR reactions consisted of $12.5 \mu \mathrm{l} 2 \mathrm{x}$ SYBR Green I qPCR Mastermix Plus, 0.75 $\mu \mathrm{l}$ SYBR Green I (Eurogentec), $2.5 \mu \mathrm{l} 3 \mu \mathrm{M}$ forward and reverse primer (Qiagen), $1.75 \mu \mathrm{l}$ milliQ water and $5 \mu \mathrm{l} \mathrm{cDNA}$ template (total $10 \mathrm{ng}$ template for validated genes; total $16 \mathrm{pg}$ template for the normalization gene 18S). 
Table 2. Primer sequences of the validated genes and the 185 normalization gene.

\begin{tabular}{|c|c|c|c|}
\hline $\mathbf{A C} \# \mathbf{I}$ & $\begin{array}{l}\text { Gene Name by NCBI } \\
\text { (Abbreviation) })^{2}\end{array}$ & Forward Primer & Reverse Primer \\
\hline $\mathrm{Al193731}$ & $\begin{array}{l}\text { Tumor necrosis factor receptor } \\
\text { superfamily,member } 6 \text { (TNFRSF6) }\end{array}$ & 5'-GCTGCGCCTCGTGTGAA-3' & 5'-GCGATTTCTGGGACTTTGTTTC-3' \\
\hline Al152753 & $\begin{array}{l}\text { Caspase 4, apoptosis-related } \\
\text { cysteine protease (CASP4) }\end{array}$ & 5'CGGGCAACCTTGACGAGAT-3' & 5'-TTCAAGCTGAAGAAATGAGATTCAGTT-3' \\
\hline $\begin{array}{l}\text { BG.063729, } \\
\text { B6,06377! }\end{array}$ & $\begin{array}{l}\text { Glycenaldehyde-3-phosphate } \\
\text { dehydrogenase (GAPDH) }\end{array}$ & $5^{\prime}-\mathrm{CCACCCACCCCAGCA \Lambda -3^{ \prime }}$ & 5'-AAATTGTGAGGGAGATGCTCAGT-3' \\
\hline A 1107664 & $\begin{array}{l}\text { Signal transducer and activator of } \\
\text { transcription } 1 \text { (STAT1) }\end{array}$ & 5'-GACCACGCCTTTGGGAAGTA-3' & 5:-TCGCTTAGGGTCGTCAAGCT-3' \\
\hline $\mathrm{A} 1226017$ & $\begin{array}{l}\text { Sulfotransferase family 1A, phenol- } \\
\text { preferring, member : (SULTLA1) }\end{array}$ & 5'-CCCTCAGAGTCTGCTGGATCA-3* & 5:-GGAGACAACCACATCCTTTGC-3' \\
\hline \multirow[t]{2}{*}{$\begin{array}{l}\text { BG.066160, } \\
\text { BG.074606 }\end{array}$} & Ornithine aminotransferase (OAT) & 5'-GTGAGAGGGAAAGGGTTGCTAA-3' & 5-CAGGCACACCTRCCAAGCAT-3' \\
\hline & 185 & 5-AGTCCCTGCCCTTTGTACACA-3 & 5-GATCCGAGGGCCTCACTAMAC-3: \\
\hline
\end{tabular}

AC is: GenRank accession numbers of the CDNA fragments ptesent in the microarrass

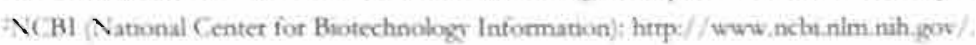


All PCR reactions were performed in triplicate in 96-well optical plates (Greiner Bio-One, Alphen a/d Rijn, The Netherlands) in the ABI Prism* 7700 Sequence Detection System. Data were analyzed using the ABI PRISM 7700 sequence detection system software version 1.9 .1 by means of the Relative Standard Curve Method.

\section{Additional Statistical Anatysis}

Statistical analysis of bodyweight of the mice was carried out using SPSS version 6.1.1 for Macintosh. Data were analyzed by means of analysis of variance followed by Student's ttest. A two-sided $\mathrm{p}$-value $<0.05$ was considered to indicate statistical significance.

\section{Results}

This study investigates the transcriptome differences induced in the colon of female C57BL6 mice fed one of four different diets, containing a $0 \%, 10 \%, 20 \%$ and $40 \%$ wt $/$ wt vegetables mixture respectively, for a period of two weeks. Mean body weights per week per group are presented in Table 3. During the week prior to the start of the intervention (week 0), all animals received the control diet. At the end of this week, there were no statistical significant differences in mean body weights between the groups. After the intervention, mean body weights were significantly increased within each group $(\mathrm{P}<0.05)$, but no significant differences between the groups were observed. Because weight gain was similar between the groups, these results indicate that there was no difference in food intake. Furthermore, these results show that the diets did not affect normal growth.

\section{Table 3. Mean body weights (g) per week per group.'}

\begin{tabular}{|lccc|}
\hline & Week 02 & Week 13 & Week 2 \\
\hline Control group & $18.6 \pm 0.6$ & $19.6 \pm 0.7$ & $20.3 \pm 0.7^{\mathbf{3}}$ \\
$10^{\circ} \%$ group & $18.6 \pm 1.3$ & $19.7 \pm 0.9$ & $20.2 \pm 0.8^{4}$ \\
$20^{\circ} \%$ group & $19.1 \pm 0.6$ & $20.0 \pm 0.4$ & $20.8 \pm 0.3^{4}$ \\
$40^{\circ} \%$ group & $18.6 \pm 0.4$ & $19.9 \pm 0.7$ & $20.6 \pm 1.0^{4}$ \\
\hline
\end{tabular}

'Body weights are expressed as mean \pm SF:

Acclimatization week, animals received control diet.

Intervention period.

'Statistics: analyses of vanance followed by Student's t-test. P<0.05: body weight significantly higher compared to week 0.

The expressions of 602 genes were measured simultaneously by means of cDNA microarrays. Microarray data analysis compared the levels of expression between the diet groups and tested whether these differences were significant. In total, a significant difference in gene expression was found for 96 diet group comparisons, representing 39 
genes. These genes, their matching comparisons and the differences are listed in Table 4. Remarkably, almost all the statistical differences are between the highest vegetable dose group $(40 \%)$ and one of the other diet groups. Biological functions and involved processes for the differentially expressed genes are diverse.

\section{Table 4. Differentially expressed genes.}

\begin{tabular}{|c|c|c|c|c|}
\hline Ac\#! & Gene Name by NCBI (Abbreviation)2 & Comparison & $\begin{array}{c}\text { Difference } \\
\text { cDNA } \\
\text { microarray }\end{array}$ & $\begin{array}{l}\text { Difference } \\
\text { RT-PCR }\end{array}$ \\
\hline BG073773 & $\begin{array}{l}\text { Translation clongation factor EF-1 alpha-1 } \\
\text { chain (EFHU1) }\end{array}$ & $40-0$ & $0.28 \pm 0.08$ & \\
\hline Al193731 & $\begin{array}{l}\text { Tumor necrosis factor receptor superfamily, } \\
\text { member } 6 \text { (TNFRSF6) }\end{array}$ & $\begin{array}{l}40-10 \\
40-20\end{array}$ & $\begin{array}{l}0.86 \pm 0.12 \\
0.89 \pm 0.10\end{array}$ & $\begin{array}{l}1.57 \pm 0.28 \\
1.12 \pm 0.07\end{array}$ \\
\hline w61433 & $\begin{array}{l}\text { SHB (Stc homology } 2 \text { domain containing) } \\
\text { adaptor protein B (SHB) }\end{array}$ & $\begin{array}{l}40-0 \\
40-10 \\
40-20\end{array}$ & $\begin{array}{l}0.64 \pm 0.11 \\
0.64 \pm 0.12 \\
0.74 \pm 0.11\end{array}$ & \\
\hline BC.064736 & $\begin{array}{l}\text { Ribonucleotide reductase MIl polypeptide } \\
\text { (RRM1) }\end{array}$ & $\begin{array}{l}40-0 \\
40-10 \\
40-20\end{array}$ & $\begin{array}{l}0.59 \pm 0.10 \\
0.60 \pm 0.12 \\
0.95 \pm 0.10\end{array}$ & \\
\hline BG063508 & MYB binding protein (P160) 1a (MYBBP1a) & $\begin{array}{l}40-0 \\
40-10\end{array}$ & $\begin{array}{l}-0.54 \pm 0.07 \\
-0.53 \pm 0.09\end{array}$ & \\
\hline BG067241 & $\begin{array}{l}\text { ATP-binding cassette, sub-family B } \\
\text { (MDR/TAP), member 1B (ABCB1b) }\end{array}$ & $\begin{array}{l}10-0 \\
20-10 \\
40-10\end{array}$ & $\begin{array}{r}1.17 \pm 0.11 \\
-0.59 \pm 0.10 \\
-1.10 \pm 0.11\end{array}$ & \\
\hline BG072904 & RAD51 homolog (S.cerevisiae) (RAD51) & $\begin{array}{l}40-0 \\
40-10 \\
40-20 \\
20-10\end{array}$ & $\begin{array}{r}0.61 \pm 0.13 \\
0.58 \pm 0.15 \\
0.86 \pm 0.13 \\
-0.29 \pm 0.13\end{array}$ & \\
\hline BG064900 & Stearoyl-Coenzyme A desaturase 2 (SCD2) & $\begin{array}{l}40-0 \\
40-20 \\
20-10\end{array}$ & $\begin{array}{r}0.54 \pm 0.10 \\
0.61 \pm 0.10 \\
-0.43 \pm 0.10\end{array}$ & \\
\hline BG064604 & Aldolase B, fructose-biphosphate (ALDOB) & $\begin{array}{l}40-10 \\
40-20\end{array}$ & $\begin{array}{l}-0.53 \pm 0.13 \\
-0.46 \pm 0.11\end{array}$ & \\
\hline BG063870 & $\begin{array}{l}\text { Beta actin, cytoplasmic (ACTB); clone } \\
\text { H3011G02 }\end{array}$ & $\begin{array}{l}40-0 \\
40-10 \\
40-20\end{array}$ & $\begin{array}{l}0.65 \pm 0.15 \\
0.75 \pm 0.17 \\
0.80 \pm 0.15\end{array}$ & \\
\hline BG064339 & $\begin{array}{l}\text { Beta actin, cytoplasmic (ACTB); clone } \\
\text { H3018D10 }\end{array}$ & $\begin{array}{l}40-0 \\
40-20\end{array}$ & $\begin{array}{l}0.65 \pm 0.15 \\
0.90 \pm 0.09\end{array}$ & \\
\hline Al152753 & $\begin{array}{l}\text { Caspase } 4 \text {, apoptosis-related cysteine protease } \\
\text { (CASP4) }\end{array}$ & $\begin{array}{l}40-10 \\
40-20 \\
20-0 \\
20-10 \\
10-0\end{array}$ & $\begin{array}{r}1.87 \pm 0.14 \\
1.28 \pm 0.12 \\
-0.78 \pm 0.14 \\
0.59 \pm 0.12 \\
-1.38 \pm 0.13\end{array}$ & $\begin{array}{r}2.78 \pm 0.13 \\
3.18 \pm 0.13 \\
-0.45 \pm 0.23 \\
0.40 \pm 0.13 \\
-0.05 \pm 0.23\end{array}$ \\
\hline
\end{tabular}


Table 4. (Continued)

\begin{tabular}{|c|c|c|c|c|}
\hline Ac\#1 & Gene Name by NCBI (Abbreviation) ${ }^{2}$ & Comparison & $\begin{array}{c}\text { Difference } \\
\text { cDNA } \\
\text { microarray }\end{array}$ & $\begin{array}{l}\text { Difference } \\
\text { RT-PCR }\end{array}$ \\
\hline \multirow[t]{3}{*}{ NM_0080841 } & Integrin beta I (fibronectin receptor beta) & $40-0$ & $0.72 \pm 0.09$ & \\
\hline & (ITGB1) & $40-10$ & $0.69 \pm 0.11$ & \\
\hline & & $40-20$ & $0.77 \pm 0.09$ & \\
\hline \multirow[t]{6}{*}{ AA796818 } & Interferon, alpha-inducible protein (G1P2) & $40-0$ & $3.25 \pm 0.13$ & \\
\hline & & $40-10$ & $2.01 \pm 0.16$ & \\
\hline & & $40-20$ & $4.24 \pm 0.13$ & \\
\hline & & $20-10$ & $-2.23 \pm 0.13$ & \\
\hline & & $20-0$ & $-0.99 \pm 0.16$ & \\
\hline & & $10-0$ & $1.24 \pm 0.14$ & \\
\hline \multirow[t]{3}{*}{ BG063771 } & Glyceraldehyde-3-phosphate dehydrogenase & $40-0$ & $0.71 \pm 0.09$ & $2.55 \pm 0.22$ \\
\hline & (GAPDH); clone H303E11 & $40-10$ & $0.55 \pm 0.11$ & $2.59 \pm 0.23$ \\
\hline & & $40-20$ & $0.65 \pm 0.09$ & $1.42 \pm 0.31$ \\
\hline \multirow[t]{3}{*}{186063729} & Glyceraldebyde-3-phosphate dehydrogenase & $40-0$ & $0.89 \pm 0.08$ & $2.55 \pm 0.22$ \\
\hline & (GAPDH); clone H3012A11 & $40-10$ & $0.74 \pm 0.10$ & $2.59 \pm 0.23$ \\
\hline & & $40-20$ & $0.75 \pm 0.08$ & $1.42 \pm 0.31$ \\
\hline \multirow[t]{3}{*}{ BC.066160 } & Ornithine aminotransferase (OAT); clone & $40-0$ & $-1.03 \pm 0.12$ & $-0.07 \pm 0.56$ \\
\hline & $\mathrm{H} 3039 \mathrm{~F} 05$ & $40-10$ & $-0.79 \pm 0.14$ & $-0.78 \pm 0.10$ \\
\hline & & $40-20$ & $-0.85 \pm 0.12$ & $-0.55 \pm 0.09$ \\
\hline \multirow[t]{2}{*}{ BG074606 } & Omithine aminotransferase (OAT); clone & $40-0$ & $-0.75 \pm 0.18$ & $-0.07 \pm 0.56$ \\
\hline & H3137B06 & $40-20$ & $-0.77 \pm 0.18$ & $-0.55 \pm 0.09$ \\
\hline \multirow[t]{3}{*}{ A1226017 } & Sulfotransferase family IA, phenol-preferring, & $40-0$ & $-1.28 \pm 0.11$ & $-1.29 \pm 0.16$ \\
\hline & member 1 (SULT1A1) & $40-10$ & $-1.62 \pm 0.12$ & $3.07 \pm 0.18$ \\
\hline & & $40-20$ & $-1.57 \pm 0.11$ & $3.69 \pm 0.16$ \\
\hline BG,072707 & $\begin{array}{l}\text { Spermidine/spermine N1-acetyl transferase } 1 \\
\text { (SAT) }\end{array}$ & $40-20$ & $0.63 \pm 0.12$ & \\
\hline \multirow[t]{3}{*}{ BG063081 } & Thymosin, beta 10 (TMSB10) & $40-0$ & $0.54 \pm 0.12$ & \\
\hline & & $40-10$ & $0.63 \pm 0.14$ & \\
\hline & & $40-20$ & $0.80 \pm 0.12$ & \\
\hline \multirow[t]{5}{*}{ AN107664 } & Signal transducer and activator of transcription & $40-0$ & $1.45 \pm 0.13$ & $3.91 \pm 0.25$ \\
\hline & 1 (STAT1) & $40-10$ & $1.39 \pm 0.15$ & $2.81 \pm 0.34$ \\
\hline & & $40-20$ & $2.48 \pm 0.13$ & $4.17 \pm 0.11$ \\
\hline & & $20-0$ & $-1.04 \pm 0.15$ & $-0.26 \pm 0.25$ \\
\hline & & $20-10$ & $-1.10 \pm 0.13$ & $-4.17 \pm 0.34$ \\
\hline \multirow[t]{3}{*}{ AA798640 } & Tryptophan hydroxylase 1 (TPH) & $40-20$ & $-1.18 \pm 0.12$ & \\
\hline & & $20-0$ & $0.86 \pm 0.13$ & \\
\hline & & $20-10$ & $0.69 \pm 0.11$ & \\
\hline BG065392 & Cathepsin B (CTSB) & $40-20$ & $0.46 \pm 0.07$ & \\
\hline \multirow[t]{2}{*}{ A1119367 } & Caspase 7 (CASP7) & $40-10$ & $0.73 \pm 0.11$ & \\
\hline & & $40-20$ & $0.73 \pm 0.09$ & \\
\hline
\end{tabular}


Table 4. (Continued)

\begin{tabular}{|c|c|c|c|c|}
\hline Ac\#1 & Gene Name by NCBI (Abbreviation) ${ }^{2}$ & Comparison & $\begin{array}{c}\text { Difference } \\
\text { cDNA } \\
\text { microarray }^{3}\end{array}$ & $\begin{array}{l}\text { Difference } \\
\text { RT-PCR }\end{array}$ \\
\hline BG07956-4 & Leukemia inhibitory factor (L.IF) & $40-20$ & $-0.49 \pm 0.07$ & \\
\hline \multirow[t]{3}{*}{ BGon 5853} & Sclenoprotein P, plasma 1 (SEPP1) & $40-0$ & $-1.10 \pm 0.14$ & \\
\hline & & $40-10$ & $-1.18 \pm 0.16$ & \\
\hline & & $40-20$ & $-1.07 \pm 0.14$ & \\
\hline AA500809 & Peripheral myelin protein (PMP22) & $40-0$ & $0.80 \pm 0.12$ & \\
\hline BG066650 & $\begin{array}{l}\text { Proteasome (prosome, macropain) } 28 \text { subunit, } \\
\text { alpha (PSME1) }\end{array}$ & $40-20$ & $0.92 \pm 0.14$ & \\
\hline \multirow[t]{2}{*}{ BCO65380 } & Pyruvate kinase, muscle (PKM2) & $40-0$ & $0.62 \pm 0.07$ & \\
\hline & & $40-20$ & $0.59 \pm 0.07$ & \\
\hline BGon3104 & Ribosomal protein S17 (RPS17) & $40-0$ & $-0.50 \pm 0.09$ & \\
\hline \multirow[t]{2}{*}{$A 1256565$} & Hydroxyprostaglandin dehydrogenase 15 & $40-10$ & $-0.91 \pm 0.16$ & \\
\hline & (NAD) (HPGD) & $40-20$ & $-0.66 \pm 0.14$ & \\
\hline BG071325 & Calbindin 3 (CALB3) & $40-20$ & $-2.68 \pm 0.23$ & \\
\hline An157359 & P311 protein (P311) & $40-20$ & $0.93 \pm 0.14$ & \\
\hline BG,064747 & Glutamic-pyruvate transaminase (GPT) & $10-0$ & $0.93 \pm 0.14$ & \\
\hline \multirow[t]{2}{*}{ BG086656 } & Aldehyde dehydrogenase family 1 , subfamily A1 & $40-0$ & $0.96 \pm 0.12$ & \\
\hline & (ALDH1A1) & $10-0$ & $1.39 \pm 0.13$ & \\
\hline AA759852 & Solute carrier family 26, member 3 (SLC.26A3) & $40-0$ & $1.31 \pm 0.18$ & \\
\hline \multirow[t]{2}{*}{ BG070529 } & Caspase 3, apoptosis related cysteine protease & $40-0$ & $0.76 \pm 0.12$ & \\
\hline & (CASP3) & $40-20$ & $0.85 \pm 0.12$ & \\
\hline AA510124 & Chemokine (C-X-C motif) ligand 10 (CXCL10) & $40 \cdot 20$ & $1.28 \pm 0.18$ & \\
\hline W29265 & $\begin{array}{l}\text { Glutathione S-transferase, alpha } 2 \text { (Yc2) } \\
\text { (GSTA2) }\end{array}$ & $40-20$ & $1.38 \pm 0.12$ & \\
\hline \multirow[t]{2}{*}{ BG064142 } & Hypoxia inducible factor 1 , alpha subunit & $40-0$ & $1.33 \pm 0.15$ & \\
\hline & (HIF1A) & $40-20$ & $1.11 \pm 0.15$ & \\
\hline \multirow{2}{*}{$\begin{array}{c}\text { BG064535 } \\
-\end{array}$} & Squalene epoxidase (SQLE) & $40-0$ & $0.83 \pm 0.10$ & \\
\hline & & $40-20$ & $0.90 \pm 0.10$ & \\
\hline
\end{tabular}

'AC\#: GenBank accession numbers of the CDNA fragments present on the microarrays.

NCBI (National Center for Biotechnology Informanon): http://www.ncbt.nim.nih.gow/.

'Estimated difference in mean 7 log-transformed intensiry \pm SE.

Normalized difference in mean Fog-transformed intensiry \pm SE. Twenty one gene differences were validated with RT-PC.R. Empry cells indicate gene differences which were not taken into sceount.

At least 17 genes are, according to literature review, likely to play a role at different stages during CRC development and are discussed in more detail. They are involved in biotransformation [Sulfotransferase family 1A, phenol-preferring, member 1 (SULT1A1), Glutathione S-transferase, alpha 2 (Yc2) (GSTA2), Hydroxyprostaglandin dehydrogenase 15 (NAD) (HPGD), Aldehyde dehydrogenase family 1, subfamily A1 (ALDH1A1)], apoptosis [Tumor necrosis factor receptor superfamily, member 6 (TNFRSF6), Caspase 4, apoptosis-related cysteine protease (CASP4), Caspase 7 (CASP7), Caspase 3, apoptosis 
related cysteine protease (CASP3), cathepsin B (CTSB), Thymosin beta 10 (TMSB10), Signal transducer and activator of transcription 1 (STAT1)], DNA repair [RAD51 homolog (S.cerevisiae) (RAD51)], polyamine metabolism [Ornithine aminotransferase (OAT), Spermidine/spermine N1-acetyl transferase 1 (SAT)], and tumor suppression [ribonucleotide reductase M1 polypeptide (RRM1), Solute carrier family 26, member 3 (SIC.26A3), MYB binding protein (P160) 1a (MYBBP1a)].

In Figure 1, the estimated differences per vegetable diet group compared to the $0 \%$ vegetable diet group (set to 0 ) of these genes are depicted. This figure shows that most effects of the vegetables on gene expression occur in the highest vegetable dose group, and that in general dose-response relationship was no clear linear. For the expression of the genes SULF1A1, RAD51, TMSB10, STAT1, OAT and RRM1 the effect of vegetables in the highest dose group is significantly different from the expression in the other vegetable groups. This seems also true for GSTA2, HPGD, TNFRSF6, CASP3, CASP4, CASP7, CTSB, SAT, and MYB, although not all differences between the highest vegetable dose group and the other dose groups are statistically significant. These results indicate that there is a relatively high dose of vegetables necessary to exert a measurable effect on colonic gene expression. The only exceptions to this are the ALDH1A1 and SLC26A3 genes, For these genes the effect on expression is similar in all three vegetable groups.

SULT1A1

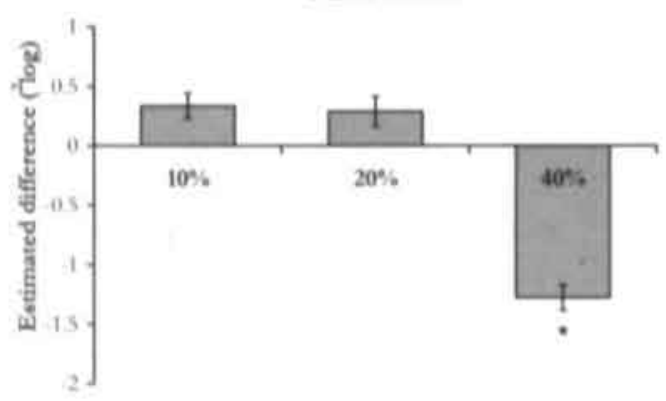

GSTA2

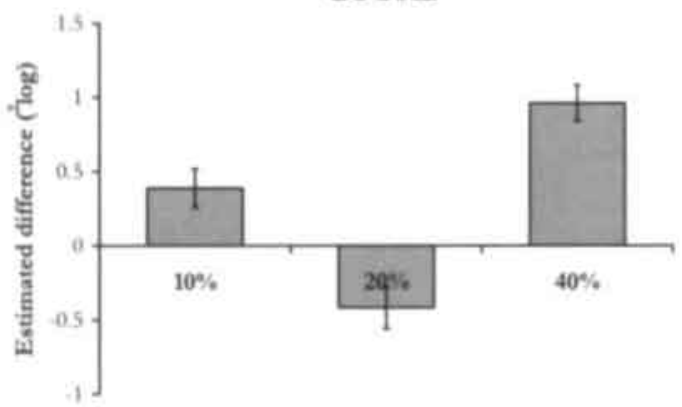

Figure 1. Eistmated difference in mean Jog-transformed intensiry \pm SE per diet group ( $x$-axis) for the genes that are tikely ro play a role at differen stages durng. CRC development. The $O^{\circ}$. diet intensity (set to i) serves as the reference. Astensk indicates significant differences between diet group and control ( $0^{2}$ - diet group). SU1.TIAI: Sulforransferase famaly IA. phenol preferring, member 1; GSTA2 Glutathione S-transferase, alpha 2 (Yc2): HPGD: Hydroxyprostaglandin dehydrogenase 15 (NAD); ALDH1A1: Aldehyde dehydrogenase family 1, subfamily A1: R.AD51: R.D51 homolog (S.cerevisac); TNFRSF6: Tumor necrosis factor receptor superfamly. member 6; C.ASP3: Caspase 3, apoptosts related cysteine protease: CASP7; Caspase 7: CASP4: Caspase 4, apoptosis-related cysteune protease: CTSB: Cathepsun B: TMISB10: Thymosin beta 10, STAT1: Signal transducet and activator of transcrpption 1; SAT: Spermudine/spermane N1-aceryl transferase 1; ONT: Oruthine aminotransferase, RRM11: Ribonucleotede reductase MII polypeptede; S1.C26.A3, Solute carrict family 26, member 3; C.MMB, C.myb proto-oncogene. See Table 4 for other stanstical detals. 
HPGD

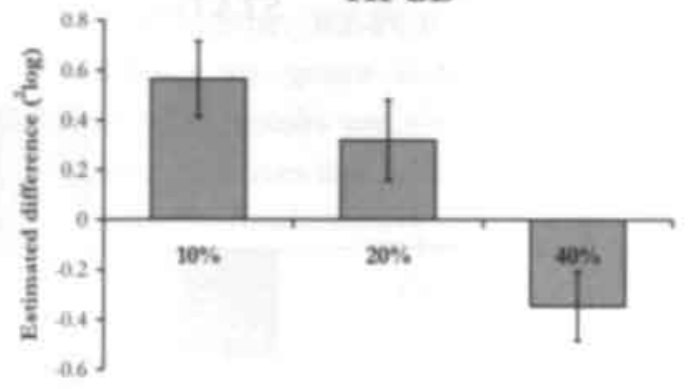

RAD51

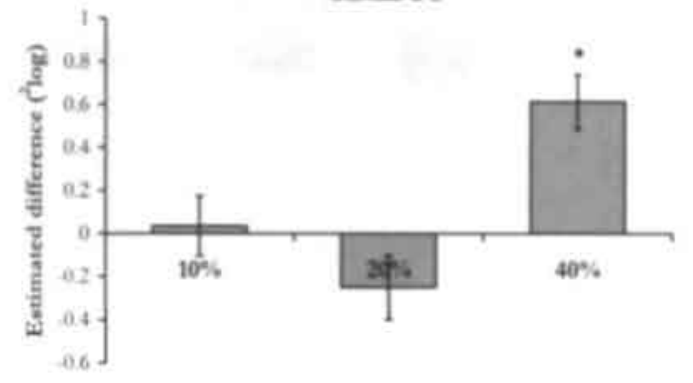

CASP3

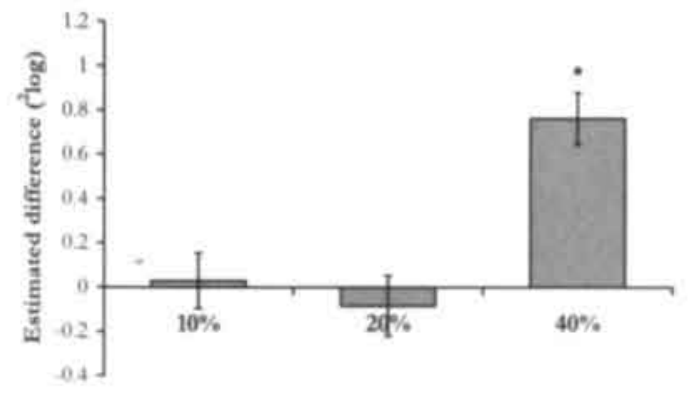

CASP4

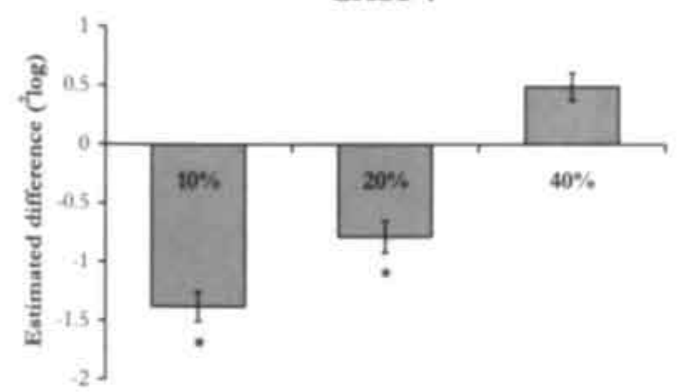

ALDH1A1

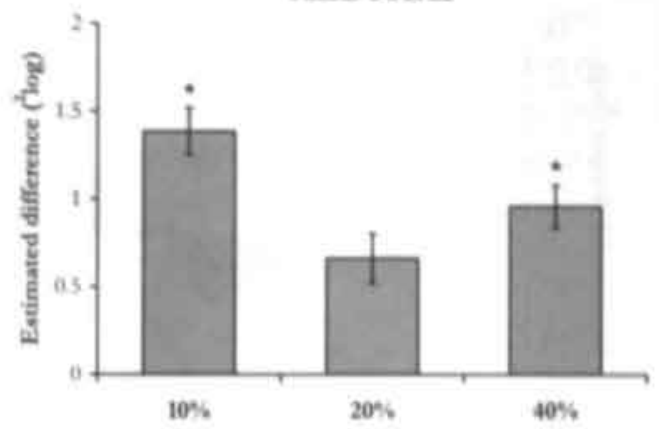

TNFRSF6

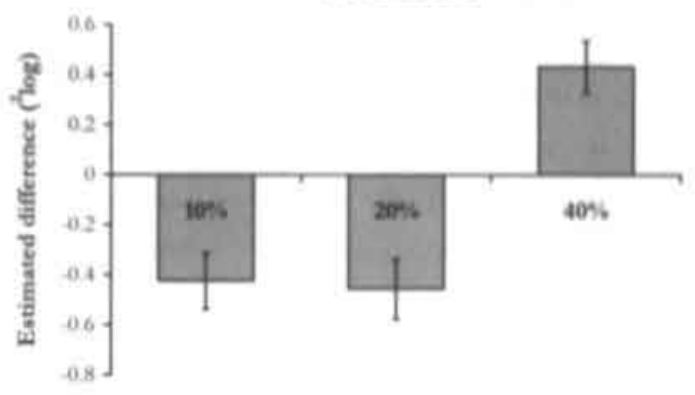

CASP7

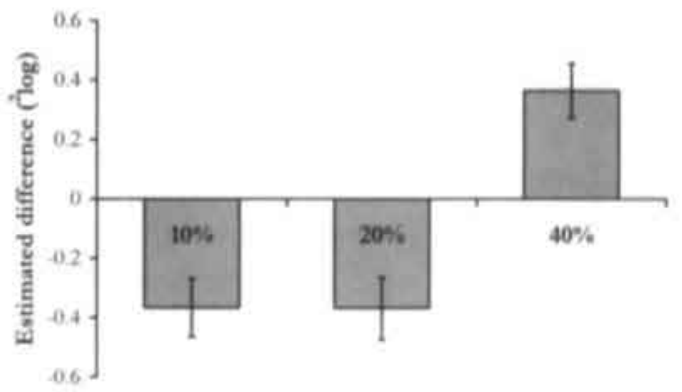

CTSB

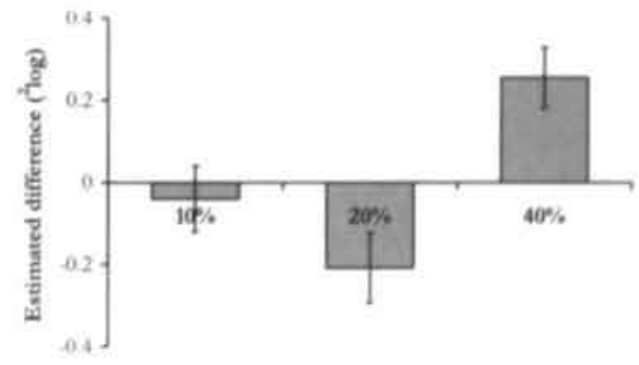

Figure 1. (Continued) 
TMSB10

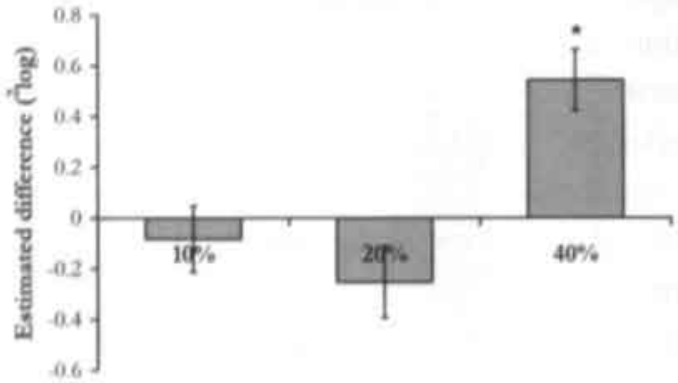

SAT

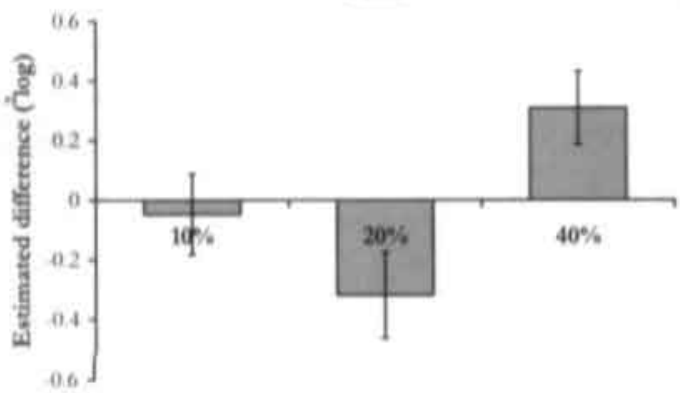

OAT; clone H3137B06

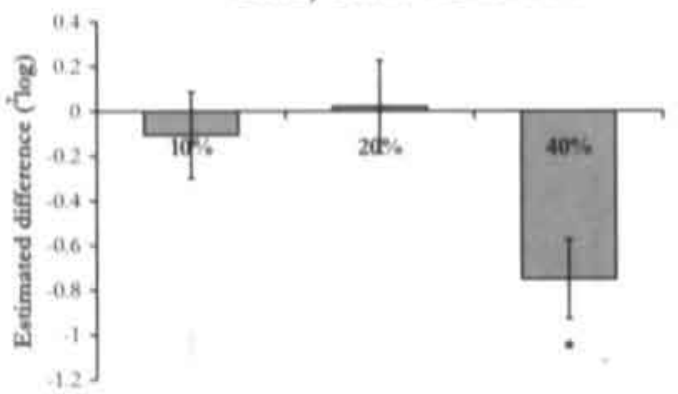

SLC26A.3

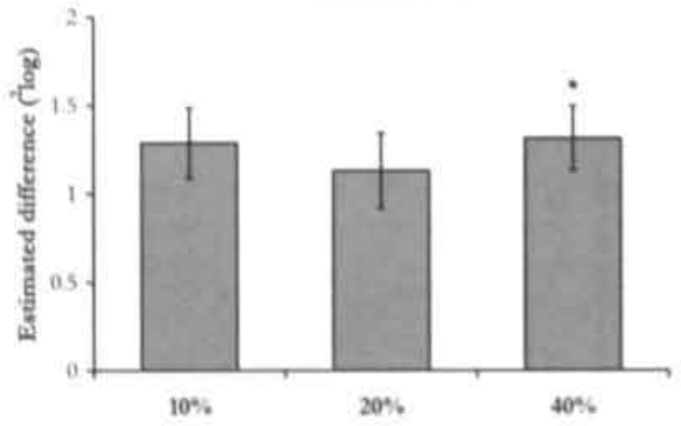

STAT1

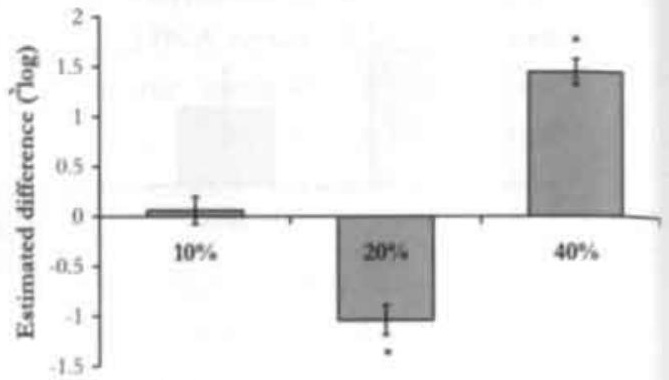

OAT; clone H3039F05

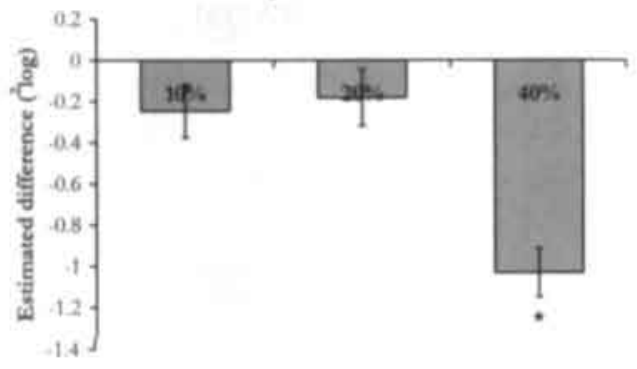

RRM1

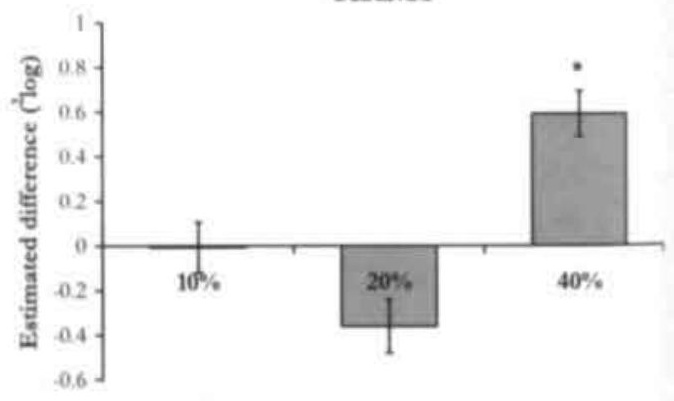

C-MYB

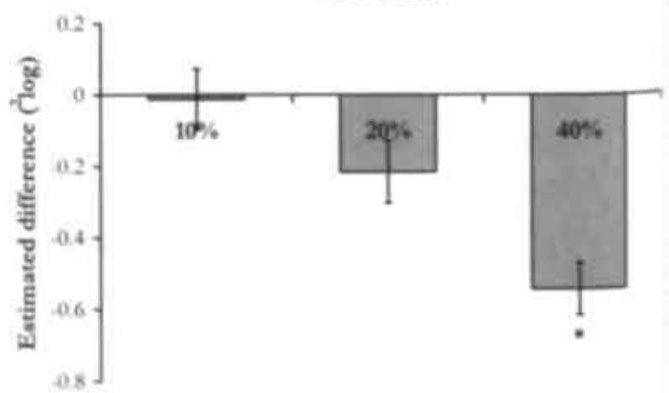

Figure 1. (Continued) 
Real-time RT-PCR was used to validate 21 gene expression differences, representing six genes responsive to vegetables, as identified by CDNA microarray analyses. The results are shown in Table 4 . These data confirm the results obtained by microarray analyses and verify the reliability of this cDNA microarray approach to identify genes that are modulated by vegetables.

\section{Discussion}

In the present study, a cDNA microarray approach was used to analyze the dosedependent changes in gene expression in response to diets supplied with different doses of a mixture of vegetables in colon mucosa of mice, in order to provide insight into the mechanisms underlying CRC prevention by vegetables.

CRC develops as the result of the progressive accumulation of genetic and epigenetic alterations that lead to the transformation of normal colonic epithelium into colon adenocarcinoma, as presented in the genetic model by Vogelstein. ${ }^{30}$ At each of the presented stages, various genetic mechanisms are possible by which vegetables and/or vegetable components can inhibit progression to the next stage.

The results of this study show a dose-dependent effect of vegetables on gene expression, although in general the relationship was not linear. At least 17 genes which may play a role at different stages during CRC development were significantly modulated at this dose. Table 5 summarizes the effects on gene expression in relation to possible effects on carcinogenesis.

The first line of defense against initiation of CRC is the ability of the colon tissue to intercept and detoxify potentially DNA damaging xenobiotic or endogenous substances. In this study, three genes involved in xenobiotic metabolism were affected, i.e. SULT1A1, GSTA2 and ALDH1A1. SULT1A1 and GSTA2 encode both for phase-II biotransformation enzymes, but these enzymes differ in their mode of action. Sulfotransferase enzymes catalyze the sulfate conjugation of many hormones, neurotransmitters, drugs and xenobiotic compounds and play an important role in detoxification of these compounds. However, they also play an important role in the metabolism and bioactivation of many dietary and environmental mutagens, including heterocyclic aromatic amines (HCA) implicated in the pathogenesis of colorectal and other cancers. ${ }^{39},{ }^{40}$ Several food components have already been shown to be potent inhibitors of SULT1A1 like quercetin ${ }^{41,42}$, grape juice ${ }^{42}$ and curcumin ${ }^{43}$. In contrast to SULT1A1, the GSTA2 enzyme is able to detoxify HCA, next to a number of other electrophilic carcinogenic metabolites like benzo(a)pyrene-7,8-dihydrodiol-9,10-epoxide and aflatoxin B1-8,9-oxide ${ }^{44}$, by coupling to glutathion. ${ }^{45}$ In addition to this major activity, GST's from 
the alpha class, like GSTA2, are known to catalyze glutathione peroxidase reactions and are therefore an essential component of the cellular antioxidant defense mechanism. ${ }^{46}$ The GST enzymes are inducible by drugs, carcinogens, antioxidants and other dietary components. 4,47 In this study, the expression of SULT1A1 was inhibited in the highest dose group, compared to the other diet groups, whereas the expression of GSTA2 was increased in the highest dose group. Next to these effects on phase-II biotransformation genes, vegetables induced another detoxifying gene, i.e. ALDH1A1. This gene encodes for an enzyme which belongs to the family of several isoenzymes involved in the metabolism of exogenous and endogenous aldehydes. This particular isoenzyme is the next enzyme after alcohol dehydrogenase in the major pathway of alcohol metabolism. It oxidizes acetaldehyde to acetetic acid and thereby protects cells from the adverse effects of acetaldehyde. There is increasing evidence that acetaldehyde is highly toxic, mutagenic and carcinogenic. $^{48}$ In this study, the expression of ALDH1A1 was significantly higher in the $10 \%$ and $40 \%$ vegetable groups compared to the control group. The effects of vegetables on the expression of SULT1A1, GSTA2 and ALDH1A1 support the model that high vegetable consumption results in decreased colon cancer risk, by inhibition of formation and detoxification of colon carcinogens.

Table 5. Presentation of differentially expressed genes known to be involved in (colon) carcinogenesis, the direction of the effect in the $40 \%$ vegetable group compared to control, the theoretical effect of this on cancer risk, and the involved pathways.

\begin{tabular}{|c|c|c|c|}
\hline $\begin{array}{l}\text { Gene Name by } \\
\text { NCBI } \\
\text { (Abbreviation)' }\end{array}$ & $\begin{array}{l}\text { Effect on expression in the } \\
\text { highest }(40 \%) \text { vegetable } \\
\text { group compared to control }\end{array}$ & $\begin{array}{c}\text { Theoretical effect on } \\
\text { cancer risk }\end{array}$ & Involved pathway \\
\hline SULTIAI & - & - & Biotransformation \\
\hline GSTA2 & + & - & \\
\hline HPGD & - & - & \\
\hline ALDHIAI & + & - & \\
\hline RAD51 & + & - & DNA repair \\
\hline TNIRSF 6 & + & - & Apoptosis \\
\hline CASP4 & + & - & \\
\hline CASP7 & + & - & \\
\hline CASP3 & + & - & \\
\hline CTSB & + & - & \\
\hline TMSBIO & + & - & \\
\hline STATI & + & - & \\
\hline OAT & - & + & Polyamine \\
\hline SAT & + & - & metabolism \\
\hline RRMII & + & - & Tumor invasion \\
\hline SI.C.26A3 & + & - & \\
\hline MYBBPla & - & + & \\
\hline
\end{tabular}

'NCBI (National Center fot Botechnology Information); hrtps//www.ncbinlm.nih.gov/. 
A gene involved in generation of DNA reactive compounds is the HPGD gene, which was down-regulated in the highest vegetable group. It encodes for an enzyme which metabolizes a number of prostaglandins and non-prostanoid compounds. The products of the non-prostanoids compounds are generally highly reactive $\alpha, \beta$-unsaturated aldehydes and ketones, which may cause carcinogenesis. HPGD is a target for many non-steriodal anti-inflammatory drugs (NSAIDs), including cyclooxygenase-2 inhibitors, which have been shown to decrease the risk of colon cancer in both epidemiological and experimental studies. Also phytophenolic compounds such as curcumin, flavonoids and epigallocatechin gallate were found to inhibit HPGD enzyme activity, $4 \%$ so The mechanism of chemoprevention by these compounds could be by inhibition of HPGD mRNA resulting in a diminished HPGD enzyme activity, leading to a decreased production of the highly reactive $\alpha, \beta$-unsaturated aldehydes and ketones in the colon tissues. Down-regulation of HPGD in the highest vegetable group indicates the presence of vegetable (components) acting as HGPD inhibitors.

If a genotoxic reaction with DN $\Lambda$ has occurred, cancer initiation can still be prevented by repairing this lesion. One DNA repair gene was up-regulated, namely RAD51. This gene encodes for the key enzyme for homologous recombination which is an evolutionarily conserved mechanism for the repair of double strand breaks and for generation of genetic diversity. Disruption of the RAD51 gene leads to an increased number of chromosome aberrations, including chromosome breaks and finally in cellular lethality, suggesting that this gene is important for maintenance genome stability, ${ }^{51}, 52$ Vegetables as inducers of this DNA repair gene have not previously been described, but in this study up-regulation of this gene was observed in the highest vegetable group compared to the other diet groups and suggests that vegetables could protect cells from DNA damage by increasing the DNA repair capacity.

- Apoptosis (programmed cell death) is a common process in the intestinal crypt, by removing differentiated cells which form the surface epithelium. In the stem cell and proliferation zone of the colorectal crypt, apoptosis occurs to a lesser extent. Here, genetically damaged stem cells are removed before they can undergo clonal expansion. Stimulation of apoptosis by vegetables provides a protective mechanism against CRC, by eliminating genetically damaged cells before they can transform to tumor cells. Seven genes known to be involved in apoptosis were affected by vegetables in this study. Apoptosis is mediated via cell surface receptors (APO1/CD95 and the tumor necrosis factor (TNF) receptor family) and the mitochondria. Activation of the cell surface receptor by an extracellular ligand causes an intracellular region, the death effector domain, to form a cytosolic complex with pro-caspase- 8 , followed by a release of caspase- 8 , which initiates downstream activation of effector caspases 353,454 and 7.33 The activation of these caspases results in programmed cell death. ${ }^{53}$ TNFRSF6, CASP4, CASP7, and CASP3 were up-regulated in the highest vegetable dose group. CTSB can trigger the mitochondrial 
pathway of apoptosis. This protease is released from lysosomes into the cytosol during intracellular cytotoxic-signaling cascades and thereby increasing the cytochrome $c$ release from mitochondria..$^{53}$ Cytochrome $c$ forms a protein complex with the apoptosis activating factor-1 that recruits procaspase 9, resulting in its activation. Caspase 9 activates caspase 3 , initiating a caspase cascade that leads to cell death. 53,56 CTSB can also trigger apoptosis in a caspase-independent pathway, whereby CTSB functions as the dominant execution protease..$^{57}$ This mechanism could represent a backup program in tumor cells, because here the standard apoptosis pathway is frequently impaired and the expression of CTSB is increased. However, CTSB has another but opposing role in malignancy, namely enhancing the invasiveness of the tumor cells. 58 TMSB10, a small actin-binding protein, has also dual functions: programmed cell death ${ }^{59}$ and tumor invasion ${ }^{60}$ The seventh gene affected by vegetables which is involved in apoptosis is STAT1. The mechanism by which STAT1 induces apoptosis is not clear, but may involve indirect up-regulation of Fas and caspase activation. ${ }^{61}$ In the colon mucosa of mice receiving the highest vegetable dose diet the TNFRSF6, CASP4, CASP7, CASP3, CTSB, TMSB10 and STAT1 genes were upregulated and could thereby provide a protective mechanism against neoplasia by removing through apoptosis genetically damaged stem cells before they can proliferate. ${ }^{53}$

Next to genes involved in apoptosis, genes concerned with cell proliferation and invasion where modulated by vegetables. Two genes involved in polyamine metabolism were modulated. Polyamines are ubiquitous cellular polycations essential for optimal rates of cell growth and differentiation. ${ }^{62}$ Increased polyamine content has been shown to correlate with higher CRC risk. Mutations in the adenomatous polyposis coli gene results in higher polyamine content in the intestinal mucosa ${ }^{63.65} \mathrm{SAT}$, up-regulated in the highest vegetable group, is the key enzyme in polyamine catabolism ${ }^{64}$, causing polyamine contents to drop. NSAIDs are able to activate SATs and are already used as chemopreventive agents for colon carcinogenesis ${ }^{64} \mathrm{OAT}$, down-regulated in the highest vegetable group, is also involved in polyamine metabolism. The protein-product of this gene converses ornithine to glutamate semialdehyde, thereby reducing intracellular ornithine contents. Ornithine is the substrate of ornithine decarboxylase (ODC), which is the rate-limiting enzyme in polyamine synthesis. ${ }^{66} \mathrm{~A}$ decrease in OAT activity could contribute to an increase in ornithine available for ODC, leading to increased polyamine synthesis. The result of the effect of vegetables on polyamine metabolism and subsequent cell growth is not clear and has to be further investigated.

Several genes involved in tumor suppression were modulated by vegetables. Until now, no relation between vegetables and expression has previously been described for one of these genes, i.e the RRM1 gene, which was up-regulated in the highest vegetable group. This gene encodes for the large subunit (M1) of ribonucleotide reductase, the heterodimeric enzymes that catalyzes the rate-limiting step in deoxyribonucleotide synthesis, providing a balanced supply of precursors for DNA synthesis and repair. ${ }^{67}$ Aside 
from this role, RRM1 functions as a metastasis suppressor gene by suppressing invasion, migration and in trivo metastasis formation through upregulation of the phosphatase and tensin homolog (PTEN) tumor-suppressor gene, when overexpressed in human and mouse cancer cell lines. ${ }^{68}$ SLC26A3 or DRA (down-regulated in adenoma) is an important tumor-suppressor gene associated with CRC risk and was up-regulated in the highest vegetable group compared to the control group. This gene is expressed exclusively in normal colon tissue ${ }^{69}$, and it encodes a membrane specific anion transporter ${ }^{70}$ for transporting sulfate and chloride into the cell. ${ }^{71}, 72$ Next to this, it induces growthsuppression in colon cells, what correlates with inhibition of colon tumor progression. ${ }^{\text {t3 }}$ Up-regulation of this colon tumor suppressor gene by vegetables provides another mechanism by which vegetables can protect against colon cancer risk by inducing growth inhibition. However, next to this beneficial effect on the DRA tumor suppressor gene, the MYBBP1a gene was down-regulated in the highest vegetable group, which inhibits the cmyb proto-oncogene (C-MYB) protein. C-MYB is predominantly expressed in immature hemopoietic cells where it plays an important role in hemopoietic cell proliferation and differentiation. ${ }^{74} \mathrm{C}$-MYBb expression is restricted to just a few tissues and the role in these tissues is less clear. MYBBP1a is ubiquitously expressed in human and mouse cell lines and tissues and therefore it is thought that this gene has a broader cellular function than only being a myb-interacting protein. ${ }^{75}$ The result of this modulation in relation to colon cancer development remains unclear and needs to be further investigated.

Although there is a gap between gene expression and CRC risk, it is found that vegetables exert an effect on intermediate biomarkers for CRC risk such as aberrant crypt foci and intestinal polyps. The aberrant crypt foci are considered as precursor lesions that would progress to overt CRC. In a study of Rijken et al (1999), rats fed different diets supplemented with peas, spinach, sprouts or a mix of different vegetables experienced a reduction in aberrant crypt foci (ACF) multiplicity. ${ }^{9}$ Furthermore, Kim et al (2003) investigated the effect of indole-3-carbinol (constituent of cruciferous vegetables) supplemented diets on $\mathrm{ACF}$ in $\mathrm{C} 57 \mathrm{BL} / 6 \mathrm{j}$ mice. Total number of $\mathrm{ACF}$ was significantly decreased in these animals compared to animals which received the standard diet. Next to an effect on ACF, a reduction of intestinal polyp development was observed in $\mathrm{C} 57 \mathrm{BL} / 6 \mathrm{j}$ apcin/+ mice fed the indole-3-carbinol supplemented diets. ${ }^{6}$ Mahmoud et al (2000) also reported a decrease in intestinal polyp development in C57BL/6j-ap $c^{\mathrm{Mtin} / *}$ mice fed diets supplemented with the plant phenolics caffeic acid phenethyl ester or curcumin.

In this study, the dose of vegetables necessary to exert a measurable effect on mouse colonic gene expression is relatively high, i.e. $40 \%$ of the diet. In the human population (females, age range 22-65 years), approximately $20 \%$ of the total daily food intake comprise vegetables and fruits, accounting for $270 \mathrm{~g}(\sim 150 \mathrm{~g}$ vegetables and $\sim 120 \mathrm{~g}$ fruits). ${ }^{76}$ In this respect, it can be stated that on average, hurnans probably consume not enough vegetables and fruit to activate cancer preventive pathways. This is in line with the 
latest recommendation made by The World Health Organization, which advises an intake of at least $400 \mathrm{~g}$ of vegetables and fruits (accounting for approximately $30 \%$ of the total daily food intake) to reduce the risk of cancer of the gastrointestinal tract. ${ }^{77}$

In summary, the results of this study show a dose-dependent effect of vegetables on gene expression changes in the colon mucosa of female mice. Genes were modulated which are involved in protective mechanisms at the various stages of CRC development. A diet with a high content of vegetables modulates genes involved in inhibition of carcinogen formation, increasing DNA repair capacity, induction of apoptosis, and in reducing cell growth and -tumor invasion. Next to genes which are already known to be induced by vegetables (components), new genes were identified, i.e. the detoxification gene ALDH1A1, the DNA repair gene RAD51 and the apoptosis genes TNFRSF6, CASP4, CASP7, CASP3, CTSB, TMSB10 and STAT1, the metastases suppressor gene RRM1 and the tumor suppressor gene SLC26A3. These genes may play an important role in CRC prevention by vegetables and may provide new molecular targets for CRC prevention. The possible importance of these genes and their modulation by vegetables has to be further investigated. 


\section{References}

1. World Health Organization \& International Agency for Research on Cancer. World Cancer Report. Lyon. LARCPress, 2003.

2 Greenwald P. Colon cancer overview, Cancer 1992;70:1206-15.

3. Doll R, Peto $R$. The causes of cancer: quantitative estimates of avoidable risks of cancer in the United States today. J Nat Cancer Inst 1981;66:1191-308.

4. World Cancer Research Fund. Food, Nutrition and the Prevention of Cancer: a Global Perspective. Chapter 4.10: Colon, rectum. Washington: BANTA Book group, 1997:216-251.

5. Steinmetz KA, Potter JD. Vegetables, fruit, and cancer prevention: a review. J Am Diet Assoe 1996;96:1027-39.

6. Kim DJ, Shin DH, Ahn B, Kang JS, Narn KT, Park CB, Kim CK, Hong, JT, Kim YB, Yun YW, Jang DD, Yang KH. Chemoprevention of colon cancer by Korean food plant components. Mutat Res 2003:523-524:99-107.

7. Mahmoud NN, Carothers AM, Grunberger D, Bilinski RT, Churchill MR, Martucd C, Newmark HI. Bertagnolli MM. Plant phenolics decrease intestinal tumors in an animal model of familial adenomatous polyposis. Carcinogenesis 2000;21:921-7.

8. Kallay E, Adlercreutz H, Farhan H, Lechner D, Bajna E, Gerdenitsch W, Campbell M, Cross HS. Phytoestrogens Regulate Vitamin D Metabolism in the Mouse Colon: Relevance for Colon Tumor Prevention and Therapy. J Nutr 2002;132:3490S-3493S.

9. Rijken PJ, Timmer WG, van de Kooij AJ, van Benschop IM, Wiseman SA, Mejiers M, Tijburg LB. Effect of vegetable and carotenoid consumption on aberrant crypt multiplicity, a surrogate end-point marker for colorectal cancer in azoxymethane-induced rats, Carcinogenesis 1999;20:2267-72.

10. Lupton JR, Turner ND. Potential protective mechanisms of wheat bran fiber. Am I Med 1999;106:24S-27S.

11. Steinmetz KA, Potter JD. Vegetables, fruit, and cancer. II. Mechanisms. Cancer Causes Control 1991:2:427-42.

12. Cousins RJ. Nutritional regulation of gene expression. Am J Med 1999;106:20S-23S; discussion 50S515 .

13. Wargovich MJ. Experimental evidence for cancer preventive elements in foods. Cancer Lett 1997;114:11-7.

14. Guyonnet D, Siess MH, Le Bon AM, Suscheter M. Modulation of phase II enzymes by otganosulfur compounds from allium vegetables in rat tissues. Toxicol Appl Pharmacol 1999:154:50-8.

15. Wortelboer HM, de Kruif CA, van lersel AA, Noordhoek J, Blaauboer BJ, van Bladeren PJ, Falke HE. Effects of cooked brussels sprouts on cytochrome P.450 profile and phase II enzymes in liver and small intestinal mucosa of the rat. Food Chem Toxicol 1992;30:17-27.

16. Larsen-Su S. Williams DE. Dietary indole-3-carbinol inhibits FMO activity and the expression of flavin-containing monooxygenase form 1 in rat liver and intestine. Drug Metab Dispos 1996:24:92731.

17. Katchamart S, Williams DE. Indole-3-carbinol modulation of hepatic monooxygenases CYP1A1, CYPIA2 and FMO1 in guinea pig, mouse and rabbit. Comp Biochem Physiol C Toxicol Pharmacol 2001:129:377-84.

18. Sorensen M, Jensen BR, Poulsen HE, Deng X, Tygstrup N, Dalhoff K, Loft S. Effects of a Brussels sprouts extract on oxidative DNA damage and metabolising enzymes in rat liver. Food Chem Toxicol 2001:39:533-40. 
19. Clapper MI, Szarka CE, Pfeiffer GR, Graham TA, Balshem AM, Litwin S, Goosenbery EB, Frucht $\mathrm{H}$, Engstrom PF. Preclinical and clinical evaluation of broccoli supplements as inducers of glutathione S-transferase activity. Clin Cancer Res 1997;3:25-30.

20. Bertram JS, Bortkiewic $\mathrm{H}$. Dietary carotenoids inhibit neoplastic transformation and modulate gene expression in mouse and human cells. Am J Clin Nutr 1995;62:1327S-1336S.

21. Bertram JS. Carotenoids and gene regulation. Nutr Rev 1999;57:182-91.

22. Bates MD, Erwin CR, Sanford LP, Wiginton D, Bezerna JA, Schatzman LC, Jegga AG, Ley-Ebert C, Williams SS, Steinbrecher KA, Warner BW, Cohen MB, Aronow BJ. Novel genes and functional relationships in the adult mouse gastrointestinal tract identified by microarray analysis. Gastroenterology 2002;122;1467-82.

23. Kallay E, Adlercreutz H, Farhan H, Lechner D, Baina E, Gerdenitsch W, Campbell M, Cross HS, Phytoestrogens regulate vitamin D metabolism in the mouse colon: relevance for colon tumor prevention and therapy. J Nutr 2002;132:3490S-3493S.

24. Kawai J, Shinagawa A, Shibata K, Yoshino M, Itoh M, Ishii Y, Arakawa T, Hara A, Fukunishi Y, Konno H, Adachi J, Fukuda S, Aizawa K, Izawa M, Nishi K, Kiyosawa H, Kondo S, Yamanaka I, Saito T, Okazaki Y, Gojobori T, Bono H, Kasukawa T, Saito R, Kadota K, Matsuda H, Ashburner M, Batalov S, Casavant T, Fleischmann W, Gaasteriand T, Gissi C, King B, Kochiwa H, Kuchl P, I.ewis S, Matsuo Y, Nikaido I, Pesole G, Quackenbush J, Schriml LM, Staubli F, Suzuki R, Tomita M, Wagner L, Washio T, Sakai K, Okido T, Furuno M, Aono H, Baldarelli R, Barsh G, Blake J. Boffelli D, Bojunga N, Carninci P, de Bonaldo MF, Brownstein Mf, Bult C, Fletcher C, Fujita M, Gariboldi M, Gustincich S, Hill D, Hofmann M, Hume DA, Kamiya M, Lee NH, Lyons P, Marchionni 1., Mashima J, Mazzarelli J, Mombacrts P, Nordone P, Ring B, Ringwald M, Rodriguez I, Sakamoto N, Sasaki H, Sato K, Schonbach C, Seya T, Shibata Y, Storch KI, Suzuki H, Toyo-oka K, Wang KH, Weitz C, Whittaker C, Wilming L, Wynshaw-Boris A, Yoshida K, Hasegawa Y, Kawaji H. Kohtsuki S, Hayashizaki Y. Functional annotation of a full-length mouse cDNA collection. Nature 2001;409:685-90.

25. Orner GA, Dashwood WM, Blum CA, Diaz GD, LA Q, Dashwood RH. Suppression of tumorigenesis in the $A \mathrm{pc}(\mathrm{min})$ mouse; down-regulation of beta-catenin signaling by a combination of tea plus sulindac. Carcinogenesis 2003;24:263-7.

26. van Kranen HJ, van lersel PW, Rijnkels JM, Beems DB, Alink GM, van Kreijl CF. Effects of dietary fat and a vegetable-fruit mixture on the development of intestinal neoplasia in the ApcMin mouse. Carcinogenesis 1998:19:1597-601.

27. Rinkels JM, Nlink GiM. Effects of a vegetables-fruit mixture on liver and colonic 1,2. dimethylhydrazine-metabolizing enzyme activities in rats fed low- or high-fat diets, Cancer Lett 1998:128:171.5.

28. Hasseman J, Chen E, Yang 1, Quackenbush J. Aminoallyl labeling of RNA for microarrays. hrtp://www.tigr.ong/tdb/micoarray/protocolsTiGR.shtml (Accessed June 21, 2001), 2001.

29. Ketr MK. Design considenatons for efficient and effective microarray studies. Biometrics 2003,59:822-828.

30. Dobbin K. Shit JH, Simon R. Questions and answers on design of dual label microarrays for identifyung differentially expressed genes. I Nati Cancer Inst 2003;95:1362-9.

31. Churchill GA. Fundamentals of experimental design for cDNA microarrays. Nat Genet 2002;32 Suppl 2:490-5.

32. Kerr MK, Martin M, Churchill GA. Analysis of variance for gene expression microartay data. Journal of Computational Biology 2000;7:819-837. 
33. Wolfinger RD, Gibson G, Wolfinger ED, Bennett L, Hamadeh H, Bushel P, Afshari C, Paules RS. Assessing gene significance from cDNA microarnay expression data via mixed models. I Comput Biol 2001;8:625-37.

34. Neter J, Kutner MH, Nachtsheim CJ. Wasserman W. Applied Linear Statistical Models (Fourth Edition). Mass, 1996.

35. Kerr MK, Afshari CA, Bennett L. Bushel P. Martinez J, N.J. W, Churchill GA. Statistical analysis of a gene expression microarray experiment with replication. Statistica Sinica 2002;12:203-217.

36. Bustin SA. Absolute quantification of mRNA using real-time reverse transcription polymerase chain reaction assays. J Mol Endocrinol 2000;25:169-93.

37. Aerts J1, Gonzales MI. Topalian SI. Selection of approptiate control genes to assess expression of tumor antigens using real-time RT-PCR. Biotechniques 2004;36:84-6, 88, 90-1.

38. Fearon ER, Vogeistein B. A genetic model for colorectal tumorigenesis. Cell 1990,61:759-67.

39. Gamage NU, Duggieby RG, Barnett AC, Tresillian M, Latham CF, Ly̨ou NE, McManus ME, Martin JL. Structure of a human carcinogen-converting enzyme, SULT1A1. Structural and kinetic implications of substrate inhibition. J Biol Chem 2003;278:7655-62.

40. Wong CF, Liyou N, Leggert B, Young J, Johnson A, MeManus ME. Association of the SULT1A1 R213H polymorphism with colorectal cancer. Clin Exp Pharmacol Physiol 2002;29:754-8.

41. De Santi C. Pietrabissa A, Mosca F, Rane A. Pacifici GM. Inhibition of phenol sulfotransferase (SULT1A1) by quercetin in human adult and foetal livers. Xenobiotica 2002:32:363-8.

Tamura H, Matsui M. Inhibitory effects of green tea and grape juice on the phenol sulfotransferase activity of mouse intestines and human colon carcinoma cell line, Caco-2. Biol Pharm Bull 2000;23:695-9.

43. Vietri M, Pietrabissa A, Mosca F, Spisni R, Pacifici GM. Curcumin is a potent inhibitor of phenol sulfotransferase (SUIT1A1) in human liver and extrahepatic tissues. Xenobiotica 2003;33:357-63.

44. Morel F, Schulz WA, Sies H. Gene structure and regulation of expression of human glutathione Stransferases alpha. Biol Chem Hoppe Seyler 1994;375:641-9.

45. Coles B, Nowell SA, Macleod SI, Sweeney C, Lang NP, Kadlubar FF. The role of human glutathione S-transferases (hGSTs) in the detoxification of the food-derived carcinogen metabolite $\mathrm{N}$-acetoxy-PhIP, and the effect of a polymorphism in hGSTAI on colorectal cancer risk. Mutat Res 2001;482:3-10.

46. Yang Y, Sharma R, Zimniak P, Awasthi YC. Role of alpha class glutathione S-transferases as antioxidant enzymes in rodent tissues. Toxicol Appl Pharmacol 2002;182:105-15.

47. t Hoen PA, Rooseboom M, Bijsterbosch MK, van Berkel T], Vermeulen NP, Commandeur JN. Induction of giutathione-S-transferase mRNA levels by chemopreventive selenocysteine $\mathrm{Se}$ conjugates. Biochem Pharmacol 2002;63:1843-9.

48. Seitz HK, Matsuzaki S, Yokoyama A, Homann N, Vakevainen S, Wang XD. Alcohol and cancer. Alcohol Clin Exp Res 2001;25:137S-143S.

49. Cho H, Tai HH. Inhibition of NAD+-dependent 15-hydroxyprostaglandin dehydrogenase (15$\mathrm{PGDH}$ ) by cyclooxygenase inhibitors and chemopreventive agents. Prostaglandins 1 eukot Essent Fatty Acids 2002;67:461-5.

50. Cho H, Tai HH. Thiazolidinediones as a novel class of $\mathrm{NAD}(+)$-dependent 15-hydroxyprostaglandin dehydrogenase inhibitors. Arch Biochem Biophys 2002;405:247-51.

51. Stark JM, Hu P, Pierce AJ. Moynahan ME, Ellis N, Jasin M. ATP hydrolysis by mammalian RAD51 has a key role during homology-directed DNA repair. J Biol Chem 2002:277:20185-94. 
52. Kanamoto T, Hellman U, Heldin CH, Souchelnytskyi S. Functional proteomics of transforming growth factor-betal-stimulated Mv1Lu epithelial cells: Rad51 as a target of TGFbetal-dependent regulation of DNA repair. Embo J 2002;21:1219-30.

53. Johnson IT. Anticarcinogenic effects of diet-related apoptosis in the colorectal mucosa. Food Chem Toxicol 2002;40:1171-8.

54. Kamada S, Washida M, Hasegawa J, Kusano H, Funahashi Y, Tsujimoto Y. Involvement of caspase4(-like) protease in Fas-mediated apoptotic pathway, Oncogene 1997;15:285-90.

55. Guicciardi ME, Deussing J, Miyoshi H, Bronk SF, Svingen PA, Peters C, Kaufmann SH, Gores GJ. Cathepsin B contributes to TNF-alpha-mediated hepatocyte apoptosis by promoting mitochondrial release of cytochrome c. J Clin Invest 2000;106:1127-37.

56. Bradham CA, Qian T, Streetz K, Trautwein C, Brenner DA, Lemasters II. The mitochondrial permeability transition is required for tumor necrosis factor alpha-mediated apoptosis and cytochrome c release. Mol Cell Biol 1998,18:6353-64.

57. Foghsgaard L, Wissing D, Mauch D, Lademann U, Bastholm L, Boes M, Elling F, Leist M, Jaattela M. Cathepsin B acts as a dominant execution protease in tumor cell apoptosis induced by tumor necrosis factor. J Cell Biol 2001;153:999-1010.

58. Emmert-Buck MR, Roth MJ, Zhuang Z, Campo E, Rozhin J, Sloane BF, Liotta LA, StetlerStevenson WG. Increased gelatinase A (MMP-2) and cathepsin B activity in invasive tumor regions of human colon cancer samples. Am J Pathol 1994;145:1285-90.

59. Hall AK. Thymosin beta-10 accelerates apoptosis. Cell Mol Biol Res 1995;41:167-80.

60. Santelli G, Califano D, Chiappetta G, Vento MT, Bartoli PC, Zullo F, Trapasso F, Viglietto G, Fusco A. Thymosin beta-10 gene overexpression is a general event in human carcinogenesis. Am J Pathol 1999;155:799-804.

61. Xu X, Fu XY, Plate J, Chong AS. IFN-gamma induces cell growth inhibition by Fas-mediated apoptosis: requirement of STAT1 protein for up-regulation of Fas and FasL expression. Cancer Res 1998;58:2832.7.

62. Pegg AF. Polyamine metabolism and its importance in neoplastic growth and a target for chemotherapy. Cancer Res 1988:48:759:74.

63. Martinez ME, O'Brien TG, Fultz KE, Babbar N, Yerushalmi H, Qu N, Guo Y, Boorman D, Einspahr J, Alberts DS, Gerner EW. Pronounced reduction in adenoma recurrence associated with aspirin use and a polymorphism in the ornithine decarboxylase gene. Proc Natl Acad Sci U S A 2003:100:7859-64.

64. Babbar N, Gerner EW. Polyamines as modifiers of genetic risk factors in human intestinal cancers. Biochem Soc Trans 2003:31:388-92.

65. Wang W, Liu LQ. Higuchi CM. Mucosal polyamine measurements and colorectal cancer risk. J Cell Biochem 1996;63:252.7.

66. Han X, Kazaninoff MN, Seiler N, Stanley BA. Rat colon omithine and arginine metabolism: coordinated effects after proliferative stimuli. Am J Physiol Gastrointest Liver Physiol 2001;280:G3389-99.

67. Wright JA, Chan AK, Choy BK. Hurta RA, McClarty GA. Tagger AY. Regulation and drug resistance mechanisms of mammalian ribonucleotide reductase, and the significance to DNA synthesis. Biochem Cell Biol 1990,68:1364-71.

68. Gautam A, Li ZR, Bepler G, RRMI-induced metastasis suppression through PTEN-regulated pathways, Oncogene 2003;22:2135-42. 
69. Schweinfest CW, Henderson KW, Suster S, Kondoh N, Papas TS. Identification of a colon mucosa gene that is down-rogulated in colon adenomas and adenocarcinomas. Proc Natl Acad Sci U S A 1993;90:4166-70.

70. Byeon MK, Westerman MA, Maroulakou IG, Henderson KW, Suster S, Zhang XK, Papas TS, Vesely J, Wallingharn MC, Green JE, Schweinfest CW. The down-regulated in adenoma (DRA) gene encodes an intestine-specific membrane glycoprotein. Oncogene 1996;12:387-96.

71. Schultheis PJ, Clarke L., Meneton P, Miller MI. Soleimani M, Gawenis L.R, Riddle TM, Duffy IJ. Doetschman T, Wang T, Giebisch G, Aronson PS, Lotenz JN, Shull GE. Renal and intestinal absorptive defects in mice lacking the NHE $3 \mathrm{Na}+/ \mathrm{H}+$ exchanger. Nat Genet 1998;19:282-5.

72. Rajendran VM, Binder $\mathrm{HJ}$. $\mathrm{C}-\mathrm{HCO} 3$ and $\mathrm{Cl}-\mathrm{OH}$ exchanges mediate $\mathrm{Cl}$ uptake in apical membrane vesicles of rat distal colon. Am J Physiol 1993;264:G874-9.

73. Antalis TM, Reeder JA, Gotiey DC, Byeon MK, Walsh MD, Henderson KW, Papas TS, Schweinfest CW. Down-regulation of the down-regulated in adenoma (DRA) gene correlates with colon tumor progression. Clin Cancer Res 1998;4:1857-63.

74. Mucenski MI, Melain K, Kier AB, Swerdlow SH, Schreiner CM, Miller TA, Pietryga DW, Scott W]. Jr., Potter SS. A functional c-myb gene is required for normal murine fetal hepatic hematopoiesis. Cell 1991;65:677-89.

75. Keough R, Woollatt E, Crawfond J. Sutherland GR, Plummer S, Casey G, Gonda T]. Molecular doning and chromosomal mapping of the human homologue of MYB binding protein (P160) $1 \mathrm{~A}$ (MYBBP1A) to 17p13.3. Genomics 1999:62:483-9.

76. Anonymous. Zo eet Nederland. Resultaten van de voedselconsumpticpeiling 1997-1998. Voedingscentrum Den Haag, 1998.

77. Diet, nutrition and the prevention of chronic diseases. World Health Organ Tech Rep Ser 2003;916:i-viii, 1-149, backcover. 



\section{Chapter 4}

\section{Identification of proteins differentially expressed in colon mucosa of C57BL6 female mice by altered vegetable intake}

In Preperation

G. Breikers'

S.G.J, van Breda'

F.G. Bouwman ${ }^{2}$

M.H.M. Van Herwijnen'

J.W. Renes ${ }^{2}$

E.C.M. Mariman ${ }^{2}$

J.C.S. Kleinjans ${ }^{1}$

J.H.M. van Delft'

'Department of Health Risk Analysis and Toxicology, Maastricht University, Maastricht, The Netherlands 2Department of Human Biology, Maastricht University, Masstricht, The Netherlands 


\begin{abstract}
Colorectal cancer is one of the most common types of cancer in the world. Over $70 \%$ of the cases might be prevented by changes in diet and life style. Vegetable consumption is thought to have a protective effect against colorectal cancer and a positive relationship has already been found in both mouse and human studies. Until now, not much is known about the targets of preventive actions by vegetables at the genome and proteome level. In the current study, the effect of increased vegetable intake on the protein expression in the colonic mucosa of healthy mice was studied in order to identify the proteins of which the levels are affected by increased vegetable consumption. Mice were fed four different diets, containing respectively $0 \%, 10 \%, 20 \%$ or $40 \%$ of vegetables, and total cellular protein from colonic mucosal cells was analyzed by means of $2 \mathrm{D}$-gelelectrophoresis. We found 30 proteins that were differentially expressed in one or more vegetable groups as compared to the control group. Six of these proteins could be identified by MALDI-TOF mass spectrometry; namely MLRN, CAH-1, HMG-1, PAP3, GAPDH and OSCP. Except for OSCP, alterations in the levels of these proteins coincide with a pivotal role in the protection against colon cancer. We therefore conclude that the observed altered protein levels following increased vegetable intake, provide functional support for the protective effects of vegetables against colorectal cancer.
\end{abstract}




\section{Introduction}

Colorectal cancer (CRC) is one of the most common types of cancer in the Western world. Although mortality has declined, the incidence is generally increasing and preventive measures are therefore needed. 'The etiology of CRC is complex and involves both genetic and environmental factors. Known risk factors include a positive family history, age, meat and alcohol consumption and fat intake. It is thought that over $70 \%$ of CRC cases might be preventable by modification of the diet and life style risk factors ${ }^{2}$ and inverse associations are reported with physical activity, vegetable intake, and, although less consistent, with fruit consumption. ${ }^{1.5}$

Vegetables contain a large number of potentially anticarcinogenic compounds that can affect the process of carcinogenesis via various mechanisms. Using mice and rats, many studies have been performed investigating the role of individual vegetable derived compounds in the protection against CRC. The majority shows a protective effect of vegetable components against $\mathrm{CRC}^{6-8}$

Vegetables can exert their protective effect against CRC via several mechanisms like, for example, free radical scavenging, modulation of enzyme levels and transcriptional regulation. So far, the effect of vegetable consumption on gene and protein levels has not yet been extensively studied and the targets at the genome and proteome level are largely unknown. Some studies have already been performed describing the effect of individual vegetable components on gene expression in mouse models and human cell lines.", 10 However, often these effects were studied in relation to, for example, exposure to carcinogens. ${ }^{9}$. 11 These studies present evidence for a protective effect of single vegetable components. In order for genes to exert their effect, changes on the transcriptomic level will have to be translated to the proteome and eventually to the metabolome. Several studies have already been performed exploring the effect of consumption of single food components on protein expression in the colon and colonic cell lines. ${ }^{12.14}$ These studies focused, for example, on the regulation of specific enzymes involved in (the protection against) CRC or expression of proteins important for cell cycle regulation and apoptosis. However, not much is yet known about the effect of vegetable consumption on the total protein expression pattern.

In a previous study of our group, the effect of vegetable consumption on gene expression changes in colon mucosa from fernale mice was described (Chapter 3 ). In the current study, the effect of increased vegetable levels in the diet on the protein expression in the colon mucosa of these female mice was studied, in order to identify the proteins for which the expression levels are affected by increased vegetable consumption. Mice were fed four different diets, containing respectively $0 \%, 10 \%, 20 \%$ or $40 \%$ vegetables (mixture of cauliflower, carrots, peas and onions). Each vegetable represents a subclass of 
vegetables that are known to interact via different mechanism with the process of carcinogenesis. Cauliflower belongs to the family of cruciferous vegetables. They contain isothiocyanates and indoles, breakdown products of glucosinolates that are present in these vegetables. These compounds affect the levels of detoxification enzymes and modulate the levels of phase-I and -II metabolizing enzymes.15-17 Carrots contain antioxidants that protect the cell from free radical damage and suppress cell proliferation. ${ }^{10,}{ }^{18-20}$ Dietary fibers dilute and bind carcinogens in the digestive tract, decrease transit time of faecal bulk and inhibit cell proliferation. Peas are known to contain large amounts of fiber and are therefore added to the vegetable diets. Onion is a diallyl vegetable, containing organosulfur compounds that can modify carcinogen activation by changing biotransformating and detoxification enzyme levels. ${ }^{10,19,21,22}$

In the current study, total protein was isolated from colonic mucosal cells and the proteins were separated by 2D-gelelectrophoresis. Proteins that showed differential expression in one or more of the vegetable groups compared to control were selected for identification by MALDI-TOF mass spectrometry. Five out of six identified proteins might play a role in the protection against CRC. This may provide new insights in the biological mechanisms that possibly are related to or even directly involved in the CRC risk reduction properties of vegetables.

\section{Materials and Methods}

\section{Animals and Diets}

The number and type of animals, the development of the vegetable diets and treatment of the animals in the present study was previously described in Chapter 3 (Section Materials and Methods; Animals and diet). In short, eight-week-old female C57BL6 mice (Charles River Laboratories, France) were randomly assigned to one of four different diets, containing a $0 \%$ (referred to as control diet), $10 \%, 20 \%$ and $40 \% \mathrm{wt} /$ wt vegetables mixture respectively, for a period of two weeks (7 mice per group). The vegetables mixture used in the present mice study consisted of cauliflower ( $30 \%$ wet wt), carrots $(30 \%$ wet wt), peas $(30 \%$ wet wt), and onions ( $10 \%$ wet wt). The composition of the different diets is given in Table 1.

The animals were maintained under controlled environmental conditions (temperature $21 \pm 1{ }^{\circ} \mathrm{C}$, relative humidity $50 \% \pm 10,12$-hr light/dark cycle). Body weights of the mice were recorded weekly. The study was approved by the Institutional Committee of Animal Experimentation of the University of Maastricht. 


\section{Protein Isolation}

Protein from mouse colon mucosal cells was isolated from the residuals after the isolation of RNA using Trizolm (GIBCO Life Technologies, Breda, The Netherlands) according to the manufacturers' instructions. 'Total RNA was used for gene expression studies (Chapter 3). After precipitation of the RNA, the supernatant was collected and stored at $-80^{\circ} \mathrm{C}$ for isolation of total protein according to the manual, except that two additional wash steps of the protein pellet were performed in $0.3 \mathrm{M}$ guanidine hydrochloride in $95 \%$ ethanol and one additional wash in $95 \%$ ethanol to reduce contamination. Total protein from two to three mice was pooled obtaining in total 3 pools per diet group similar to the pools in the gene expression studies (Chapter 3). Protein concentrations were determined using the DC Protein Assay (Bio-Rad, Veenendaal, The Netherlands).

Table 1. Composition of the four diets in $\mathrm{g} / \mathrm{kg}$.

\begin{tabular}{|c|c|c|c|c|}
\hline Ingredients & Control diet ${ }^{1}$ & $\begin{array}{c}10 \% \text { Vegetable } \\
\text { diet }^{2}\end{array}$ & $\begin{array}{c}20 \% \text { Vegetable } \\
\text { diet }^{2}\end{array}$ & $\begin{array}{c}40 \% \text { Vegetable } \\
\text { diet }^{2}\end{array}$ \\
\hline Cerclose/Dextrose & 515 & 422 & 329 & 143 \\
\hline DL-Methionine & 2 & 2 & 2 & 2 \\
\hline $\mathrm{NaCl}$ & 3 & 3 & 3 & 3 \\
\hline Choline CL. $50 \%$ & 4 & 4 & 4 & 4 \\
\hline Soya oil & 50 & 50 & 50 & 50 \\
\hline $\mathrm{KCl}$ & 7 & 7 & 7 & 7 \\
\hline $\mathrm{KH}_{2} \mathrm{PO}_{4}$ & 7 & 7 & 7 & 7. \\
\hline $\mathrm{MgO}$ & 2 & 2 & 2 & 2 \\
\hline $\mathrm{CaHPO}_{4} .2 \mathrm{H}_{2} \mathrm{O}$ & 13 & 13 & 13 & 13 \\
\hline $\mathrm{CaCO}_{3}$ & 10 & 10 & 10 & 10 \\
\hline Corn Starch & 100 & 100 & 100 & 100 \\
\hline Dicacel/Cellulose & 77 & 70 & 63 & 49 \\
\hline Cascin . & 200 & 200 & 200 & 200 \\
\hline $\mathrm{MgSO}_{4} .7 \mathrm{H}_{2} \mathrm{O}$ & 4 & 4 & 4 & 4 \\
\hline $\begin{array}{l}\text { Standard vitamin } \\
\text { premix }\end{array}$ & 3 & 3 & 3 & 3 \\
\hline $\begin{array}{l}\text { Standard } \\
\text { micronutrient premix }\end{array}$ & 3 & 3 & 3 & 3 \\
\hline Vegetables mixture ${ }^{3}$ & 0 & 100 & 200 & 400 \\
\hline
\end{tabular}

1 Control diet is the basal $20 \%$ cascin reference diet.

In the vegetable diets, the basal diet was adjusted for cerelose/dextrose and dicacel/cellulose resulting in similar energy densities for all diets.

Composition (\% wet weighr): cauliflower (30\%), carrots (30\%

\section{D-Gelelectrophoresis}

$100 \mu \mathrm{g}$ of total protein of each pool was loaded on Immobiline Dry Strips (pH 3-10, $24 \mathrm{~cm}$ long) for the first dimension. Isoelectric focusing was performed on an IPGphor 
electrophoresis unit (Amersham Biosciences, Buckinghamshire, England) at $20^{\circ} \mathrm{C}$. The strips were rehydrated overnight in $350 \mu$ of $8 \mathrm{M}$ urea, $2 \%$ w/v CHAPS, $65 \mathrm{mM}$ DTT, $0.5 \% \mathrm{v} / \mathrm{v}$ IPG buffer $3-10$ at $30 \mathrm{~V}$. Isoelectric focusing was performed using the following program: $500 \mathrm{~V}$ for $1 \mathrm{~h}, 1000 \mathrm{~V}$ for $1 \mathrm{~h}, 1000-8000 \mathrm{~V}$ for $2 \mathrm{~h}$ and a final step of $8000 \mathrm{~V}$ to reach a total of $56250 \mathrm{Vh}$. After focusing, IPG strips were equilibrated for $15 \mathrm{~min}$ in 50 $\mathrm{mM}$ Tris- $\mathrm{HCl}, \mathrm{pH} 6.8,6 \mathrm{M}$ urea, $30 \% \mathrm{v} / \mathrm{v}$ glycerol, $2 \% \mathrm{w} / \mathrm{v}$ SDS, $1 \% \mathrm{w} / \mathrm{v}$ DTT and for $15 \mathrm{~min}$ in $50 \mathrm{mM}$ Tris- $\mathrm{HCl}, \mathrm{pH} 6.8,6 \mathrm{M}$ urea, $30 \% \mathrm{v} / \mathrm{v}$ glycerol, $2 \% \mathrm{w} / \mathrm{v} \mathrm{SDS}, 2.5 \% \mathrm{w} / \mathrm{v}$ iodoacetamide, and were placed onto a slab gel and sealed with a $0.5 \% \mathrm{w} / \mathrm{v}$ agarose solution in Laemmli buffer with a trace of bromophenol blue. The second-dimension run was carried out on $12.5 \%$ SDS-PAGE gels. Electrophoresis was conducted at a constant voltage of $200 \mathrm{~V}$ for $5 \mathrm{~h}$ in a $24 \mathrm{~cm}$ Dodeca Cell (Bio-Rad) (Rabilloud et al., 1997; Gorg et al., 2000). In-gel proteins were silver-stained according to Humphery-Smith et al. (2000). Gel images were obtained with a GS-800 calibrated densitometer (Bio-Rad).

\section{Spot Selection}

Protein expression patterns were analyzed using the PDQuest 7.1.1 program (Bio-Rad). The average spot density from the three replicate gels of each diet group was calculated and the protein spots were analyzed for differential protein expression upon consumption of vegetables by comparison of these average values to those of the control diet. Proteins that showed at least two times enhanced or decreased expression were selected for identification.

\section{In-gel Protein Digestion}

Protein spots were excised from the gel and processed on a MassPREP digestion robot (Waters, Manchester, UK) according to the following protocol: for silver stained spots $0.5 \% \mathrm{H}_{2} \mathrm{O}_{2}$ was used for destaining. Cysteines were reduced with $10 \mathrm{mM}$ dithiothreitol in $100 \mathrm{mM}$ ammonium bicarbonate for 30 minutes followed by alkylation with $55 \mathrm{mM}$ iodoacetamide in $100 \mathrm{mM}$ ammonium bicarbonate for 20 minutes at room temperature (RT). Spots where washed with $100 \mathrm{mM}$ ammonium bicarbonate to remove excess reagents and dehydrated with $100 \%$ acetonitrile. Trypsin $(6 \mathrm{ng} / \mu \mathrm{l})$ in $50 \mathrm{mM}$ ammonium bicarbonate was added to the gel plug and incubated at $37{ }^{\circ} \mathrm{C}$ for 5 hours. The peptides were extracted with $1 \%$ formic acid / $2 \%$ acetonitrile.

\section{Mass Spectrometry}

For MALDI-TOF mass spectrometry $1.5 \mu \mathrm{l}$ of each peptide mixture and $0.5 \mu \mathrm{l}$ matrix solution (10 $\mathrm{mg} / \mathrm{ml} \alpha$-cyano-4-hydroxycinnamic acid in $50 \%$ acetonitrile $/ 0.1 \% \mathrm{TFA}$ ) was spotted automatically onto a 96 well-format target plate. The spots were allowed to air dry for homogenous crystallization. Spectra were obtained using a M@ldi-L.R mass spectrometer (Waters, Manchester, UK). The instrument was operated in positive 
reflectron mode. Acquisition mass range was 900-3000 Da. The instrument was calibrated on 6-8 reference masses from a tryptic digest of alcohol dehydrogenase. Next, a nearpoint lockmass correction for each spot was performed using ACTH 1-39 $(\mathrm{MH}+2465.199)$ to achieve maximum mass accuracy. Typically 100 shots were combined and background subtracted. A peptide mass list was generated for subsequent database search. ${ }^{23}$

\section{Database Seamb of M/ALDI-TOF Peptide Spectra}

The peptide mass list was searched with Mascot (http://www.matrixscience.com) against the Swiss-Prot database (http://expasy.ch/sprot) for protein identification. The search parameters were as follows: one mis-cleavage was tolerated; carbamidomethylation was set as a fixed modification; and oxidation of methionine as an optional modification. The peptide mass tolerance was set to $100 \mathrm{ppm}$. No restrictions were made on the protein molecular weight and the isoelectric point. A protein was regarded identified when the search was found to be statistically significant $(\mathrm{p}<0.05)$ in Mascot.

\section{Immunoblotting}

Total cellular proteins were separated by SDS-PAGE: (12\% acrylamide) followed by immunoblotting. $10 \mu \mathrm{g}$ of total protein was dissolved in $1 \%$ SDS and $30 \mathrm{mM}$ DTT, 15 $\mathrm{mM}$ Tris- $\mathrm{HCl}$ buffer ( $\mathrm{pH} 6.8$ ), $10 \%$ glycerol and $0.006 \%$ bromophenol blue (final concentrations). SDS-polyacrylamide gelelectrophoresis was performed in $12 \%(\mathrm{w} / \mathrm{v})$ slab gels using the (mini) Proteanll equipment (Bio-Rad). After separation, the proteins were electrophoretically transferred to nitrocellulose paper (Schleicher \& Schuell, Dassel, Germany) in blotting buffer (25 mM Tris, $200 \mathrm{mM}$ glycine, $20 \%$ methanol) using the (mini)Trans-blot (Bio-Rad). Subsequently free protein binding sites on the nitrocellulose filter were blocked with $5 \%$ BSA (w/v) and $0.1 \%$ Tween- $20(v / v)$ in PBS for 1 hour at RT under gently agitation. The blots were incubated with the first antibody in PBS, $0.05 \%$ Tween-20 for 1 hour at RT or o/n at $4^{\circ} \mathrm{C}$. Antibodies used were: anti-GAPDH 1:10.000 (Campro, Veenendaal, The Netherlands), anti-HMG1 1:5.000 (United States Biological, Swampscott, USA) and anti-CAH1 1:20.000 (United States Biological). After rinsing in excess PBS, $0.05 \%$ Tween-20, detection was accomplished using an appropriate chemoluminescent secondary antibody (Abcam, Cambridge, UK); rabbit-anti-mouse IgG peroxidase-conjugated 1:10.000 in PBS, $0.05 \%$ Tween-20 for GAPDH or 1:5.000 for HMG-1 and rabbit-anti-goat IgG peroxidase-conjugated 1:60.000 in PBS, 0.05\% Tween-20 for $\mathrm{CAH}-1$, for 1 hour at RT. After rinsing in excess PBS, $0.05 \%$ Tween- 20 the blots were incubated for $5 \mathrm{~min}$ at RT with ECL plus Western blotting detection reagent (Amersham Biosciences) according to the manufacturer's instructions. The signal was recorded using a LAS 3000 camera in combination with the Las-3000 pro program (Fuji, Tilburg. The Netherlands). Molecular weight values were estimated using standard molecular weight 
markers. Protein band densities were calculated and corrected for background using Aïda version 3.5 (Raytest Benelux B.V. Tilburg, The Netherlands).

\section{Statistical Analysis}

Statistical analysis of protein expression was performed using the Student's t-test carried out with SPSS software version 6.1 for Macintosh. A p-value $<0.05$ was considered as statistical significant.

\section{Results}

\section{D-Gelelectnoporesis and MAIDI-TOF Mass Spectnometry}

Mice received one of four different diets, containing a $0 \%, 10 \%, 20 \%$ or $40 \%$ wt $/$ wt vegetables mixture respectively, for a period of two weeks. Differential protein expression in colonic mucosal cells was analysed by means of $2 \mathrm{D}$-gelelectrophoresis. Single gels were prepared from pooled total cellular protein obtained from two to three animals. In total, three gels were run for each diet. Spot densities were calculated as a measure for protein expression and the expression in the diet groups was compared to that of mice receiving the control diet $(0 \%$ vegetables).

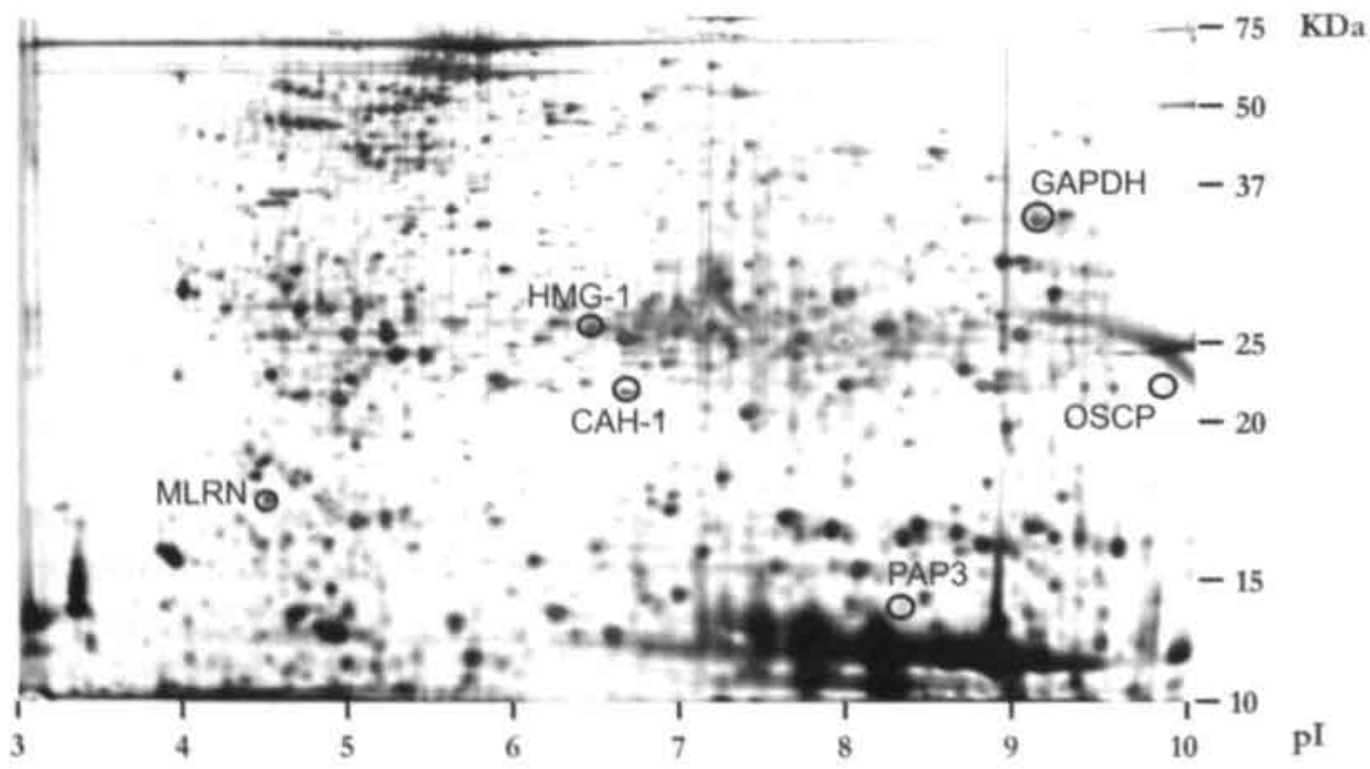

Figure 1. Silver stained 2D-gel of proteins isolated from mouse colonic mucosal cells. The spots for which the protein identity was revealed are indicated on the gel (see Table 2 for protein funcrion). 
Spots that showed at least two times increased or decreased protein expression compared to control were selected for protein identification using MALDI-TOF mass spectrometry. From the 30 excised proteins, 6 could be identified. Figure 1 shows an example of a $2 \mathrm{D}$ gel together with the identified proteins. An example of a peptide spectrum of one of the identified proteins is depicted in Figure 2. These spectrometric data were used to search the Swiss-Prot protein database in order to reveal the identity of the proteins.

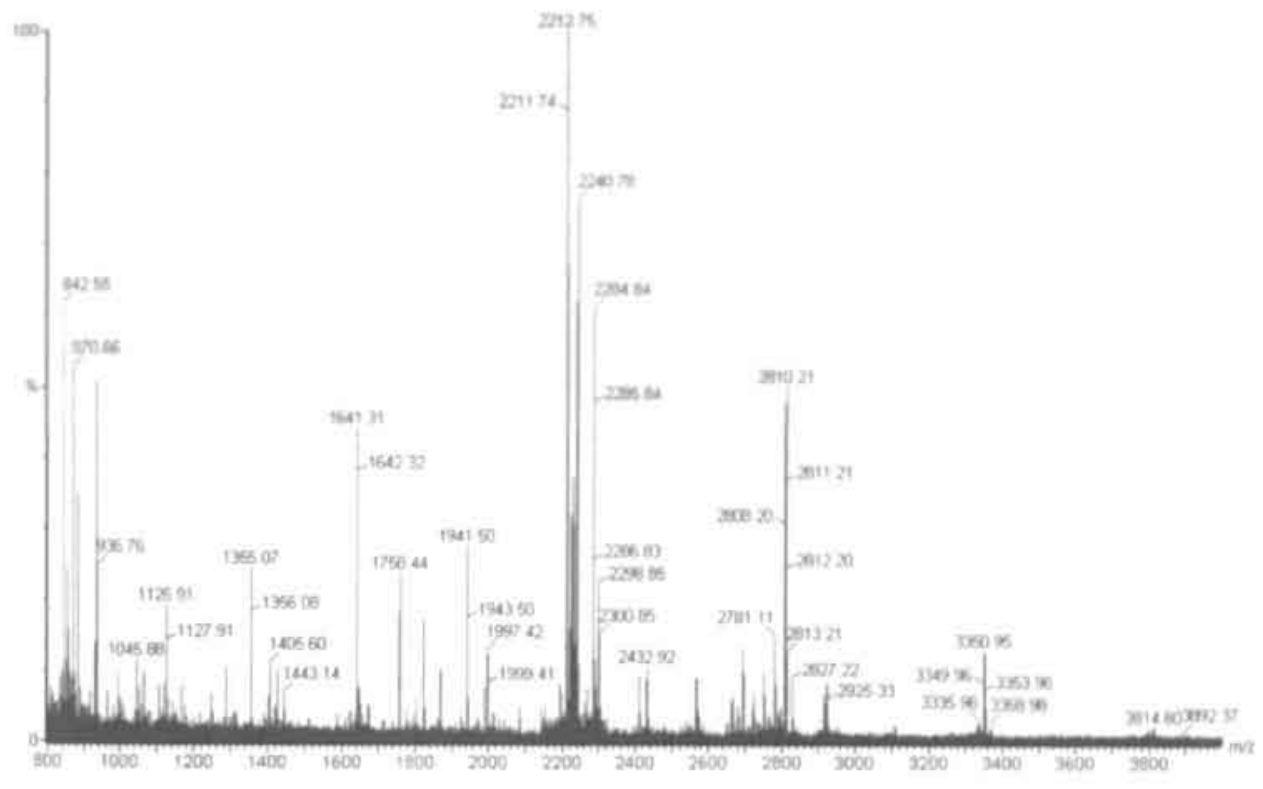

Figure 2. Tryptic MALDI-TOF spectrum of CAH-1.

The expression patterns of the identified proteins are shown in Figure 3 and their properties have been summarized in Table 2. Spot 1222 was identified as the smooth muscle isoform of myosin regulatory light chain 2 (MLRN). ML.RN expression was very similar to control in both the $10 \%$ and $20 \%$ vegetable groups, but showed an increased expression for the $40 \%$ vegetable group. Spot 5316 represents carbonic anhydrase I (CAH1) and was upregulated in all vegetable groups, although this upregulation was statistically significant only for the $20 \%$ vegetable group. The peptide fragment pattern obtained from protein spot 5419 after trypsin digestion was found to match the high-mobility group protein 1 (HMG-1).The expression of this protein was decreased upon vegetable consumption, but this was not statistically significant. Spot 7076 was identified as pancreatitis-associated precursor protein 3 (PAP3). This protein was absent in colon mucosa from control mice, displayed only low expression in the $10 \%$ and $20 \%$ vegetable groups, but was significant increased in the $40 \%$ vegetable group. Spot 8641 was found to 
represent glyceraldehyde-3-phosphate dehydrogenase (GAPDH). All vegetable groups showed reduced expression of GAPDH compared to control mice. The peptide fragment mass spectrum obtained from protein spot 9241 was found to match the mitochondrial ATP synthase oligomycin sensitivity conferral precursor protein (OSCP), and its protein expression was reduced in all vegetable groups compared to control.

Table 2. Protein spots isolated from 2D-gels identified by MALDI-TOF mass spectrometry in combination with searches in the Swiss-Prot protein database.'

\begin{tabular}{|c|c|c|c|c|}
\hline Spot \# & $\mathbf{A C} \#$ & $\begin{array}{l}\text { Swiss-prot } \\
\text { abbreviation } 1\end{array}$ & Abbreviation 1 & Protein name ${ }^{1}$ \\
\hline 1222 & Q9CQ19 & MI.RN_MOUSE & MILRN & $\begin{array}{l}\text { myosin regulatory light chain } 2 \text {, smooth } \\
\text { muscle isoform }\end{array}$ \\
\hline 5316 & P13634 & CAH1_MOUSE & $\mathrm{CAH}-1$ & carbonic anhydrase 1 \\
\hline 5419 & P07155 & HMG1_MOUSE & HMG-1 & high mobility group protein 1 \\
\hline 7076 & 009049 & PAP3_MOUSE & PAP3 & $\begin{array}{l}\text { pancreatitis-associated protein } 3 \\
\text { (precursor) }\end{array}$ \\
\hline 8641 & P16858 & G3P_MOUSE & GAPDH & $\begin{array}{l}\text { glyceraldehyde 3-phosphate dehydrogenase } \\
\text { ATP synthase oligomycin sensitivity }\end{array}$ \\
\hline 9241 & Q9DB20 & ATPO_MOUSE & OSCP & $\begin{array}{l}\text { conferral protein, mitochondrial } \\
\text { (precursor) }\end{array}$ \\
\hline
\end{tabular}

'(hrtp://expasy.ch/sprot)

\section{Immunoblotting}

Immunoblotting was performed with three out of six identified proteins, as for these antibodies were commercially available. Figure $4 \mathrm{~A}$ shows the protein expression patterns of respectively HMG-1, GAPDH and $\mathrm{CAH}-1$, and Figure $4 \mathrm{~B}$ shows the relative protein expression of the identified proteins in the vegetable groups compared to control. The 25 $\mathrm{kDa}$ band for HMG-1 showed a dose dependent upregulation compared to control, which was found to be significant for the $10 \%$ and $40 \%$ vegetable groups, but not the $20 \%$ vegetable group. This is in contradiction to its 2D-expression pattern where HMG-1 showed no clear change in protein expression. GAPDH showed a band at $36 \mathrm{kDa}$. The 2D-protein expression pattern indicated decreased protein expression in all vegetable groups. The immunoblot results for GADPH showed a highly variable expression pattern suggesting that there is no dose dependent effect of vegetable consumption on GADPH expression. Possibly, the strong variation in the expression pattern masks a possible change in protein expression upon consumption of the vegetable-enriched diets. $\mathrm{CAH}-1$ showed a single band at $28 \mathrm{kDa}$ (Figure $4 \mathrm{~A}$ ) and was significantly upregulated in all vegetable groups compared to control (Figure 4B) as this was also observed in the 2D-gelectrophoresis expression pattern. 
A

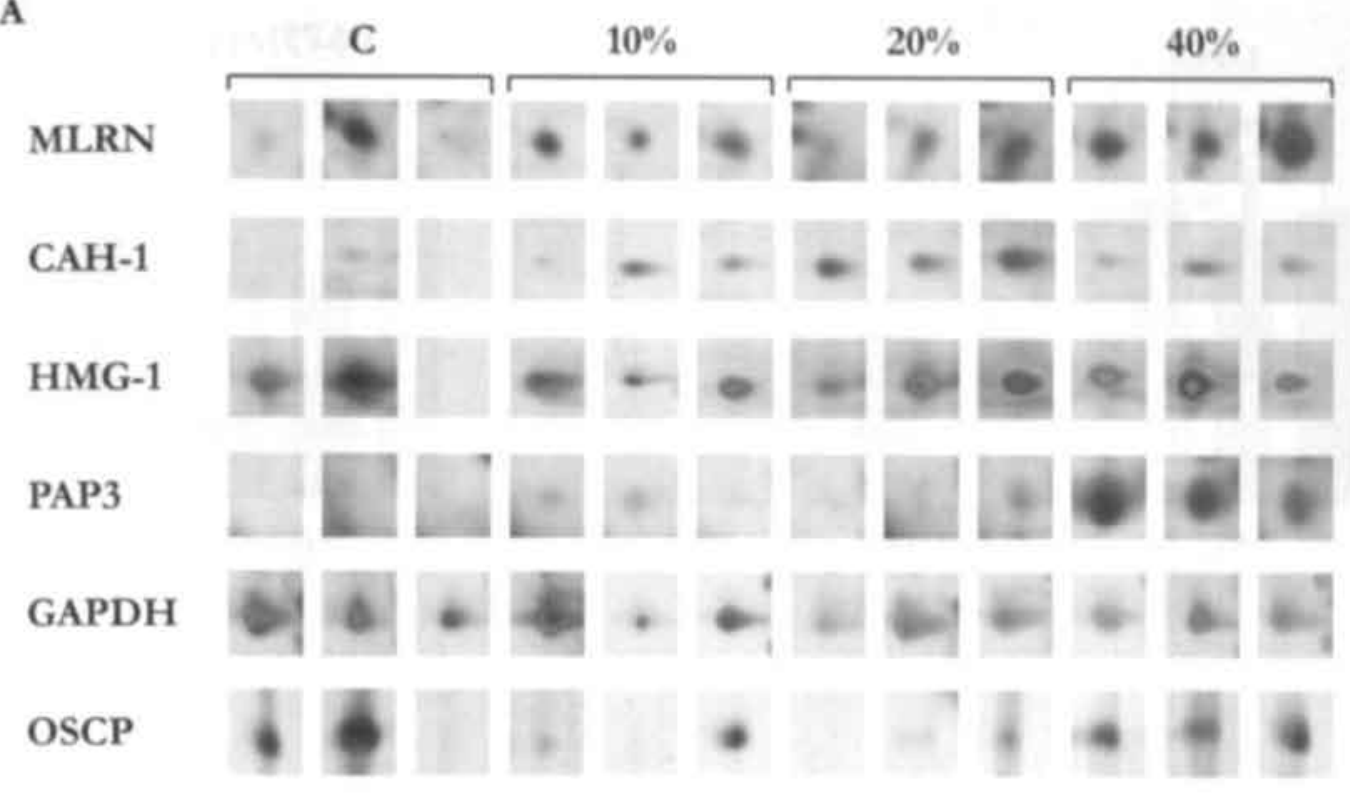

B
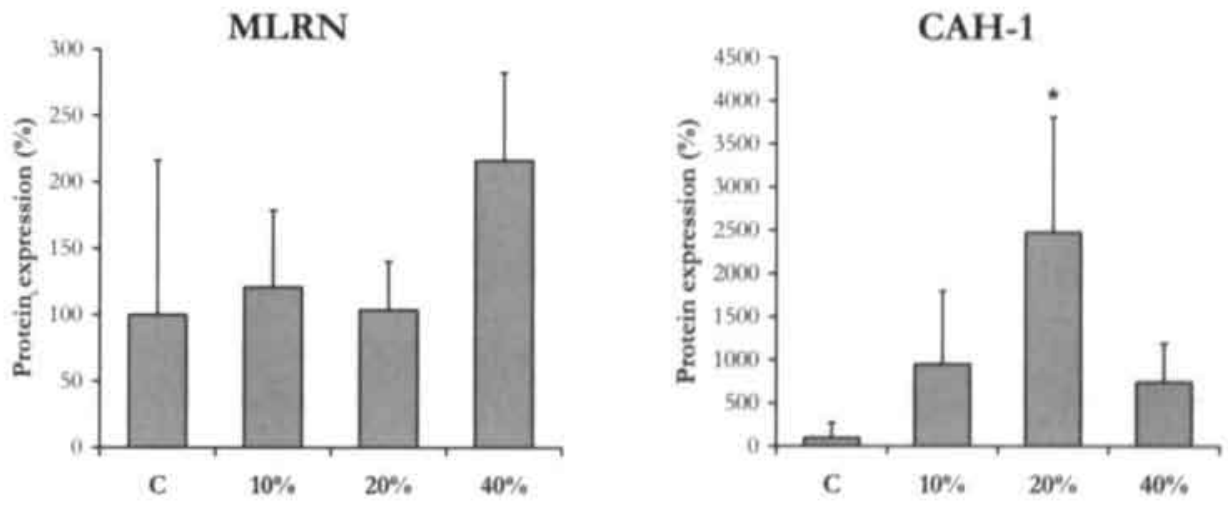

Figure 3. (A) Expression partern of the identified spots from 2D-gels. (B) Relative protein expression of the identified spots compared to control Average protein level for controls was set at $10 \%^{\circ},{ }^{*} p<0.05$. MI.RN: smooth muscle isoform of myostn regulatory light chain 2; CAH-1: carbonic anhydrase 1; HMG.1: high-mobility group protein 1; PAP3: pancreatitisassociated precursot protein 3; GAPDH: glyceraldehyde-3-phosphate dehydrogenase; OSCP: mitochondral ATP synthase oligomycin sensitivity conferral precursor protein. X-axis: $\mathrm{C}$ : control dict, $10 \% \% 10 \%$-diet, $20 \% \% .20 \%$-diet, $40 \% \% .40 \%$-diet. The third spot of the control group for HMG-1 was regarded as missing value. 

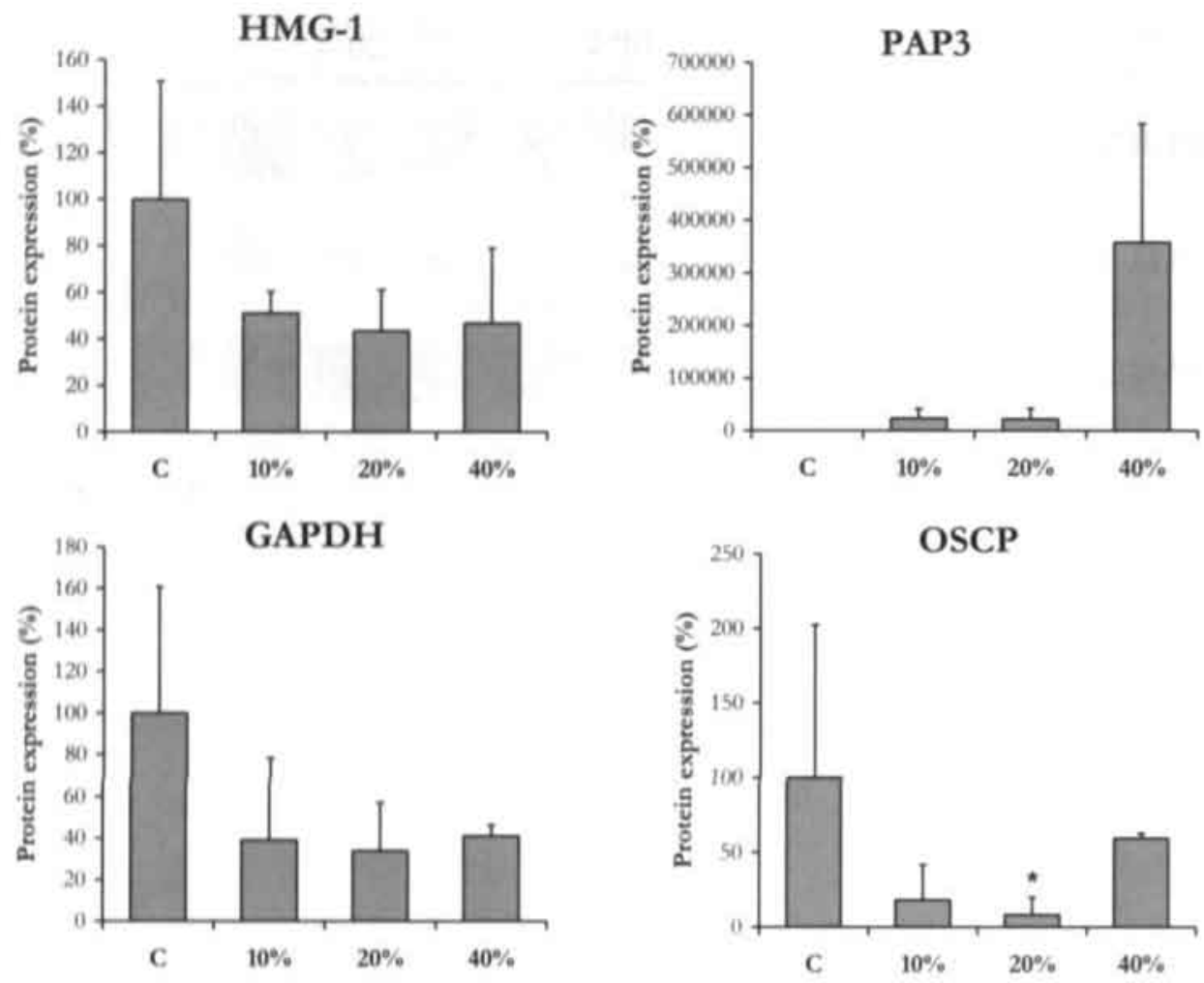

Figure 3. (Continued)

A

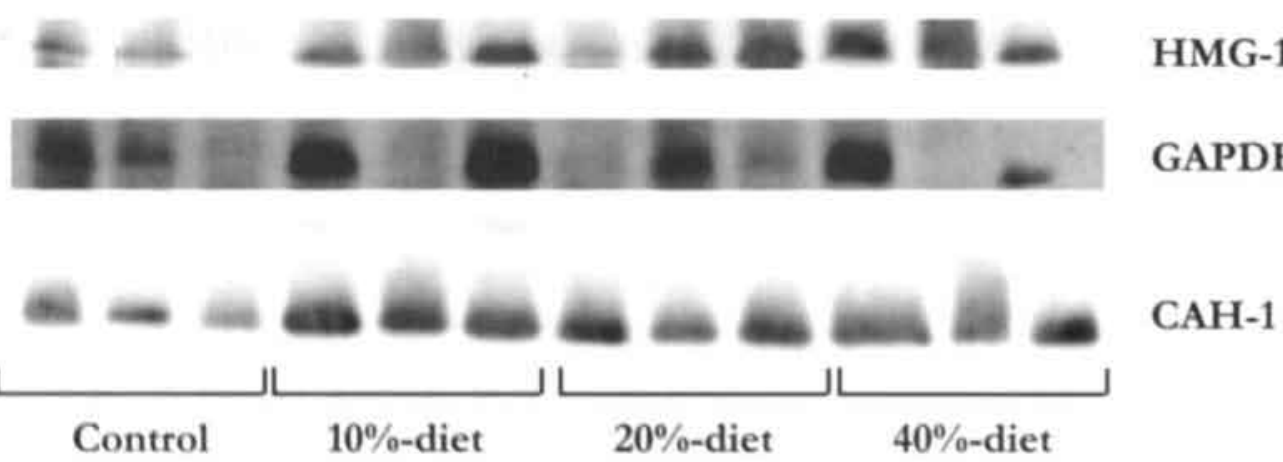

Figure 4. Immunoblot analysis of total colonic mucosal protein. (A) Blots were screened wrth antibodies: A1: HMG-1: highmobility group protein 1, A2: GAPDH: glyceraldehyde-3-phosphate dehydrogenase; and A 3: CAH-1: carbontc anhydrase I. The lanes were loaded with $10 \mu \mathrm{g}$ of total protein. (B) Protein expression relative to control of HMG-1, G.APDH and CAH-

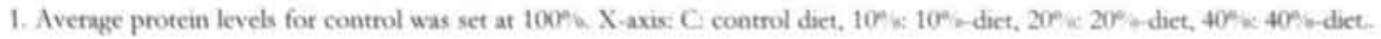
- $\mathrm{p}<0.05$. 

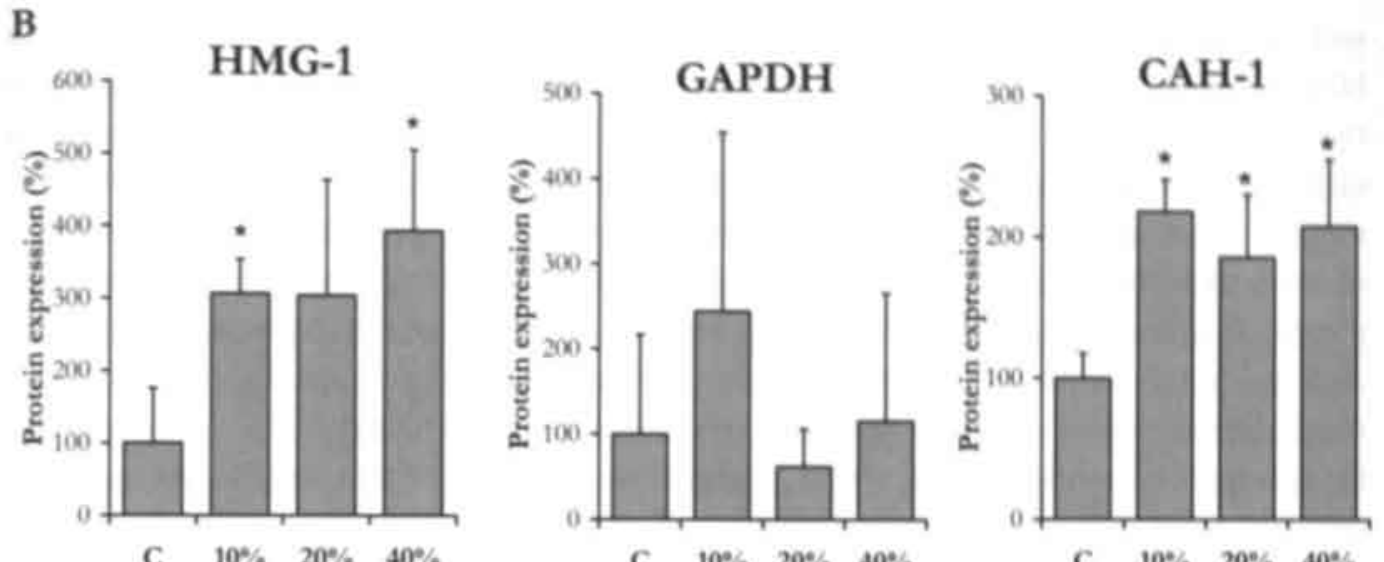

Figure 4. (Continued)

\section{Discussion}

Diet plays an important role in CRC. Meat consumption and high fat intake, for example, are known risk factors. On the other hand, vegetable consumption is thought to protect against CRC. So far, not much is known about the changes at the genome and proteome level that may be related to or involved in this protective mechanism. To study the effect of consumption of a vegetable rich diet on protein expression, we analyzed the expression levels of many proteins in colonic mucosal cells from mice following a two weeks diet with different vegetable content. Total cellular protein was isolated and the proteins were separated by means of 2D-gelelectrophoresis and identified by MALDI-TOF mass spectrometry. We were able to identify six proteins that showed a change in expression level, namely HMG-1, GAPDH, CAH-1, PAP3, OSCP and MLRN. HMG-1, GAPDH and $\mathrm{CAH}-1$ were further analysed by immunoblotting. All six proteins will be discussed in detail below.

The HMG-1 protein is both a nuclear factor and a secreted protein. In the nucleus HMG-1 binds to DNA and interacts with several transcription factors to regulate transcription ${ }^{24}$, outside the cell it is thought to be a mediator of differentiation. ${ }^{25}, 26$ Analysis of HMG-1 protein expression using 2D-gelelectrophoresis showed a decrease in protein expression for all vegetable groups compared to control, although this was not statistically significant. This is in contradiction with the expression pattern observed from the immunoblotting experiments, where we found that HMG-1 expression was significantly upregulated upon increased vegetable consumption, which was dosedependent. This difference in protein expression pattern between 2D-gelelectrophoresis 
and immunoblotting might be explained by posttranslational modification of the protein. HMG-1 is thought to be regulated by phosphorylation. ${ }^{27}$ Hypophosphorylation enhances transport to the nucleus and subsequent binding to DNA.27 The same protein but with different phosphorylation status will appear on different locations on the gel. For this moment it is unknown whether we detected the phosphorylated or hypophosphorylated isoform of HMG-1. Since immunoblotting reflects total protein expression, its expression pattern observed may differ from that observed upon 2D-gelelectrophoresis. The cells analyzed in this study are healthy colonic mucosal cells, containing dividing stem cells and many differentiating crypt and epithelial cells. Since phosphorylated HMG-1 is important in cellular differentiation, increased expression of this isoform might enhance cell differentiation, which suppresses aberrant cell growth of poorly differentiated cells like many (pre-)cancerous cells, thereby reducing the risk for CRC. However, for the moment we do not know which isoform is increased upon vegetable consumption.

GAPDH is a cytoplasmic protein that has long been considered as a classical glycolytic enzyme, playing an important role in energy production. ${ }^{28}$ The active glycolytic enzyme consists of a tetramer of four identical subunits. However, mammalian GAPDH displays a wide functional diversity including membrane transport and fusion, microtubuli assembly, nuclear RNA export, protein phosphotransferase and kinase reactions, translational control of gene expression, DNA replication and repair and apoptosis. ${ }^{20}$ Our 2D-gelelctrophoresis experiments indicated a substantially decreased protein expression in all vegetable groups compared to control, although this decrease was not statistically significant. The immunoblotting results on the other hand showed a large variation between the various sub-pool samples due to which no clear differences in protein expression between the four diets can be observed. So far, we can not explain this variation. For the six proteins that were identified by MALDI-TOF mass spectrometry, GAPDH was the only protein of which the gene was present in the pool of genes that were analysed in the microarray study (Chapter 3). In this study, an increased GAPDH gene expression in the $40 \%$ vegetable group was found, but the expression in the $10 \%$ and $20 \%$ vegetable groups did not change. The outcome of these results was confirmed by RTPCR. This pattern differs from that which was found in the present study in the 2Dgelectrophoresis. Although Piechaczyk et al. (1984) observed similar changes in expression for GAPDH protein and mRNA levels in several rat organs under normal conditions ${ }^{30}$, no such relation was found in our experiments. The difference between the mRNA levels and protein levels may be due to different subforms of GAPDH. To exert the wide variety of GAPDH functions, the different subforms of the protein need to be distinguished by the cell. This might be regulated by alternate splicing or posttranslational modification and give protein products that differ in isoelectric point $(\mathrm{pl})$ and/or molecular weight. ${ }^{20}, 31$ This might also explain the difference in protein expression as observed from the 2D. gelelectrophoresis and the immunoblotting experiments. Considering the role of GAPDH 
in DNA replication, DNA repair and apoptosis ${ }^{29}$, GAPDH might be an interesting candidate for protection against CRC. However, the evidence so far is too premature to draw definitive conclusions.

CAH-1 is a member of a family of isoenzymes that catalyze the reversible hydration of carbon dioxide and participate in various biological processes like electrolyte transport and maintenance of $\mathrm{pH}$. The isoenzymes differ in kinetic properties, tissue distribution and subcellular localization. $\mathrm{CAH}-1$ is a cytoplasmic isoenzyme that is highly expressed in large intestinal mucosa ${ }^{32}$ and takes care of the maintenance of the intracolonic $\mathrm{pH}$. In colorectal tumours CAH-1 is significantly less expressed than in normal mucosa." Reduced expression of $\mathrm{CAH}-1$ might lead to cellular acidic $\mathrm{pH}$, thereby promoting cellular motility, and contribute to tumour growth and metastasis. ${ }^{34}$ Our results from the 2D. experiments already indicated an increase in protein expression upon vegetable consumption, although this was only significant for the $20 \%$ vegetable group. The immunoblot experiments showed that $\mathrm{CAH}-1$ protein expression is significantly upregulated in all vegetable groups compared to control. We therefore suggest that consumption of a vegetable rich diet contributes to maintenance of the intracolonic and cellular $\mathrm{pH}$, which thereby might protect against CRC development.

PAP3 belongs to the regenerating gene (Reg) family that constitutes the calcium dependent lectin (C-type lectin) gene superfamily. ${ }^{35}$ The Reg family represents a group of small secretory proteins that can function as acute phase reactants, lectins, anti-apoptotic factors and growth factors. The members of the Reg family can be grouped into three subclasses: type 1, II and III. ${ }^{35}$ There are at least three distinct type III Reg genes in the mouse genome: Reg III alpha (RegIII $\alpha)$, beta $(\operatorname{RegIII} \beta)$ and gamma (RegIII $\gamma$, i.e. PAP3). ${ }^{\text {* }}$ The subclasses share considerable amino acid homologies including a conserved trypsin cleavage site. It is thought that the secreted forms represent the precursors and the cleaved forms the activated molecules. ${ }^{37}$ Mouse type III Reg genes are expressed strongly in intestinal tracts and PAP3 in particular is expressed strongly in small intestine, moderately in colon and weakly in pancreas. ${ }^{35}$, 36 The type 111 genes are thought to be involved in the growth and regeneration of cells and tissues. In our 2D-gelectrophoresis experiments we identified the PAP3 precursor protein. It was upregulated in the $40 \%$ vegetable groups, while hardly any effect was observed in the $10 \%$ and $20 \%$ vegetable group. Our results suggest that only a high intake of vegetables $(40 \%)$ stimulates the expression of PAP3 precursor protein. Considering the role of PAP3 in growth and regeneration of cells and tissues, we suggest that activation of the precursor might lead to increased amounts of active PAP3 protein, stimulating the proliferation of healthy cells and/or regeneration of damaged tissue and thus contribute to healthy colon tissue. However, to allow regeneration, it might be necessary to switch off apoptosis, thereby increasing the chance of survival of cells exhibiting genetic mutations in growth promoting or metastasis inducing genes. ${ }^{38}$ Thus, upregulation of PAP3 precursor protein and thereby possibly of 
PAP3, might contribute to proliferation and differentiation of healthy colon tissue although it also involves a risk for CRC. Upregulation of the precursor protein of PAP3, however, may not necessarily lead to a change in active protein levels. Additional experiments are therefore needed to unravel the regulation of PAP3 protein expression.

ATP production is one of the major chemical reactions in living organisms. ATP synthase is a key enzyme in cellular energy production. During ATP synthesis it uses a proton gradient and the associated membrane potential to synthesize ATP. The ATP synthases are rotary motor complexes that are composed of two discrete sectors $\left(\mathrm{F}_{1}\right.$ and $\left.\mathrm{F}_{0}\right)$ that are considered to be two separate rotary motors working cooperatively. Oligomycin sensitivity-conferring protein (OSCP) is a component of the $\mathrm{F}_{0}$ sector and is considered to serve as a link between the $F_{1}$ and $F_{0}$ sectors, ${ }^{39}$ OSCP also has been implicated in the binding of $\mathrm{F}_{1}$ to the membrane.40, 41 In our 2D-gelelctrophoresis experiments we observed a downregulation of the OSCP precursor protein upon increased vegetable consumption that was only statistically significant for the $20 \%$ vegetable group. Considering the fact that OSCP precursor protein and the active OSCP protein are very likely to be located at different locations on the 2D-gel, reduced expression of OSCP precursor protein might indicate that the amount of precursor is decreased in favour of active, processed OSCP. The processed OSCP might then be used for assembly of ATP synthase and contribute to ATP production. At this moment there are no indications that OSCP might play a role in CRC.

Myosin is a major component of the contractile elements of smooth muscle and is composed of two identical heavy chains $(200 \mathrm{kDa})$ and two sets of light chains of 20 and $17 \mathrm{kDa}^{42}$ The $20 \mathrm{kDa}$ chain, called regulatory light chain (MLRN), plays a central role in the regulation of smooth muscle contraction. ${ }^{43}$. 44 In our 2D-gelelectrophoresis experiments we found an increase, although not significant, of MLRN protein expression with increasing vegetable intake, most evident for the $40 \%$ vegetable group. This might indicate that vegetable consumption increases smooth muscle activity. Vegetables contain high amounts of dietary fibers and are known to stimulate bowel movements, decreasing the passage time through the gut. $45,{ }^{4}$ Mutagenic agents might be present in faeces and decreased passage time reduces the risk of interaction with the colon tissue and protects against genetic damage. Increased MLRN protein expression might contribute to enhanced bowel movement and as a consequence reduce the passage time of mutagenic agents, lowering the risk for genetic damage and CRC.

In the current study, several proteins were identified that displayed differential expression levels in colon mucosa of mice following consumption of diets with different vegetable contents. These proteins play a role in processes like growth, differentiation and apoptosis. Disturbance of these processes plays an important role in carcinogenesis. We therefore conclude that altered protein levels following increased vegetable intake, support the protective effects of vegetables against CRC. 


\section{References}

1. Greenwald P. Colon cancer overvicw. Cancer 1992;70:1206-15.

2. Plazz EA, Willetr WC, Coldita GA, Rimm EB, Spicgelman D, Giovannucei E. Proportion of colon cancer risk that might be preventable in a cohort of middle-aged US men. Cancer Causes Control 2000,11:579-88.

3. World Cancer Rescarch Fund. Food, Nutrition and the Prevention of Cancer: a global perspective. Washington: BANTA Book group, 1997.

4. Slartery MI, Curtin KP, Edwards SI, Schaffer DM. Plant foods, fiber, and rectal cancer, Am J Clin Nutr 2004;79:274-81.

5. Riboli E, Norat T. Epidemiologic evidence of the protective effect of fruit and vegetables on cancer risk. Am J Clin Nutr 2003;78:559S-569S.

6. Finley JW. Reduction of cancer risk by consumption of selenium-enriched plants: enrichment of broccoli with selenium increases the anticarcinogenic properties of broccoli. I Med Food 2003;6:19. 26.

7. Kallay E, Adlerereutz H, Farhan H, Lechner D, Bajna E, Gerdenitsch W, Campbell M, Cross HS. Phytoestrogens Regulate Vitamin D Metabolism in the Mouse Colon: Relevance for Colon Tumor Prevention and Therapy. J Nutr 2002;132:34908-3493S.

8. Mahmoud NN, Carothens AM, Grunberget D, Blinski RT, Churchill MR, Martucci C. Newmark HI. Bertagnolli MM. Plant phenolics decrease intestinal tumon in an animal model of familial adenomatous polyposis. Carcinogenesis 2000;21:921-7.

9. Symolon H, Schmelz EM, Dillehay DI, Merrill AH, Jr. Dietary soy sphingolipids suppress tumorigenesis and gene expression in 1,2-dimethylhydrazine-treated CF1 mice and ApcMin/+ mice. J Nutr 2004;134:1157-61.

10. Bertram JS, Bortkiewicz H. Dietary carotenoids inhibit neoplastic transformation and modulate gene expression in mouse and human cells. Am J Clin Nutr 1995;62:1327S-1336S.

11. Talalay P, Fahey JW. Phytochernicals from cruciferous plants protect against cancer by modulating carcinogen metabolism. J Nutr 2001;131:3027S-33S.

12. Sengupta A, Ghosh S, Bhatracharjec S, Das S. Indian food ingredients and cancer prevention - an experimental evaluation of anticarcinogenic effects of garlic in rat colon. Asian Pac J Cancer Prev 2004:5:126-32.

13. Parnaud G, Li P, Cassar G, Rouimi P, Tulliez J, Combaret 1., Gamet-Payrastre L. Mechanism of Sulforaphane-Induced Cell Cycle Arrest and Apoptosis in Human Colon Cancer Cells. Nutr Cancer 2004;48:198-206.

14. Chung JG, Lu HF, Yeh CC, Cheng KC, Lin SS, Iee JH. Inhibition of N-acetyltransferase activity and gene expression in human colon cancer cell lines by diallyl sulfide. Food Chem Toxicol 2004;42:195-202.

15. Katchamart S, Williams DE. Indole-3-carbinol modulation of hepatic monooxygenases CYP1A1, CYP1A2 and FMO1 in guinea pig, mouse and rabbit. Comp Biochem Physiol C. Toxicol Pharmacol 2001:129:377-84.

16. Larsen-Su S, Williams DE. Dietary indole-3-carbinol inhibits FMO activity and the expression of flavin-containing monooxygenase form 1 in rat liver and intestine. Drug Metab Dispes 1996:24:92731.

17. Smith T], Yang CS. Effect of organosulfur compounds from garlic and cruciferous vegetables on drug metabolism enzymes. Drug Metabol Drug Interact 2000;17:23-49. 
18. Steinmetz KA, Potter JD. Vegetables, fruit, and cancer prevention: a review. J Am Diet Assoc 1996:96:1027-39.

19. Steinmetz KA, Potter JD. Vegetables, fruit, and cancer. II. Mechanisms, Cancer Causes Control $1991 ; 2: 427-42$.

20. Bertram JS, Carotenoids and gene regulation. Nutr Rev 1999;57:182-91.

21. Guyonnet D, Siess MH, Le Bon AM, Suschetet M. Modulation of phase II enzymes by organosulfur compounds from allium vegetables in rat tissues. Toxicol Appl Pharmacol 1999:154:50-8.

22. Wargovich MJ. Experimental evidence for cancer preventive elements in foods. Cancer Lett 1997;114:11-7.

23. Bouwman F, Renes J, Mariman E. A combination of protein profiling and isotopomer analysis using matrix-assisted laser desorption/ionization-time of flight mass spectrometry reveals an active metabolism of the extracellular matrix of 3T3- $\mathrm{LI}$ adipocytes. Proteomics, in press.

24. Bianchi ME, Beltrame M. Upwardly mobile proteins. Workshop: the role of HMG proteins in chromatin structure, gene expression and neoplasia. EMBO Rep 2000:1:109-14.

25. Passalacqua M, Patrone M, Picotti GB, Del Rio M, Sparatore B, Melloni E, Pontremoli S. Stimulated astrocytes release high-mobility group 1 protein, an inducer of $\mathrm{LAN}-5$ neuroblastoma cell differentiation. Neuroscience 1998;82:1021-8.

26. Sparatore B, Passalacqua M, Patrone M, Melloni E, Pontremoli S. Extracellular high-mobility group 1 protein is essential for murine erythroleukaemia cell differentiation. Biochem J 1996:320 ( $\mathrm{Pt}$ 1):253-6.

27. Poser I, Golob M, Buettner R, Bosserhoff AK. Upregulation of HMG; leads to melanoma inhibitory activity expression in malignant melanoma cells and contributes to their malignancy phenotype. Mol Cell Biol 2003;23:2991-8.

28. Harris JI, Waters. M. Glyceraldehyde-3-phosphate dehydrogenase. In: Boyer PD, ed. The enzymes. New York: Academic Press, 1976:1-49.

29. Sirover MA. New insights into an old protein: the functional diversity of mammalian glyceraldehyde3 phosphate dehydrogenasc. Biochim Biophys Acta 1999;1432:159-84.

30. Piechaczyk M, Blanchard JM, Marty L, Dani C, Panabieres F, El Sabouty S, Fort P, Jeanteur P. Posttranscriptional regulation of glyceraldehyde-3-phosphate-dehydrogenase gene expression in rat tissues. Nucleic Acids Res 1984;12:6951-63.

31. Mezquita J, Pau M, Mezquita C. Several novel transcripts of glyceraldehyde-3-phosphate dehydrogenase expressed in adult chicken testis. J Cell Biochem 1998;71:127-39.

32. Lonnerholm G, Sclking O, Wistrand PJ. Amount and distribution of carbonic anhydrases $C A I$ and CA II in the pastrointestinal tract. Gastroenterology 1985;88:1151-61.

33. Kivela NJ, Saarrio J, Karttunen TJ, Kivela J. Parkkila AK, Pastorekova S, Pastorek J. Waheed A, Sly WS, Parkkala TS, Raianiemi H. Differential expression of cytoplasmic carbonic anhydrases, CA I and II, and membrane-associated isozymes, CA IX and XII, in nomal mucosa of large intestine and in colorectal tumors. Dig Dis Sci 2001:46:2179-86.

34. Chiang WI, Chu SC, Yang SS, Li MC, Lai JC, Yang SF, Chiou HI, Hsich. YS. The abcrant expression of cyrosolic carbonic anhydrase and its clinical significance in human non-small cell lung cancer. Cancer Lett 2002;188:199-205.

35. Unno M, Yonekura H, Nakagawara K, Watanabe T, Miyashita H, Morizumi S, Okamoto H, Itoh T, Teraoka H. Structure, chromosomal localization, and expression of mouse reg genes, reg 1 and reg II. A novel type of reg gene, reg II, exists in the mouse genome, J Biol Chem 1993;268:15974 82. 
36. Narushima Y, Unno M, Nakagawara K, Moti M, Miyashita H, Suruki Y, Noguchi N, Takasawa S, Kumagai T, Yonckura H, Okamoto H. Structure, chromosomal localization and expression of mouse genes encoding type III Reg, Reglll aipha, RegllI beta, Reglll gamma. Gene 1997;185:159. 68.

37. Graf R, Schiesset M, Scheele GA, Marquardt K, Frick TW, Ammann RW, Bimmler D. A family of $16-\mathrm{kDa}$ pancreatic secretory stress proteins form highly organized fibrillar structures upon tryptic activation. J Biol Chem 2001:276:21028-38.

38. Macadam RC, Sarela AI, Farmery SM, Robinson PA, Markham AF, Guillou PJ. Death from carly colorectal cancer is predicted by the presence of transcripts of the REG gene family. Br J Cancer 2000;83:188-95.

39. Hatefi $Y$. The mitochondrial electron transport and oxidative phosphotylation system. Annu Rev Biochem 1985;54:1015-69.

40. Sandri G. Wojtczak L Ernster L. Cation-dependent reassembly of F0F1-ATPase in submitochondrial particles: evidence for a binding site for $\mathrm{F} 1$ on $\mathrm{FO}$ in the absence of $\mathrm{F} 6$ and oligomycin sensitivity-conferring protein. Arch Biochem Biophys 1985,239:595-602.

41. Duszynski J, Dupuis A, Lux B, Vignais PV. Spectral properties of fluorescent derivatives of the oligomycin sensitivity conferring protein and analysis of their interaction with the $\mathrm{F} 1$ and $\mathrm{F} 0$ sectors of the mitochondral ATPase complex. Biochemistry 1988:27:6288-96.

42 Harrington WF, Rodgers ME. Myosin. Annu Rey Biochem 1984;53:35-73.

43. Adelstein RS, Eisenberg E. Regulation and kinetics of the actin-myosin-ATP interaction. Annu Rev Biochem 1980;49:921-56.

44. Hartshorne DJ. Phosphorylation of mysosin and the regulation of smooth muscle actomyosin. In: Dowben RM, Shay JW, eds. Cell and Muscle motility II. New York: Plenum Publishing Corp., 1982:188-220.

45. Cummings JH, Branch W, Jenkins DJ, Southgate DA, Houston H, James WP. Colonic response to dietary fibre from carrot, cabbage, apple, bran. Lancet 1978;1:5-9.

46. Wrick KI, Robertson JB, Van Soest PJ, Lewis BA, Rivers JM, Roe DA, Hackler LR. The influence of dietary fiber source on human intestinal transit and stool output. J Nutr 1983;113:1464-79. 



\title{
Chapter 5
}

\section{Gene expression modulation by specific vegetables in colon mucosa of C57BL6 female mice}

Submitted

\author{
S.G.J. van Breda' \\ E. van Agen' \\ S. van Sanden ${ }^{2}$ \\ T. Burzykowski² \\ J.C.S. Kleinjans! \\ J.H.M. van Delft ${ }^{1}$
}

'Department of Health Risk Analysis and Toxicology, Mastricht University, Maastricht, The Netherlands ${ }^{2}$ Center for Statistics, Limburg University Centre, Diepenbeck, Belgium 


\section{Abstract}

Consumption of cruciferous vegetables, carrots and onions has been shown to exhibit protective effects against colorectal cancer (CRC). The evidence for legumes is less clear, but promising. In a previous study, the combined effect of these four vegetables on gene expression changes in colon mucosa from female C57BL6 mice was examined. The aim of the present study was to investigate the contribution of these four individual vegetables on gene expression, to give more insight into their role in genetic mechanisms underlying CRC prevention. Female C57BL6 mice were randomly divided into five dietary groups (6 mice per group). Each group was fed a different diet, containing no vegetables, cauliflower, carrots, peas or onions, for two weeks. Colonic mucosal cells were collected after sacrifice. Total RNA was isolated and microarray technology was used to measure the expression levels of 602 genes simultaneously. Eighteen genes were significantly modulated by one or more of the specific vegetables. Ten of these are known to be involved in CRC and seven out of these ten were affected in such a way that CRC protective mechanisms may be stimulated. The four individual vegetables seem to have about the same potential of modulating genes in favor of CRC protection. Eleven similar genes were modulated in the present study as in the previous mixture study and for seven of them the modulation by the mixture could be explained by the effect of a particular vegetable. These similarly modulated genes are of particular interest because they are consistently affected and could play an important role in CRC prevention by vegetables. 


\section{Introduction}

In Western countries, colorectal cancer (CRC) is one of the most common malignant diseases, accounting for 15.3 percent of all new cancer cases. Although treatment of CRC continues to improve, large-bowel cancer remains a major cause of illness and death.'

Substantial evidence from epidemiological, clinical, and laboratory studies shows that risk of CRC cancer is closely related to diet and other lifestyle factors. Decreased intake of dietary fat, increased intake of vegetables and fruit and high physical activity is associated with decreased risk of developing CRC. The most consistent observation is that vegetable consumption is inversely associated with CRC risk. ${ }^{17}$

Particularly, carrots, cruciferous and allium vegetables have been shown to exhibit protective effects. The evidence for legumes, like beans and peas, is promising, but inconclusive. $1,5,7$ These vegetables may be protective against CRC by providing the colon with a range of anticarcinogenic factors. These compounds act via various different proposed mechanisms, such as modulation of phase-I and -II metabolizing enzymes, induction of cell differentiation, inhibition of cell proliferation and scavenging of free radicals. $3,5,7.11$

Although during the last decade an intensified investigation of the effects of diet on the colon has taken place ${ }^{12.18}$, the genetic pathways through which vegetable components exert their effects are mostly unknown. Furthermore, the number of experimental studies in which the effect of whole vegetables, instead of individual micronutrients or other bioactive compounds are examined, is limited. By investigating the effect of whole vegetables, the biological availability of the compounds from the food matrix and their possible interactions can be taken into account.

In a previous study, the effect of a diet providing different doses of a vegetable mixture consisting of cauliflower, carrots, peas and onions on gene expression changes in colon mucosa of female C57BL6 mice was examined by means of cDNA microarray technology. (Chapter 3) The results of that study indicated that a diet with a high content of vegetables positively modulates genes involved in protective mechanisms at various stages of CRC development. In the present study, the contribution of the individual vegetables on gene expression changes in colon mucosa of female C57BL6 mice is investigated. This will provide more information about the role of these specific vegetables in the genetic mechanisms underlying CRC prevention. 


\section{Materials and Methods}

\section{Animals and Diets}

Eight-week-old female C57BL6 mice (Charles River Laboratories, Lyon, France) were randomly assigned to one of five dietary groups, each consisting of six animals. During 7 days of acclimatization, all the animals received the standard control diet. Next, each group was fed one of five different diets, containing no vegetables (referred to as control diet), cauliflower, carrots, peas or onions respectively, for a period of two weeks. This study succeeds a previously conducted mouse study in which the effect of a diet containing different doses of a vegetable mixture consisting of cauliflower (30\% wet wt), carrots $(30 \%$ wet wt), peas (30\% wet wt), and onions (10\% wet wt) was investigated. (Chapter 3 ) The diet in the highest vegetable dose group contained $40 \%$ of this vegetable mixture. The amount of vegetables used in the present study is equal to the amount of these specific vegetables in the $40 \%$ vegetable dose group. In the vegetable mixture study, most of the statistical differences were observed between the highest vegetable dose group (40\%) and one of the other diet groups. Diets were refreshed every two days and provided as powdered feed. The $20 \%$ casein reference diet (Hope Farms, Woerden, The Netherlands) served as the basal diet for the five different diets. The vegetables were from the same batch used in the previous study. This batch was purchased as a single batch at the supermarket and separately cooked under household conditions. After freezing $\left(-20^{\circ} \mathrm{C}\right)$, the vegetables were lyophilized, ground and combined. Before the vegetables were mixed with the basal diet, they were analyzed for macronutrient content (Hope Farms). The vegetables added to the basal diet were at the expense of carbohydrates; therefore diets were supplied with dextrose/cerelose and cellulose (dicacel) (Hope Farms), resulting in similar energy densities. No antioxidants or preservatives were added. After preparation, the diets were stored at $-20^{\circ} \mathrm{C}$ in air-closed plastic bags until use. The composition of the different diets is given in Table 1.

The animals were maintained in the laboratory animal care facilities at the University of Maastricht under controlled environmental conditions (temperature $21 \pm 1$ ${ }^{\circ} \mathrm{C}$, relative humidity $50 \% \pm 10,12$-hr light/dark cycle). Body weights of the mice were recorded weekly. The study was approved by the Institutional Committee of Animal Experimentation of Maastricht University.

\section{Tissue Sampling}

Mice were sacrificed by bleeding the vena cava inferior under Nembutal (Sanofi Sante, Maasluis, the Netherlands) anesthesia. Nembutal was administered subcutaneously in the neck at a dose of $60 \mathrm{mg} / \mathrm{kg}$ body weight. The large intestine was removed and placed on a box, which was kept at $4{ }^{\circ} \mathrm{C}$. After removing the rectum, the colon was opened longitudinally with fine scissors and mucus and faeces were removed. Colonic mucosal 
cells were incubated in Trizol ${ }^{\mathrm{TM}}$ (GIBCO Life Technologies) for 3 minutes and scraped off the muscle layer with the edge of a sterile glass slide. Cells were transferred into $800 \mu \mathrm{l}$ Trizol ${ }^{\mathrm{TM}}$, homogenized by resuspendation and stored at $-80^{\circ} \mathrm{C}$ until total RNA isolation.

Table 1. Composition of the five diets in $\mathrm{g} / \mathrm{kg}$.

\begin{tabular}{|c|c|c|c|c|c|}
\hline Ingredients & $\begin{array}{c}\text { Control } \\
\text { diet }^{1} \\
\end{array}$ & $\begin{array}{c}\text { Cauliflower } \\
\text { diet }^{2} \\
\end{array}$ & $\begin{array}{c}\text { Carrots } \\
\text { diet }^{2} \\
\end{array}$ & $\begin{array}{l}\text { Peas } \\
\text { diet }^{2} \\
\end{array}$ & $\begin{array}{c}\text { Onions } \\
\text { diet }^{2}\end{array}$ \\
\hline Cerelose/Dextrose & 515 & 451 & 452 & 303 & 488 \\
\hline DL-Methionine & 2 & 2 & 2 & 2 & 2 \\
\hline $\mathrm{NaCl}$ & 3 & 3 & 3 & 3 & 3 \\
\hline Choline CL $50 \%$ & 4 & 4 & 4 & 4 & 4 \\
\hline Soya oil & 50 & 50 & 50 & 50 & 50 \\
\hline KCI & 7 & 7 & 7 & 7 & 7 \\
\hline $\mathrm{KH}_{2} \mathrm{PO}_{4}$ & 7 & 7 & 7 & 7 & 7 \\
\hline $\mathrm{MgO}$ & 2 & 2 & 2 & 2 & 2 \\
\hline $\mathrm{CaHPO}_{4} 2 \mathrm{H}_{2} \mathrm{O}$ & 13 & 13 & 13 & 13 & 13 \\
\hline $\mathrm{CaCO}_{3}$ & 10 & 10 & 10 & 10 & 10 \\
\hline Com Starch & 100 & 100 & 100 & 100 & 100 \\
\hline Dicacel/Cellulose & 77 & 71 & 67 & 63 & 73 \\
\hline Casein & 200 & 200 & 200 & 200 & 200 \\
\hline $\mathrm{MgSO}_{4} \cdot 7 \mathrm{H}_{2} \mathrm{O}$ & 4 & 4 & 4 & 4 & 4 \\
\hline $\begin{array}{l}\text { Standard vitamin } \\
\text { premix }\end{array}$ & 3 & 3 & 3 & 3 & 3 \\
\hline $\begin{array}{l}\text { Standard micro- } \\
\text { nutrient premix }\end{array}$ & 3 & 3 & 3 & 3 & 3 \\
\hline Vegetables ${ }^{3}$ & 0 & 70 & 73 & 226 & 31 \\
\hline
\end{tabular}

'Control diet is the basal $20 \%$ casein reference diet.

In the vegetable diets, the basal diet was adjusted for cerelose/dextrose and dicacel/cellulose resulting in samilar energy densities for all diets.

wet weight: cauliflower $1.8 \mathrm{~kg}$, carrots $1.8 \mathrm{~kg}$ peas $1.8 \mathrm{~kg}$, onions $0.6 \mathrm{~kg}$.

\section{Total RNA Isolation and cDNA Probe Synthesis}

Total RNA was extracted according to the manufacturer's instructions. The RNeasy® Mini Kit (Qiagen) together with a DNase treatment was used to purify total RNA from salts and residual DNA. Quantity of each RNA sample was measured by a spectrophotometer and varied from 20 to $90 \mu \mathrm{g}$ per mouse. Integrity was determined by a Bioanalyzer (Agilent Technologies Netherlands B.V., The Netherlands). All samples contained intact total RNA with an $\mathrm{rRNA}$ ratio $(28 \mathrm{~S} / 18 \mathrm{~S})>1.5$.

The $0 \%$ vegetable diet group was considered as the control group; the vegetable diet groups were referred to as the test groups. Three RNA pools per diet group were prepared by pooling equal amounts of total RNA from two mice. Cyanine 3- (Cy3) and 
Cyanine 5- (Cy5) labeled cDNA probes were prepared using $10 \mu \mathrm{g}$ total RNA from each pool, by the method of Hasseman et al. ${ }^{19}$

\section{¿DNA Microarray Preparation}

This study is part of a project that investigates the effects of vegetables on gene expression in colon mucosa of humans and mice. For the human study, the expression levels of genes in colon mucosa were measured using the PHASE-1 Microarray Human-600 (PHASE-1 Molecular Toxicology, Santa fee, USA). A mouse cDNA microarray was constructed based on the genes present on the PHASE-1 Microarray Human-600. These genes represent a dedicated set of biologically relevant gene sequences involved in inflammation, DNA damage and repair, oxidative stress, cell signaling, cell proliferation, metabolism, transcription and apoptosis. Selection of mouse cDNA clones, amplification of cDNA inserts by PCR, verification and purification of PCR products and spotting of the microarrays was carried out as described in Chapter 3 (Section Materials and Methods; cDNA Microarray Preparation).

\section{cDNA Microarray Hybridizations}

For the hybridizations, within each diet group three pools were formed, each consisting of equal amounts of total RNA of two or three mice. By creating three pools per diet group instead of one, biological variability can be taken into account, what allows for a broader generalizability of the results. For each of the three sets of pools from different diet groups, a reference hybridization design was constructed. ${ }^{20}$ In this design, each test group is compared with the same pool from control group. Cy3 and Cy5 labeled cDNA probes of the test and control group were mixed and hybridized to the cDNA microarray by the method of Hasseman et al. ${ }^{10}$ The reference design was chosen because it allows for the same precision of all comparisons of test groups against the control. In order to remove potential bias to dye effects, a second microarray experiment was carried out for each couple, in which the dyes were switched (flip-dye experiment). In total, 24 cDNA microarray hybridizations were performed. Slides were scanned on a GMS 418 Array Scanner (Affymetrix, High Wycombe, UK). The images obtained (resolution 10 micron; 16 bit tiff image) were processed with ImaGene 5.0 software (Biodiscovery, Los Angeles, USA) to measure mean signal intensities for spots and local background.

\section{DN A Micmama Data Processing and Statistical Analysis}

The data were analyzed using ANOVA models ${ }^{21}$, without background correction and using base-2 logarithmic transformation of the measured intensities. Due to computational limitations, the models were fit in two stages. The models included a normalization step, taking into account both the global (across-genes) and local (gene-specific) normalization. ${ }^{22}$ All pairwise differences between the five diet groups were examined. For each gene, the 
Tukey procedure was used to correct for multiple comparisons. ${ }^{2}$ To control the overall (across genes) probability of false positive findings, at around 5\%, a p-value $<0.0001$ was considered to indicate statistical significance of an individual pairwise comparison. The critical values for the Tukey procedure were selected using an empirical distribution obtained by bootstrap. ${ }^{24}$

\section{Real-time RT-PCR}

To verify the cDNA microarray results, 14 gene expression differences, representing six genes which were responsive to vegetables, were analyzed by real-time RT-PCR, as described previously in Chapter 3 (Section Materials and Methods; Real-time RT-PCR). A list of validated genes and the normalization gene and their primers is given in Table 2.

\section{Additional Statistical Anahysis}

Statistical analysis of bodyweight of the mice was carried out using SPSS version 6.1.1 for Macintosh. Data were analyzed by means of analysis of variance followed by Student's $t$ test. $A$ two-sided $\mathrm{p}$-value $<0.05$ was considered to indicate statistical significance.

\section{Results}

Mean body weights per week per group are presented in Table 3. Prior to the start of the intervention, all animals received the control diet (Week 0). At the end of this week, there were no statistically significant differences in mean body weights between the groups. After the intervention, mean body weights were significantly increased within each group $(\mathrm{P}<0.05)$, but no significant differences between the groups were observed. Because the diets are isocaloric and weight gain was similar between the groups, these results indicate that there was no difference in food intake and that the diets did not affect normal growth.

\section{Table 3. Mean body weights (g) per week per group. ${ }^{\prime}$}

\begin{tabular}{|lccc|}
\hline & Week 02 & Week 13 & Week 2' \\
\hline Control group (C) & $17.6 \pm 0.9$ & $18.7 \pm 1.0$ & $19.4 \pm 1.4^{4}$ \\
Cauliflower group (T1) & $18.3 \pm 0.6$ & $19.5 \pm 0.6$ & $20.7 \pm 0.5^{4}$ \\
Carrots group (T2) & $17.9 \pm 0.3$ & $19.7 \pm 0.7$ & $20.2 \pm 0.8^{4}$ \\
Peas group (T3) & $18.2 \pm 0.6$ & $19.3 \pm 1.1$ & $20.3 \pm 1.1^{4}$ \\
Onions (T4) & $18.0 \pm 0.8$ & $18.9 \pm 0.7$ & $19.6 \pm 0.7^{4}$ \\
\hline
\end{tabular}

'Body weights are expressed as mean $\pm \mathrm{SE}$.

Aeclimatization week, animals recerved the control diet.

Intervention period.

'Statisrics: amalysis of variance followed by Student's t-test. $P<0,05$; body weight significantly higher compared to week 0 . 
Table 2. Primer sequences of the validated genes and the 185 normalization gene.

\begin{tabular}{|c|c|c|c|}
\hline $\mathrm{AC} \# 1$ & $\begin{array}{l}\text { Gene Name by NCBI } \\
\text { (Abbreviation) }{ }^{2}\end{array}$ & Forward Primer & Reverse Primer \\
\hline BCo 073773 & $\begin{array}{l}\text { Translation elonganon factor EE-1 } \\
\text { alpha-1 chain (EFHU1) }\end{array}$ & 5'-GGCAAGCCCATGTGTGTTG-3' & 5'-CCTCATGTCACGAACAGCAAA-3' \\
\hline BC,07306? & $\begin{array}{l}\text { Heat shock } 60 \mathrm{kDa} \text { protein ! } \\
\text { (chaperonin) (HSPD1) }\end{array}$ & 5'-CCGAAGACGTTGACGGAGAA-3' & 5'-TGCCACAACCTGAAGACCAA-3' \\
\hline A1152753 & $\begin{array}{l}\text { Caspase 4, apoptosis-related cysteine } \\
\text { protease (CASP4) }\end{array}$ & 5'-CGGGCAACCTTGACGAGAT-3' & 5'-TTCAAGCTGAAGAAATGAGATTCAGTT-3' \\
\hline $\mathrm{BG}(\mathrm{n} 5221$ & $\begin{array}{l}\text { Omithine decarboxylase, structural } \\
\text { (ODC) }\end{array}$ & 5'-CAACATCATTGCCAAARAAACC-3' & 5'-TGTTCATTTGACTCATCTTCATCGT-3' \\
\hline W88005 & $\begin{array}{l}\text { Cyclin-dependent kinase inhibitor iA } \\
\text { (p21) (CDKNIA) }\end{array}$ & 5'-GCAGATCCACAGCGATATCCA-3' & 5'-GGTCGGACATCACCAGGATT-3' \\
\hline $\mathrm{BC}, 074600$ & $\begin{array}{l}\text { Omithine aminotransferase (OAT) } \\
18 \mathrm{~S}\end{array}$ & $\begin{array}{l}5^{\prime} \text {-GTGAGAGGGAAAGGGTTGCTAA-3' } \\
5^{\circ} \text {-AGTCCTGCCCTTITACACA-3. }\end{array}$ & $\begin{array}{l}\text { 5'-CAGGCACACCTTCCAAGCAT-3' } \\
5^{\prime} \text {-GATCCGAGGGCCTCACTAAAC-3. }\end{array}$ \\
\hline
\end{tabular}

'ACH: Genlank accession numbers of the $\mathrm{CDN}$ A fragments present on the microarrays.

AC.BI (Nathonal Center for Biotechnology Information): http://www.ncbinimnih.gov/. 
The expressions of 602 genes were measured simultaneously by means of cDNA microarrays. Microarray data analysis compared the levels of expression between the diet groups and tested whether these differences were significant. Thereby, a significant difference in gene expression was found for 34 diet group comparisons, representing 18 genes. These genes, their matching comparisons and the differences are listed in Table 4.

\section{Table 4. Differentially expressed genes.}

\begin{tabular}{|c|c|c|c|c|}
\hline Ac\#! & Gene Name by NCBI (Abbreviation) ${ }^{2}$ & Comparison & $\begin{array}{c}\text { Difference cDNA } \\
\text { microarray }\end{array}$ & $\begin{array}{l}\text { Difference } \\
\text { RT-PCR }\end{array}$ \\
\hline \multirow[t]{2}{*}{ BG073773 } & Translation clongation factor EF-1 alpha-1 & T1-C & $-0.23 \pm 0.06$ & $-1.46 \pm 0.18$ \\
\hline & chain (EFHU1) & $\mathrm{T} 2 \cdot \mathrm{C}$ & $-0.19 \pm 0.07$ & $-0.82 \pm 0.17$ \\
\hline BG074767 & $\begin{array}{l}\text { Topoisomerase (DNA) II alpha } 170 \mathrm{kDa} \\
\text { (TOP2A) }\end{array}$ & $\mathrm{T} 4 \mathrm{C}$ & $-1,05 \pm 0,10$ & \\
\hline \multirow[t]{2}{*}{ BG076302 } & Interleukin 18 (interferon-gamma-inducing & T3-C & $0.93 \pm 0.11$ & \\
\hline & factor) (1L.18) & T3-T2 & $1.11 \pm 0.15$ & \\
\hline \multirow[t]{4}{*}{ BC;073067 } & $\begin{array}{l}\text { Heat shock 60kDa protein } 1 \text { (chaperonin) } \\
\text { (HSPD1) }\end{array}$ & $\mathrm{T} 1 \cdot \mathrm{C}$ & $-0.54 \pm 0.09$ & $-2.38 \pm 0.15$ \\
\hline & & $\mathrm{T} 2 \mathrm{C}$ & $-0.55 \pm 0.09$ & $-1.72 \pm 0.21$ \\
\hline & & T3. C & $-0.57 \pm 0.19$ & $-1.72 \pm 0.15$ \\
\hline & & $\mathrm{T} 4 \mathrm{C}$ & $-0.57 \pm 0.08$ & $-1.40 \pm 0.15$ \\
\hline \multirow[t]{2}{*}{ A 396890} & BCI2/adenovirus E1B 19kDa-interacting & $\mathrm{T} 4 \mathrm{C}$ & $-0.24 \pm 0.05$ & \\
\hline & protein 1 (BNIP1) & $\mathrm{T} 2 \cdot \mathrm{C}$ & $-0.19 \pm 0.05$ & \\
\hline BG065603 & $\begin{array}{l}\text { Cytochrome c oxidase subunit IV isoform } 1 \\
\text { (COX411) }\end{array}$ & $\mathrm{T} 1 \cdot \mathrm{C}$ & $0.22 \pm 0.06$ & \\
\hline \multirow[t]{3}{*}{ W88005 } & Cyclin-dependent kinase inhibitor $1 \mathrm{~A}\left(\mathrm{p}^{21}\right)$ & T1-C & $0.68 \pm 0.09$ & $0.44 \pm 0.17$ \\
\hline & $(\mathrm{CDKN} 1 \mathrm{~A})$ & $\mathrm{T} 2 \cdot \mathrm{C}$ & $0.72 \pm 0.09$ & $1.00 \pm 0.40$ \\
\hline & & T3-C & $0.69 \pm 0.09$ & $0.57 \pm 0.17$ \\
\hline \multirow[t]{3}{*}{ BG065221 } & Ornithine decarboxylase, structural (ODC) & T1-C & $-0.52 \pm 0.06$ & $-1.73 \pm 0.18$ \\
\hline & & $\mathrm{T} 2 \mathrm{C}$ & $-0.51 \pm 0.06$ & $-1.26 \pm 0.21$ \\
\hline & & $\mathrm{T} 2 \mathrm{~T} 3$ & $-0.44 \pm 0.08$ & \\
\hline BG064900 & Stearoyl-Cocnzyme A desaturase 2 (SCD2) & $\mathrm{T} 4 \mathrm{C}$ & $0.32 \pm 0.07$ & \\
\hline BG063870 & $\begin{array}{l}\text { Beta actin, cytoplasmic (ACTB); clone } \\
\text { H3011G02 }\end{array}$ & $\mathrm{T} 1-\mathrm{C}$ & $0.74 \pm 0.11$ & \\
\hline \multirow[t]{3}{*}{ Al152753 } & Caspase 4 , apoptosis-related cysteine protease & $\mathrm{T} 1-\mathrm{C}$ & $0.77 \pm 0.12$ & $1.31 \pm 0.19$ \\
\hline & (CASP4) & $\mathrm{T} 1-\mathrm{T} 4$ & $1.14 \pm 0.16$ & \\
\hline & & T2.T4 & $0.96 \pm 0.16$ & \\
\hline BG063771 & $\begin{array}{l}\text { Glyceraldehyde-3-phosphate dehydrogenase } \\
\text { (GAPDH);clone H303E11 }\end{array}$ & $\mathrm{T} 1-\mathrm{T} 4$ & $0.46 \pm 0.10$ & \\
\hline \multirow[t]{4}{*}{ BC:074606 } & $\begin{array}{l}\text { Ornithine aminotransferase (OAT); clone } \\
\text { H313B06 }\end{array}$ & $\mathrm{T} 1-\mathrm{C}$ & $-0,48 \pm 0,09$ & $-2.95 \pm 0.10$ \\
\hline & & T2-C & $-0.78 \pm 0.09$ & $-0.70 \pm 0.22$ \\
\hline & & $\mathrm{T} 2 \mathrm{~T} 3$ & $-0.59 \pm 0.11$ & \\
\hline & & T2-T4 & $-0.58 \pm 0.12$ & \\
\hline
\end{tabular}


Table 4. (Continued)

\begin{tabular}{|llccc|}
\hline Ac\#1 & Gene Name by NCBI (Abbreviation) & Comparison & $\begin{array}{c}\text { Difference cDNA } \\
\text { microarray }\end{array}$ & $\begin{array}{c}\text { Difference } \\
\text { RT-PCR }^{4}\end{array}$ \\
\hline BG063081 & Thymosin, beta 10 (TMSB10) & T4-C & $-0.79 \pm 0.09$ \\
BG075853 & Selenoprotein P, plasma 1 (SEPP1) & T4-C & $0.68 \pm 0.10$ \\
AA500809) & Peripheral myelin protein (PMP22) & T1-C & $0.69 \pm 0.09$ \\
& & T2-C & $0.70 \pm 0.09$ \\
BG086656 & Aldehyde dehydrogenase family 1, subfamily A1 & T1-C & $-0.80 \pm 0.11$ \\
& (ALDH1A1) & & \\
BG064142 & Hypoxia inducible factor 1, alpha subunit & T4-C & $0.94 \pm 0.09$ & \\
& (HIF1A) & & \\
\hline
\end{tabular}

ICA: GenBank accession numbers of the cDNA fragments present on the microarrays.

NCBI (National Center for Biotechnoiogy Information): hrtp://www.ncbi.nlm.nih.gov/.

Fistimated difference in mean loge-transformed intensity \pm SE. (C: control group; T1: cauliflower group; T2: carrots group; T3: peas group: T4: onions group).

Normalized difference in mean Jog-transformed intensity \pm SE. Fourteen gene differences were validated with RT-PCR Empty cells indicate gene differences which were not taken into account.

In Figure 1, the estimated differences of these genes per vegetable group as comparcel to the control group (set to $O$ ) are depicted. Furthermonc, a schematic overviow of the genes with respect to the particular vegetables, by which they are differentially expressed, is visually described in a Venn diagram (Figure 2).

Heat shock $60 \mathrm{kDa}$ protein 1 (chaperonin) (HSPD1) is the only gene the expression of which was statistically significantly modulated in all vegetable groups compared to the control group. Next to HSPD1, six genes were affected by more than one, but not all vegetable groups, i.e. Translation elongation factor EF-1 alpha-1 chain (EFHU1), Ornithine decarboxylase, structural (ODC), Caspase 4, apoptosis-related cysteine protease (CASP4), Ornithine aminotransferase (OAT), Peripheral myelin protein (PMP22), all by cauliflower and carrots; Cyclin-dependent kinase inhibitor 1A (p21) $(\mathrm{CDKN} 1 \mathrm{~A})$ in the cauliflower-, carrots- and peas group; and BCl.2/adenovirus E1B $19 \mathrm{kDa}$-interacting protein 1 (BNIP1) by carrots and onions. Ten genes were modulated in one specific vegetable group; onions had an effect on the expression of Topoisomerase (DNA) $I 1$ alpha 170 kDa (TOP2A), Stearoyl-Coenzyme A desaturase 2 (SCD2), Thymosin, beta 10 (TMSB10), Selenoprotein P, plasma 1 (SEPP1) and Hypoxia inducible factor 1, alpha subunit (HIF1A); peas modulated Interleukin 18 (interferon-gamma-inducing factor) (11.18) gene expression; and cauliflower affected the expression of Cytochrome c oxidase subunit IV isoform 1 (COX4I1), Beta actin, cytoplasmic (ACTB) and Aldehyde dehydrogenase family 1, subfamily A1 (ALDH1A1). No genes were modulated solely by the carrots. The expression of Glyceraldehyde-3-phosphate dehydrogenase (GAPDH) was statistically significantly different between the cauliflower group and the onions group, but 
no difference was observed compared to the control group. Most genes (10) were modulated in the cauliflower group and least (3) in the peas group.
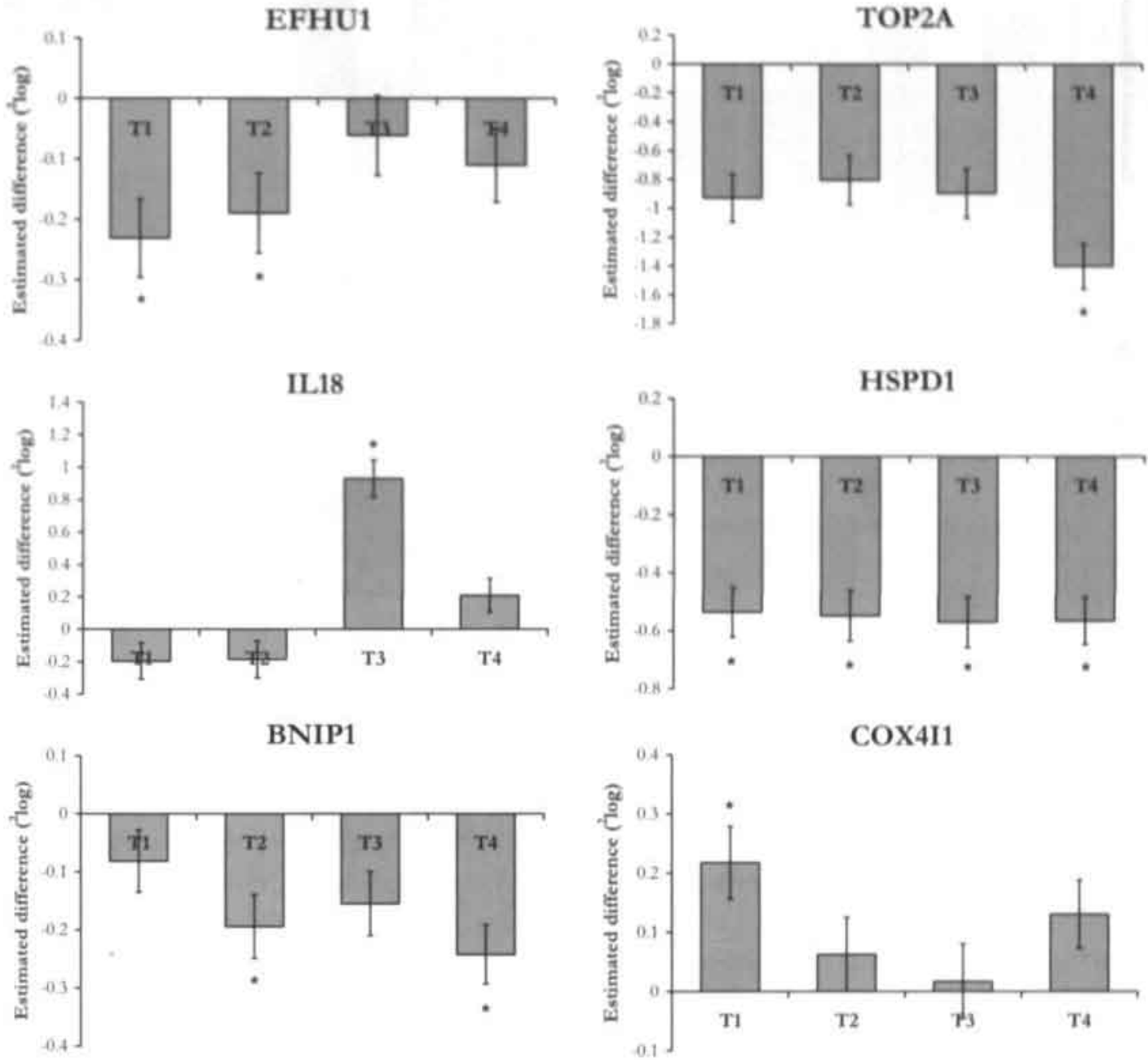

Figure 1. Estimated difference in mean Fog-transformed intensity \pm SE per diet group (x-axis) (T1: cauliflower group; T2: carrots group; T3: peas group; T4: onions group) for all identified differentially expressed genes from Table 4. The control diet group intensity (set to 0 ) serves as the reference. EFHU1: Translation elongation factor EF-1 alpha-1 chain; TOP2A: Topoisomerase (DNA) II alpha $170 \mathrm{kDa}$; II.18: Interleukin 18 (interferon-gamma-inducing factor); HSPD1: Heat shock 60kDa protean 1 (chaperonin); BNIP1: BCI2/adenovirus E1B 15.Da-interacnng protein 1; COX411: Cytochrome coxidase subunit IV isoform 1: CDKN1A: Cyclin-dependent kinase inhibitor 1A (p21); ODC: Ornuthine decarboxylase, structural; SCD2 Steatoyl-Coenzyme A desarurase 2: ACTB: Beta actin, cytoplasmic; CASP4: Caspase 4, apoptosis-related cysteine protease; GAPDH: Głyceraldehyde-3-phosphate dehydrogenase; OAT: Ornithine aminotransferase; TMSB16): Thymosin, beta 10: SEPP1: Selenoprotein P. plasma 1: PMP22: Perpheral myelin protein: ALDH1 11: Adehyde dehydrovernase family 1. subfamily A1; HIF1A: Hypoxa inducible factor 1, alpha subunit. Astensk indicates sagneficant differences berween diet group and control ( $0^{\circ}$. vegetable det group). Sce Tabie 4 for other stanstical details. 

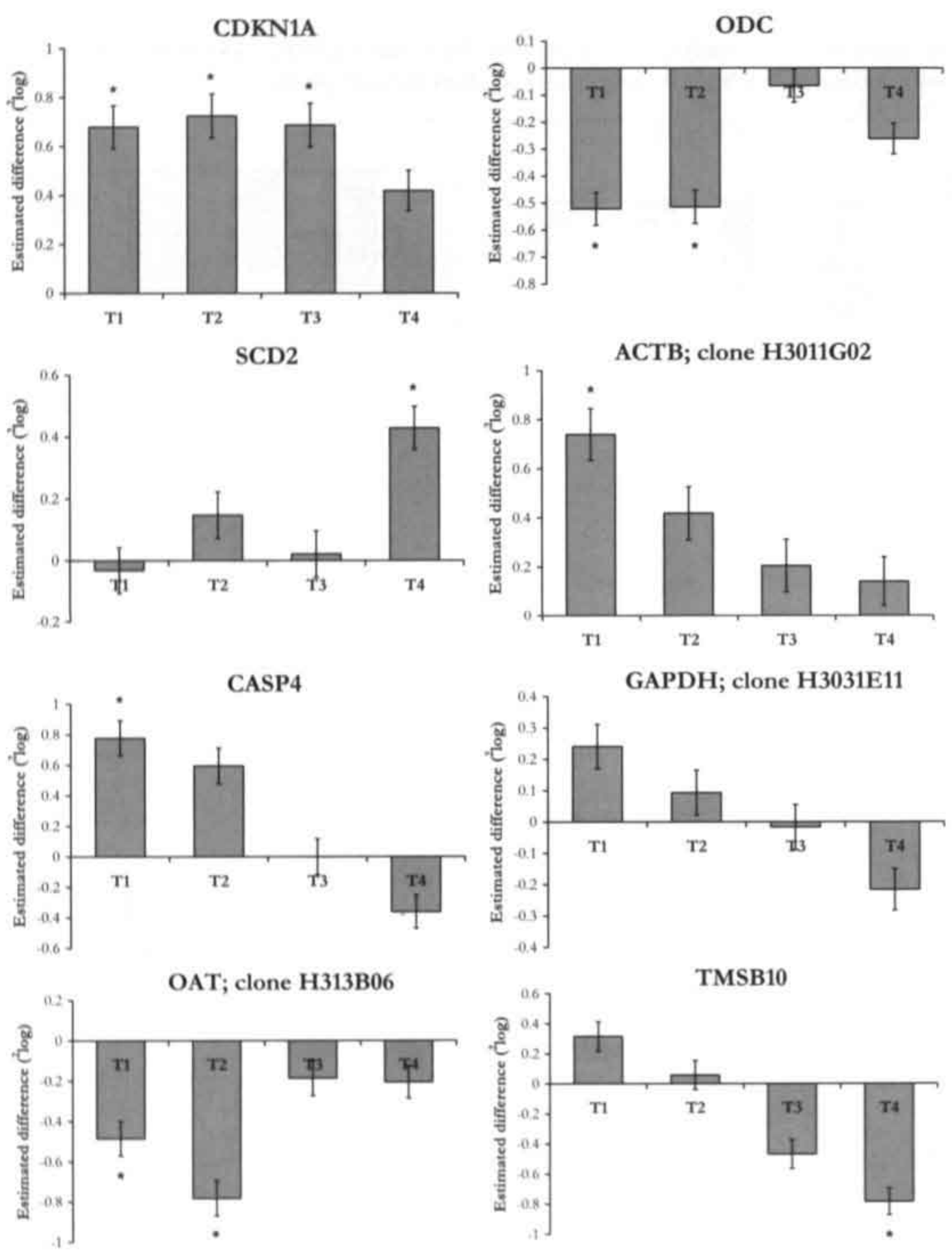

Figure 1. (Continued) 

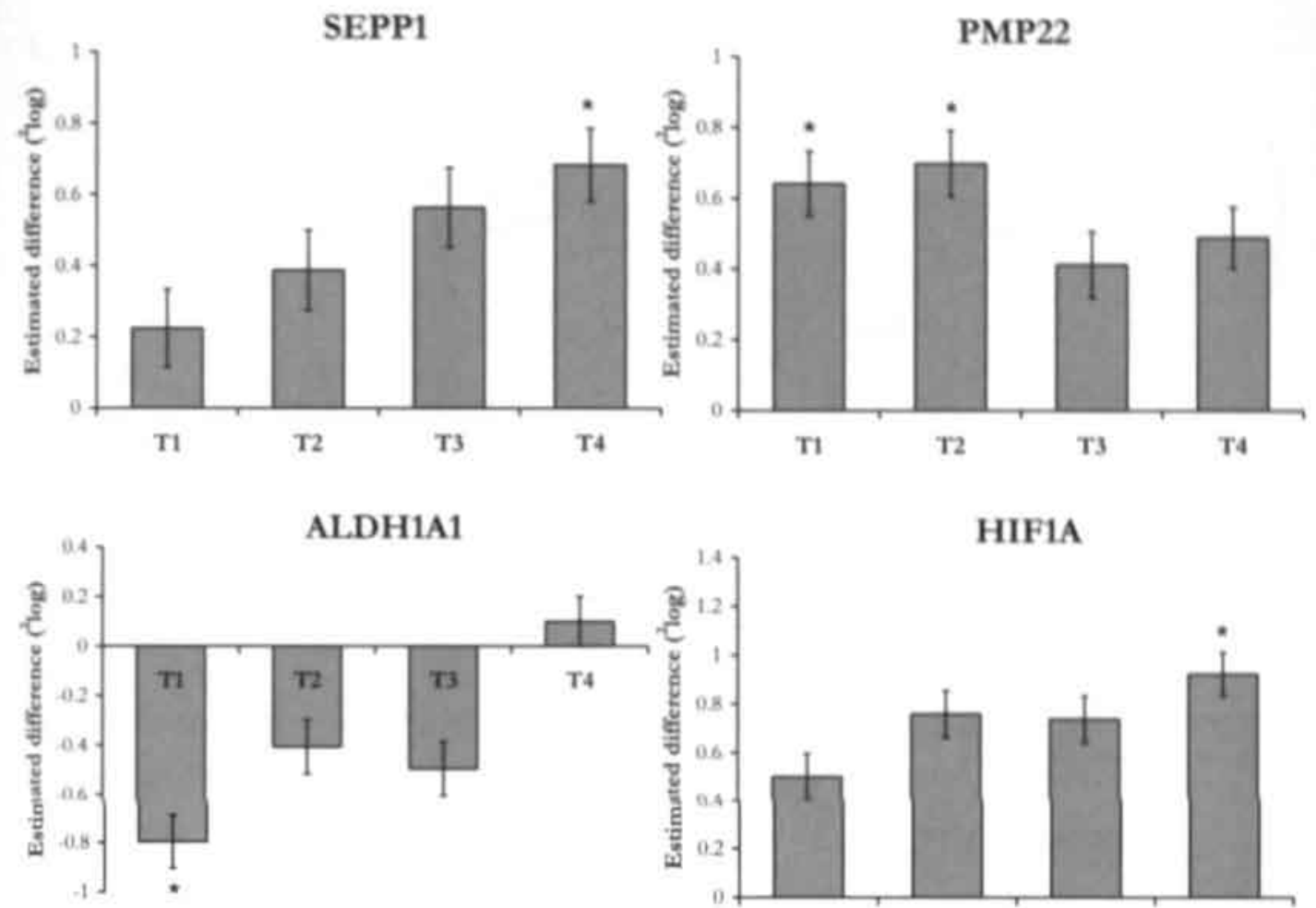

Figure 1. (Continued)

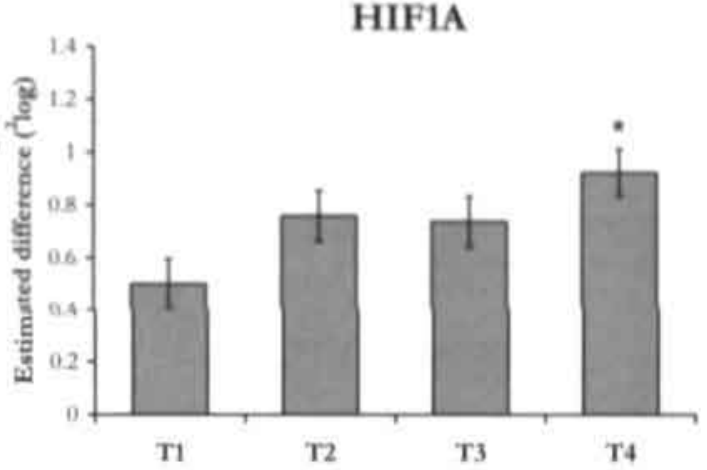

Eleven genes were differentially expressed in the present study as well as in the vegetable mixture study. (Chapter 3; section Results) For seven of these eleven genes, the direction in which the genes were modulated ( $\uparrow$ up- or $\downarrow$ down-) in the current test groups is similar as in the $40 \%$ group of the vegetable mixture study, i.e. EFHU1 ( $\downarrow)$, SCD2 ( $\uparrow$ ), ACTB $(\uparrow)$, CASP4 $(\uparrow$, although in the $40 \%$ group not significantly different compared to control group), OAT $(\downarrow), \operatorname{PMP} 22(\uparrow)$ and HIF1A $(\uparrow)$. Opposite modulation was observed for the three genes TMSB10 ( $\downarrow$ in current study, $\uparrow$ in previous study), ALDH1A1 ( $\downarrow$ in current study, $\uparrow$ in previous study) and SEPP1 ( $\uparrow$ in current study, $\downarrow$ in previous study). For GAPDH, a difference in expression was observed between the cauliflower group $(\uparrow)$ and onions group, but not as compared to the control group, whereas in the vegetable mixture study an up-regulation was observed in the $40 \%$ vegetable dose group as compared to the control group. In Figure 3A-D, for each of the individual vegetable groups of the present study, the genes which are vegetable specific, vegetable mixture specific and overlapping between these two groups are visually described in a Venn diagram. 


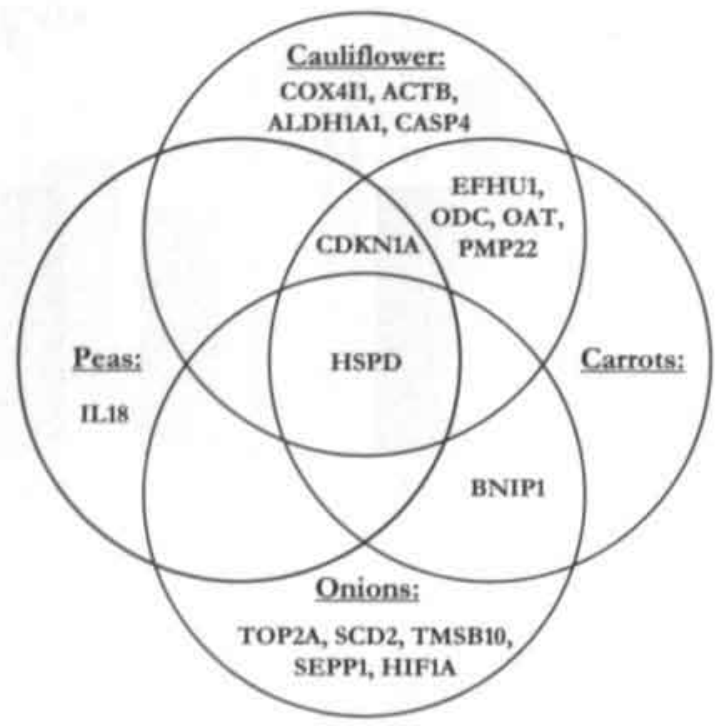

Figure 2. Venn diagram of the significantly differentially expressed genes, with respect to the vegetable group(s) in which they are differentially expressed.

Real-time RT-PCR was used to confirm 14 gene expression differences, representing six genes as responsive to vegetables, identified by cDNA microarray analyses. The results are presented in Table 4 and show in all cases the same direction of up- or down-effect. These data verify the results obtained by cDNA microarray analyses.

A

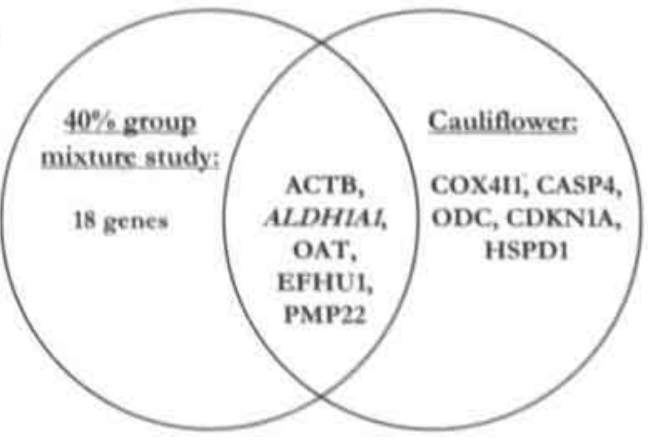

B

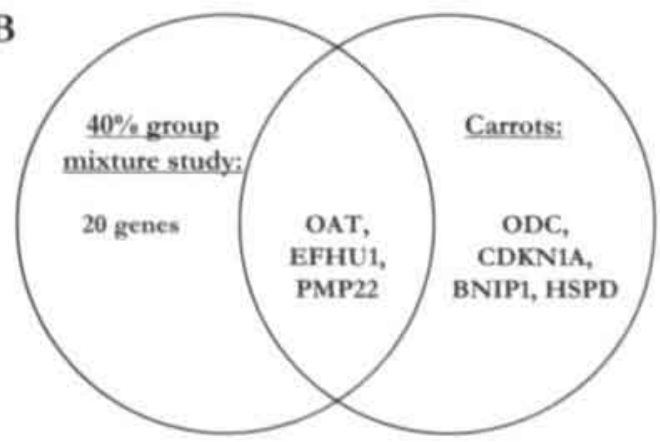

Figure 3. Venn diagram of the significantly differentally expressed genes for each of the individual vegetable groups of the present study and the $40 \%$ group of the previous vegetable mixrure study (Chapter 3). The genes which are vegetable specific, or which are present in the vegetable specific and vegetable mixture groups, are shown. Genes in lalic style are modulated in opposite directions in the individual vegetable group as compared to the $40^{\circ}$. mixrure group. (A) Venn diagram of cauliflower group and $40^{\circ} \mathrm{g}$ group of vegetable mixture. (B) Venn dagram of carrots group and $400^{\circ}$, group of vegetable mixture. (C) Venn dagram of peas group and $40^{\circ}$ i group of vegetable mixture. (D) Venn dagram of onions group and $40^{\circ}$. group of vegetable mixture. 

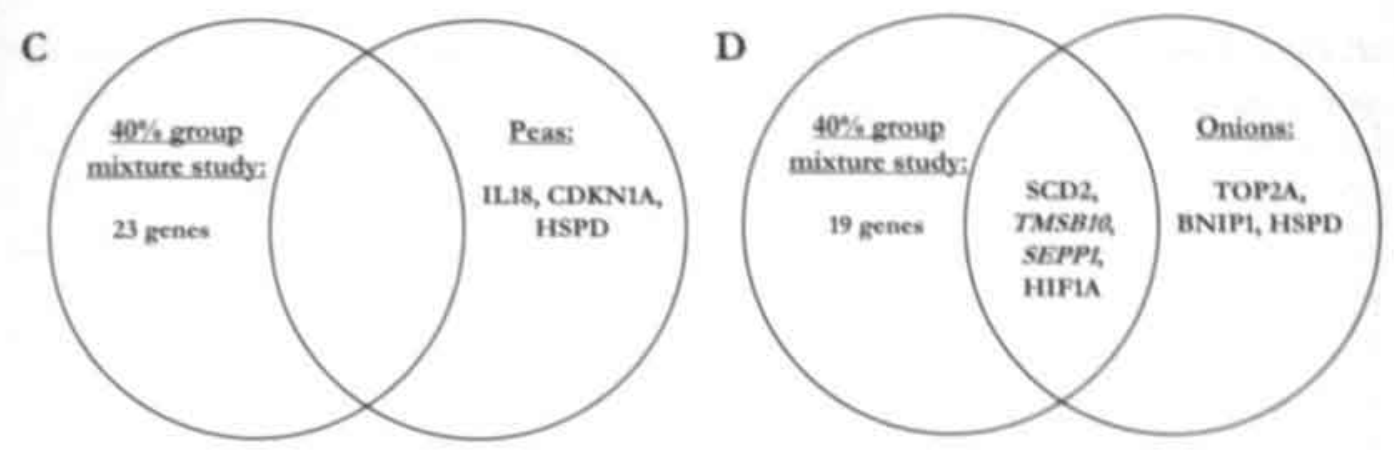

Figure 3. (Contnnued)

\section{Discussion}

In the present study, the contribution of four individual vegetables on gene expression changes in colon mucosa of female C57BL.6 mice is investigated by means of CDNA microarray technology.

For 18 genes a significant difference in gene expression was found for 34 diet group comparisons. Table 5 summarizes the effects on gene expression in relation to possible effects on colon carcinogenesis risk. HSPD1 was the only gene which was significantly modulated (down-regulated) by all vegetables. HSPD1 encodes for a member of the family of chaperones, which can be defined as proteins which assist other proteins to reach their final active form. ${ }^{25}$ Their level of expression is highly increased when a cell is subjected to stress conditions ${ }^{26}$ It could be that the vegetables provide the cell with compounds which maintain homeostasis, thereby reducing the call for stress-induced proteins resulting in their decreased transcription.

Most of the significantly modulated genes ( $\uparrow$ up- or $\downarrow$ down-regulated as compared to control) were in the cauliflower group, i.e. EFHU1 $(\downarrow), \operatorname{COX} 4 \mathrm{I1}(\uparrow), \mathrm{CDKN} 1 \mathrm{~A}(\uparrow)$, ODC $(\downarrow), \operatorname{ACTB}(\uparrow)$, CASP4 $(\uparrow)$, OAT $(\downarrow), \operatorname{PMP} 22(\uparrow)$, and ALDH1A1 $(\downarrow)$. EFHU1, ODC, OAT and PMP22 were also modulated in the carrots group and CDKN1A also in the carrots and peas group (see Figure 2). No roles for EFHU1, COX4I1, ACTB and PMP22 in possible protective mechanisms against CRC have previously been reported (see Table 5). Also, except for ACTB, no relation with vegetables or vegetable components has been described. ACTB, although widely used as internal standard for gene expression studies, has previously been shown to be regulated by nutritional interventions, including a vitamin A deficiency study, ${ }^{27.29}$ 
Table 5. Presentation of differentially expressed genes, the direction of the effect in the individual vegetable group compared to control, and the theoretical effect on cancer risk.

\begin{tabular}{|c|c|c|c|c|c|}
\hline $\begin{array}{l}\text { Gene Name by } \\
\text { NCBI } \\
\text { (Abbreviation) }^{1}\end{array}$ & $\begin{array}{c}\text { Cauliflower } \\
\text { group }\end{array}$ & $\begin{array}{l}\text { Carrots } \\
\text { group }\end{array}$ & Peas group & $\begin{array}{l}\text { Onions } \\
\text { group }\end{array}$ & $\begin{array}{c}\text { Theoretical effect } \\
\text { on cancer risk }\end{array}$ \\
\hline HSPD1 & - & - & - & - & - \\
\hline EFHU1 & - & - & & & Unknown \\
\hline COX4II & + & & & & Unknown \\
\hline CDKNIA & + & + & + & & - \\
\hline ODC & - & - & & & - \\
\hline ACTB & + & & & & Unknown \\
\hline CASP4 & + & & & & - \\
\hline OAT & - & - & & & + \\
\hline PMP22 & + & + & & & Unknown \\
\hline ALDHIAI & - & & & & + \\
\hline BNIP1 & & - & & - & Unknown \\
\hline TOP2A & & & & - & - \\
\hline SCD2 & & & & + & Unknown \\
\hline TMSB10 & & & & - & + \\
\hline SEPPI & & & & + & Unknown \\
\hline HIF1A & & & & + & - \\
\hline IL.18 & & & + & & - \\
\hline
\end{tabular}

'NCBI (National Center for Biotechnology Information): hrtp://www.ncbi.nlm.nih.gov/.

CDKN1A, ODC and OAT are involved in cell growth, although via different mechanisms. CDKN1A encodes for a potent cyclin-dependent kinase (CDK) inhibitor, which binds to and inhibits the activity of cyclin-CDK2- or CDK4 complexes, thereby preventing $G_{1}-S^{10}$ and $G_{2}-M^{31}$ transition, which results in cell cycle arrest. The expression of this gene is tightly regulated by the tumour suppressor protein p53. ${ }^{30}$ Next to its ability to block cell cycle progression, CDKN1A mediates apoptosis. ${ }^{32}$ Several vegetable components have been shown to induce CDKN1A expression in titro, like the flavonoids apigenin $^{33}$ and genistein ${ }^{34}$, indole-3- carbinol $^{35}$ and isothiocyanates ${ }^{36}$ present in Brassica vegetables like cauliflower, and beta-carotene ${ }^{37}$ present in carrots. This is the first in min study, in which CDKN1A is reported to be significantly up-regulated by cauliflower, carrots and peas.

Two other genes implicated in cell growth are OAT and ODC. These two genes are involved in polyamine metabolism and were down-regulated in the cauliflower- and carrots group. The protein-product of OAT converses ornithine to glutamate semialdehyde, thereby reducing intracellular ornithine contents. Ornithine is the substrate of ODC, which is the first enzyme in polyamine synthesis. Increases in intracellular polyamine levels are associated with rapid growth, including tumorigenesis. ${ }^{30}$. $\mathrm{ODC}$ is a transcriptional tanget of C-MYC and a modifier of adenomatous polyposis coli (APC)- 
dependent tumorigenesis. Wild-type APC regulates the expression of C-MYC and its antagonist, MAD1, causing an inhibition of ODC expression, a decrease in polyamine metabolism, and reduced tumorigenesis. ${ }^{40}$ Inhibition of ODC $\mathrm{mRNA}$ has been described for $\beta$-carotene ${ }^{41}$ and indole-3-carbonol ${ }^{42}$, present in carrots and cauliflower, respectively, which could explain the reduction in these two diet groups.

CASP4, up-regulated in the cauliflower group, is implicated in apoptosis (programmed cell death). Apoptosis is a general feature in the intestinal crypt, by eliminating differentiated epithelial cells which form the intestinal lining. Programmed cell death occurs also deep in the proliferative zone of the intestinal crypt. Here, genetically damaged stem cells are removed before they can undergo clonal expansion and thereby the occurrence of neoplasia can be prevented.4 Sequential activation of caspases plays a central role in the execution phase of cell apoptosis.4 Several studies have shown an induction of apoptosis by certain flavonoids ${ }^{13},{ }^{45}$, glucosinulate breakdown products ${ }^{46}, 47$ and carotenoids ${ }^{48}-50$, however, no investigation of CASP4 expression was performed.

ALDH1A1 expression was significantly down-regulated in the cauliflower group. It encodes for an enzyme important in ethanol detoxification, metabolism of neurotransmitters, and synthesis of retinoic acid. ${ }^{51,52}$ Ethanol and retinol share chemical similarities and can be oxidized to aldehydes (acetaldehyde and retinal, respectively), and these are oxidized to carboxylic acids (acetate acid and retinoic acid, respectively) by the same enzymes (alcohol dehydrogenase and aldehyde dehydrogenase). ${ }^{52}$ Epidemiological and experimental studies have revealed that acetaldehyde rather than ethanol itself is responsible for the adverse effects of alcohol consumption on CRC. Acetaldehyde is highly toxic, mutagenic and carcinogenic. ${ }^{51}$

Summarizing the effects in the cauliflower group, six out of ten modulated genes could be involved in CRC protective mechanisms; four (HSPD1, CDKN1A, ODC and CASP4) out of these six are affected in such a way that CRC protective mechanisms could be expected mostly related to cell growth regulation and apoptosis.

BNIP1 was down-regulated in the carrots and onions group. No modulation of BNIP1 mRNA or enzyme activity by vegetables or nutrition has been described yet. The protein product of the BNIP1 gene interacts with members of the BCL.2 family, which consists of pro-apoptotic (such as $\mathrm{BAX}$ ) and anti-apoptotic (such as $\mathrm{BCl}$.2) proteins. It is suggested that BNIP1 has pro-apoptotic properties, by interacting with BCL.2 and BCL2L1. However, the precise function of BNIP1 is unclear. 53,54

In total seven genes were affected by carrots. For five of these genes, CRC protective mechanisms are described. For three (HSPD1, CDKN1A and ODC) of these five genes, the direction of the regulation is in favour of CRC protection.

Next to BNIP1 and HSPD1, onions affected the expressions of the genes TOP2A, SCD2, TMSB10, SEPP1 and HIF1A. No roles for SCD2, SEPP1 and HIF1A in possible protective mechanisms against $\mathrm{CRC}$ have been reported and no relation, except 
for HIF1A, with vegetables or vegetable components has been described. HIF1A is a major regulator of the hypoxic response. During hypoxia, HIF1A translocates to the nucleus and facilitates transcription of genes involved in glycolysis and angiogenesis. 55,56 Until recently, hypoxia has been the only naturally occurring signal shown to activate this gene. However, it was shown that the dietary flavonoid quercetin was able to increase HIF1A protein under normal oxygen concentrations. ${ }^{56}$ In the present study, HIF1A was up-regulated in the onions group, which can be caused by flavonoids present in the onions. The possible importance of these genes in CRC and their regulation by vegetables has to be further studied.

TOP2A catalyzes DNA topological reactions via a DNA breakage/reunion mechanism. These DNA topological reactions allow TOP2A to remove excess DNA supercoils, ${ }^{57}$ The breakage/reunion reaction of TOP $2 \mathrm{~A}$ can be interrupted by many anticancer drugs, resulting in the accumulation of a topoisomerase II-DNA covalent intermediate: the cleavage complex. Accumulation of this complex causes tumour cell death. ${ }^{58}$ The inhibition of TOP2A has been shown to be an important therapeutic mechanism in cancer chemotherapy. Nutritional inhibition of the TOP2A enzyme in colon cancer cells has been described for the isoflavone genistein, a major component of soy. ${ }^{5}$ ?

TMSB10 encodes for a $\mathrm{G}$-actin binding protein which has dual functions: induction of programmed cell death and cell growth and -differentiation. ${ }^{60.62}$ TMSB10 was significantly down-regulated in the onions group compared to the control group. Retinoic acid is known to increase TMSB10 mRNA and apoptosis in cancer cells. ${ }^{63}$ However, in the present study, no difference in TMSB10 expression was found in the carrots group compared to the control group, suggesting that the amount of possible bioactive compounds like carotenoids and retinoids are too low to exert an effect on TMSB10 expression.

Reviewing the results on gene expression in the onions group, four out of seven genes were modulated which could be involved in CRC protective mechanisms. For three (HSPD1, TOP2A and TMSB10) of these four genes the expression was affected in such a way that CRC protective mechanisms could be stimulated by maintaining homeostasis and DNA stability, and by reducing cell growth and induction of cell differentiation.

IL18 was significantly up-regulated in the peas group compared to the control and carrots group. Expression in the cauliflower and onions group was lower, although not significantly. IL 18 encodes for a type-1 T-helper cytokine which has antitumour activities by increasing apoptosis ${ }^{64}, 65$, inhibiting angiogenesis ${ }^{66}$ and maintaining homeostasis. ${ }^{67}$ Little information is available about nutritional modulation of IL18. In two studies, a reduction in serum IL-18 was observed after a high fibre diet. 08,69

Least modulations took place in the peas group, however, the modulation of these three genes (HSPD1, IL18 and CDKN1A) are expected to increase CRC protective mechanisms. 
Next to this evaluation of the specific gene effects of the individual vegetables and their possible role in CRC prevention, it is interesting to compare the results of the current individual vegetable study with the previous performed vegetable mixture study described in Chapter 3. In the Venn diagrams (Figure 3A-D), the corresponding and differing genes between each vegetable group and the $40 \%$ vegetable mixture group are shown.

Eleven genes were significantly modulated in both studies. For seven of these eleven genes, the result in the vegetable mixture study can be expected from the results of the individual vegetables on the expression of these genes. The results of the present study reveal which vegetables were (mainly) accountable for these same effects. ACTB was modulated by the cauliflowers; OAT, EFHU1 and PMP22 were modulated by the cauliflowers and carrots; and, SCD2 and HIF1A were modulated by the onions; in the same direction as observed in the vegetable mixture study. CASP4 was significantly increased in the $40 \%$ vegetable mixture group as compared to the $10 \%$ and $20 \%$ vegetable mixtures group. In the present study, this gene was only significantly up-regulated in the cauliflower group. For three genes, i.e. ALDH1A1, TMSB10 and SEPP1, the effect of the vegetable mixture resulted in an opposite outcome as would be expected by the effects of the individual vegetables.

Seven genes which were modulated in the present study on individual vegetables were not modulated in the study on mixed vegetables. An explanation could be that the bioactive compounds present in the individual vegetables possibly neutralize each other in the mixture. Fourteen genes which were significantly affected in the $40 \%$ group of vegetable mixture study, were not significantly modulated in at least one of the individual vegetable groups of the present study, It is suggested that the individual vegetables contained not enough bioactive compounds to exert a measurable effect on gene expression. The combined effect could be additive or synergistic and therefore large enough to cause a gene expression effect.

Almost half of the affected genes which were modulated are currently not known to be involved in CRC protective mechanisms or known to be modulated by vegetables. Their possible role in CRC prevention and their modulation by vegetables are not clear and have to be further investigated. However, a number of genes was modulated in such a way CRC preventive mechanisms could be activated and this already occurred through diets relatively low in vegetable amount. The four individual vegetables seem to have about the same potential of modulating genes in favour of lower CRC risk, although via different mechanisms. Furthermore, a number of similar genes were modulated by the respective vegetables individually as well as mixed. These similar modulated genes are of particular interest because they are modulated in both studies and could play an important role in CRC prevention by vegetables. 


\section{References}

1. World Heaith Organization \& International Agency for Research on Cancer. World Cancer Report. Lyon: IARCPress, 2003.

2. Potter JD, Colorectal cancer: molecules and populations. J Natl Cancer Inst 1999;91:916-32.

3. World Cancer Research Fund. Food, Nutrition and the Prevention of Cancer, a global perspective. Washington: BANTA Book group, 1997.

4. World Health Organization. The World Health Report 1997: Conquering suffering Enriching humanity. Geneva: WHO, 1997.

5. Steinmetz KA, Potter JD, Vegetables, fruit, and cancer prevention: a review. J Am Diet Assoc 1996;96:1027-39.

6. Steinmetz KA, Potter JD. Vegetables, fruit, and cancer. I. Epidemiology. Cancer Causes Control 1991;2:325-57,

7. Steinmetz KA, Potter JD. Vegetables, fruit, and cancer. II. Mechanisms. Cancer Causes Control $1991: 2: 427-42$

8. Murillo G, Mehta RG. Cruciferous vegetables and cancer prevention. Nutr Cancer 2001;41:17-28.

9. van Poppel G, Verhoeven DT, Verhagen H, Goldbohm RA. Brassica vegetables and cancer prevention. Epidemiology and mechanisms. Adv Exp Med Biol 1999;472:159-68.

10. Guyonnet D, Siess MH, Le Bon AM, Suschetet M. Modulation of phase II enzymes by organosulfur compounds from allium vegetables in rat cissucs. Toxicol Appl Pharmacol 1999,154:50-8.

11. Bianchini $\mathrm{F}$, Vainio $\mathrm{H}$. Allium vegetables and organosulfur compounds: do they help prevent cancer? Environ Health Perspect 2001;109:893-902.

12. van Kranen HJ, van lersel PW, Rijnkels JM, Beems DB, Alink GM, van Kreijl CF, Effects of dietary fat and a vegetable-fruit mixture on the development of intestinal neoplasia in the ApcMin mouse. Carcinogenesis 1998;19:1597-601.

13. Rijnkels JM, Alink GM. Effects of a vegetables-fruit mixture on liver and colonic 1,2dimethylhydrazine-metabolizing enzyme activities in rats fed low- or high-fat diets. Cancer Lett 1998:128:171-5.

14. Rijken PJ, Timmer WG, van de Kooij AJ, van Benschop IM, Wiseman SA, Meijers M, Tijburg L.B. Effect of vegetable and carotenoid consumption on aberrant crypt multiplicity, a surrogate end-point marker for colorectal cancer in azoxymethane-induced rats. Carcinogenesis 1999;20:2267-72.

15. Schatzkin A, Lanza E, Corle D, Lance P, Tber F, Caan B, Shike M, Weissfeld J, Burt R, Cooper MR, Kikendall JW, Cahill J. Lack of effect of a low-fat, high-fiber diet on the recurrence of colorectal adenomas. Polyp Prevention Trial Study Group [see comments]. N Engl J Med 2000-342:1149-55.

16. Gamet-Payrastre I, Li P, Lumeau S, Cassar G, Dupont MA, Chevolleau S, Gase N, Tulliez J, Terce F. Sulforaphane, a naturally occurring isothocyanate, induces cell cycle arrest and apoptosis in HT29 human colon cancer cells. Cancer Res 2000;60;1426-33.

17. Mahmoud NN, Carothers AM, Grunberger D, Bilinski RT, Churchill MR, Martucci C, Newmark $\mathrm{HL}_{2}$ Bertagnolli MM. Plant phenolics decrease intestinal tumors in an animal model of familial adenomatous polyposis. Careinogenesis 2000;21:921-7.

18. Wenzel U, Kuntz S, Brendel MD, Daniel H. Dietary flavone is a potent apoptosis inducer in human colon carcinoma cells. Cancer Res 2000;60-3823-31.

19. Hasseman J. Chen E, Yang I, Quackenbush J, Aminoallyl labeling of RNA for microarrays. http://www.tigr.org/tdb/micoarray/protocolsTIGR.shtml (Accessed June 21, 2001), 2001. 
20. Ketr MK. Design considerations for efficient and effective microatray studies. Biometrics 2003:59:822-828.

21. Kerr MK, Martin M, Churchill GA. Analysis of variance for gene expression microarray data. Joumal of Computational Biology 2000;7:819-837. Assessing gene significance from cDNA microarray expression data via mixed models. J Comput Biol 2001;8:625-37.

23. Neter J, Kutner MH, Nachtsheim C], Wasserman W. Applied Linear Statistical Models (Fourth Edition), Mass, 1996.

24. Kerr MK, Afshan CA, Bennett L, Bushel P, Martinez J. N.J. W, Churchill GA. Statistical analysis of a gene cxpression microarray experiment with replication. Statistica Sinica 2002;12:203-217.

25. Garrido C, Gurbuxani S, Ravagnan L. Kroemer G. Heat shock proteins: endogenous modulaton of apoptotic cell death. Biochem Biophys Res Commun 2001;286:433-42.

26. Jindal S. Heat shock proteins: applications in health and disease. Trends Biotechnol 1996;14:17-20.

27. Yamada H, Chen D, Monstein HJ, Hakanson R. Effects of fasting on the expression of gastrin, cholecystokinin, and somatostatin genes and of various housekeeping genes in the pancreas and upper digestive tract of rats. Biochem Biophys Res Commun 1997;231:835-8.

28. Selvey S, Thompson EW, Matthaei K, Lea RA, Irving MG, Griffiths LR. Beta-actin-an unsuitable internal control for RT-PCR. Mol Cell Probes 2001:15:307-11.

29. Nur T, Peijnenburg AA, Noteborn HP, Baykus H, Reifen R. DNA microarray technology reveals similar gene expression patterns in rats with vitamin a deficiency and chemically induced colitis. I Nutr 2002;132:2131-6.

30. el-Deiry WS, Tokino T, Velculescu VE, Levy DB, Parsons R, Trent JM, Lin D, Mercer WE, Kinzler KW, Vogelstein B. WAF1, a potential mediator of p53 tumor suppression. Cell 1993;75:817-25.

31. Bunz F, Dutriaux A, Lengauer C, Waldman T, Zhou S, Brown JP, Sedivy JM, Kinzler KW, Vogelstein B. Requirement for $\mathrm{p} 53$ and $\mathrm{p} 21$ to sustain G2 arrest after DNA damage. Science $1998 ; 282: 1497-501$.

32. Levkau B, Koyama H, Raines EW, Clurman BE, Herren B, Orth K, Roberts JM, Ross R. Cleavage of $21 \mathrm{Cip} 1 / \mathrm{Wafl}$ and $\mathrm{p} 27 \mathrm{Kip} 1$ mediates apoptosis in endothelial cells through activation of Cdk2: role of a caspase cascade. Mol Cell 1998;1:553-63,

33. Shukla S, Gupta S. Molecular mechanisms for apigenin-induced cell-cycle arrest and apoptosis of hormone refractory human prostate carcinoma DU145 cells. Mol Carcinog 2004;39:114-26.

34. Kobayashi T, Nakata T, Kuzumaki T. Effect of flavonoids on cell cycle progression in prostate cancer cells. Cancer Lett 2002;176:17-23.

35. Firestone GL, Bjeldanes LF. Indole-3-carbinol and 3-3'-dindolylmethane antiproliferative signaling pathways control cell-cycle gene transcription in human breast cancer cells by regulating promoterSpl transcription factor interactions. I Nutr 2003;133:2448S-2455S.

36. Chiao JW, Chung F, Krzeminski J, Amin S, Arshad R, Ahmed T, Conaway CC. Modulation of growth of human prostate cancer cells by the $\mathrm{N}$-acerylcysteine conjugate of phenethyl isothiocyanate. Int J Oncol 2000;16:1215-9.

37. Palozza P, Serini S, Torsello A, Boninsegna A, Covaca V, Maggiano N, Ranelletti FO, Wolf FI, Calviello G. Regulation of cell cycle progression and apoptosis by beta-carotene in undifferentiated and differentiated HL-60 leukemia cells: possible involvement of a redox mechanism. Int J Cancer 2002;97:593-600. 
38. Seiler N, Omithine aminotransferase as a therapeutic target in hyperammonemias. Adv Exp Med Biol 1997;420:113-42.

39. Han X, Kazarinoff MN, Seiler N, Stanley BA. Rat colon ornithine and arginine metabolism: coordinated effects after proliferative stimuli. Am J Physiol Gastrointest Liver Physiol 2001:280:G389-99.

40. Martincz ME, O'Brien TG, Fultz KE, Babbar N, Yerushalmi H, Qu N, Guo Y, Boorman D, Einspahr J, Alberts DS, Gemer EW. Pronounced reduction in adenoma recurrence associated with aspirin use and a polymorphism in the ornithine decarboxylase gene. Proc Natl Acad Sci U S A 2003;100:7859-64.

41. Phillips RW, Kikendall JW, Luk GD, Willis SM, Murphy JR, Maydonovitch C, Bowen PE, Stacewicz-Sapuntzakis M, Wong RK. beta-Carotene inhibits rectal mucosal omithine decarboxylase activity in colon cancer patients. Cancer Res 1993;53:3723-5.

42. Shertzer HG, Senft AP. The micronutrient indole-3-carbinol: implications for disease and chemoprevention. Drug Metabol Drug Interact 2000;17:159-88.

43. Johnson IT. Anticarcinogenic effects of diet-related apoptosis in the colorectal mucosa. Food Chem Toxicol 2002;40:1171-8.

44. Kamada S, Washida M, Hasegawa J, Kusano H, Funahashi Y, Tsujimoto Y. Involvement of caspase4(-like) protease in Fas-mediated apoptotic pathway. Oncogene 1997;15:285-90.

45. Mertens-Taleot SU, Talcott ST, Petcival SS. Low concentrations of quercetin and ellagic acid synergistically influence proliferation, cytotoxicity and apoptosis in MOLT-4 human leukemia cells. J Nutr 2003:133:2669-74.

46. Gingras D, Gendron M, Boivin D, Moghrabi A, Theoret Y, Beliveau R. Induction of medulloblastoma cell apoptosis by sulforaphane, a dietary anticarcinogen from Brassica vegetables. Cancer Lett 2004:203:35-43.

47. Hu R, Kim BR, Chen C, Hebbar V, Kong AN. The roles of JNK and apoptotic signaling pathways in PEITC-mediated responses in human HT-29 colon adenocarcinoma cells. Carcinogenesis $2003 ; 24: 1361-7$.

48. Palozza P, Calviello G, Serini S, Maggiano N, Lanza P, Ranelletti FO, Bartoli GM. beta-carotene at high concentrations induces apoptosis by enhancing oxy-radical production in human adenocarcinoma cells. Free Radic Biol Med 2001;30:1000-7.

49. Palozza P, Maggiano N, Calviello G, Lanza P, Piccioni E, Ranelletti FO, Bartoli GM. Canthaxanthin induces apoptosis in human cancer cell lines. Carcinogenesis 1998;19:373-6.

50. Palozza P, Serini S, Maggiano N, Angelini M, Boninsegna A, Di Nicuolo F, Ranelletti FO, Calviello G. Induction of cell cycle arrest and apoptosis in human colon adenocarcinoma cell lines by betacarotene through down-regulation of cyclin $\mathrm{A}$ and $\mathrm{Bcl}-2$ family proteins. Carcinogenesis 2002;23:118.

51. Seitz HK, Natsuzaki S, Yokoyama A, Homann N, Vakevainen S, Wang XD. Alcohol and cancer. Alcohol Clin Exp Res 2001;25:137S-143S.

52. Crabb DW, Pinairs J. Hasanadka R, Fang M, Leo MA, Lieber CS, Tsukamoto H, Motomura K. Myyahara T, Ohata M, Bosron W, Sanghani S, Kedishvil N, Shiraishi H, Yokoyama H, Mryagi M, Ishii H, Bergheim I, Menzl I, Parlesak A, Bode C. Alcohol and retinoids. Alcohol Clin Exp Res 2001:25:207S-217S.

53. Zhang H, Heim J. Meyhack B. Novel BNIP1 variants and their interaction with BCl.2 family members. FEBS 1 ett 1999;448:23-7. 
54. Yasuda M, Chinnadurai G. Functional identification of the apoptosis effector BH3 domain in cellulat protein BNIP1. Oncogene 2000:19:2363-7.

55. Goda N, Ryan HE, Khadivi B, MeNulty W, Rickert RC, Johnson RS. Hypoxia-inducible factot 1alpha is essential for cell cycle arrest during hypoxia. Mol Cell Biol 2003;23:359-69.

56. Wilson WJ. Poellinger L. The dietary flavonoid quereetin modulates HIF-1 alpha activity in endothelial cells. Biochem Biophys Res Commun 2002;293:446-50.

57. Watt PM, Hickson ID. Structure and function of type II DNA topoisomerases. Biochem J 1994;303 (Pt 3):681-95.

58. Liu LF. DNA topoisomerase poisons as antitumor drugs. Annu Rev Biochem 1989;58:351-75.

59. Salti GI, Grewal S, Mehta RR, Das Gupta TK, Boddie AW, Jr., Constantinou At. Genistein induces apoptosis and topoisomerase II-mediated DNA breakage in colon cancer cells. Eur J Cancer 2000,36:796-802

60. Lee SH, Zhang W, Choi J, Cho YS, Oh SH, Kim JW, Hu L, Xu J, Lu J, Lee JH. Overexpression of the thymosin beta-10 gene in human ovarian cancer cells disrupts $\mathrm{F}$-actin stress fiber and leads to apoptosis. Oncogene 2001;20:6700-6.

61. Guticrex-Pabello JA, McMurray DN, Adarns LG. Upregulation of thymosin beta-10 by Mycobacterium bovis infection of bovine macrophages is associated with apoptosis. Infect Immun 2002;70:2121-7.

62. Santelli G, Califano D, Chiappetta G, Vento MT, Bartoli PC, Zullo F, Trapasso F, Viglietto G, Fusco A. Thymosin beta-10 gene overexpression is a general event in human carcinogenesis. Am J Pathol 1999:155:799-804.

63. Hall $\mathrm{AK}$, Liarozole amplifies retinoid-induced apoptosis in human prostate cancer cells. Anticancer Drugs 1996; 7:312-20.

64. Dinarello CA. Interleukin-18. Methods 1999;19:121-32,

65. Marino E, Cardier JE. Differential effect of IL-18 on endothelial cell apoptosis mediated by TNFalpha and Fas (CD95). Cytokine 2003;22:142-8.

66. Coughlin CM, Salhany KE, Wysocka M, Aruga E, Kurzawa H, Chang AE, Hunter CA, Fox JC, Trinchieri G, Lee WM. Interleukin-12 and interleukin-18 synergistically induce murine tumor regression which involves inhibition of angiogenesis. J Clin Invest 1998;101:1441-52.

67. Pages F, Berger A, Henglein B, Piqueras B, Danel C, Zinzindohoue F, Thiounn N, Cugnene PH,

- Fridman WH. Modulation of interleukin-18 expression in human colon carcinoma: consequences for tumor immune surveillance. Int J Cancer 1999;84:326-30.

68. Esposito K, Pontillo A, Di Palo C, Giugliano G, Masella M, Marfella R, Giugliano D. Effect of weight loss and lifestyle changes on vascular inflammatory markers in obese women: a randomized trial. Jama 2003;289:1799-804.

69. Esposito K, Nappo F, Giugliano F, Di Palo C, Ciotola M, Barbieri M, Paolisso G, Giugliano D. Meal modulation of circulating interleukin 18 and adiponectin concentrations in healthy subjects and in patients with type 2 diabetes mellitus. Am J Clin Nutr 2003;78:1135-40. 



\section{Chapter 6}

\section{Gene expression modulation in lung cancer preventive pathways by vegetables in female C57BL6 mice}

In preperation

S.G.J. van Breda'

E. van Agen'

S. van Sanden ${ }^{2}$

T. Burzykowski²

J.C.S. Kleinjans ${ }^{1}$

J.H.M. van Delft'

'Department of Health Risk Analysis and Toxicology, Maastricht University, Maastricht, The Netherlands 2Center for Statistics, Limburg University Centre, Diepenbeek, Belgium 


\section{Abstract}

Worldwide, lung cancer is the most prevalent and lethal malignant disease. In addition to avoidance of the most predominant risk factor, i.e. tobacco use, consumption of high amounts of vegetable and fruits is the most effective means of preventing lung cancer. However, the molecular mechanisms underlying lung cancer risk prevention by vegetables are not clear. In the present study, the effect of vegetables on gene expression changes in the lungs of female C57BL6 mice was investigated using cDNA microarray technology. Therefore, mice were fed one of eight different diets for a period of two weeks: 1) a diet containing no vegetables; a diet containing a vegetable mixture: 2) $10 \% \mathrm{wt} / \mathrm{wt}$; 3) $20 \%$ wt $/ w$; or 4) $40 \% \mathrm{wt} / \mathrm{wt}$; or a diet containing individual vegetables: 5) cauliflower; 6) carrots; 7) peas; or 8 ) onions. The vegetables mixture consisted of these four individual vegetables. After sacrifice, the lungs were removed and total RNA was isolated from the lungs for expression analysis of 602 genes. The results of this study suggest that individual vegetables have a higher potential of modulating genes ( 5 out of 8 ) in favor of lung cancer risk prevention, in stead of the vegetable mixture ( 2 out of 7 ); the other gene modulations are expected to enhance lung cancer risk. The involved pathways are various and include cell growth, apoptosis, biotransformation and immune response. Furthermore, carrots were able to modulate most gene expressions, and most of these effects also favor lung cancer risk prevention. The current study provides more insight into the genetic mechanisms by which vegetables, in particular carrots, can prevent lung cancer risk. 


\section{Introduction}

Lung cancer is the most prevalent and lethal malignant disease in the world, accounting for almost 16 percent of al new cancer cases. Approximately $90 \%$ of the people who develop lung cancer will die from it. No effective treatment is available; the five years survival nate for lung cancer patients is less than 15 percent. Thus the need for primary prevention of lung cancer is paramount.

The most effective means of preventing lung cancer is avoidance of tobacco use. Cigarette smoking accounts for about 90 percent of cases in men and about 80 percent of cases in women, with the rest due to occupational exposures including asbestos, arsenic, chloromethyl ethers, chromium- $\mathrm{VI}$, and nickel; residential and occupational exposures to radon; and probably exposures to carcinogenic air pollutants in the general environment. ${ }^{2}$ Next to avoiding or reducing these known causative exposures, consumption of diets high in vegetables and fruit is the most effective means for prevention of lung cancer. ${ }^{3}$ The most available and consistent evidence from prospective epidemiological studies is that particularly dark green and yellow-orange vegetables, rich in $\beta$-carotene and vitamin E, are the specific types of vegetables, which best protect against lung cancer. Limited evidence is available regarding the preventive potential of legumes like beans and peas against lung cancer. $^{3}$

A lot of the research on vegetables and lung cancer has been motivated by the hypothesis that these vegetables contain relatively high amounts of specific micronutrients with specific anticarcinogenic potential. Observational epidemiologic studies have demonstrated a statistically significant decreased relative risk of lung cancer between the extreme quintiles or quartiles of vegetable intake and serum levels of these agents. 4,5 These agents would have antioxidative capacities thereby protecting against oxidative DNA damage resulting in lower cancer risk. ${ }^{5.9}$ However, the results of the randomised controlled trials in which the effect of daily high doses of $\beta$-carotene, vitamin $A$ and/or vitamin $E$ administrated orally during several years on lung cancer incidence was investigated, were disappointing. ${ }^{10-13}$ Instead of a protective effect, adverse effects on lung cancer development were reported. It has been suggested that higher concentrations of these antioxidants resulting from the supplementations have pro-oxidant effects, inducing DNA damage and membrane instability. Furthermore, the results of these trials also emphasize that it is not known whether the epidemiological associations are specific for the micronutrients or whether the micronutrient measurements are merely serving as a marker of the intake of other protective substances or even more healthful dietary habits in general. In addition, the complex mixture of numerous substances rather than a single constituent in vegetables could be responsible for the net effect. 
The vegetable components that reach the lung may have been modulated by the first-pass metabolism: substances that are absorbed via intestinal blood capillaries will first have to pass the liver before they reach the systemic circulation. Here, many substances are extensively metabolized. Therefore, the lungs are regarded as a systemic organ to study the effect of vegetables, because they are subject to only systemic exposure of vegetablederived components. The mechanisms by which vegetables protect against lung cancer could therefore be different compared to for instance the colon and rectum. The gastrointestinal tract, including the colon and rectum, is side of contact, i.e., as the vegetables pass through the tract, they come into close contact with the intestinal lining.

The specific mechanisms by which vegetables exert lung cancer risk prevention are unknown and need to be elucidated. The number of studies in which the effect of whole vegetables instead of a specific constituent has been examined is limited. Changes in the multi-gene expression patterns can provide clues about regulatory mechanisms and broader cellular functions and biochemical pathways. Current DNA microarray technology allows the simultaneous expression analysis of a large numbers of genes. ${ }^{14}$

Therefore, in the present study, microarray technology was used to gain more information about the effect of vegetables in the lungs at the level of the expression for multiple genes involved in various genetic pathways underlying cancer risk prevention. Two different approaches were taken: in the first approach, the dose-dependent effect of a mixture of four vegetables (cauliflower, carrots, peas and onions) on gene expression changes in the lung was examined; in the second approach, the contribution of the individual vegetables present in the vegetable mixture, was investigated.

\section{Materials and Methods}

\section{Animals and Diet}

The number and type of animals, the development of the vegetable diets and the treatment of the animals in the present study was previously described in Chapter $\mathbf{3}$ and $\mathbf{5}$ (Section Materials and Methods; Animals and Diet). In short, eight-week-old female C57BL6 mice (Charles River Laboratories, Lyon, France) were randomly assigned to one of eight different diets for a period of two weeks: 1) a diet containing no vegetables (control diet; two groups of six and seven animals received the control diet, referred to as control group $\mathrm{I}$ and II); a diet containing a vegetable mixture: 2) $10 \%$ wt/wt vegetables mixture; 3) $20 \%$ wt/wt vegetables mixture; or 4) $40 \% \mathrm{wt} / \mathrm{wt}$ vegetables mixture; or a diet containing individual vegetables: 5) cauliflower; 6) carrots; 7 ) peas; or 8 ) onions (six or seven animals per group). The vegetables mixture used consisted of the four individual vegetables used in diet $5-8$, i.e. cauliflower ( $30 \%$ wet wt), carrots $(30 \%$ wet wt), peas $(30 \%$ wet wt), and onions ( $10 \%$ wet wt). 
Tabke 1. Composition of the eighe diets in $\mathrm{g} / \mathrm{kg}$.

\begin{tabular}{|c|c|c|c|c|c|c|c|c|}
\hline Ingredients & $\begin{array}{c}\text { Control } \\
\text { diet }^{1} \\
\end{array}$ & $\begin{array}{c}10 \% \text { Vegetable } \\
\text { diet }^{2} \\
\end{array}$ & $\begin{array}{c}20 \% \text { Vegetable } \\
\text { diet }^{2} \\
\end{array}$ & $\begin{array}{c}40 \% \text { Vegetable } \\
\text { diet }^{2} \\
\end{array}$ & $\begin{array}{c}\text { Caulislower } \\
\text { diet }^{2}\end{array}$ & $\begin{array}{l}\text { Carrots } \\
\text { diei }^{I}\end{array}$ & $\begin{array}{l}\text { Peas } \\
\text { dict }^{2}\end{array}$ & $\begin{array}{c}\text { Onions } \\
\text { diet }^{2}\end{array}$ \\
\hline Cerelose/Dextrose & 515 & 422 & 329 & 143 & 451 & 452 & 303 & 488 \\
\hline DI-Methionine & 2 & 2 & 2 & 2 & 2 & 2 & 2 & 2 \\
\hline $\mathrm{NaCl}$ & 3 & 3 & 3 & 3 & 3 & 3 & 3 & 3 \\
\hline Choline CL. $50 \%$ & 4 & 4 & 4 & 4 & 4 & 4 & 4 & 4 \\
\hline Soya oil & 50 & 50 & 50 & 50 & 50 & 50 & 50 & 50 \\
\hline $\mathrm{KCl}$ & 7 & 7 & 7 & 7 & 7 & 7 & 7. & 7 \\
\hline $\mathrm{KH}_{2} \mathrm{PO}_{4}$ & 7 & 7 & 7 & 7 & 7. & 7 & 7 & 7 \\
\hline $\mathrm{MgO}$ & 2 & 2 & 2 & 2 & 2 & 2 & 2 & 2 \\
\hline $\mathrm{CaHPO}_{4} 2 \mathrm{H}_{2} \mathrm{O}$ & 13 & 13 & 13 & 13 & 13 & 13 & 13 & 13 \\
\hline $\mathrm{CaCO}_{3}$ & 10 & 10 & 10 & 10 & 10 & 10 & 10 & 10 \\
\hline Corn Starch. & 100 & 100 & 100 & 100 & 100 & 100 & 100 & to0 \\
\hline Dicacel/Cellulose & 77 & 70 & 63 & 49 & 71 & 67 & 63 & 73 \\
\hline Casein & 200 & 200 & 200 & 200 & 2000 & 200 & 200 & 200 \\
\hline $\mathrm{MgSO}_{4} 7 \mathrm{H}_{2} \mathrm{O}$ & 4 & 4 & 4 & 4 & 4 & 4 & 4 & 4 \\
\hline $\begin{array}{l}\text { Standard vitamun } \\
\text { premix }\end{array}$ & 3 & 3 & 3 & 3 & 3 & 3 & 3 & 3 \\
\hline $\begin{array}{l}\text { Standard } \\
\text { mecronutrient premix }\end{array}$ & 3 & 3 & 3 & 3 & 3 & 3 & 3 & 3 \\
\hline Cauliflower $^{3}$ & 0 & 17.5 & 35 & 70 & 70 & & & \\
\hline Carrots" & 0 & 18.3 & 36.5 & 73 & & 73 & & \\
\hline Peas ${ }^{3}$ & 0 & 56.5 & 113 & 226 & & & 226 & \\
\hline Onons $^{3}$ & 0 & 7.7 & 15.5 & $3 !$ & & & & 31 \\
\hline
\end{tabular}

TControl diet is the basal $20 \%$. casen reference dict.

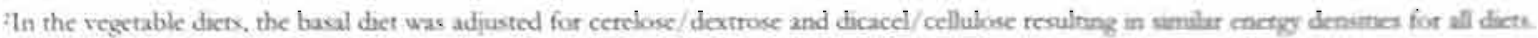

Wet weight: cauliflower $1.8 \mathrm{~kg}$; carroos $1.8 \mathrm{~kg}$; peas $1.8 \mathrm{~kg}$, onions $0.6 \mathrm{~kg}$ 
The diet in the highest vegetable mixture group contained $40 \%$ of this vegetable mixture. The amount of vegetables used in the individual vegetable diets is equal to the amount of these specific vegetables in the $40 \%$ vegetable mixture group. The composition of the different diets is presented in Table 1.

The animals were maintained in the laboratory animal care facilities at Maastricht University under controlled environmental conditions (temperature $21 \pm 1{ }^{\circ} \mathrm{C}$, relative humidity $50 \% \pm 10,12$-hr light/dark cycle). Body weights of the mice were recorded weekly. The study was approved by the Institutional Committee of Animal Experimentation Maastricht University.

\section{Tissue Sampling}

Mice were sacrificed by bleeding the vena cava inferior under Nembutal (Sanofi Sante, Maasluis, the Netherlands) anesthesia. Nembutal was administered subcutaneously in the neck at a dose of $60 \mathrm{mg} / \mathrm{kg}$ body weight. The lungs were removed and quickly washed in ice-cold $1 \mathrm{x}$ PBS, immediately frozen in liquid nitrogen and stored at $-80^{\circ} \mathrm{C}$ until use. For total RNA isolation, frozen lung tissue was ground to a powder in a stainless steel mortar under liquid nitrogen and homogenized in $800 \mu \mathrm{l}$ TRIZOL Reagent (Gibco/BRL).

\section{Total RNA Isolation and cDNA Probe Symthesis}

Total RNA was extracted according to the manufacturer's instructions. The RNeasy ${ }^{\circledR}$ Mini Kit (Qiagen, Westburg, Leusden, The Netherlands) together with a DNase treatment was used to purify total RNA from salts and residual DNA. Quantity of each RNA sample was measured by a spectrophotometer and varied from 10 to $30 \mu \mathrm{g}$ per mouse. RNA integrity was determined by a Bioanalyzer (Agilent Technologies Netherlands B.V., Amsterdam, The Netherlands). All samples contained intact total RNA with an rRNA ratio (28S/18S) $>1.5$.

Three RNA pools per diet group were prepared by pooling equal amounts of total RNA from two or three mice. Cyanine 3- (Cy3) and Cyanine 5- (Cy5) labeled cDNA probes were prepared using $3 \mu \mathrm{g}$ total RNA from each pool, by the method of Hasseman et al. ${ }^{15}$

\section{DNA Mianamay Proparation}

This study is part of a project that investigates the effects of vegetables on gene expression in colon mucosa of humans and mice. For the human study, the expression levels of genes in colon mucosa were measured using the PHASE-1 Microarray Human-600 (PHASE-1 Molecular Toxicology, Santa fee, USA). A mouse cDNA microarray was constructed based on the genes present on the PHASE-1 Microarray Human-600. These genes represent a dedicated selection of biologically relevant gene sequences involved in inflammation, DNA 
damage and repair, oxidative stress, cell signaling, cell proliferation, metabolism, transcription and apoptosis.

Selection of mouse cDNA clones, amplification of cDNA inserts by PCR, verification and purification of PCR products and spotting of the microarrays was carried out as described in Chapter 3 (Section Materials and Methods; cDNA Microarnay Preparation).

\section{CDNA Micnamy Hybridizations}

For the hybridizations, within each diet group three pools were formed, each consisting of equal amounts of total RNA of two or three mice. By creating three pools per diet group instead of one, biological variability can be taken into account. This allows for a broader generalizability of the results.

For each of the three sets of pools from the different vegetable mixture groups, a loop design was constructed with four microarrays, as follows: $\mathrm{D} 0 \mathrm{j} \rightarrow \mathrm{Dlj} \rightarrow \mathrm{D} 2 \mathrm{j} \rightarrow \mathrm{D} 3 \mathrm{j} \rightarrow \mathrm{D} 0 \mathrm{j}$ (the pools for control $\mathrm{I}, 10 \%, 20 \%$ and $40 \%$ diet denoted, respectively, by D0j, D1j, D2 $\mathrm{j}$ and $\mathrm{D} 3 \mathrm{j}$ with $\mathrm{j}=1,2$ or 3 ; arrows join the samples put on the same array and indicate the sample labeled with Cy5). The loop is repeated three times, and then analyzed together, to reflect the possible variability in the experimental data. The statistical power of this design for estimating the dose-response profile is higher compared to the classical reference design in which each diet group is compared with the control group. Furthermore, fewer arrays are needed by using this design. ${ }^{16,17}$ In total 12 arrays were used. Cy3 and Cy 5 labeled cDNA probes of two groups were mixed according to this design and hybridized to the cDNA microarray by the method of Hasseman et al. ${ }^{15}$

For each of the three sets of pools from the individual vegetable groups, a reference hybridization design was constructed. ${ }^{17}$ In this design, each vegetable group is compared with the same pool from control group II. Cy3 and Cy5 labeled cDNA probes of the vegetable and control group II were mixed and hybridized to the CDNA microarray by the method of Hasseman et al. ${ }^{15}$ The reference design was chosen because it allows for the same precision of all comparisons of vegetable groups against the control. In order to remove potential bias to dye effects, a second microarray experiment was carried out for each couple, in which the dyes were switched (flip-dye experiment). In total, $24 \mathrm{cDNA}$ microarray hybridizations were performed.

Slides were scanned on a GMS 418 Array Scanner (Affymetrix, High Wycombe, UK). The images obtained (resolution 10 micron; 16 bit tiff image) were processed with ImaGene 5.0 software (Biodiscovery, Los Angeles, USA) to measure mean signal intensities for spots and local background. 


\section{cDNA Microarma Data Processing and Statistical Analysis}

The data were analyzed using ANOVA models ${ }^{16}$, without background correction and using base-2 logarithmic transformation of the measured intensities. Following the suggestions of Kerr et al. (2000), no background-signal correction was applied, as it appeared to add extra unnecessary variability to the data. In the analysis, the base- 2 logarithmically transformed data were used. Due to computational limitations, the models were fit in two stages. The ANOVA model included a normalization step, taking into account both the global (across-genes) and local (gene-specific) normalization. ${ }^{19}$

To investigate dose-dependency, all pairwise differences between the vegetable mixture groups were examined. To examine the contribution of the individual vegetable on gene expression, all pairwise differences between the individual vegetable groups were examined. For each gene, the Tukey procedure was used to correct for multiple comparisons. ${ }^{20}$ To control the overall (across genes) probability of false positive findings, at around $5 \%$, a p-value $<0.0001$ was considered to indicate statistical significance of an individual pairwise comparison.

\section{Real-time RT.PCR}

To verify the cDNA microarray results, 10 gene expression differences, representing five genes which were responsive to vegetables, were analyzed by real-time RT-PCR, as described previously in Chapter 3 (Section Materials and Methods; Real-time RT-PCR). A list of validated genes and the normalization gene and their chosen primers is given in Table 2.

\section{Additional Statistical Anatysis}

Statistical analysis of bodyweight of the mice was carried out using SPSS version 6.1 .1 for Macintosh. Data were analyzed by means of analysis of variance followed by Student's ttest. $A$ two-sided p-value $<0.05$ was considered to indicate statistical significance.

\section{Results}

Mean body weights per week per group are presented in Table 3. During the week prior to the start of the intervention (week 0), all animals received the control diet. At the end of this week, there were no statistical significant differences in mean body weights between the groups. After the intervention, mean body weights were significantly increased within each group $(\mathrm{P}<0.05)$, but no significant differences between the groups were observed. 
Tuble 2. Primer sequences of the validated genes and the 185 normalization gene.

\begin{tabular}{|c|c|c|c|}
\hline $\mathrm{AC} \# 1$ & $\begin{array}{l}\text { Gene Name by NCBI } \\
\text { (Abbreviation) }\end{array}$ & Forward Primes & Reverse Primer \\
\hline BG076021 & $\begin{array}{l}\text { Heat shock } 70 \mathrm{kD} \text { protein } 5 \text { (glucose- } \\
\text { regulated protein) (HSPA5) }\end{array}$ & 5'GAAGGTTACCCATGCAGTTGTTACT-3' & 5'CAATAGTGCCAGCATCTTTGGTT-3' \\
\hline BGin8567 & $\begin{array}{l}\text { Insulin-like growth factor binding } \\
\text { protein } 3 \text { (IGFBP3) }\end{array}$ & 5'-GTCTAAGCGGGAGACAGAATACG-3' & 5:-ACATTGAGGA ACTTCAGATGATTCAG-3' \\
\hline BG9663729. & $\begin{array}{l}\text { Glyceraldehyde-3-phosphate } \\
\text { dehydrogenase (GAPDH) }\end{array}$ & 5'-CCACCCACCCCAGCAA-3' & 5.GAAATTGTGAGGGAGATGCTCAGT-3' \\
\hline 11256565 & $\begin{array}{l}\text { Hydroxyprostaglandin dehydrogenase } \\
15 \text { (NAD) (HPGD) }\end{array}$ & 5'-GACCACGCCTTTGGGAAGTA-3' & 5'TCGCTTAGGGTCGTCAAGCT-3' \\
\hline \multirow[t]{2}{*}{$\mathrm{N1} 226017$} & $\begin{array}{l}\text { Sulforransferase family } 1 \mathrm{~A} \text {, phenol- } \\
\text { preferring, member } 1 \text { (SULT1A]) }\end{array}$ & 5:CCCTCAGAGTCTGCTGGATCA-3 & 5. GGAGACAACCACATCCTTTGC-3. \\
\hline & $18 \mathrm{~s}$ & 5'-AGTCCCTGCCCTTTGTACACA-3* & 5.-GATCCGAGGGCCTCACTAAAC-3 \\
\hline
\end{tabular}

'ACH: GenBank accession numbers of the CDNA fragments preseat on the microarray.

NCB1 National (center for Bbotechnology Informanon); hrtp://www. pebr.nim.nih.gow/, 
Because weight gain was similar between the groups, these results indicate that there was no difference in food intake. Furthermore, these results show that the diets did not affect normal growth.

Table 3. Mean body weights (g) per week per group.'

\begin{tabular}{|lccc|}
\hline & Week 02 & Week 13 & Week 2 \\
\hline Control group I & $18.6 \pm 0.6$ & $19.6 \pm 0.7$ & $20.3 \pm 0.7^{4}$ \\
$10 \%$ group & $18.6 \pm 1.3$ & $19.7 \pm 0.9$ & $20.2 \pm 0.8^{4}$ \\
$20 \%$ group & $19.1 \pm 0.6$ & $20.0 \pm 0.4$ & $20.8 \pm 0.3^{4}$ \\
$40 \%$ group & $18.6 \pm 0.4$ & $19.9 \pm 0.7$ & $20.6 \pm 1.0^{4}$ \\
Control group II & $17.6 \pm 0.9$ & $18.7 \pm 1.0$ & $19.4 \pm 1.4^{4}$ \\
Cauliflower group (T1) & $18.3 \pm 0.6$ & $19.5 \pm 0.6$ & $20.7 \pm 0.5^{4}$ \\
Carrots group (T2) & $17.9 \pm 0.3$ & $19.7 \pm 0.7$ & $20.2 \pm 0.8^{4}$ \\
Peas group (T3) & $18.2 \pm 0.6$ & $19.3 \pm 1.1$ & $20.3 \pm 1.1^{4}$ \\
Onions (T4) & $18.0 \pm 0.8$ & $18.9 \pm 0.7$ & $19.6 \pm 0.7^{4}$ \\
\hline
\end{tabular}

'Body weights are cxpressed as mean $\pm \mathrm{SE}$.

'Acclimatization week, animais received control diet.

Intervention period.

'Statistics: analysis of variance followed by Student's t-test. P<0.05; body weight significantly higher compared to week 0 .

By means of microarray technology, the expression levels of 602 genes were measured simultaneously. Data analysis revealed which genes were statistically significantly differentially expressed between the diet groups. In Table 4 , a list of these genes is presented together with the gene expression differences between the particular diet groups.

In the vegetable mixture diet groups, a statistically significant difference in gene expression was found for 25 diet group comparisons, representing 18 genes. Remarkably, 23 of the 25 gene expression differences are between the highest vegetable mixture group $(40 \%)$ and one of the other diet groups. According to literature review, only seven of the 18 differentially expressed genes are likely to play a role in lung cancer development. They are involved in various processes, including cell growth [insulin-like growth factor binding

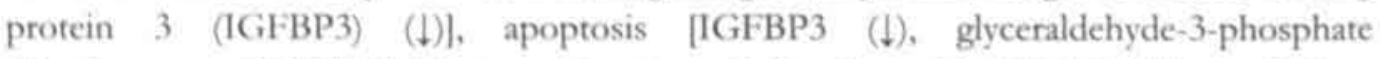
dehydrogenase (GAPDH) $(\uparrow)$, transglutaminase 2, C polypeptide (TGM2) $(\downarrow))$, metabolism |topoisomerase (DNA) II alpha $170 \mathrm{kDa}$ (TOP2A) ( $\uparrow$ ), glutamate-ammonia ligase (glutamine synthase) (GLUL) ( $\downarrow$ )], and immune response [Fc receptor, IgE, high affinity I, gamma polypeptide (FCER1G) $(\uparrow)$, cathepsin S (CTSS) ( $\downarrow$ )].

In the individual vegetable groups, a statistically significant difference in gene expression was found for 22 diet group comparisons, representing 11 genes. Three of these 11 genes were also statistically significantly different in the vegetable mixture groups, i.e. GLUL ( $\uparrow$ ), hemoglobin alpha, adult chain 1 (HBA-A1) $(\downarrow)$ and CTSS $(\downarrow)$. 


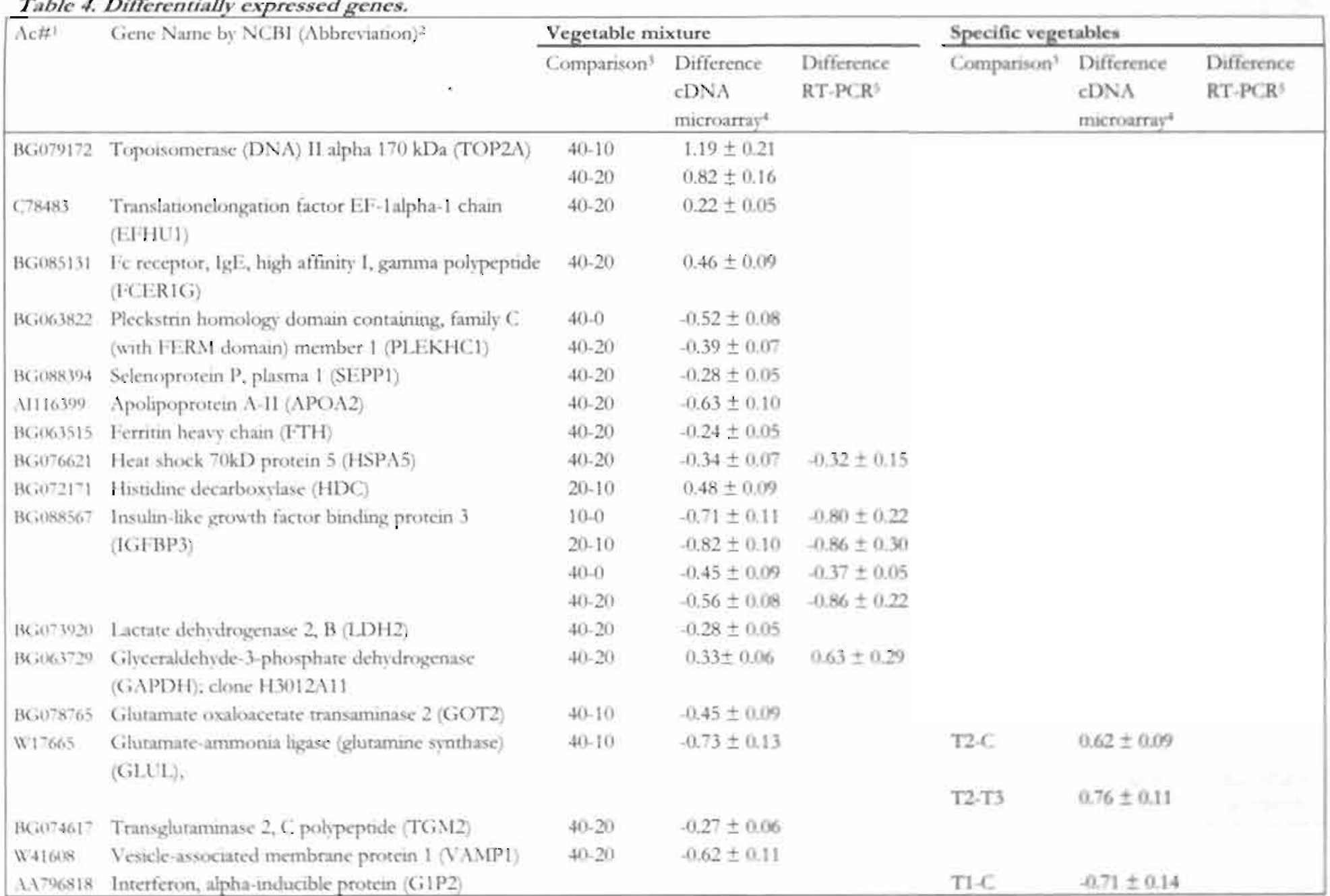


Table 4. (Continued)

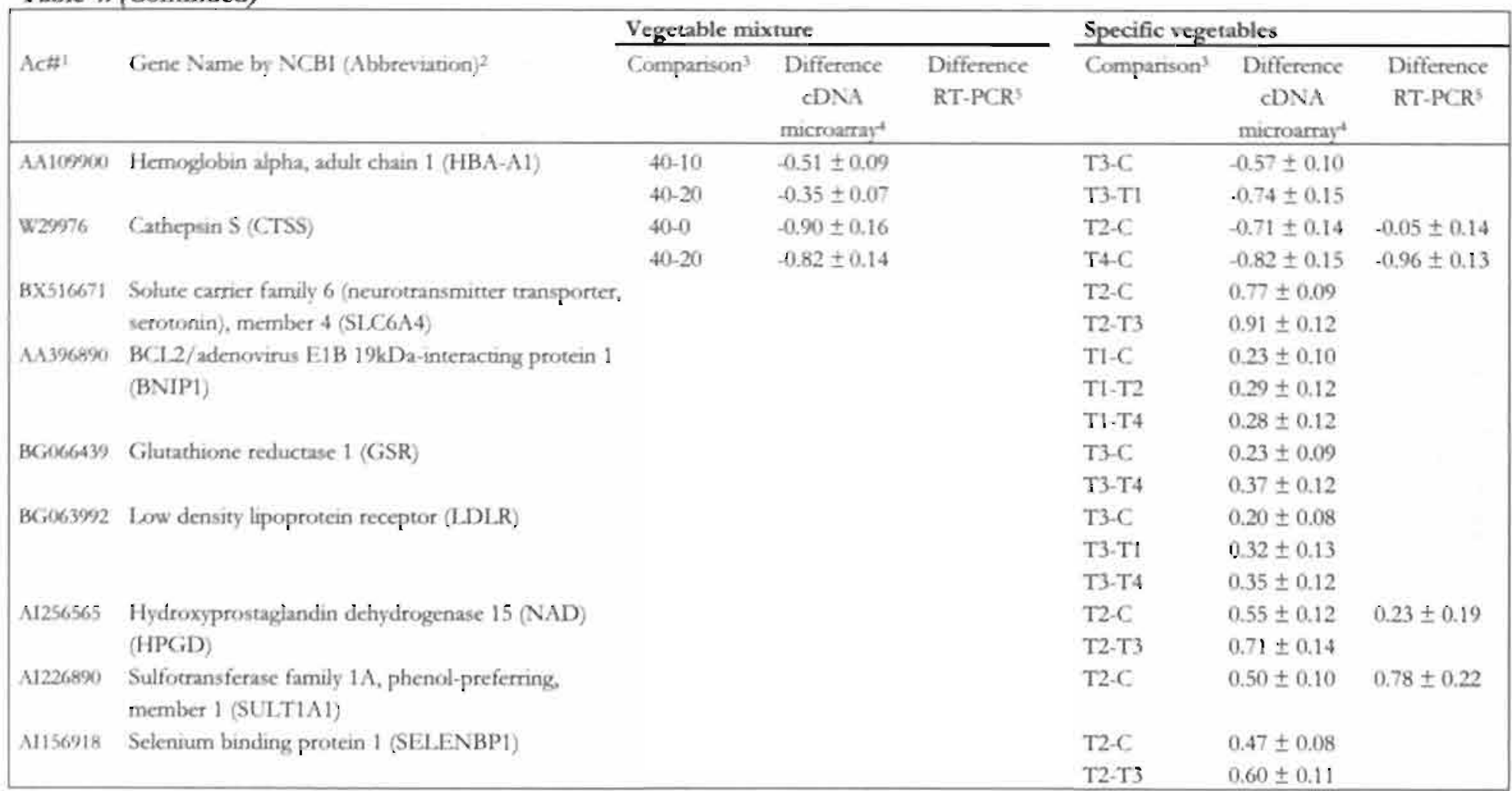

'ACA: GeaBank accesuon numbers of the CDNA fragments present on the microarrays.

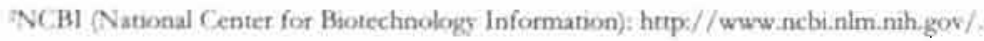

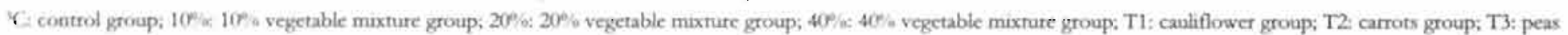
group; T4: onjons group.

'Esimated expression difference in mean Fog transformed intensity $\pm \mathrm{SE}$.

Normalized expresenon difference in mean $2 / o g$-transformed intensity \pm SE. Ten gene differences were validared. Empty cells tndicate genes which were not taken into account: 
Most (10) of the 22 statistically significant gene expression differences occurred in the carrots group, representing six genes [GLUL ( $\uparrow$ ), CTSS $(\downarrow)$, solute carrier family 6 (neurotransmitter transporter, serotonin), member 4 (SLC6A4) $(\uparrow)$, hydroxyprostaglandin dehydrogenase 15 (NAD) (HPGD) ( $\uparrow$ ), sulfotransferase family $1 \mathrm{~A}$, phenol-preferring, member 1 (SULT1A1) ( $\uparrow$ ) and selenium binding protein 1 (SELENBP1) ( $\uparrow$ )]. CTSS was the only gene, which was modulated by more than one individual vegetable. In addition to carrots, onions had an effect on the expression of this gene, which was also the single gene effect occurring in this group. Second to the carrots, peas caused seven gene expression differences, accounting for three genes, including HBA-A1 ( $\downarrow)$, glutathione reductase 1 $($ GSR) $(\uparrow)$ and low density lipoprotein receptor (I.DLR) $(\uparrow)$. Finally, cauliflower modulated the expression of $\mathrm{BCl} 2$ /adenovirus E1B 19kDa-interacting protein 1 (BNIP1) ( $\uparrow$ ) and interferon, alpha-inducible protein (G1P2) ( $\downarrow$ ), representing four gene expression differences. According to literature review, eight of these 11 genes might play a role in lung cancer development. In Figure 1, the estimated differences of the genes which are likely to play a role in prevention of lung cancer risk, according to literature review, per vegetable group as compared to the control group (set to 0 ) are illustrated.

Ten gene expression differences, representing five genes responsive to vegetables, were validated by real-ume KI-PCK. The results are shown in Table 4. These data prove the results obtained by microarray analyses and verify the reliability of this cDNA microarray approach to identify genes that are modulated by vegetables.
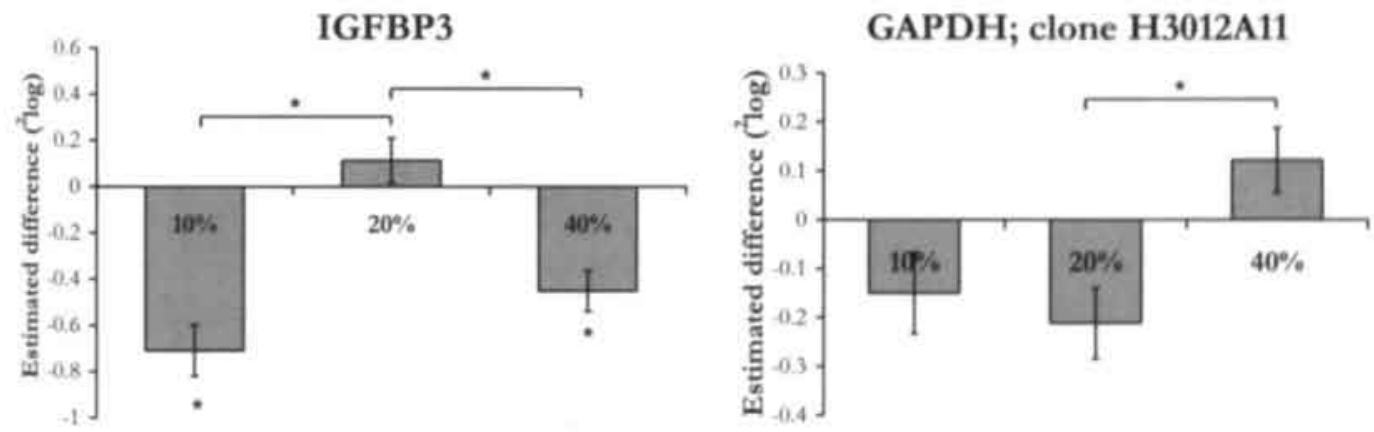

Figure 1. Estimated difference in mean 7 og-transformed intensity \pm SE per diet group ( $x$-axis) for all identified differentially expressed genes from Table 4 (10\%: $10 \%$ vegetable mixture group; $20 \%$ o: $20 \%$ vegetable mixture group; $40 \%: 40 \%$ vegetable mixture group; T1: cauliflower group; T2: carrots group; T3: peas group: T4: onions group). The control diet group intensary (set to 0 ) serves as the reference, IGFBP3: insulin-like growth factor bending protein 3; GAPDH: plyceraldehyde-3phosphate d chydrogenase, TGM2 transglutaminase 2, C polypeptide, TOP2A: topotsomerase (DNA) II alpha $170 \mathrm{kDa}$ GLUI: glutamate-ammonia bigse (glutamine synthase); FCFRIG: Fe receptor, IgE, hiyh affinity 1, gamma polypeptide; CTSS: cathepsin S; HPGD: hydroxyprostaglandin dehydrogenase 15 (NAD); SULT1A1: sulfotransferase family 1A, phenolpreferring, member 1; SEL.ENBP1: selenium binding protein 1; GSR: glutathone reductase 1; LDI.R: low density lipoprotein receptor; BNIP1: BC12/adenovirus E1B 19kDa-interacting protem 1; G1P2: interferon, alpha-inducible protein. Astenks without connection lines indicate statistically significant difference between vegetable group and control group. See Table 4 for other statistical details. 

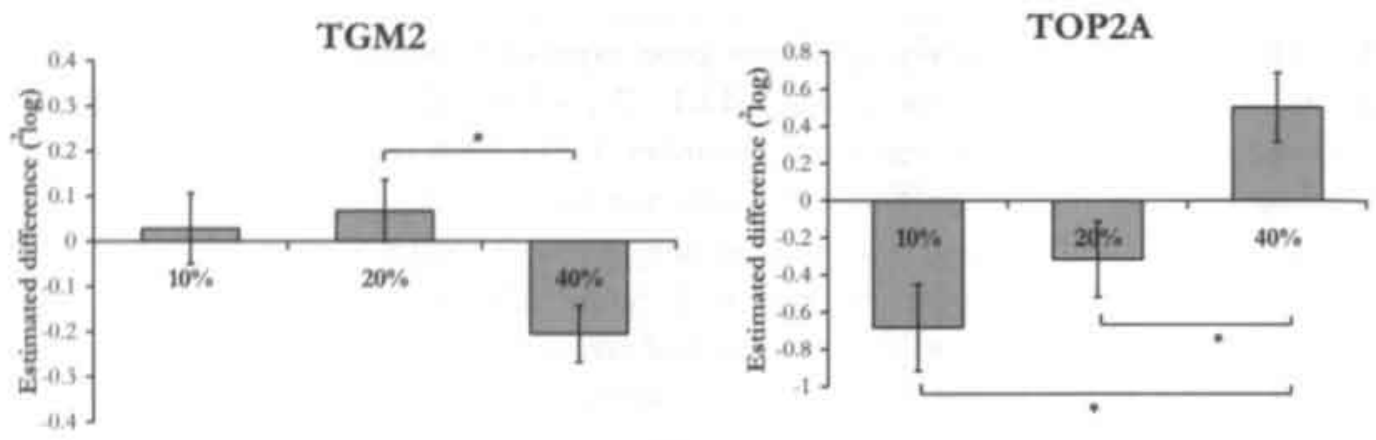

FCER1G
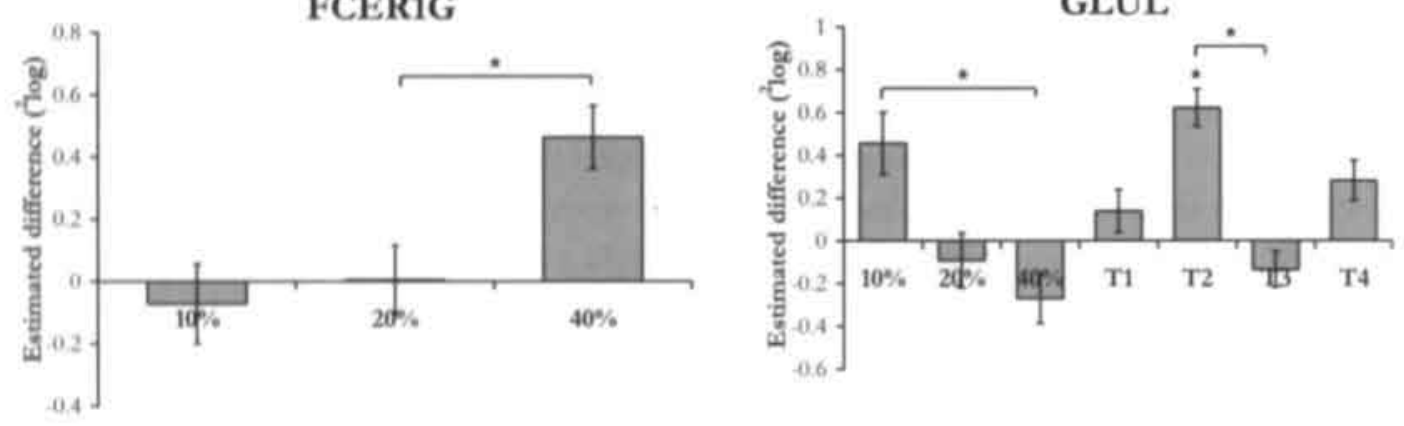

CTSS
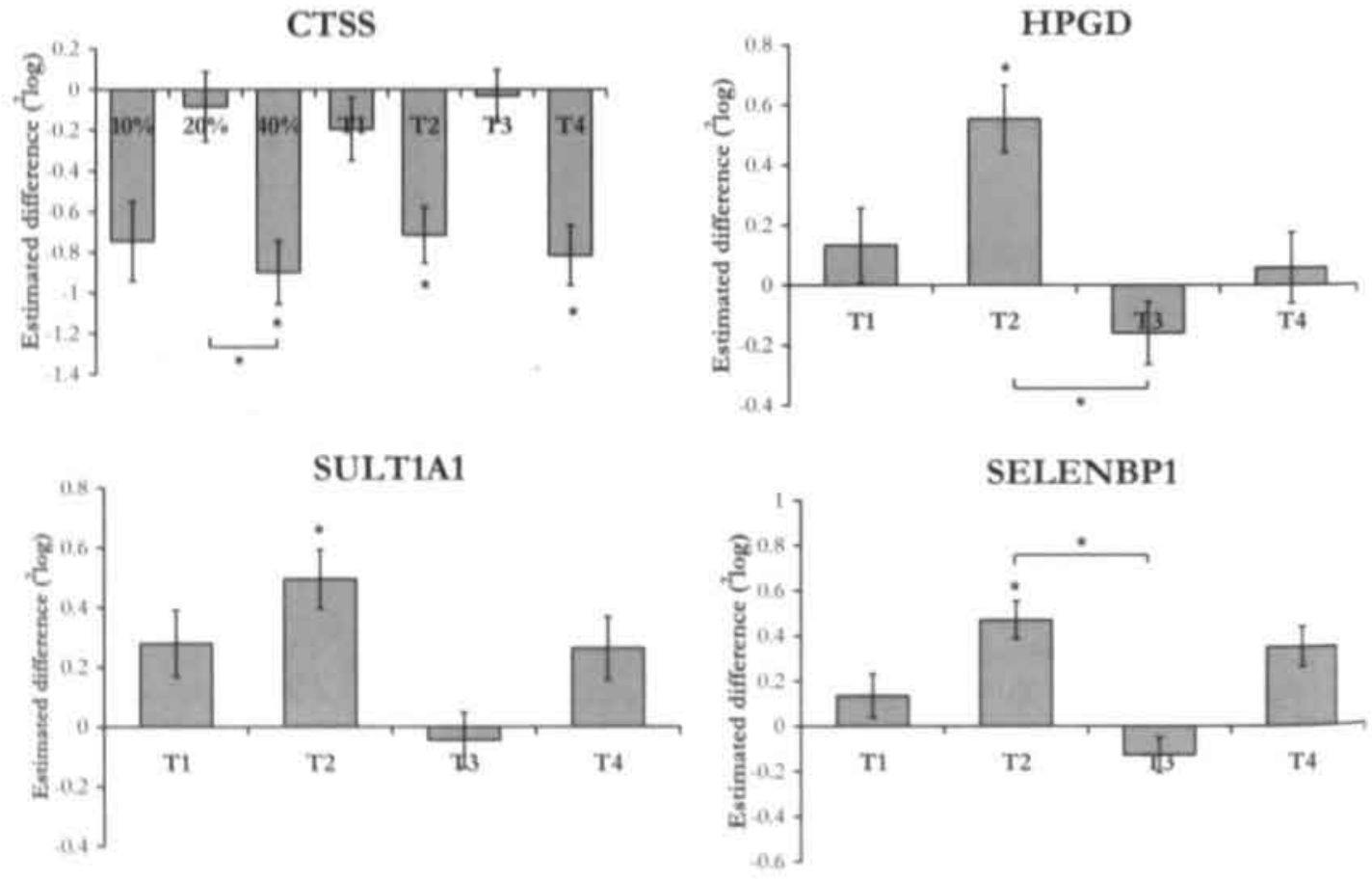

Figure 1. (Continued) 

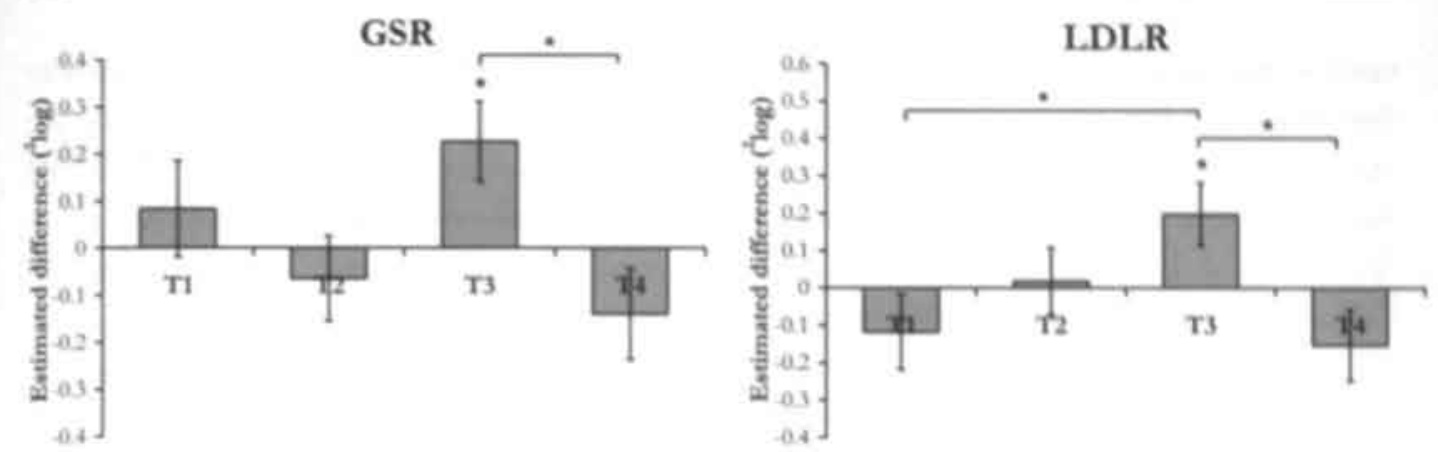

BNIP1

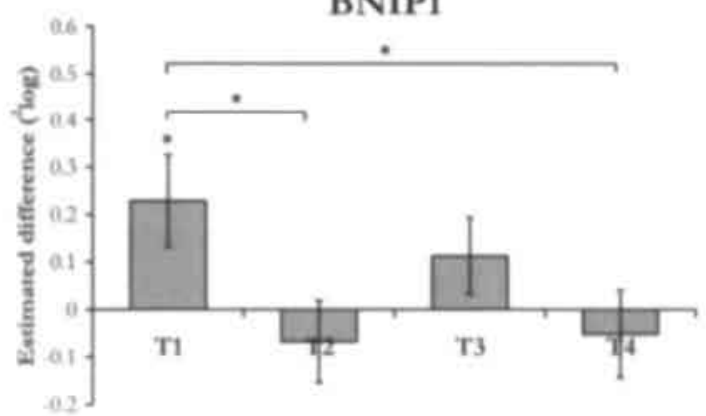

Figure 1. (Continued)

\section{Discussion}

In this study, the effect of different doses of a vegetable mixture and of the individual vegetables present in the mixture on gene expression changes in the lung of female C57BL6 mice was investigated by means of microarray technology. First, the effects of the vegetable mixture will be discussed; next, the contribution of the four individual vegetables on gene expression changes in lung cancer risk preventive pathways.

At least 13 genes, which were modulated by one or more of the vegetable groups, could play a role in different mechanisms involved in preventing lung carcinogenesis. Table 5 summarizes the effects of the vegetable groups on the expression of these genes in relation to possible effects on prevention of lung cancer risk.

In the vegetable mixture groups, seven genes were modulated which could be involved in preventing lung cancer risk. One of these genes is concerned with cell growth, i.e. IGFBP3. The protein encoded by this gene belongs to one of the six members of the IGFBP family and regulates the activity of insulin-like growth factor 1 (IGF-1), thereby inhibiting the mitogenic and antiapoptotic action of IGF-1. IGFBP3 also inhibits cell growth and induces apoptosis independent of IGF-1 by inhibiting the p13K/Akt $/ \mathrm{PKB}$ and MAPK signaling pathways. ${ }^{21}$ In mice, 1GFBP-3 inhibited the growth of lung tumors 
and in humans, a higher IGFBP-3 level in serum was associated with lower lung cancer risk. In in vitro and in vivo, the expression of IGFBP3 was induced by dietary factors like lycopene, retinoic acid, flavonoids and green tea. ${ }^{2}$ However, in the present study, IGFBP3 was downregulated in the $10 \%$ and $40 \%$ vegetable mixture groups. Possible other constituents than lycopene, retinoic acid and flavonoids are present in the vegetables which have a downregulating effect on the expression of this gene.

Next to IGFBP3, two other apoptosis genes were modulated in this study, i.e. GAPDH and TGM2. Besides playing an important role in glycolysis in the cytosol, GAPDH has diverse cellular functions, one of the most intriguing is likely to be the induction of apoptosis $^{23}$, although at present the mechanism of action is unknown. ${ }^{24}$ TGM2 covalently cross-links cellular proteins and polyamines. Increased TGM2 expression is associated with apoptosis, probably by induction of massive $\mathrm{Ca}^{2+}$-mediated intracellular cross-linking ${ }^{25}$ Furthermore, it has been found that TGM2 was upregulated in bronchial epithelial cells by retinoic acid, which has a role in cancer treatment and prevention by inducing growth suppression. ${ }^{26}$ GAPDH was upregulated and TGM2 downregulated in the highest vegetable group in the current study. The results of the modulation by the vegetable mixture on IGFBP3, GAPDH and TGM2 are expected to be induction of cell growth and reduction of apoptosis, which are not in line with the proposed hypothesis of cancer risk prevention by vegetables.

Two genes, which were modulated by the vegetable mixture, are involved in metabolism, i.e. TOP2A and GLUL. TOP2A codes for an essential nuclear enzyme that carries out topological changes in DNA and plays important role in DNA replication, transcription and chromosome condensation.27 Overexpression of TOP $2 \mathrm{~A}$ might contribute to accelerated cell proliferation, abortive G2 cell cycle checkpoints, tumor dedifferentiation and sensitivity to anticancer drugs. Although downregulation of TOP2A was reported by genistein 28 , an isoflavone present in soybeans, in the present study, TOP2A was up-regulated in the highest vegetable dose group. The protein encoded by GLUL is responsible for catalyzing the formation of glutamine from glutamate using ammonium ions and ATP to maintain glutamine homeostasis. It plays an important role in controlling body $\mathrm{pH}$ and in removing ammonia from the body. It was shown that GLUL expression is increased in the lungs in response to trauma or infection. There is a paradox that GLUL expression is decreased in tumor cells and that large amounts of glutamine are required for tumor cell growth. It is hypothesized that reactivation of GLUL expression would have growth suppressive effects on tumor cells, however, this has not yet been resolved. ${ }^{29}$ In the present study, GLUL expression was downregulated in the $40 \%$ vegetable mixture group, but upregulated in the carrots vegetable group. The modulation by the vegetable mixture on TOP2A and GLUL gene expression might result in increased cell proliferation and, again, these results are not in favor of lung cancer risk prevention. 
Table 5. Presentation of differentially expressed genes likely to be involved in lung cancer preventive pathways, the direction of the effect in the vegetable groups, and the theoretical effect of this on cancer risk.

\begin{tabular}{|c|c|c|c|c|c|c|c|c|c|}
\hline \multirow[t]{2}{*}{ Abberviation' } & \multicolumn{4}{|c|}{ Vegetable mixture groups } & \multicolumn{5}{|c|}{ Individual vegetable groups } \\
\hline & $10 \%$ & $20 \%$ & 4006 & $\begin{array}{l}\text { Efficet on } \\
\text { cancer risk }\end{array}$ & Cauliflower & Carrots & Peas & Onions & $\begin{array}{l}\text { Effect on } \\
\text { cancer nak }\end{array}$ \\
\hline IGFAP3 & - & & - & + & & & & & \\
\hline GAPDH & & & + & - & & & & & \\
\hline TGM2 & & & - & + & & & & & \\
\hline TOP2A & & & + & + & & & & & \\
\hline FCERIG & & & + & - & & & & & \\
\hline GuL. & & & - & + & & + & & & - \\
\hline CTSS & & & - & + & & - & & - & + \\
\hline HPGD & & & & & & + & & & + \\
\hline SULTIAI & & & & & & + & & & - \\
\hline SEIENBP1 & & & & & & + & & & - \\
\hline GSR & & & & & & & + & & - \\
\hline IDIR & & & & & & & + & & + \\
\hline BNIP1 & & & & & + & & & & - \\
\hline
\end{tabular}

NCBI (National Center for Biotechnology Information): hrtp:/ /www.ncturnim.nith gow /

Two genes involved in immune response were modulated in the $40 \%$ vegetable mixture group, i.e. FCER1G and CTSS. FCER1G is one of the Fc receptors, which plays a role in effective immunity against malignant cells; probably by enhancement of Fc receptor mediated antibody-dependent cellular cytotoxicity resulting in tumor cytotoxicity. ${ }^{30}$ CTSS has been characterized as a key enzyme in major histocompatibility complex class II mediated antigen presentation. Increased expression of CTSS in lung tumors was associated with better survival probability for lung cancer patients. The high expression could initiate modeling of antigen presentation and consequently the response to tumor antigens. ${ }^{31}$ However, increased CTSS has also been associated with autoimmune diseases. Inhibition of CTSS alters autoantigen presentation and inhibits development of organspecific autoimmunity. ${ }^{32}$ FCER1G was upregulated, whereas CTSS was downregulated in the $40 \%$ vegetable mixtures group. CTSS was also downregulated in the individual carrots and onions vegetable group, which could be responsible for the observed effect in the $40 \%$ vegetable mixture group. The net effect of the modulation of FCER1G and CTSS by the vegetable mixture on immune response is not clear, because the effects on the immune system are opposite for these genes.

Summarizing, the effects on gene expression changes by the vegetable mixture, five out of the seven modulated genes involved in lung cancer preventive mechanisms are expected to result in increased lung cancer risk.

With respect to the individual vegetables, most of the statistically significant gene expression differences occurred in the carrots vegetable group. In addition to the already 
discussed genes GLUL $(\uparrow)$ and CTSS $(\downarrow)$, also SLC6A4 $(\uparrow)$, HPGD $(\uparrow)$, SULT1A1 $(\uparrow)$ and SELENBP1 ( $\uparrow$ gene expressions were modulated. No role for SLC6A4 in lung cancer preventive mechanisms has been described. HPGD encodes for an enzyme, which metabolizes a number of prostanoids and non-prostanoid compounds. The products of the non-prostanoids compounds are generally highly reactive $\alpha, \beta$-unsaturated aldehydes and ketones, which may cause carcinogenesis. HPGD is a target for many non-steriodal anti-inflammatory drugs, including cyclooxygenase-2 inhibitors. Also phytophenolic compounds such as curcumin, flavonoids and epigallocatechin gallate were found to inhibit colonic HPGD enzyme activity. ${ }^{33}$ Modulation of lung HPGD by dietary compounds has not been reported yet. The protein product of SULT1A1 belongs to a superfamily of enzymes, which catalyze the sulfate conjugation of many hormones, neurotransmitters, drugs and xenobiotic compounds. They play an important role in chemical defense mechanisms against various xenobiotics but also bioactivates many dietary procarcinogens, including heterocyclic aromatic amines implicated in the pathogenesis of colorectal cancer. ${ }^{34}$ In lung cancer, however, it was shown that a higher activity of this enzyme in the lung was associated with a decreased lung cancer risk. ${ }^{35}$ SELENBP1 encodes for a protein which belongs to the selenium containing proteins. Little information is available about the function of this protein in lung cancer or other cancers. Chen et al (2004) have found a that reduced selenium-binding protein 1 expression is associated with poor outcome in lung adenocarcinomas, possibly by increasing cell proliferation and decreasing differentiation. ${ }^{36}$ As indicated by literature review, no information is available about gene expression modulation in the lung by consumption of carrots. However, few studies have investigated the effect of $\beta$-carotene and/or vitamin $A$ on the expression of genes in the lungs of rats. It was found that pharmacological (i.e. high dose) $\beta$-carotene supplementation in rats resulted in increased expression of activator protein 1, proliferating cell nuclear antigen and cyclin D1, and reduced expression of retinoic acid receptor $\beta$ in rat lung tissue. Modulations of these genes were associated with increased keratinized squamous metaplasia. No modulations were found after physiological (i.e. low dose) $\beta$-carotene supplementation. ${ }^{37}$ Borràs et al (2003) have reported reduced expression of p 53 and p 21 and increased expression of c-jun and cyclin DI in lung tissue of rats supplemented with vitamin A. ${ }^{38}$ In the present study, the dose of $\beta$-carotene is expected to be comparable with physiological doses, because it is present in the biological source and not supplemented in high amounts. This could explain the relatively beneficial effects of carrots on gene expression changes in the mouse lung.

Summarizing the effect of carrots on gene expression changes, five out of six modulated genes could be involved in lung cancer protective mechanisms; three (GLUL. SULT1A1 and SELENBP1) out of these five are affected in such a way that lung cancer protective mechanisms could be expected. 
In addition to modulation of CTSS by carrots, onions were able to downregulate the expression of this gene. No other genes were affected by the onions. No information on lung cancer risk prevention for onions or other allium vegetables is present from epidemiological studies. In nitro and in nive, a protective effect of allium compounds on lung cancer risk was reported. ${ }^{\text {y. }}$, with regard to modulation of gene expression, the allium compound flavone induced $\mathrm{p} 21$ gene expression in human lung adenocarcinoma cells. ${ }^{10}$ The present study is the first in which onions modulated gene expression in uive.

Three genes were modulated in the peas group, i.e. HBA-A1 ( $\downarrow)$, GSR $(\uparrow)$ and LDLR $(\uparrow)$. No lung cancer preventive pathways in which HBA-A1 could play a role are currently known. GSR codes for an enzyme which reduces oxidized glutathione. Glutathione is involved in the antioxidative defense system against endogenous and exogenous pro-oxidants. Radicals and reactive oxygen species are inactivated by e.g. catalase, superoxide dismutase and glutatione peroxides, which oxidize glutathione in the same step. Dietary broccoli has been shown to increase GSR expression in the liver, kidney and colon. ${ }^{41}$ This is the first study in which vegetables were demonstrated to induce GSR expression in the lungs and thereby to provide the lungs with more reduced oxidized glutathione which could result in an improved antioxidative defense in the lungs. The protein product of LDLR is a cell surface receptor that plays an important role in cholesterol homeostasis. Mammalian cells can obtain cholesterol by de novo synthesis (minor pathway) or by the uptake of lipoprotein derived cholesterol via the LDI. receptor (major pathway). It has been shown that tumor cell lines have higher LDL receptor activity than the corresponding normal cells. Furthermore, LDL uptake has been shown to be higher in lung tumor tissue than in the corresponding tissue. The reasons for this are not clear, but it is hypothesized that cholesterol is needed for cell growth. ${ }^{42}$

Taken all this together, two out of three genes modulated by the peas could be involved in lung cancer preventive pathways; only the effect on GSR could lead to lung cancer risk prevention by inducing antioxidative defense.

Finally, cauliflower was able to modulate the expression of the genes BNIP1 ( $\uparrow$ ) and GIP2 ( $\downarrow$ ). Only BNIP1 is likely to be involved in lung cancer preventive pathways. No modulation of BNIP1 mRNA or enzyme activity by vegetables or nutrition has been described yet. The protein product of the BNIP1 gene interacts with members of the $\mathrm{BCl} .2$ family, which consists of pro-apoptotic (such as BAX) and anti-apoptotic (such as $\mathrm{BCL}$ 2) proteins. It is suggested that BNIP1 has pro-apoptotic properties, by interacting with BCL.2 and BCL.2L.1.43, ${ }^{4}$ It was shown that BNIP1 mRNA was downregulated in mouse lung tumor tissue, possibly leading to reduced apoptosis of tumor cells. ${ }^{45}$ However, the precise function of BNIP1 is unclear. ${ }^{43}, 44$ Upregulation of BNIP1 by cauliflower could lead to increased apoptosis, which is generally regarded as a protective mechanism against cancer by removing genetically damaged cells before they can undergo clonal expansion. 
Comparing the results from the vegetable mixtures groups with the individual vegetables on gene expression changes, it is clear that in general, other genes are modulated by the vegetables mixture as compared to the individual vegetables. Only three genes were modulated by both the vegetable mixture and one or more of the individual vegetable groups. The gene expression differences occurring in the vegetable mixture groups can not be explained (except for CTSS and HBA-A1) by the effects in the individual vegetables. It could be that the amount of bioactive compounds in individual vegetables is too small to modulate the expression of genes. Possible, the combined effect of micronutrients from different vegetables could be synergistic or additive and therefore large enough to exert a gene expression effect.

In the present study, the complete mouse lung was used for gene expression analysis. The lungs contain a heterogeneous population of cells, such as macrophages, various epithelial cells and smooth muscle cells. It can not be excluded that the effects on gene expression in specific lung cancer target cells by vegetables are diluted by the effects on gene expression in other cells. Isolation, however, of specific target cells has limitations as well. Protocols for the isolation of homogeneous populations of specific cells require a lot of time, thereby risking degradation of mRNA, and furthermore, these protocols comprise enzymatic digestion or agitation and centrifugation, which could also influence gene expression patterns. The relatively new technology of laser capture microdissection can overcome these drawbacks, however, this technology is still in its infancy. ${ }^{46}$

In this study, 13 genes were modulated which could be involved in lung cancer risk preventive pathways. From Table 5, it becomes clear that for the vegetable mixture groups only 2 out of 7 , and for the individual vegetables 5 out of 8 are modulated in such a way that lung cancer preventive pathways could be stimulated; the other gene modulations are expected to enhance lung cancer risk. These results do not provide a strong indication of favorable vegetable-induced gene expression modulations which could result in reduced lung cancer risk. But, it is very difficult to estimate the contribution of each gene expression modulation in carcinogenesis. The net effect of the gene expression modulations by the vegetables could still be in favor of lung cancer risk prevention. It seems that individual vegetables have a higher potential of modulating genes in favor of lower lung cancer risk, in stead of a combined effect. Furthermore, carrots modulated most gene expressions, and most of these effects were also in support of lung cancer risk prevention. These results are in line with the prospective epidemiological studies in which was shown that particularly dark green and yellow-orange vegetables, rich in $\beta$-carotene and vitamin $\mathrm{E}$, are the specific types of vegetables, which have the most available and consistent evidence in reducing lung cancer risk. The current study provides more insight into the genetic mechanisms by which vegetables, in particular carrots, can prevent lung cancer risk. 


\section{References}

1. World Health Organization \& Intemational Agency for Research on Cancer. World Cancer Report. Lyon: IARCPress, 2003.

2. Omenn GS. Chemoprevention of lung cancer: the nse and demise of beta-carotene. Annu Rev Public Health 1998:19:73-99.

3. World Cancer Research Fund. Food, Nutrition and the Prevention of Cancer a global perspective. Chapter 4.5. Lung Washington: BANTA Book group, 1997.130-147.

4. Peto R, Doll R, Buckley JD, Spom MB. Can dietary beta-carotene materially reduce human cancer rates? Nature 1981:290:201-8.

5. Steinmetz KA, Potter JD. Vegetables, fruit, and cancer. II. Mechanisms. Cancer Causes Control $1991: 2: 427-42$

6. Thompson HJ, Heimendinger J, Hacgele A, Sedlacek SM, Gillerte C, O'Neill C, Wolfe P, Conry C. Effect of increased vegetable and frut consumption on markers of oxidative cellular damage. Carcinogenesis 1999;20:2261-6.

7. Zino S, Skeaff M, Williams S, Mann J. Randomised controlled trial of effect of fruit and vegetable consumption on plasma concentranions of lipids and antioxidants. Bmi 1997;314:1787.91.

8. Nishino H. Cancer prevention by natural carotenoids. J Cell Biochem Suppl 1997:27:86-91.

9. Dutta $\mathrm{A}$, Durta SK. Vitamin $\mathrm{E}$ and its role in the prevention of atherosclerosis and carcinogenesis: a review. J Am Coll Nutr 2003;22:258-68.

10. Omenn GS, Goodman G, Thornquist M, Grizzle J, Rosenstock L, Barnhart S, Balmes J, Chemiack MG, Cullen MR, Glass A, et al. The beta-carotene and retinol efficacy trial (CARET) for chemoprevention of lung cancer in high risk populations: smokers and asbestos-exposed workers. Cancer Res 1994:54:2038s-2043s.

11. The effect of vitamin $\mathrm{E}$ and beta carotene on the incidence of lung cancer and other cancers in male smokers. The Alpha-Tocopherol, Beta Carotene Cancer Prevention Study Group. N Engl J Med 1994;330:1029-35.

12. Kelley MJ, MeCrory DC. Prevention of lung cancer: summary of published evidence. Chest 2003;123:50S-59S.

13. Ziegler RG, Mayne ST, Swanson CA. Nutrition and lung cancer. Cancer Causes Control 1996;7:157. 77.

14. Barrett JC, Kawasaki ES. Microarrays: the use of oligonucleotides and eDNA for the analysis of gene expression. Drug Discov Today 2003;8:134 41 .

15. Hasseman J, Chen E, Yang I, Quackenbush J. Aminoallyl labeling of RNA for microarrays. hrtp://www.tigr.org/tdb/micoarray/protocolsTIGR.shtml (accessed June 21, 2001), 2001.

16. Churchill GA. Fundamentals of experimental design for cDNA microarrays. Nat Genet 2002;32 Suppl 2:490-5.

17. Kerr MK. Destgn considerations for efficient and effective microarray studies. Biometrics 2003;59:822-828.

18. Kerr MK, Martin M, Churchill GA. Analysis of variance for gene expression microarray data. Journal of Computational Biology 2000;7:819-837.

19. Wolfinger RD, Gibson G, Wolfinger ED, Bennett 1, Hamadeh H, Bushel P, Afshari C, Paules RS. Assessing gene significance from eDNA microartay expression data via mixed models. J Comput Biol 2001;8:625-37. 
20. Neter J, Kutner MH, Nachtsheim CJ, Wasserman W. Applied Linear Statistical Models (Fourth Edition). Mass, 1996.

21. Lee HY, Chun KH, Liu B, Wichle SA, Cristiano RJ, Hong WK, Cohen P, Kurie JM. Insulin-like growth factor binding protein-3 inhibits the growth of non-small cell lung cancer. Cancer Res 2002;62:3530-7.

22. Ali O, Cohen P, Lee KW. Epidemiology and biology of insulin-like growth factor binding protein-3 (GFBP-3) as an anti-cancer molecule. Horm Metab Res 2003;35:726-33.

23. Ishitani R, Tajima H, Takata H, Tsuchiya K, Kuwae T, Yamada M, Takahashi H, Tarton NA, Katsube N. Proapoptotic protein glyceraldehyde-3-phosphate dehydrogenase: a possible site of action of antiapoptotic drugs. Prog Neuropsychopharmacol Biol Psychiatry 2003;27:291-301.

24. Berry MD, Boulton AA. Glyceraldehyde-3-phosphate dehydrogenase and apoptosis. J Neurosei Res 2000;60:150-4.

25. Griffin M, Casadio R, Bergamini CM. Transglutaminases: nature's biological glues. Biochem J 2002;368:377-96.

26. Ma Y, Koza-Taylor PH, DiMattia DA, Hames L, Fu H, Dragnev KH, Turi T, Beebe JS, Freemantie SJ, Dmitrovsky E. Microarray analysis uncovers retinoid targets in human bronchial epithelial cells. Oncogene 2003;22:4924-32.

27. Watt PM, Hickson ID. Structure and function of type II DNA topoisomerases. Biochem J 1994;303 (Pt 3):681-95.

28. Kurzer MS, Xu X. Dietary phytoestrogens. Annu Rev Nutr 1997;17:353-81.

29. Schraml P, Shipman R, Colombi M, Ludwig CU. Identification of genes differentially expressed in normal lung and non-small cell lung carcinoma tissue, Cancer Res 1994;54:5236-40.

30. Clynes R, Takechi Y, Moroi Y, Houghton A, Ravetch JV. Fe receptors are required in passive and active immunity to melanoma. Proc Natl Acad Sci U S A 1998;95:652-6.

31. Kos J, Sekimik A, Kopitar G, Cimerman N, Kayser K, Stremmer A, Fiehn W, Werle B. Cathepsin S in tumours, regional lymph nodes and scra of patients with lung cancer: relation to prognosis. $\mathrm{Br}$ J Cancer 2001;85:1193-200.

32. Sacgusa K, Ishimaru N, Yanagi K, Arakaki R, Ogawa K, Saito I, Katunuma N, Hayashi Y. Cathepsin $S$ inhibitor prevents autoantigen presentation and autoimmunity. J Clin Invest 2002;110:361-9.

33. Cho $\mathrm{H}$, Tai $\mathrm{HH}$. Inhibition of $\mathrm{NAD}+$ dependent 15-hydroxyprostaglandin dehydrogenase (15. PGDH) by cyclooxygenase inhibitors and chemopreventive agents. Prostaglandins Leukot Essent Fatty Acids 2002;67:461-5.

34. Bamber DF, Fryer AA, Strange RC, Elder JB, Deakin M, Rajagopal R, Fawole A, Gilissen RA, Campbell FC, Coughtrie MW. Phenol sulphotransferase SULT1A1*1 genotype is associated with reduced risk of colorectal cancer. Pharmacogenetics 2001;11:679-85.

35. Liang G, Miao X, Zhou Y. Tan W, Lin D. A functional polymorphism in the SULT1A1 gene (G638A) is associated with risk of lung cancer in relation to tobacco smoking. Carcinogenesis 2004:25:773-8.

36. Chen G, Wang H, Miller CT, Thomas DG, Gharib TG, Misek DE, Giordano T], Orringer MB, Hanash SM, Beer DG. Reduced selenium-binding protein I expression is associated with poor outcome in lung adenocarcinomas. J Pathol 2004:202:321-9.

37. Liu C, Wang XD, Bronson RT, Smith DE, Krinsky NI, Russell RM. Effects of physiological versus pharmacological beta-carotene supplementation on cell proliferation and histopathological changes in the lungs of cigarette smoke-exposed ferrets. Carcinogenesis 2000;-21:2245-53. 
38. Borras E, Zaragoza R, Morante M, Garcia C, Gimeno A, Lopez-Rodas G, Barber T, Miralles VJ. Vina JR, Torres L In vivo studies of altered expression parterns of p53 and proliferative control genes in chronic vitamin A deficiency and hypervitaminosis. Eur J Biochem 2003;270:1493-501.

39. Bai F, Matsui T, Ohtani-Fupita N, Matsukawa Y, Ding Y, Sakai T. Promoter activation and following induction of the p21/WAF1 gene by flavone is involved in G1 phase arrest in A549 lung adenocarcinoma cells. FEFS Lett 1998;437:61-4.

40. Khanduja KI, Gandhi RK, Pathania V, Syal N. Prevention of N-nitrosodiethylamine induced lang tumorigenesis by ellagic acid and quercetin in mice. Food Chem Toxicol 1999;37:313-8.

41. Vang $\mathrm{O}$, Rasmussen BF, Andersen $\mathrm{O}$. Combined effects of complex mixtures of potentially anticarcinogenic compounds on antioxidant enzymes and carcinogen metabolizing enzymes in the rat. Cancer Letr 1997;114:283-6.

42. Gueddari N, Favre G, Marmouget C, Soula G, Le Gaillard F. Involvement of tyrosine kinase activity in the low-densiry lipoprotein receptor expression in human lung adenocarcinoma cell line A549. Biochimic 1996;78:874-81.

43. Zhang $\mathrm{H}$, Heim J, Meyhack B. Novel BNIP1 variants and their interaction with BCI 2 family members. FEBS lett 1999;448:23-7.

4. Yasuda M, Chinnadura G. Functional identification of the apoptosis effector BH3 domain in cellular protein BNIP1. Oncogene 2000;19:2363-7.

45. Bonner AE, Lemon W], Devereus TR, Lubet RA, You M. Molecular profiling of mouse lung rumors: association with tumor progression, lung development, and human lung adenocarcinomas. Oncogene 2004;23:1166-76.

46. Emmert-Buck MR, Bonner RF, Smith PD, Chuaqui RF, Zhuang Z, Goldstein SR, Weiss RA, Liotta LA. Laser capture microdissection. Science 1996;274:998-1001. 



\section{Chapter 7}

Summary and general discussion 


\section{Summary and General Discussion}

Food preparation and dietary habits are very relevant exogenous factors affecting cancer risk. The most consistent finding on diet as a determinant of cancer risk is the association between consumption of vegetables and fruit and reduced risk of several cancers. Convincing epidemiological evidence exists for cancer of the gastrointestinal tract (oral cavity, pharynx, esophagus, stomach, colon and rectum) and of the respiratory tract (lung). ${ }^{1}$ In terms of incidence, lung cancer is the most common cancer worldwide, closely followed by cancer of the colorectum as the third most frequent. ${ }^{2}$ Diets containing considerable amounts of a variety of vegetables $(>400 \mathrm{~g} /$ day) may reduce these types of cancer by 45 respectively 40 percent (range $30-50$ ). ${ }^{3}$ The mechanisms by which vegetables exert their beneficial effects are various and studied in experimental systems. However, whether vegetables exert their effect by affecting genetic pathways is still mostly unknown, because only few studies have investigated the effect of vegetables and vegetable constituents on gene expression changes in target organs. Nowadays, microarray technology can be used to investigate the effect of a specific diet on the expression of multiple genes or proteins in a single experiment. It can help to identify the genetic mechanisms by which vegetables affect cancer risk at the molecular level.

The hypothesis investigated in the present thesis is that an important contribution of the anticarcinogenic effects of vegetables in the colorectum and lung is through modulating the expression of genes involved in biological and genetic pathways that are relevant for chemical carcinogenesis. Therefore, the aim is to provide more insight into the molecular mechanisms by which vegetables exert their anticarcinogenic effects, by focusing on the effects at the genome level in the relevant target cells.

The model of colorectal cancer (CRC) proposed by Fearon and Vogelstein consists of successive genetic changes, in which a number of genes are involved, including APC (adenopolyposis coli), K-RAS, DCC (deleted in colorectal cancer) and p53. ${ }^{4}$ During the last decade, additional genetic events and specific molecular pathways have been identified. It became clear that the intact or mutated key molecules of the Vogelstein model interact and form a network of molecular events affecting additional genes. ${ }^{5,6}$ These genetic pathways and the involved genes are obvious molecular targets for the protection against CRC by vegetables. To identify the genes which are modulated in vivo in colorectal mucosa from humans by vegetables, the study described in Chapter 2 was carried out. In this human dietary intervention study the effect of a $50 \%$ decreased $(=75 \mathrm{~g} /$ day $)$ or doubled intake $(=300 \mathrm{~g} /$ day) of a mixture of vegetables (cauliflower, carrots, peas and onions) during two weeks on gene expression changes in normal colorectal mucosa of female sporadic adenoma patients and healthy controls was investigated by means of 
microarray analysis of 597 genes representing pathways relevant for carcinogenesis. The two study groups were chosen to investigate whether the effect of the intervention is different for patients compared to controls, as it is plausible that vegetables exert a different effect in persons who are at higher risk for the development of CRC. In total, comparison of pre- and post-intervention rectal biopsy samples revealed that 52 genes were differentially expressed, and according to literature review, 20 of these genes are likely to be related to (colon)carcinogenesis. In both patients and controls, seven genes were similarly modulated, for example fos proto-oncogene and ornithine decarboxylase. Thirteen genes were modulated differently in patients compared to controls, including cyclooxygenase-2 and mdm2-A in patients and cytochrome P450-27B1, $-2 \mathrm{C} 19,-2 \mathrm{D} 6$, $2 \mathrm{C} 9$, and $-3 \mathrm{~A} 4$ in controls. An increased intake of vegetables resulted in down-regulation of genes promoting cell proliferation and bioactivation of procarcinogens, and in upregulation of genes involved in cell growth arrest; in contrast, a decreased intake of vegetables resulted in down-regulation of genes inhibiting cell growth and up-regulation of genes promoting cellular dedifferentiation and bioactivation of procarcinogens. Furthermore, it seems that vegetables in patients affect genes involved in late stage of CRC, whereas in controls genes involved in the initiation phase are modulated. This is the first human study in which the effect of a vegetable intervention on gene expression changes at target level has been investigated. The results show that almost all the effects on modulating the expression of genes by altering vegetable intake can be mechanistically linked to cellular processes that explain either prevention of colorectal cancer risk by high vegetable intake or increased colorectal cancer risk by low vegetable intake.

To confirm the human findings and further elucidate mechanisms, mouse studies were performed using a comparable approach and technique. Although mice are often used to investigate the effect of diet on carcinogenesis ${ }^{7.10}$, the number of studies in which the effect on gene expression is studied is limited, is mostly investigated in combination with carcinogen administration and is restricted to analyses of a few genes. Therefore, the mouse studies carried out in this thesis provide much information for the effect of specific vegetable diets on the expression of multiple biologically relevant genes in target tissues. To achieve this, a mouse cDNA microarray was constructed, which contained the same genes as present on the microarray used for the human study.

To mimic the human dietary intervention study, the mouse study illustrated in Chapter 3 was performed in which the dose-dependent effect of a vegetable mixture was investigated on gene expression changes in colon mucosa. C57BL6 female mice were fed one of four different diets, containing no vegetables, $10 \%, 20 \%$ or $40 \%$ wt/wt vegetables mixture respectively, for a period of two weeks. The vegetables mixture consisted of the same combination and proportion of vegetables as used in the human intervention study, i.e. cauliflower (30\% wet wt), carrots (30\% wet wt), peas (30\% wet wt), and onions ( $10 \%$ wet wt). The results of this study show a dose-dependent effect of vegetables on gene 
expression, although in general this relationship is not linear. Almost all of the gene expression modulations occurred in the highest vegetable dose ( $40 \%)$ group. In total, the expressions of 39 genes were modulated, 17 of which are likely to play a role at different stages during $\mathrm{CRC}$ development, as indicated by literature review. Furthermore, for almost all these genes the altered expression can indeed explain reduced CRC risk. A diet high in vegetables positively modulated genes involved in different pathways, including inhibition of carcinogen formation, increasing DNA repair capacity, induction of apoptosis, and in reducing cell growth and tumor invasion. Many new genes were identified for which currently no modulation by vegetables has been reported, such as the detoxification gene ALDH1A1, several apoptosis genes (TNFRSF6, CASP3, -4 and -7, CTSB, TMSB10 and STAT1) and the tumor suppressor genes RRM1 and SLC26A3. Comparing the results of this mouse study with the results of the human study, only one gene is similarly affected by the vegetables in human and mouse colon, i.e. stearoyl-CoA desaturase (SCD). To date, two mouse (SCD1 and SCD2) genes and a single human SCD gene have been cloned and characterized. SCD is the rate-limiting enzyme in the cellular synthesis of monounsaturated fatty acids from saturated fatty acids. A proper ratio of saturated to unsaturated fatty acids contributes to membrane fluidity and cell-cell interaction. Abnormal alteration of this ratio has been shown to play a role in several physiological and disease states including diabetes, cardiovascular disease, obesity, hypertension, neurological disease, immune disorders, and cancer. However, causal relationships between SCD activity and these various disease states remain unclear. The activity of $\mathrm{SCD}$ is sensitive to for instance dietary changes, hormonal imbalance, temperature changes, alcohol and phenolic compounds. ${ }^{11}$ SCD activity was decreased in rat liver during starvation and diabetes and was rapidly induced to high levels upon refeeding high carbohydrate diets or upon insulin administration. ${ }^{12}$ The higher amount of carbohydrates present in the high vegetable diets could be responsible for the induction of SCD gene expression. The consequences of this on CRC risk are, however, indistinct. Although only one gene is similarly modulated in both studies, the results of both studies showed that a diet high in vegetables modulates genes in favor of CRC risk prevention via a number of similar pathways, i.e. inhibition of cell proliferation and detoxification of procarcinogens.

Despite the fact that gene expression studies are a fast and reproducible way to make an inventory of the effects of vegetables on gene expression changes, changes at the mRNA level are not necessarily proportional to the changes at the protein level because of differences in protein translation and degradation. Furthermore, proteins can undergo several functional posttranslational modifications like phosphorylation and glycosilation. Therefore, in addition to the gene expression analysis, a proteomic approach was undertaken to evaluate the effect of different doses of the vegetable mixtures on proteome changes in colon mucosa of female C57BL6 mice (Chapter 4). Differential protein expression was determined with $2 \mathrm{D}$ gel electrophoresis. Thirty proteins were found to be 
differentially expressed, and six proteins could be identified by mass spectrometry, namely MLRN, CAH-1, HMG-1, PAP3, GAPDH and OSCP. Except for OSCP, alterations in the levels of these proteins coincide with a pivotal role in the protection against colon cancer. The proteins are involved in different processes like cell growth, cell differentiation and apoptosis.

Comparing the results from the proteomic study with the gene expression study, no correspondence was observed between the human gene colon study and the mouse proteomic colon study. Only GAPDH was found to be differentially expressed in both the mouse proteomic study as in the mouse gene expression study, although dissimilar. Protein analysis by $2 \mathrm{D}$-gelelctrophoresis indicated a decreased protein expression in all diets compared to control, although this was not statistically significant. The immunoblotting results on the other hand showed a large variation between the various sub-pool samples. The observed difference between the mRNA levels and protein levels may be due to different subforms of GAPDH. To exert the wide variety of GAPDH functions, the different subforms of the protein need to be distinguished by the cell. This might be regulated by alternate splicing or posttranslational modification and results in protein products that differ in isoelectric point $(\mathrm{pI})$ and/or molecular weight. This might also explain the difference in protein expression as observed from the 2D-gelelectrophoresis and the immunoblotting experiments. ${ }^{13},{ }^{14}$ Considering the role of GAPDH in DNA replication, DNA repair and apoptosis, GAPDH might be an interesting candidate for protection against colon cancer. ${ }^{14}$ However, this has to be further investigated. The cDNAs representing the mRNAs coding for the other proteins found in the proteomic study were not present on the microarray used in the gene expression studies. However, besides GAPDH, for none of the other genes present on the array, differentially expressed proteins were identified. It could be that the gene expression difference resulted in a protein difference too small to be identified in the proteomics analysis. Despite this, in addition to the gene expression results, the results of the proteomic study also indicate modulation of proteins in favor of prevention of CRC risk.

Next to the amount of vegetables, the type of vegetables can be of importance for inducing particular gene expression changes. Therefore, the study described in Chapter $\mathbf{5}$ was carried out in which the effect of four individual vegetables, i.e. cauliflower, carrots, peas and onions, on the expression of genes in colon mucosa of female C57BL6 mice was examined. In total, 18 genes were differentially expressed by one or more of the specific vegetables: cauliflower was able to modulate most genes (10), closely followed by the onions (7) and carrots (7); least gene expression modulations occurred in the peas group (3). No genes were modulated solely by the carrots. According to literature review, ten of the 18 modulated genes are likely to be involved in CRC development, and seven out of these 10 are modulated in favor of CRC prevention. The four individual vegetables have about the same potential in modulating genes in support of lower CRC risk, although 
mostly via different mechanisms. Genes involved in cell growth regulation and maintaining homeostasis were affected in all vegetable groups. Furthermore, cauliflower, carrots and peas affected genes involved in induction of apoptosis; onions modulated genes which could play a role in maintaining DNA stability and induction of cellular differentiation; and peas, in addition to increasing the expression of an apoptosis gene, induced an angiogenesis inhibiting gene. From epidemiological studies it is known that with respect to prevention of CRC especially cruciferous and green leafy vegetables play a role. ${ }^{1}$ Indeed, cruciferous vegetables were able to induce most gene expression differences; however, most of these genes are currently not known to be involved in CRC protective mechanisms. The contribution of carrots, peas and onions in prevention of CRC risk could be of greater importance than until now considered.

In addition to this evaluation of vegetable specific gene modulations, a comparison can be made with the results of the studies described in Chapters 2 and 3. First, an evaluation will be made with the mouse vegetable mixture study described in Chapter 3 . The amount of the individual vegetables is equal to the amount of these specific vegetables in the highest vegetable mixture group $(40 \%)$ and therefore the contribution of the four individual vegetables to the combined effect of these vegetables on gene expression can be investigated. Eleven similar genes were modulated in both the vegetable mixture study and the individual vegetable study and for seven of them the modulation by the mixture could be explained by the effect of a particular vegetable. ACTB and CASP4 were modulated in the cauliflower group; OAT, EFHU1 and PMP22 were affected by cauliflower and carrots; and SCD2 and HIF1A were modulated by the onions. Although most of these genes (i.e. $\mathrm{ACTB}, \mathrm{EFHU1,}$ PMP22 and SCD2) are at this time not known to be involved in CRC protective mechanisms, the modulation in both vegetables studies gives a strong indication of their importance. Their role, however, in CRC development has to be further investigated. Comparing the results of the individual vegetables study with the human study illustrated in Chapter 2, two genes were similarly affected, i.e. ODC and, again, SCD. The role of SCD has already been discussed. The fact that modulation of this gene was observed in three independent vegetable studies and that the modulation is found in mice as well as in humans, indicates a strong regulation by vegetables and makes this gene of particular interest for further research. In addition to SCD, ODC was similarly modulated in the human study compared to the mouse individual vegetable study. This gene was downregulated in the high vegetable diet groups in both patients and controls, and in the mouse cauliflower and carrots group. ODC catalyzes the first step in the polyamine biosynthetic pathway, a highly regulated pathway associated with rapid growth states, including tumorigenesis. The polyamines are ubiquitous cellular polycations essential for optimal rates of cell growth and-differentiation. Increases in intracellular polyamine levels are related to increased cell proliferation and higher CRC risk. ${ }^{15,16}$ In normal colonic epithelium, polyamine levels are maintained at low levels, coordinated by the tumor 
suppressor gene APC. APC mediates ODC expression via a C-MYC dependent mechanism affecting ODC transcription. Wild-type APC suppresses the level of $\beta$-catenin, thereby reducing the formation of a complex between $\beta$-catenin and the lymphoidenhancing factor (LEF)/T-cell factor (Tcf) resulting in reduced expression of target genes like C-MYC and subsequent C-MYC target genes, like ODC. ${ }^{16}$ Inhibition of ODC by vegetable components has already been reported for $\beta$-carotene ${ }^{17}$, present in carrots, and indole-3-carbinol ${ }^{18}$, present in cauliflower. The results of the studies described in this thesis (Chapter 2 and 4 ) are in line with these previous investigations and provide more evidence of regulation of ODC expression by vegetables.

The polyamine biosynthetic pathway plays an important role in cell growth and differentiation in the intestine. Two other genes involved in this pathway were modulated in the vegetable mixtures study (Chapter 3), i.e. OAT and SAT; OAT was also affected in the cauliflower and carrots group (Chapter 5). OAT catalyzes the conversion of ornithine, which is the substrate for ODC, to glutamate semialdehyde, thereby reducing intracellular ornithine contents. ${ }^{19}$ Both in the highest vegetable mixture group $(40 \%)$ as in the cauliflower and carrots group, the expression of OAT was downregulated, which could result in an increased ornithine available for ODC, leading to higher polyamine levels. However, SAT, a key gene involved in polyamine catabolism, was upregulated in the highest vegetable group $(40 \%)$. This gene encodes for the key enzyme catalyzing the formation of shorther-chain amines from longer-chain ones, thereby reducing polyamine contents. The expression of the SAT gene is regulated by the K-RAS gene, which is an important proto-oncogene involved in development of CRC. Mutated K-RAS leads to a suppression of the nuclear hormone receptor peroxisomal proliferators-activated receptor $\gamma$ (PPAR $\gamma$ ), which in turn leads to a decreased SAT expression. ${ }^{16}$ The modulation of ODC, OAT and SAT by the vegetables provides evidence for vegetables to exert part of their beneficial effects in CRC through affecting the polyamine biosynthetic pathway, leading to reduced cell proliferation.

In addition to prevention of CRC, epidemiological studies provide evidence for protection against lung cancer incidence by vegetables and fruits. Particularly dark green and yellow-orange vegetables, rich in $\beta$-carotene and vitamin $\mathrm{E}$, are the specific types of vegetables, which best protect against lung cancer. In contrast to the colon and rectum, the lungs are not a site of direct contact, i.e. the vegetables pass the gastrointestinal tract but not the lungs as they are ingested. Furthermore, the dietary compounds that reach the lung may have been modulated by the first-pass metabolism in the liver. Therefore, the lungs are subject to only systemic exposure of vegetables-derived compounds and the mechanisms by which vegetables protect against lung cancer could therefore be different compared to that in the colon. To investigate the effect of vegetables on gene expression in the lung, the lungs of the mice used in the studies described in Chapters $\mathbf{3}$ and $\mathbf{5}$, were analyzed for gene expression changes (Chapter 6). In the vegetable mixture groups, 18 
genes were differentially expressed. Similar as in the colon study (Chapter 3), most gene expression differences occurred between the highest vegetable dose group $(40 \%)$ and one of the other diet groups. However, only seven of the 18 genes are, according to literature review, likely to be involved in mechanisms underlying lung cancer risk prevention. But, the hypothesized effects of gene expression modulation by the vegetable mixture are not always in favor of lung cancer risk prevention. The effects on IGFBP3 $(\downarrow)$ ), TGM2 $(\downarrow)$, TOP2A ( $\uparrow$, GLUL $(\downarrow)$ and CTSS $(\downarrow)$ are expected to enhance lung cancer risk, whereas the effects on GAPDH $(\uparrow)$ and FCER1G $(\uparrow)$ are expected to result in lung cancer risk prevention. In the individual vegetable groups, 11 genes were modulated, eight of which might be implicated in lung cancer preventive mechanisms, as indicated by literature review. Most gene modulations (6) took place in the carrots group, followed by peas (3) and cauliflower (2). Onions, together with carrots, were able to modulate the expression of only one gene, i.e. CTSS. The effect, however, on the expression of this gene is expected to increase lung cancer risk, by suppression of the immune system. Furthermore, also for the other modulated genes, the theoretical effects of the modulation by the individual vegetables on mechanisms underlying lung cancer prevention are not always beneficial. With respect to the effect of carrots on gene expression changes, five out of six modulated genes could be involved in lung cancer protective mechanisms; three (GLUL, SULT1A1 and SELENBP1) out of these five are affected in such a way that lung cancer protective mechanisms could be expected. For peas, two out of three modulated genes could be involved in lung cancer preventive pathways; only the effect on GSR could lead to lung cancer risk prevention by inducing the anti-oxidative defense. Finally, cauliflower was able to modulate the expression of two genes, i.e. BNIP1 ( $\uparrow$ ) and G1P2 ( $\downarrow$ ). Only BNIP1 is likely to be involved in lung cancer preventive pathways. Upregulation of BNIP1 by cauliflower could lead to increased apoptosis, which is generally regarded as a protective mechanism against cancer by removing genetically damaged cells before they can undergo clonal expansion. Taken all this together, vegetables are able to modulate genes involved in lung cancer preventive pathways, although, the modulation is not unambiguous in favor of reduced lung cancer risk. It is difficult, however, to predict the contribution of each gene modulation in the cancer process. The net effect of the gene expression modulation by the vegetables could still result in lung cancer risk prevention. Furthermore, genes which were not present on the microarray could play a role in lung carcinogenesis. More research is needed to investigate the effect of vegetables on gene expression changes in the lungs. Very interestingly, carrots were able to modulate most gene expression changes. Taken into account that it is known from epidemiological studies that in particular consumption of high amounts of carrots are able to protect against lung cancer, the results of the lung study provide rationale that indeed carrots are of importance in modulating this process.

Comparing the gene expression modulation occurring in colon with those in lung, corresponding and different genes were affected. Genes which are modulated in both 
tissues provide evidence for similar mechanisms by which vegetables protect against both types of cancer. When comparing the mouse lung study with the mouse colon study (Chapter 3, 4 and 5), eight genes were modulated in both the mouse colon and lung and include EFHU1, G1P2, GAPDH, SULT1A1, SEPP1, HPGD, TOP2A and BNIP1, however, the modulation was opposite (except for GAPDH). As already mentioned, the dietary compounds that reach the lung may have been modulated by the first-pass metabolism in the liver and these metabolites could be responsible for the observed effects. No (other) explanation for this remarkable result can be given and this has to be further investigated. The role of EFHU1, G1P2, and SEPP1 in carcinogenesis is currently not known. GAPDH (also identified in the colon proteomic study as differentially expressed, Chapter 4) and BNIP1 are involved in apoptosis, TOP2A plays a role in DNA replication, and SULT1A1 and HPGD are involved in biotransformation. In addition to modulating of similar genes, genes were affected by vegetables in either colon or lung. The number of genes affected in lung tissue is smaller and no genes are overrepresented in mechanisms of cancer prevention. However, remarkably, carrots seem to have the greatest potential of modulating genes in the lung, whereas in colon the individual vegetables appear to have equal promise for modulating genes in favor of CRC prevention.

Overall, the studies presented in this thesis provide much information on vegetable induced gene expression changes in target tissue where cancer risk is reduced by increased vegetable intake. In particular, the studies regarding vegetable induced gene expression changes in the colon and rectum support the hypothesis investigated in this thesis. In colon, vegetables modulated a lot of genes involved in biological and genetic pathways in favor of prevention of CRC risk. Genes were modulated for which already a relationship with vegetables and/or cancer prevention has been reported. In this regard, the studies provide new evidence which strengthens this relationship. In addition to these 'known' genes, new genes were identified for which, until now, no modulation by vegetables has been described, and/or no link is known with cancer. Remarkably, the dose of vegetables necessary to exert a measurable effect on the expression of genes in favor of cancer preventive mechanisms is relatively high in mice. The evidence regarding lung cancer risk prevention by vegetable induced gene expression changes is less clear compared to the results of the colon studies. The role of carrots is of particular interest and most promising for modulating genes in support of reduced lung cancer risk. The results of the lung studies, however, provide too little information to either reject or confirm the hypothesis. Further research is needed in which the contribution of specific genes and pathways modulated by different vegetables types is investigated. Several genes are of particular interest for future studies. Especially $\mathrm{SCD}$ is of interest because the modulation by vegetables was observed in three independent vegetable studies in mice as well as in humans. In addition, the role of COX-2 mediated gene expression by vegetables is very 
relevant, because of its established role in CRC risk. In addition to specific genes, several pathways are of particular interest. In both human and mouse studies, mostly genes involved in inhibition of cell growth are modulated by the vegetables. The affected pathways predominantly comprise the polyamine biosynthetic pathway and the apoptosis pathway. By using knockout and/or transgenic systems, the effect of vegetables on the contribution of the absence or presence of particular genes in the cancer process can be examined. Relatively new technologies like RNA interference and laser capture microdissection provide new possibilities in optimizing gene expression research. Furthermore, to investigate the effect of vegetables on gene expression changes in different stages of the carcinogenic process, studies in which vegetable are applied before and/or after administration of carcinogens can be carried out. In addition to dose-response studies, time-line studies could provide information about specific time-points and duration of vegetable induced gene expression effects. The results of these studies will provide a more complete and fundamental understanding of cancer aetiology, which is necessary for the development of improved prevention strategies based on dietary advice. Improved and more convincing evidence for the anticarcinogenic effects of vegetables may result in higher consumption of vegetables by the general public. 


\section{References}

1. World Cancer Research Fund. Food, Nutrition and the Prevention of Cancer a global perspective. Washington: BANTA Book group, 1997.

2. World Health Organization. World Cancer Report. Lyon: IARCPress, 2003.

3. Willett WC. Diet, nutrition, and avoidable cancer. Environ Health Perspect 1995;103 Suppl 8:165-70.

4. Fearon ER, Vogelstein B. A genetic model for colorectal tumorigenesis. Cell 1990;61:759-67.

5. Arends JW. Molecular interactions in the Vogelstein model of colorectal carcinoma. J Pathol 2000;190:412-6.

6. Chung DC. The genetic basis of colorectal cancer: insights into critical pathways of tumorigenesis. Gastroenterology 2000;119:854-65.

7. Kallay E, Adlercreuty H, Farhan H, Lechner D, Bajna E, Gerdenitsch W, Campbell M, Cross HS. Phytoestrogens Regulate Vitamin D Metabolism in the Mouse Colon: Relevance for Colon Tumor Prevention and Therapy. J Nutr 2002;132:3490S-3493S.

8. Katchamart S, Williams DE. Indole-3-carbinol modulation of hepatic monooxygenases CYP1A1, CYP1A2 and FMO1 in guinea pig, mouse and rabbit. Comp Biochem Physiol C Toxicol Pharmacol 2001;129:377-84.

9. van Kranen HJ, van Iersel PW, Rijnkels JM, Beems DB, Alink GM, van Kreijl CF. Effects of dietary fat and a vegetable-fruit mixture on the development of intestinal neoplasia in the ApeMin mouse. Carcinogenesis 1998;19:1597-601.

10. Kim DJ, Shin DH, Ahn B, Kang JS, Nam KT, Park CB, Kim CK, Hong JT, Kim YB, Yun YW, Jang DD, Yang KH. Chemoprevention of colon cancer by Korean food plant components. Mutat Res 2003;523-524:99-107.

11. Ntambi JM. Regulation of stearoyl-CoA desaturase by polyunsaturated fatty acids and cholesterol. J Lipid Res 1999;40:1549-58.

12. Oshino N, Sato R. The dietary control of the microsomal stearyl CoA desaturation enzyme system in rat liver. Arch Biochem Biophys 1972;149:369-77.

13. Mezquita J, Pau M, Mezquita C. Several novel transcripts of glyceraldehyde-3-phosphate dehydrogenase expressed in adult chicken testis. J Cell Biochem 1998;71:127-39.

14. Sirover MA. New insights into an old protein: the functional diversity of mammalian glyceraldehyde3-phosphate dehydrogenase. Biochim Biophys Acta 1999;1432:159-84.

15. Martinez ME, O'Brien TG, Fultz. KE, Babbar N, Yerushalmi H, Qu N, Guo Y, Boorman D, Einspahr J, Alberts DS, Gerner EW. Pronounced reduction in adenoma recurrence associated with aspirin use and a polymorphism in the ornithine decarboxylase gene. Proc Nati Acad Sci U S A 2003; 100:7859-64.

16. Babbar N, Gerner EW. Polyamines as modifiers of genetic risk factors in human intestinal cancers. Biochem Soc Trans 2003;31:388-92.

17. Phillips RW, Kikendall JW, Luk GD, Willis SM, Murphy JR, Maydonovitch C, Bowen PE, Stacewicz-Sapuntzakis M, Wong RK. beta-Carotene inhibits rectal mucosal ornithine decarboxylase activity in colon cancer parients. Cancer Res 1993;53:3723-5.

18. Shertzer HG, Senft AP. The micronutrient indole-3-carbinol: implications for disease and chemoprevention. Drug Metabol Drug Interact 2000;17:159-88.

19. Han X, Kazarinoff MN, Seiler N, Stanley BA. Rat colon ornithine and arginine metabolism: coordinated effects after proliferative stimuli. Am J Physiol Gastrointest Liver Physiol 2001;280:G389-99. 



\section{Nederlandse Samenvatting}

Voedselbereidingswijzen en de voedselsamenstelling zijn belangrijke exogene factoren die het risico op kanker in positieve of negatieve zin kunnen beinvloeden. De meest consistente bevinding betreffende de voeding als determinant van kankerrisico is de associatie tussen consumptie van groenten en fruit en een verlaagd risico op verscheidene soorten kanker. Overtuigend epidemiologisch bewijs is er voor preventie van kanker van het maagdarm stelsel (mondholte, keel, slokdarm, maag, dikke darm) en kanker van de luchtwegen (long). Wereldwijd is longkanker de meest voorkomende vorm van kanker, gevolgd door borstkanker en op de derde plaats door dikke darmkanker. De consumptie van een aanzienlijke hoeveelheid gevarieerde groenten per dag $(>400 \mathrm{~g})$ kan de incidentie van longkanker en dikke darmkanker verlagen met 45, respectievelijk 40 procent (range 3050). De manieren waarop groenten hun werking uitoefenen in het lichaam zijn gevarieerd. Uit de resultaten van experimentele studies blijkt dat groenten in staat zijn om specifieke biotransformatie enzymen in de cel te induceren dan wel te remmen, de cel te beschermen tegen oxidatieve stoffen en vrije radicalen, de inductie van geprogrammeerde celdood (apoptose) te stimuleren, enzovoorts. Deze processen gaan vaak gepaard met veranderingen in de expressie van genen. Echter, tot nu toe heeft het onderzoek hiemaar zich voornamelijk gericht op het meten van veranderingen in de expressie van biotransformatie genen in surrogaat weefsel zoals lymfocyten. Er zijn maar weinig studies gedaan waarin het effect van groenten of van bestanddelen van groenten op genexpressie veranderingen in doelorganen is onderzocht. Een nieuwe technologie die nu gebruikt kan worden om het effect te bepalen van een specifiek dieet op de expressie van talrijke genen of eiwitten in een enkel experiment is de microarray techniek. Deze methode kan helpen bij het identificeren van de mechanismen op het niveau van de genen die door groenten beinvloed worden waardoor de in vorige studies geobserveerde verlaging van het kankerrisico mechanistisch onderbouwd kan worden.

De hypothese die in dit proefschrift is onderzocht is dat een belangrijke bijdrage van de anticarcinogene effecten van groenten in de dikke darm en in de long wordt gevormd door het moduleren van de expressie van genen die betrokken zijn bij biologische en genetische processen relevant voor (chemische) carcinogenese. Het doel van dit proefschrift is om meer inzicht te verschaffen in de moleculaire mechanismen waardoor groenten hun anticarcinogene werking uitoefenen, door onderzoek te verrichten op het niveau van het genoom in de relevante doelcellen.

De opeenvolgende tumorstadia in de dikke darm worden beschreven in het Vogelstein model. Dit model beschrijft de ontwikkeling van dikke darmkanker door middel van een aantal opeenvolgende genetische veranderingen waarbij een aantal genen 
zijin betrokken zoals APC (adenopolyposis coli), K-RAS, DCC and p53. Gedurende de afgelopen tien jaar zijn er aanvullende genetische veranderingen en specifieke moleculaire processen geïdentificeerd. Het is duidelijk geworden dat de belangrijkste genen van het Vogelstein model, intact dan wel gemuteerd, interacteren en een netwerk vormen en daarbij andere genen beïnvloeden. Deze genetische processen en de betrokken genen zijn mogelijke moleculaire doelwitten voor de bescherming tegen het ontstaan van dikke darmkanker door groenten.

Het onderzoek beschreven in Hoofdstuk 2 is uitgevoerd om de genen te identificeren die in vivo gemoduleerd worden in humaan dikke darm weefsel door de inname van groenten te veranderen. In deze humane voedingsinterventie studie is het effect onderzocht van een halvering $(=75 \mathrm{~g} / \mathrm{dag})$ of verdubbeling $(=300 \mathrm{~g} / \mathrm{dag})$ van $\mathrm{de}$ mediane dagelijkse inname van groenten. Hiertoe consumeerden twintig vrouwelijke sporadische poliep patiënten en acht vrouwelijke gezonde vrijwilligers gedurende twee weken een mix van vier groenten (bloemkool, wortelen, erwten en uien) in plaats van de groenten die zij normaal gesproken zouden eten. Voor en na deze twee weken zijn stukjes darmweefsel geisoleerd en onderzocht op genexpressie veranderingen met behulp van microarray analyse van 597 genen die betrokken zijn bij processen relevant voor carcinogenese. De twee onderzoeksgroepen zijn gekozen om te bekijken of het effect van de interventie verschillend is voor personen die een hoger risico lopen om dikke darmkanker te ontwikkelen ten opzichte van gezonde personen. Uit het onderzoek blijkt dat 52 verschillende genen gemoduleerd werden door de groenten, en door middel van literatuur onderzoek zijn 20 van deze 52 genen geïdentificeerd waarvan aannemelijk is dat ze een rol spelen bij het proces van (dikke darm) kanker. Zeven van deze 20 genen werden in zowel patiënten als gezonde proefpersonen gemoduleerd, altijd in dezelfde richting, en dertien genen werden alleen gemoduleerd in patiènten of in gezonde proefpersonen. Een verhoogde inname van groenten resulteerde in een afname van de expressie van genen die celproliferatic stimuleren en pro-carcinogenen activeren, en in een verhoging van de expressie van genen betrokken bij het stoppen van celgroei. Daarentegen, een verlaagde inname van groenten resulteerde in een afname van de expressie van genen betrokken bij het remmen van de celgroei en in een toename van de expressie van genen die cellulaire dedifferentiatie bevorderen en pro-carcinogenen activeren. Deze resultaten laten zien dat bijna alle genexpressie veranderingen mechanistisch verbonden zijn met cellulaire processen die een afname van het risico op dikke darmkanker verklaren door een hoge groenten inname en een toename van het risico op dikke darmkanker verklaren door een lage groenten inname. Verder lijkt het dat groenten in patiënten genen beïnvloeden die betrokken zijn in de latere stadia van het ontstaan van dikke darmkanker, terwijl in gezonde proefpersonen genen worden gemoduleerd die een rol spelen in de initiatie fase. Deze humane studie is de eerste studie waarin het effect van consumptie van groenten is onderzocht op genexpressie veranderingen in het doelorgaan, namelijk de dikke darm. 
Om de bevindingen van de humane studie te bevestigen en mechanismen verder op te helderen zijn muizenstudies uitgevoend waarbij eenzelfde aanpak en technick is gebruikt. Hoewel muizen vaak gebruikt worden om her effect van een dieet op het ontwikkelingsproces van kanker te bestuderen, is het aantal studies waarin het effect op genexpressie is bestudeerd beperkt. Deze zijn meestal uitgevoerd in combinatie met toediening van een carcinogeen en beperkt tot het analyseren van een klein aantal genen. De muizenstudies die beschreven zijn in dit proefschrift geven daarom veel informatie over het effect van een specifiek groentedieet op de expressie van biologisch relevante genen in doelweefsel. Om dit te bewerkstelligen is een muizen cDNA microarray gemaakt die dezelfde genen bevat als de cDNA microarray voor de humane studie.

De muizenstudie beschreven in Hoofdstuk 3 is een nabootsing van de humane studie. In deze muizenstudie is de afhankelijkheid van de dosis van een mix van groenten op genexpressie veranderingen in de dikke darm van de muis bestudeerd. Hiervoor werden C57B16 vrouwelijke muizen verdeeld in vier groepen (7 muizen per groep) die elk gedurende twee weken een ander dieet kregen, namelijk een dieet zonder groenten, met $10 \%, 20 \%$ of met $40 \%$ van de groentenmix. De groentenmix bestond uit dezelfde combinatie en verhouding als de groentenmix gebruikt in de humane studic, namelijk bloemkool, wortelen, erwten en uien $(30 \%, 30 \%, 30 \%$ en $10 \%$ natgewicht, respectievelijk). De resultaten van deze studie laten een dosis afhankelijkheid zien van het effect van groenten op genexpressie, echter, deze relatie is over het algemeen niet lineair. Bijna alle genexpressie veranderingen vonden plaats in de hoogste dosis $(40 \%)$ grocp. In totaal werden er 39 genen gemoduleerd, waarvan er 17 een rol kunnen spelen in de ontwikkeling van dikke darmkanker, zoals beschreven in de literatuur. Het effect op 15 van deze 17 genen is ten gunste van een verlaging van het kankerrisico. Een dieet met een hoog gehalte aan groenten moduleert op een positieve manier de expressie van genen die betrokken zijn bij verschillende processen, zoals remming van de vorming van carcinogenen, toename van DNA herstel capaciteit, inductie van apoptose, en in een afname van celgroei en tumor invasie. Verschillende nieuwe genen zijn geidentificeerd waarvoor tot nu toe nog geen modulatie door groenten werd beschreven. Slechts één gen wordt op gelijke wijze gemoduleerd in zowel de humane groenten studie als in de muizen groenten studie, namelijk stearoyl-CoA desaturase (SCD). Echter, beide studies laten zien dat een dieet met een hoge inname van groenten genen moduleert in het voordeel van een afname van het dikke darmkanker risico via een aantal dezelfde processen, namelijk remming van celproliferatie en detoxificatie van pro-carcinogenen.

Vervolgens is met behulp van de proteomics benadering het effect van verschillende doses van de groentenmix op eiwit veranderingen in de dikke darm van dezelfde muizen onderzocht. (Hoofdstuk 4) Door middel van 2D gel electroforese is de expressie van eiwitten bepaald. Dertig eiwitten kwamen verschillend tot expressie tussen de verschillende dieetgroepen, en zes daarvan konden geïdentificeerd worden met massa 
spectrometrie (MLRN, CAH-1, HMG-1, PAP3, GAPDH en OSCP). Met uitzondering van OSCP zijn de expressieveranderingen van deze eiwitten in overeenstemming met een lager risico op het ontstaan van dikke darmkanker bij een hogere groenten inname. De eiwitten zijn betrokken bij verschillende processen zoals celgroei, celdifferentiatie en apoptose. Alleen GAPDH is geïdentificeerd in zowel de eiwitexpressie studie in muizen als de genexpressie studie in muizen, echter de waargenomen modulatierichting is tegengesteld. Geen overeenkomsten werden gevonden tussen de eiwitexpressie studie in muizen en de genexpressie studie in proefpersonen.

Naast de hoeveelheid groenten is ook onderzocht of het soort groenten van belang is voor het induceren van genexpressie veranderingen. De studie beschreven in Hoofdstuk $\mathbf{5}$ is uitgevoerd om het effect van vier individuele groenten, namelijk bloemkool, wortelen, erwten en uien, op de expressie van genen in het dikke darmweefsel van vrouwelijke C57BL.6 muizen te onderzoeken. In deze studie werden in totaal 18 genen gemoduleerd door één of meerdere groenten. Bloemkool moduleerde het grootste aantal genen (10), gevolgd door de uien (7) en de wortelen (7), erwten induceerden de minste genexpressie veranderingen (3). Met behulp van literatuur gegevens kan onderbouwd worden dat tien van de 18 genen waarschijnlijk een rol spelen in de ontwikkeling van dikke darmkanker. Zeven van deze tien genen worden op een dusdanige manier gemoduleerd dat een verlaging van het kankerrisico te verwachten is. De potentie van de individuele groenten om genen te moduleren in overeenstemming met een afname van het kankerrisico lijkt gelijk, hoewel de betrokken processen meestal verschillend zijn. Genen betrokken bij de regulatie van celgroei en het handhaven van homeostase werden door alle vier de groenten beinvloed. Bloemkool, wortelen en erwten moduleerden genen betrokken bij apoptose; genen gemoduleerd door uien spelen een rol bij het behouden van DNA stabiliteit en inductie van celdifferentiatie; en erwten, naast het verhogen van de expressie van een apoptose gen, induceerden de expressie van een angiogenese remmend gen. Hoewel bloemkool de meeste genen moduleerde, is van het merendeel van deze genen op dit moment niet bekend of ze betrokken zijn bij kankerbeschermende processen.

De resultaten van de studie met individuele groenten in muizen kunnen vergeleken worden met de humane interventie studie (Hoofdstuk 2) en de muizenstudie met groentenmix (Hoofdstuk 3). De hoeveelheid van de groenten in de groep die de hoogste dosis $(40 \%)$ van de groentenmix kreeg is gelijk aan de som van de individuele groenten. Hierdoor kan de bijdrage van de vier individuele groenten aan het gecombineerde effect van deze groentenmix bestudeerd worden. Elf genen werden in beide studies gemoduleerd en voor zeven hiervan kan de modulatie in de studie met groentenmix verklaard worden door het effect van een specifieke groente. Hoewel van de meeste van deze genen op dit moment niet bekend is of ze een rol spelen in preventieve processen tegen darmkanker, geeft de modulatic van deze genen door groenten in beide studies het belang van deze genen aan. Hun mogelijke rol in de ontwikkeling van dikke darmkanker moet echter verder 
onderzocht worden. Twee genen werden gemoduleerd in zowel de humane interventie studie (Hoofdstuk 2) als in de studie met individuele groenten in muizen, namelijk ODC en SCD. De expressie van ODC was verlaagd in de groep die de hoogste dosering aan groenten ontving, in zowel patiènten als controles, en in de muizen die het bloemkool dieet of wortelen dieet kregen.

Naast preventie van dikke darmkanker is er vanuit epidemiologische studies bewijs voor een bescherming tegen longkanker door groenten en fruit. In het bijzonder zijn de donkergroene en de oranje-rode groenten, rijk aan $\beta$-caroteen en vitamine E, de groenten waarvoor het bewijs het meest overtuigend is. In tegenstelling tot de darm, zijn de longen geen orgaan waarmee de groenten na inname in direct contact komen. Daarom zijn de longen alleen onderhevig aan systemische blootstelling van groenten bestanddelen. Bovendien kunnen de groenten componenten die de longen bereiken vooraf gemetaboliseerd worden in de lever. De mechanismen waardoor groenten beschermen tegen de vorming van longkanker kunnen daarom anders zijn vergeleken met de dikke darm. Om het effect van groenten op genexpressie veranderingen in de long te onderzoeken, zijn de longen van de muizen uit de studies beschreven in de Hoofdstukken 3 en 5 geanalyseerd (Hoofdstuk 6). In de longen van de muizen uit de studie met groentenmix werden 18 genen gemoduleerd. Net als in de studie waarin het effect in de dikke darm is onderzocht (Hoofdstuk 3), traden de meeste genexpressie verschillen in de longen op tussen de groep muizen die de hoogste dosering groenten ontvingen en één van de andere groepen. Echter, slechts zeven van de 18 genen zijn waarschijnlijk betrokken bij processen die beschermen tegen longkanker (literatuuronderzoek), waarbij vervolgens de richting van modulatie niet altijd in het voordeel is van preventie van longkankerrisico. In de longen van de muizen in de studie met individuele groenten werden in totaal 11 genen gemoduleerd, waarvan er acht, zoals gevonden in literatuuronderzoek, een rol kunnen spelen in mechanismen die beschermen tegen longkanker. De meeste (6) genmodulaties vonden plaats in de groep die wortelen consumeerde. In de groep die erwten kreeg toegediend traden drie genmodulaties op en in de groep die bloemkool at twee genmodulaties. Uien waren in staat om slechts één gen te moduleren, namelijk CTSS. Echter, het verwachte effect op het risico op longkanker door de modulatie van dit gen door de groenten is verhogend, door onderdrukking van het immuunsysteem. Ook voor de andere genen die gemoduleerd worden door de individuele groenten is het te verwachten effect op processen die beschermen tegen longkanker niet altijd positief, zoals beschreven in de literatuur. De resultaten van dit onderzoek laten zien dat groenten in staat zijn genen te moduleren die betrokken zijn bij processen die beschermen tegen longkanker, maar dat de modulatie niet altijd in het voordeel lijkt van een afname van het longkanker risico. Interessant is echter dat de wortelen de meeste genexpressie veranderingen induceren. Het is vanuit epidemiologisch onderzoek bekend dat een hoge inname van wortelen geassocieerd is met een afname van het longkanker risico. De resultaten van de 
studie met individuele groenten in muizen bevestigen dat de consumptie van wortelen van belang zijn bij het moduleren van het longkanker proces. Bij het vergelijken van de genmodulaties in de long studie met die van de dikke darm studies (Hoofdstuk 3, 4 en 5) zijn er acht genen die in beide studies gemoduleerd worden, nameliik EFHU1, G1P2, GAPDH, SULT1A1, SEPP1, HPGD, TOP2A en BNIP1. Echter, de modulatie was tegengesteld (behalve voor GAPDH). Naast overeenkomstige genen werden ook verschillende genen gemoduleerd in beide organen. Het aantal genen gemoduleerd in de long is kleiner en ook zijn deze genen niet in duidelijke mate betrokken bij kankerbeschermende mechanismen. Opvallend is wel dat in de long de wortelen de grootste potentie hebben om genen te moduleren, terwijl in de dikke darm de individuele groenten eenzelfde potentie hebben om genen te moduleren in het voordeel van dikke darmkanker preventic.

Over het geheel genomen verschaffen de onderzoeken beschreven in dit proefschrift veel informatie over het effect van groenten op de veranderingen in genexpressie in doelorganen waar het kankerrisico wordt verlaagd door een verhoogde inname van groenten. In het bijzonder ondersteunen de studies betreffende de genexpressie veranderingen in de dikke darm de hypothese die in dit proefschrift is onderzocht. In de dikke darm moduleren de groenten veel genen die betrokken zijn bij biologische en genetische processen leidend tot preventie van dikke darmkankerrisico. Genen waarvoor al een relatie met groenten en/of kanker bekend is werden gemoduleerd, maar ook 'nieuwe' genen werden beïnloed waarvoor deze relatie nog niet bekend is. Opvallend is dat de dosis groenten die nodig is om genen te moduleren in het voordeel van een afname van het kankerrisico relatief hoog is in muizen. Dit ondersteunt de aanbeveling dat mensen veel groenten moeten consumeren om het risico op kanker te verlagen. Het bewijs voor modulatic van genen in beschermende processen tegen longkanker zijn minder duidelijk vergeleken bij de resultaten uit de dikke darm studies. Bijzonder interessant en veelbelovend is echter de rol van de wortelen in het moduleren van genen in het voordeel van een afname van het longkanker risico. De resultaten van de longstudie voorzien echter in te weinig informatie om de hypothese te behouden of te verwerpen. Meer ondetzoek is nodig waarin de bijdrage van specifieke genen en processen door verschillende groenten wordt onderzocht.

Verscheidene genen zijn interessant voor vervolgonderzoek. In het bijzonder SCD, omdat de modulatie van dit gen in drie onafhankelijke studies in zowel de mens als de muis is waargenomen. Daarnaast is de modulatie van COX-2 door de groenten in de humane studie zeer belangrijk, vanwege de rol van COX-2 in dikke darmkanker. Behalve specifieke genen, zijn ook specifieke processen van bijzondere interesse. In zowel de humane als de muizenstudies zijn genen gemoduleerd die een rol spelen bij remming van de celgroei. Betrokken processen die hierbij de overhand hebben zijn het polyamine 
biosynthese proces en apoptose. Door gebruik te maken van genetisch gemodificeerde muizen kan het effect van groenten en de bijdrage van een specifiek gen op verschillende processen bestudeerd worden. Daarnaast kunnen relatief nieuwe technieken zoals RNA interference en laser capture microdissectic een bijdrage leveren aan het optimaliseren van genexpressie onderzoek. De resultaten verkregen in de studies in dit proefschrift geven nieuwe aanknopingspunten voor vervolgonderzoek en kunnen leiden tot de ontwikkeling van functionele voeding en/of voedingssupplementen die beter beschermen tegen het ontstaan van kanker. 



\section{Dankwoord}

Opeens is het dan toch zover. Mijn boekje is af, alleen nog 'effe' het dankwoord schrijven. Ik kan me nog heel goed herinneren dat ik viereneenhalf jaar geleden begon als aio, en dat ik toen geen idee had wat me nu eigenlijk precies te wachten stond. Het is een ontzettende boeiende en leerzame periode geweest, zowel op wetenschappelijk als persoonlijk gebied. Ondanks alle moeilijke momenten zou ik het zo weer doenl Maar ik besef ook heel goed dat dit boekje niet tot stand zou zijn gekomen zonder de hulp van vele anderen: allemaal heel hartelijk bedankt voor jullie bijdrage aan mijn boekjell!

Als eerste wil ik mijn promotor, prof. Jos Kleinjans, en co-promotor, dr. Joost van Delft, bedanken voor hun vertrouwen dat zij altijd in mij hebben gehad. Als eerste 'genomics' aio weet ik dat het geen gemakkelijke klus is geweest om mij te begeleiden. Iedere stap was voor ons allemaal nieuw en op onbekend terrein dat nog helemaal ontgonnen moest worden. Jos, ik kan me nog goed jouw woorden herinneren toen we voor de zoveelste keer over de resultaten van de humane studie aan het brainstormen waren: 'g...verdomme, ik heb nog nooit zolang en zovaak over dezelfde resultaten zitten discussiëren'. Maar het heeft zijn vruchten afgeworpen! Vooral in de laatste fase van mijn aio-periode heb jii een belangrijke rol gespeeld. Je stelde me driekwart jaar geleden voor een -voor mij op dat moment ondenkbaar haalbare- deadline: 1 augustus moet het af! En met jouw strikte begeleiding is het gelukt! Heel erg bedankt. Joost, als bedenker van dit project en mijn directe begeleider wil ik je bedanken dat je me de kans en vrijheid hebt gegeven om mijn eigen invulling eraan te geven. En toch hield je alles wat ik deed in de gaten en gaf je op gezette tijden feedback. Leuk dat we de komende tijd weer samenwerken.

Alle collega's van de capaciteitsgroep GRAT wil ik bedanken voor hun hulp en betrokkenheid bij mijn onderzoek en voor alle gezelligheid! Sumero, als analist was jii verbonden met dit aio-project en je hebt me wegwijs gemaakt in de wereld van RNA en array. Je hebt me bij zowat alles geholpen waar het maar kon: RNA isolatic, het ontwikkelen van de array methode, het 'groenten rijden', het bereiden van de groenten diëten voor de muisjes en zo kan ik nog wel even doorgaan. Maar ook kon ik altijd bij je terecht als ik het niet meer zag zitten en je liet me dan 'lekker zeuren', Jij bent niet voor niets één van mijn paranimfen! Ik wil je hier heel erg voor bedanken! Veel succes en plezier in de nieuwe fase van je leven na het stoppen met werken bij GRAT. Daniëlle, helaas alweer een tijdje ex-collega want iij werkt nu 'op vijf, bedankt voor de gezellige studietijd en de samenwerking bij-inderdaad- 'ons' nitraat-diabetes onderzoek. En ook nog samen aio geweest bij GRAT! Ik zal de tijd die we hebben doorgebracht gedurende de POTcursussen (met name de eerste) niet snel vergeten. Leuk dat we ook nu nog cen beetje bij elkaar in de buurt blijven. Edwin, bedankt voor het meten van alle plasma samples en voor 
het invallen tijdens het 'groenten rijden'. Jan D. en Pascal, bedankt voor alle hulp als mijn I-Mac respectievelijk PC weer eens vreemd deed. En Pascal, ook bedankt voor het organiseren van alle gezellige en vooral niet competitieve bowling-avondjes. Alle aio's van GRAT bedankt voor de leuke tijd en alle aio-etentjes. Lonneke, met jou samen op congres in Pisa, die chocolade taart ging er wel in! Anne, de ontelbare per's en daaropvolgende gel's die je tijdens je stage periode hebt gedaan waardeer ik enorm. Zonder jou was de muizenarray nooit zo snel een feit geworden. Leuk dat je binnenkort naar 'het zuiden' komt om hier je aio-project af te ronden. Yvonne en Sabine, nog niet zo heel lang mijn kamergenootjes, maar het is me tot nu toe heel goed bevallen. Wil graag op deze aio-kamer blijven zitten als ik dadelijk post-doc ben. Githa, bedankt voor je bijdrage aan hoofdstuk 4 . Zonder jouw toewijding aan de ontelbare eiwitspots was dit hoofdstuk er nooit gekomen. Heel veel succes bij het promoveren!

De studie beschreven in hoofdstuk 2 had niet tot stand kunnen komen zonder de inzet van dr. Leopold Engels, gastroenteroloog in het Maasland ziekenhuis te Sittard. Ik weet dat ik u soms tot wanhoop heb gedreven met de vraag of er misschien nog nieuwe 'controle' personen waren die mee wilden doen met het onderzoek. Bedankt voor alle tijd en energie die $u$ hebt besteed aan de werving van geschikte proefpersonen, de extra endoscopieën en de klinische informatic voor het artikel. Ook het verpleegkundig personeel en de rwee secretaresses van de coloscopie afdeling ben ik heel dankbaar voor hun fantastische hulp en betrokkenheid bij het onderzoek en mijn persoonlijke leven. In het bijzonder wil ik Tonny bedanken voor het uitzoeken en benaderen van de geschikte polieppatiënten, en Carla als contactpersoon in de laatste fase van het onderzoek. Alle proefpersonen ook heel hartelijk bedankt voor jullie deelname (iedere dag diezelfde groenten gedurende twee weken, en dan ook nog als 'beloning' een extra endoscopie)!

Rob en Erika van het Genoomcentrum bedankt voor jullie hulp bij het spotten van de muizen-arrays en alle andere RNA dingerjes. Bianca, als collega-genomics aio van de capgroep Populatie Genetica heb ik je beter leren kennen. Maar ik kan zeggen dat je daarnaast ook steeds meer een vriendin bent geworden waarmee ik naast alle array-troubles ook persoonlijke dingen kan bespreken. Gaan we nou ook eens iets anders leuks doen dan alleen maar lunchen? En succes met die allerlaatste zware loodjes!

CPV, bedankt voor het verzorgen van mijn muisjes!

Next, 1 would like to thank Suzy and Tomasz from the Center for Statistics, Limburg University Centre, Diepenbeek (Belgium) for the statistical analyses of the innumerable mouse-array data. In your sparse time besides your 'normal' work you managed to do all the analyses in a very short time. Thanks so very much.

Gelukkig is er ook nog een leven naast de wetenschap. Petra, al van kleins af aan vriendin met jou. De laatste paar jaar hebben we nog beter contact en je hebt me na het overlijden van mijn moeder op sleeptouw genomen. Door jou heb ik er nog twee vriendinnen en een vriend bij gekregen: Xiomara (ja, ik kom nu echt heel snel naar 
Curaçaoll! Kun je me alle mooie duikplekjes laten zien) en Daniëla \& Patrick. Danièla \& Patrick, jullic nuchtere maar wel zeer inleefbare mening hebben mij vaak weer met twee benen op de grond gezet. Mandy, bedankt voor alle gezellige sport - alias bijkletsavondjes. En kop op, nog even doorzetten en je hebt je diplomal Steffie, mijn hartsvriendin op de middelbare school! Ben blij dat je je plekje hebt gevonden in Utrecht en dat je ondanks de afstand toch betrokken bent gebleven bij mijn leven. We moeten snel weer een weekendje plannen!

Bettina, al meer dan tien jaar de vriendin van mijn broer, maar ik ben trots om te kunnen zeggen je naast mijn 'schone zusje' ook éen van mijn allerbeste vriendinnen bent. Vooral de wandelingen op de Brunssumerheide zijn een zeer welkome afleiding geweest in de drukste tijd van mijn aio-periode. Ik kon altijd mijn hoofd leegmaken, want jij wees me de weg. Heel fijn dat je straks ook letterlijk achter me wilt staan als paranimf!

Mijn familie, en in het bijzonder mijn opa en oma, heel erg bedankt dat jullie altijd betrokken zijn geweest bij waar ik mee bezig was, al hebben jullie volgens mij nog steeds geen idee wat dat nu was. Ik hoop dat dit met dit boekje en de promotie wat duidelijker is geworden. Opa en oma, de sterkste schakel in de familie, bedankt voor jullie steun en interesse en ik kom snel weer een keertie eten.

Papa en mama en Ralph, samen met jullie heb ik het grootste gedeelte van mijn leven tot nu doorgebracht. De klap kwam dan ook zeer hard aan toen mama drie en een half jaar geleden kwam te overlijden. Des te meer voel ik vanaf dat moment hoe belangrijk jullie allemaal zijn in mijn leven en hoe dankbaar ik voor alles ben wat jullie me hebben gegeven en nog steeds geven. Ralph, alias knullie, mijn grote broer waarbij ik altijd weer klein kan zijn. De band die wij hebben is heel bijzonder en fijn dat ik altijd bij je terecht kan. Papa, mijn trotse vader, onze band wordt alleen maar sterker en ik ben heel bij dat je in Marjo een geweldige partner hebt gevonden waarmee je hopelijk nog heel lang gelukkig bent. En lieve mama, hoe moeilijk het ook is om zonder jou te leven, ik weet en voel dat je erbij bent.

\section{Liefs Simone}




\section{Curriculum Vitae}

Simone Gerty Jeannette van Breda werd geboren op 2 januari 1977 te Hulsberg. Na het behalen van het Atheneum bèta diploma aan het Sintermeerten College te Heerlen in juni 1995, heeft ze enkele maanden geneeskunde gestudeerd aan de Katholieke Universiteit Leuven in België. In december 1995 begon zij haar studie Gezondheidswetenschappen met als afstudeerrichting Milieugezondheidkunde aan de Universiteit Maastricht. Tijdens deze studie werkte zij als student-onderzoeker bij de capaciteitsgroep Gezondheidsrisico Analyse en Toxicologie (GRAT). De studie werd afgesloten met twee stage-onderzoeken, waarvan de eerste is uitgevoerd bij TNO voeding te Zeist, divisie Toxicologie. Vervolgens werd een tweede stage uitgevoerd bij de capaciteitsgroep GRAT aan de Universiteit Maastricht. In maart 2000 behaalde ze haar doctoraal diploma. Vanaf april 2000 was Simone werkzaam als assistent in opleiding bij de capaciteitsgroep GRAT aan de Universiteit Maastricht waar het onderzoek dat beschreven is in dit proefschrift werd uitgevoerd. Naast het behalen van de certificaten 'Stralingshygiëne, deskundigheidsniveau $5 b$ ' en 'Proefdierkunde op grond van art.9 van de Wet op de Dierproeven' heeft zij verscheidene modules van de Postdoctorale Opleiding Toxicologie gevolgd om voor erkenning als toxicoloog in anmerking te komen. Vanaf januari 2005 zal Simone werkzaam zijn als post-doc bij de capaciteitsgroep GRAT aan de Universiteit Maastricht. 


\section{List of Publications}

\section{Articles}

1 Van Maanen, J.M., Albering, H.J., Van Breda, S.G., Curfs, D.M., Ambergen, A.W., Wolffenbuttel, B.H., Kleinjans, J.C. and Reeser, H.M. (1999) Nitrate in drinking water and risk of childhood diabetes in The Netherlands. Diabetes Cam, 22, 1750.

2 Van Maanen, J.M., Albering, H.J., de Kok, T.M., Van Breda, S.G., Curfs, D.M., Vermeer, I.T., Ambergen, A.W., Wolffenburtel, B.H., Kleinjans, J.C. and Reeser, H.M. (2000) Does the risk of childhood diabetes mellitus require revision of the guideline values for nitrate in drinking water? Emvinn Healtb Perspect, 108, 457-61.

3 Nia, A., Maas, L., Van Breda, S., Curfs, D., Kleinjans, J., Wouters, E. and Van Schooten, F.J. (2000) Applicability of induced sputum for molecular dosimetry of exposure to inhalatory carcinogens: $32 \mathrm{P}$-postlabeling of lipophilic DNA adducts in smokers and nonsmokers. Camer Epidemiol Biomarkers Proy, 9, 367-72.

Van Maanen, J.M., Albering, H.J., de Kok, T.M., Van Breda, S.G., Curfs, D.M., Vermeer, I.T., Ambergen, A.W., Wolffenbuttel, B.H., Kleinjans, J.C. and Reeser, H.M. (2000) Nitraat in drinkwater en risico voor type I diabetes in kinderen in Nederland. Nederlands Tijdscbrift voor Geneeskunde, 144, 1272-1276.

5 Van Delft, J.H., Van Agen, E., Van Breda, S.G., Herwijnen, M.H., Staal, Y.C. and Kleinjans, J.C. (2004) Discrimination of genotoxic from non-genotoxic carcinogens by gene expression profiling. Caninogenesis, 25, 1265-1276.

6 Van Breda, S.G., Van Agen, E., Van Sanden, S., Burzykowski, T., Kienhuis, A.S., Kleinjans, J.C. and Van Delft, J.H. (2004) Dose dependent effect of vegetables on gene expression in colon mucosa of C57BL6 female mice. Submitted.

7 Van Breda, S.G., Van Agen, E., Van Sanden, S., Burzykowski, T., Kleinjans, J.C. and Van Delft, J.H. (2004) Gene expression modulation by specific vegetables in colon mucosa of C57BL6 female mice. Submitted.

8 Van Breda, S.G., Van Agen, E., Van Sanden, S., Burzykowski, T., Kleinjans, J.C. and Van Delft, J.H. (2004) Gene expression modulation in lung cancer preventive pathways by vegetables in C57BL6 female mice. Submitted.

9 Van Breda, S.G., Van Agen, E., Engels, 1., Moonen, E.J., Kleinjans, J.C. and Van Delft, J. (2004) Altered vegetable intake affects pivotal carcinogenesis pathways in colon mucosa from adenoma patients and controls. In press, Caninogenesis.

10 Breikers, G., Van Breda, S.G., Bouwman, F.G., Van Herwijnen, M.H., Renes, J.W., Mariman, E.C., Kleinjans, J.C. and Van Delft, J.H. (2004) Identification of 
proteins differentially expressed in colon mucosa of C57BL6 female mice by altered vegetable intake. In preperation.

11 Van Delft, J.H., Van Agen, E., Van Breda, S.G., Herwijnen, M.H., Staal, Y.C. and Kleinjans, J.C. (2004) Comparison of supervised clustering methods to discriminate genotoxic from non-genotoxic carcinogens by gene expression profiling. Submitted.

\section{Abstracts}

$1 \quad$ Van Breda, S.G., Van Agen, E., Engels, L.G., Moonen, E.J., Kleinjans, J.C. and Van Delft, J.H. Identification of mechanisms at the genome level for protection against colon cancer by vegetables. Proceedings of the 31" Annual Meeting of the European Environmental Mutagen Society (EEMS), Ghent, Belgium, September 2001. Poster Award.

2 Van Breda, S.G., Van Agen, E., Engels, L.G., Moonen, E.J., Kleinjans, J.C. and Van Delft, J.H. Identification of mechanisms at the genome level for protection against colon cancer by vegetables. Proceedings of the scientific meeting of the Netherlands Society of Toxicology, Nijmegen, The Netherlands, November 2001.

3 Van Breda, S.G., Van Agen, E., Engels, L.G., Moonen, E.J., Kleinjans, J.C. and Van Delft, J.H. Modulation of gene expression by vegetables: identification of mechanisms for protection against colon cancer. Proceedings of the 1" Seminar of the European Graduate School, Maastricht, The Netherlands, November 2002. Oral presentation.

4 Van Breda, S.G., Van Agen, E., Engels, L.G., Moonen, E.J., Kleinjans, J.C. and Van Delft, J.H. Modulation of gene expression by vegetables: identification of mechanisms for protection against colon cancer. Proceedings of the scientific meeting of the Netherlands Society of Toxicology, De Bilt, The Netherlands, January 2003. Oral presentation.

$5 \quad$ Van Breda, S.G., Van Agen, E., Kienhuis, A.S., Kleinjans, J.C. and Van Delft, J.H. Dose-response study of vegetables on gene expression in colon mucosa of C57B16 female mice. 34 th Annual Meeting of the Environmental Mutagen Society (EMS), Miami Beach, USA, May 2003, Envionmental and Molecular Mutagenesis, 2003, 41: 210. EMS Student Achievement Award.

6 Van Breda, S.G., Van Agen, E., Engels, L.G., Moonen, E.J., Kleinjans, J.C. and Van Delft, J.H. Identification of mechanisms at the genome level for protection against colon cancer by vegetables. Proceedings of the $8^{\text {th }}$ International conference 
on Mechanisms of Antimutagenesis and anticarcinogenesis (ICMAA-VIII) Pisa, Italy, October 2003. Oral presentation.

7 Van Breda, S.G. Van Agen, E., Engels, L.G., Moonen, E.J., Kleinjans, J.C. and Van Delft, J.H. Identification of mechanisms at the genome level for protection against colon cancer by vegetables. Proceedings of the $2^{\text {nd }}$ Seminar of the European Graduate School, Maastricht, The Netherlands, October 2003. Oral presentation.

8 Van Breda, S.G. Van Agen, E., Engels, L.G., Moonen, E., J, Kleinjans, J.C. and Van Delft, J.H. Modulation of gene expression by vegetables in normal colorectal mucosa of sporadic colon adenoma patients and healthy controls. Proceedings of the Annual Mecting of the Netherlands Society of Gastroenterology (NVGE), Veldhoven, The Netherlands, March 2004. Oral presentation.

9 Van Delft, J.H., Van Agen, E., Van Breda. S.G., Van Henvijnen, M.H., Van Leeuwen, D.M., Staal, Y.C and Kleinjans, J.C. Toxicogenomics approaches in genetic toxicology. Proceedings of the scientific meeting of the Netherlands Society of Toxicology, Veldhoven, The Netherlands, June 2004.

10 Van Delft, J.H., Van Breda, S.G., Van Agen, E., Engels, L.G., Moonen, E.J., and Kleinjans, J.C. Identification of mechanisms at the genome level for protection against colon cancer by vegetables. NUGO, Wageningen, The Netherlands, September 2004.

11 Van Breda, S.G., Van Agen, E., Engels, I.G., Moonen, E.I., Kleinjans, J.C. and Van Delft, J.H. Altered vegetable intake affects pivotal carcinogenesis pathways in colon mucosa from adenoma patients and controls. Proceedings of the $34^{\text {r }}$ Annual Meeting of the European Environmental Mutagen Society (EEMS), Maastricht, The Netherlands, September 2004. Oral presentation.

12 Breikers, G., Van Breda, S.G., Bouwman, F.G., Van Herwijnen, M.H., Renes, J.W., Mariman, E.C., Kleinjans, J.C. and Van Delft, J.H. Identification of proteins differentially expressed in colon mucosa of C.57BL.6 female mice by altered vegetable intake. Proceedings of the 34" Annual Mecting of the European Environmental Mutagen Society (EEMS), Maastricht, The Netherlands, September 2004.

13 Van Breda, S.G., Van Agen, E., Engels, L.G., Moonen, F.J., Kleinjans, J.C. and Van Delft, J.H. Alteration in vegetable intake affects pivotal genetic pathways in colon carcinogenesis in sporadic colon adenoma patients and healthy controls. $12^{\text {th }}$ United European Gastroenterolgy Week (UEGWW). Prague, Czech Republic, September 2004. Young Investigators Travel Award; Oral presentation. 


\section{Abbreviations}

2-DE

$\mathrm{AC \#}$

$\mathrm{ACF}$

ACTB

$\triangle F P$

AICR

AL.DH1A1

AMACR

APC

ATF3

ATP

BCI.2

BNIP1

$\mathrm{CAH}-1$

CASP3

CASP4

CASP7

CCNA2

CCNG1

$\mathrm{CDH} 13$

$\mathrm{CDK}$

CDKN1A

cDNA

C.FOS

CHK1

C-MYB

COX-2

COXIV

CRC

CTNNB1

CTSB

CTSS

Cy 3

Cy5

CYP27B1

CYP2C19 two-dimensional polyacrylamide gel electrophoresis

genbank accession numbers

aberrant crypt foci

beta actin, cytoplasmic

alpha-fetoprotein

american institute for cancer research

aldehyde dehydrogenase family 1 , subfamily $\mathrm{A} 1$

methyl-Co $\mathrm{A}$ racemase alpha

adenomatous polyposis coli

activating transcription factor 3

adenosine triphosphate

breakdown cluster lymphoma

BCL.2/adenovirus E1B 19kDa-interacting protein 1

carbonic anhydrase 1

caspase 3 , apoptosis related cysteine protease

caspase 4 , apoptosis-related cysteine protease

caspase 7

cyclin $\mathrm{A} 2$

cyclin G1

cadherin $13 \mathrm{H}$-cadherin

cyclin-dependent kinase

cyclin-dependent kinase inhibitor $1 \mathrm{~A}$ (p21)

copy deoxyribonucleic acid

fos proto-oncogene

checkpoint kinase-1

c-myb proto-oncogene

cyclooxygenase- 2

cytochrome c oxidase subunit IV isoform 1

colorectal cancer

catenin gene

cathepsin B

cathepsin S

Cyanine 3

Cyanine 5

cytochrome P450 family 27 , subfamily B, polypeptide 1 .

cytochrome P450 family 2, subfamily C, polypeptide 19 


$\begin{array}{ll}\text { CYP2C9 } & \text { cytochrome P450 family 2, subfamily C, polypeptide 9 } \\ \text { CYP2D6 } & \text { cytochrome P450 family 2, subfamily D, polypeptide 6 } \\ \text { CYP3A4 } & \text { cytochrome P450 family 3, subfamily A, polypeptide 4 } \\ \text { DCC } & \text { deleted in colorectal cancer } \\ \text { DNA } & \text { deoxyribonucleic acid } \\ \text { DNES } & \text { diffuse neuroendocrine system } \\ \text { DRA } & \text { down-regulated in adenoma } \\ \text { DTT } & \text { dithiothreitol } \\ \text { e.g. } & \text { exempli gratia (for example) } \\ \text { EASE } & \text { expression analysis systematic explorer } \\ \text { EFHU1 } & \text { translation elongation factor EF-1 alpha-1 chain } \\ \text { ERBB } & \text { epidermal growth factor receptor } \\ \text { EST } & \text { expressed sequence tag } \\ \text { FAP } & \text { familial adenomatous polyposis } \\ \text { FCER1G } & \text { Fe receptor, lgE, high affinity 1, gamma polypeptide } \\ \text { FHIT } & \text { fragile histidine triad gene } \\ \text { G1P2 } & \text { interferon, alpha-inducible protein } \\ \text { GAPDH } & \text { glyceraldehyde-3-phosphate dehydrogenase } \\ \text { GLUL } & \text { glutamate-ammonia ligase (glutamine synthase) } \\ \text { GRP } & \text { gastrin-releasing peptide } \\ \text { GSR } & \text { glutathione reductase 1 } \\ \text { GSTA2 } & \text { glutathione S-transferase, alpha 2 (Yc2) } \\ \text { HCA } & \text { heterocyclic aromatic amines } \\ \text { HGF } & \text { hepatocyte growth factor; } \\ \text { HGF/MET } & \text { hypocyte growth factor/metastasis proto-oncogene } \\ \text { HIF1A } & \text { hypoxia inducible factor 1, alpha subunit } \\ \text { HMG-1 } & \text { high-mobility group protein 1 } \\ \text { hMLH } & \text { human mutL homolog } \\ \text { HNPCC } & \text { hereditary nonpolyposis colorectal cancer } \\ \text { HPGD } & \text { hydroxyprostaglandin dehydrogenase 15 NAD } \\ \text { HPLC-UV } & \text { high performance liquid chromatography-ultraviolet } \\ \text { hPMS } & \text { human postmeiotic segregation } \\ \text { HSPD1 } & \text { heat shock 60kDa protein 1 (chaperonin) } \\ \text { i.e. } & \text { id est (that is) } \\ \text { IGF-1 } & \text { insulin-like growth factor 1 } \\ \text { IGFBP3 } & \text { insulin-like growth factor binding protein 3 } \\ \text { IL18 } & \text { interleukin 18 } \\ \text { kDa } & \text { kilodalton } \\ \text { LDLR } & \text { low density lipoprotein receptor } \\ & \end{array}$




$\begin{array}{ll}\text { LOH } & \text { loss of heterozygosity } \\ \text { MALDI-MS } & \text { matrix-assisted laser desorption ionization mass spectroscopy } \\ \text { MDM2 } & \text { human mdm2-A } \\ \text { MLRN } & \text { smooth muscle isoform of myosin regulatory light chain 2 } \\ \text { MMR } & \text { mismatch repair } \\ \text { mRNA } & \text { messenger ribonucleic acid } \\ \text { MSI } & \text { microsatellite instability } \\ \text { MTHFR } & 5 \text {,10-methylenetetrahydrofolate reductase } \\ \text { MYBBP1a } & \text { MYB binding protein (P160) la } \\ \text { NCBI } & \text { national center for biotechnology information } \\ \text { NDF } & \text { new differentiation factor } \\ \text { NIA } & \text { national institute on aging } \\ \text { NSAIDs } & \text { non-steroidal anti-inflammatory drugs } \\ \text { NSCLC } & \text { non-small cell lung cancers } \\ \text { OAT } & \text { ornithine aminotransferase } \\ \text { ODC1 } & \text { ornithine decarboxylase 1 } \\ \text { OSCP } & \text { mitochondrial ATP synthase oligomycin sensitivity conferral precursor } \\ & \text { protein } \\ \text { PAP3 } & \text { pancreatitis-associated precursor protein 3 } \\ \text { PBS } & \text { phosphate buffered saline } \\ \text { PCNA } & \text { proliferating cell nuclear antigen gene } \\ \text { PKCB1 } & \text { protein kinase C beta 1 } \\ \text { PMP22 } & \text { peripheral myelin protein } \\ \text { PPAR } & \text { peroxisomal proliferators-activated receptor } \\ \text { PRDX1 } & \text { peroxiredoxin 1 } \\ \text { PTEN } & \text { phosphatase and tensin homolog } \\ \text { PTGS2 } & \text { prostaglandin-endoperoxide synthase 2 } \\ \text { RAR } & \text { retinoic acid receptor } \\ \text { RASSF1A } & \text { tumor suppressor RASSF1 isoform A } \\ \text { RB } & \text { retinoblastoma } \\ \text { Reg } & \text { regenerating gene } \\ \text { RNA } & \text { ribonucleic acid } \\ \text { RRM1 } & \text { ribonucleotide reductase M1 polypeptide } \\ \text { RT } & \text { room temperature } \\ \text { RT-PCR } & \text { reverse transcription polymerase chain reaction } \\ \text { RZPD } & \text { resource centre/primary database } \\ \text { SAGE } & \text { serial analysis of gene expression } \\ \text { SAT } & \text { spermidine/spermine N1-acetyl transferase 1 } \\ \text { SCD2 } & \text { stearoyl-Coenzyme A desaturase 2 } \\ & \end{array}$


$\mathrm{SCF} / \mathrm{KIT}$

SCIL

SDS

$\mathrm{SE}$

SEM

SEPP1

SLC26A3

SLC6A4

STAT1

SULT1A1

TCF/Lef

TGF-

TGFBR2

TGM2

TMSB10

TNFRSF6

TOP2A

WCRF

wt stem cell factor/tyrosine kinase receptor-oncogene KIT 0 small cell lung cancers

sodium dodecylsulfate

standard errot

standard error of the mean

selenoprotein $\mathrm{P}$, plasma 1

solute carrier family 26 , member 3

solute carrier family 6 (neurotransmitter transporter, serotonin), member 4 signal transducer and activator of transcription 1

sulfotransferase family $1 \mathrm{~A}$, phenol-preferring, member 1

T-cell factor/lymphoid enhancing factor

transforming growth factor beta

transforming growth factor beta receptor type II tumor suppressor gene transglutaminase 2, C polypeptide

thymosin, beta 10

tumor necrosis factor receptor superfamily, member 6

topoisomerase (DNA) II alpha $170 \mathrm{kDa}$

world cancer research fund

weight 
ISBN 90-6464-925-1 